\title{
RNAs Containing Carbocyclic Ribonucleotides (car-RNAs)
}

Masaaki Akabane-Nakata, Tyler Chickering, Joel M. Harp, $\uparrow$ Mark K. Schlegel, Shigeo Matsuda, Martin Egli, $\dagger$ and Muthiah Manoharan*

Alnylam Pharmaceuticals, 675 W Kendall Street, Cambridge, Massachusetts 02142, United States

$\dagger$ Department of Biochemistry, School of Medicine, Vanderbilt University, Nashville, Tennessee 37232, United States

*E-mail: mmanoharan@alnylam.com

SUPPLEMENTARY INFORMATION

\section{Table of Contents}

Synthetic procedures and characterization data for the new compounds ............................. S2

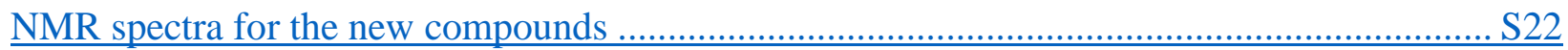

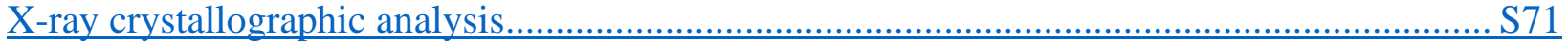

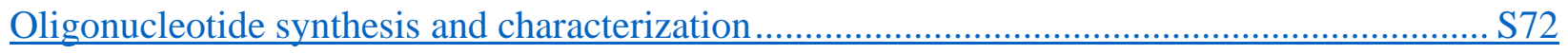

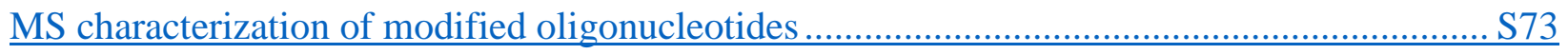

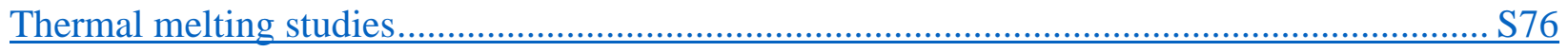

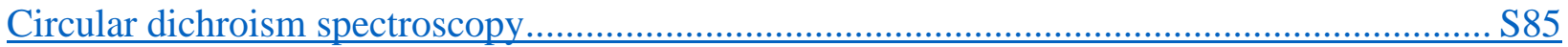

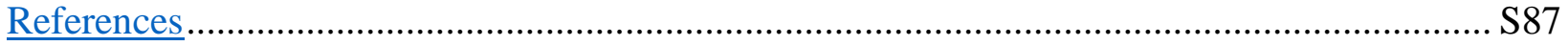




\section{Synthetic procedures and compound characterization}

\section{General conditions}

TLC was performed on Merck silica gel 60 plates coated with $F_{254}$. Compounds were visualized under UV light $(254 \mathrm{~nm}$ ) or after spraying with the $p$-anisaldehyde staining solution followed by heating. Flash column chromatography was performed using a Teledyne ISCO Combi Flash system with pre-packed RediSep Teledyne ISCO silica gel cartridges. All moisture-sensitive reactions were carried out under anhydrous conditions using dry glassware, anhydrous solvents, and argon atmosphere. The microwave reactions were performed using a Discover® SP microwave system (CEM corporation), heated in sealed glass tubes at $200 \mathrm{~W}$ with a 30 -sec premixing time. The reaction temperature was monitored with an internal infrared probe. All commercially available reagents and solvents were purchased from Sigma-Aldrich unless otherwise stated and were used as received. ESI-MS spectra were recorded on a Waters Qtof Premier instrument using the direct flow injection mode. ${ }^{1} \mathrm{H}$ NMR spectra were recorded at 400 or $500 \mathrm{MHz} .{ }^{13} \mathrm{C}$ NMR spectra were recorded at 101 or $126 \mathrm{MHz} .{ }^{31} \mathrm{P}$ NMR spectra were recorded at 162 or $202 \mathrm{MHz}$. Chemical shifts are given in ppm referenced to the solvent residual peak (DMSO$d_{6}-{ }^{1} \mathrm{H}: \delta$ at $2.50 \mathrm{ppm}$ and ${ }^{13} \mathrm{C} \delta$ at $39.5 \mathrm{ppm} ; \mathrm{CD}_{3} \mathrm{CN}-{ }^{1} \mathrm{H}: \delta$ at $1.94 \mathrm{ppm}$ and ${ }^{13} \mathrm{C} \delta$ at 1.32 and $118.3 \mathrm{ppm})$. Coupling constants are given in Hertz. Signal splitting patterns are described as singlet (s), doublet (d), triplet (t), septet (sept), broad signal (brs), or multiplet (m). 


\section{Synthesis of compound 8}

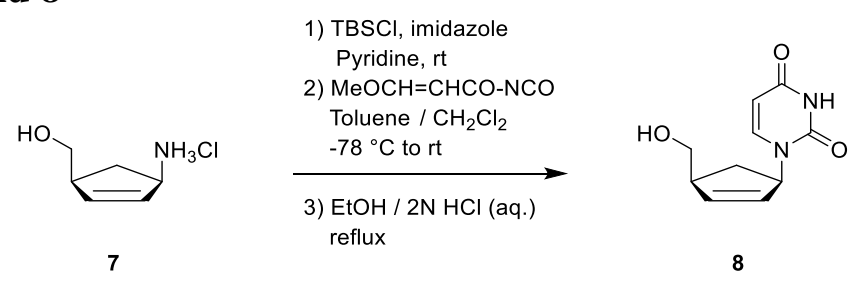

1) To a solution of $(1 S, 4 R)$-4-amino-2-cyclopentene-1-methanol hydrochloride (Biosynth Carbosynth: Product code FA32374) (7; $1.39 \mathrm{~g}$, $9.27 \mathrm{mmol})$ and imidazole (1.89 g, $27.8 \mathrm{mmol})$ in pyridine $(90 \mathrm{~mL})$ was added tert-butyldimethylsilyl chloride $(1.54 \mathrm{~g}, 10.2 \mathrm{mmol})$. After stirred at room temperature for $3 \mathrm{~h}$ the reaction mixture was concentrated under vacuum. The residue was dissolved in ethyl acetate and washed with saturated $\mathrm{NaHCO}_{3}$ (aq.) and brine. The organic layer was dried with $\mathrm{Na}_{2} \mathrm{SO}_{4}$ and concentrated under vacuum. The crude silyl-protected compound was dissolved in $\mathrm{CH}_{2} \mathrm{Cl}_{2}(30 \mathrm{~mL})$.

2) 3-methoxy acrylic acid $(2.84 \mathrm{~g}, 27.8 \mathrm{mmol})$ was dissolved in $\mathrm{CH}_{2} \mathrm{Cl}_{2} /$ pentane $(1: 1 ; 50 \mathrm{~mL})$. It was cooled to $0{ }^{\circ} \mathrm{C}$ in an ice bath, and oxalyl chloride $(11.8 \mathrm{~mL}, 139 \mathrm{mmol})$ was added to the solution dropwise. The solution was stirred at $0{ }^{\circ} \mathrm{C}$ for $2 \mathrm{~h}$. Solvent was removed, and the residual solid was kept under high vacuum for $30 \mathrm{~min}$. To the solution was added silver cyanate $(5.0 \mathrm{~g}$, $33.4 \mathrm{mmol}$ ) followed by refluxing for $2 \mathrm{~h}$. After cooling to room temperature, the liquid was carefully filtered through $0.2-\mu \mathrm{m}$ syringe filter and then cooled to $-78{ }^{\circ} \mathrm{C}$. The solution of the silylprotected compound in $\mathrm{CH}_{2} \mathrm{Cl}_{2}$ was added dropwise to this solution over $10 \mathrm{~min}$. The reaction mixture was then warmed to room temperature and stirred for $12 \mathrm{~h}$. The mixture was filtered and evaporated to a syrup.

3) The residue was dissolved in $2 \mathrm{~N} \mathrm{HCl}(18 \mathrm{~mL})$ and $\mathrm{EtOH}(54 \mathrm{~mL})$ and was refluxed at $90{ }^{\circ} \mathrm{C}$ for $3 \mathrm{~h}$. After excess solvent was removed, the crude residue was purified by column chromatography on silica gel $\left(0-10 \% \mathrm{MeOH}\right.$ in $\left.\mathrm{CH}_{2} \mathrm{Cl}_{2}\right)$ to obtain compound 8 as white solid $(1.12 \mathrm{~g}, 58 \%)$.

${ }^{1} \mathrm{H}$ NMR (400 MHz, DMSO- $\left.d_{6}\right) \delta 11.22(\mathrm{~s}, 1 \mathrm{H}), 7.46(\mathrm{~d}, J=8.0 \mathrm{~Hz}, 1 \mathrm{H}), 6.08$ (ddd, $J=2.1,2.1$ and $5.6 \mathrm{~Hz}, 1 \mathrm{H}), 5.67(\mathrm{ddd}, J=2.1,2.1$ and $5.6 \mathrm{~Hz}, 1 \mathrm{H}), 5.56(\mathrm{dd}, J=2.1$ and $8.0 \mathrm{~Hz}, 1 \mathrm{H}), 5.45-$ $5.51(\mathrm{~m}, 1 \mathrm{H}), 4.71(\mathrm{t}, J=5.2 \mathrm{~Hz}, 1 \mathrm{H}), 3.36-3.48(\mathrm{~m}, 2 \mathrm{H}), 2.75-2.82(\mathrm{~m}, 1 \mathrm{H}), 2.45-2.53(\mathrm{~m}, 1 \mathrm{H})$, 1.33 (ddd, $J=5.6,5.6$ and $13.9 \mathrm{~Hz}, 1 \mathrm{H}) .{ }^{13} \mathrm{C}$ NMR (101 MHz, DMSO- $\left.d_{6}\right) \delta 163.3,151.0,141.7$, 139.4, 129.5, 101.3, 63.5, 60.5, 47.3, 33.0. HRMS calc. for $\mathrm{C}_{10} \mathrm{H}_{12} \mathrm{~N}_{2} \mathrm{NaO}_{3}[\mathrm{M}+\mathrm{Na}]^{+} 231.0746$, found 231.0741 .

\section{Synthesis of compound 9}

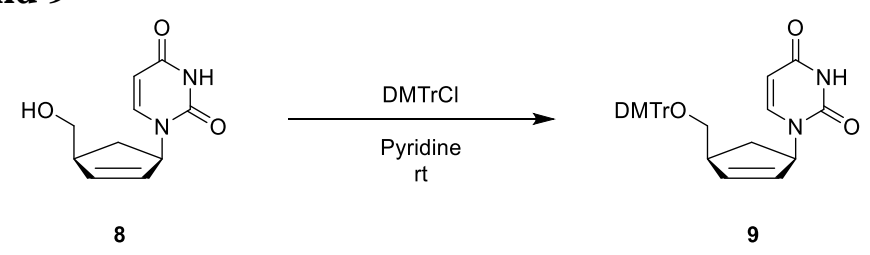

To a solution of compound $\mathbf{8}(1.0 \mathrm{~g}, 4.80 \mathrm{mmol})$ in pyridine $(50 \mathrm{~mL})$ was added 4,4-dimethoxytrityl chloride $(1.79 \mathrm{~g}, 5.28 \mathrm{mmol})$. The reaction mixture was stirred at $\mathrm{rt}$ for 3 hours before dry $\mathrm{MeOH}$ was added and the mixture was concentrated under vacuum. The residue was dissolved in ethyl acetate and the solution was washed with saturated $\mathrm{NaHCO}_{3}$ (aq.), water, brine, dried with $\mathrm{Na}_{2} \mathrm{SO}_{4}$ and concentrated under vacuum. The crude residue was purified by column chromatography on silica gel (25-50\% ethyl acetate in hexane) to obtain compound 9 as a white form $(2.21 \mathrm{~g}, 90 \%)$. ${ }^{1} \mathrm{H}$ NMR (400 MHz, DMSO-d6) $\delta 11.23(\mathrm{~s}, 1 \mathrm{H}), 7.19-7.36(\mathrm{~m}, 10 \mathrm{H}), 6.86-6.90(\mathrm{~m}, 4 \mathrm{H}), 6.16$ (ddd, $J=2.2,2.2$ and $5.6 \mathrm{~Hz}, 1 \mathrm{H}), 5.73$ (ddd, $J=2.2,2.2$ and $5.6 \mathrm{~Hz}, 1 \mathrm{H}), 5.41-5.45(\mathrm{~m}, 1 \mathrm{H}), 5.30$ 
(dd, $J=2.2$ and $7.9 \mathrm{~Hz}, 1 \mathrm{H}), 3.72(\mathrm{~s}, 6 \mathrm{H}), 3.11(\mathrm{dd}, J=5.6$ and $8.7 \mathrm{~Hz}, 1 \mathrm{H}), 2.92-2.99(\mathrm{~m}, 2 \mathrm{H})$, 1.21 (ddd, $J=5.6,5.6$ and $13.9 \mathrm{~Hz}, 1 \mathrm{H}) .{ }^{13} \mathrm{C}$ NMR $\left(101 \mathrm{MHz}, \mathrm{DMSO}-d_{6}\right) \delta 163.2,158.0,150.9$, 145.0, 141.3, 138.9, 135.6, 135.5, 129.7, 129.7, 129.7, 127.8, 127.6, 126.6, 113.2, 101.3, 85.4, 65.1, 60.5, 55.0, 44.8, 33.7. HRMS calc. for $\mathrm{C}_{31} \mathrm{H}_{30} \mathrm{~N}_{2} \mathrm{NaO}_{5}[\mathrm{M}+\mathrm{Na}]^{+}$533.2052, found 533.2065.

\section{Synthesis of compounds 10 and 11}

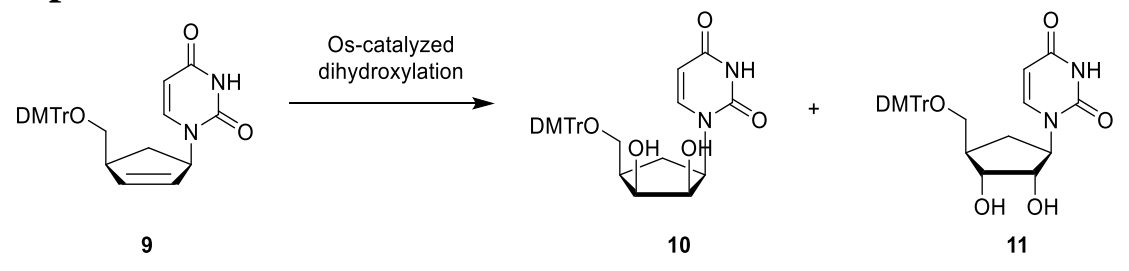

Table S1. Reaction conditions for Os-catalyzed dihydroxylation of olefin 9

\begin{tabular}{|c|c|c|c|c|c|c|}
\hline \multirow[b]{2}{*}{ entry } & \multirow[b]{2}{*}{ reagents } & \multirow[b]{2}{*}{ solvent } & \multirow[b]{2}{*}{ time } & \multicolumn{3}{|c|}{ yield (\%) } \\
\hline & & & & 10 & 11 & $10+11^{b}$ \\
\hline $1^{\mathrm{a}}$ & $\begin{array}{l}\text { AD-mix } \alpha(1.4 \mathrm{~g} / \mathrm{mmol}), \\
\mathrm{MeSO}_{2} \mathrm{NH}_{2}(1.0 \text { equiv. })\end{array}$ & $t-\mathrm{BuOH} / \mathrm{H}_{2} \mathrm{O}$ & 5 days & - & - & 47 \\
\hline 2 & $\begin{array}{l}\text { AD-mix } \alpha(4.0 \mathrm{~g} / \mathrm{mmol}), \\
\mathrm{MeSO}_{2} \mathrm{NH}_{2}(1.0 \text { equiv. })\end{array}$ & $t-\mathrm{BuOH} / \mathrm{H}_{2} \mathrm{O}$ & 3 days & 50 & 30 & 6 \\
\hline 3 & $\begin{array}{l}\text { AD-mix } \beta(4.0 \mathrm{~g} / \mathrm{mmol}) \\
\mathrm{MeSO}_{2} \mathrm{NH}_{2}(1.0 \text { equiv. })\end{array}$ & $t-\mathrm{BuOH} / \mathrm{H}_{2} \mathrm{O}$ & 2 days & 49 & 27 & 5 \\
\hline 4 & $\begin{array}{c}\mathrm{OsO}_{4}(3 \mathrm{~mol} \%) \\
\mathrm{NMO}(2.5 \text { equiv. })\end{array}$ & acetone $/ \mathrm{H}_{2} \mathrm{O}$ & $12 \mathrm{~h}$ & 36 & 39 & $<1$ \\
\hline
\end{tabular}

${ }^{a}$ See reference ${ }^{1}$

${ }^{\mathrm{b}}$ Diastereomeric ratio of $\mathbf{1 0}$ and $\mathbf{1 1}$ was not determined.

Entry 1: 9 (300 mg, $0.588 \mathrm{mmol})$ was dissolved in tert-butyl alcohol $t$ - $\mathrm{BuOH} / \mathrm{H}_{2} \mathrm{O}(2: 1,30 \mathrm{~mL})$. To the solution was added AD-mix $\alpha\left(1.4 \mathrm{~g}\right.$ per mmol) and methanesulfonamide $\left(\mathrm{MeSO}_{2} \mathrm{NH}_{2}\right.$; $55.9 \mathrm{mg}, 0.588 \mathrm{mmol}$ ) at $0{ }^{\circ} \mathrm{C}$. The reaction mixture was stirred at room temperature for 5 days. Even though the reaction was not completed, quenched by addition of saturated $\mathrm{NaHSO}_{3}$ (aq.). After diluted with ethyl acetate, the aqueous layer was extracted with ethyl acetate. The combined organic layer was dried with $\mathrm{Na}_{2} \mathrm{SO}_{4}$ and concentrated under vacuum. The crude residue was purified by column chromatography on silica gel (65-100\% ethyl acetate in hexane) to obtain a mixture of $\mathbf{1 0}$ and $\mathbf{1 1}$ as a white form $(150 \mathrm{mg}, 47 \%)$ and to recover $\mathbf{9}(85.1 \mathrm{mg}, 28 \%)$.

Entry 2: $9(1 \mathrm{~g}, 1.96 \mathrm{mmol})$ was dissolved in $t-\mathrm{BuOH} / \mathrm{H}_{2} \mathrm{O}(2: 1 ; 21 \mathrm{~mL})$ and AD-mix $\alpha(4.0 \mathrm{~g}$ per mmol) and methanesulfonamide $(186 \mathrm{mg}, 1.96 \mathrm{mmol})$ were added. The reaction mixture was stirred at $30{ }^{\circ} \mathrm{C}$ for 3 days. The reaction was quenched by the addition of saturated $\mathrm{Na}_{2} \mathrm{~S}_{2} \mathrm{O}_{3}$ (aq.) and the mixture was stirred for 30 min at room temperature. After diluted with $\mathrm{CH}_{2} \mathrm{Cl}_{2}$, the aqueous was extracted with $\mathrm{CH}_{2} \mathrm{Cl}_{2}$. The combined organic layer was dried with $\mathrm{Na}_{2} \mathrm{SO}_{4}$ and concentrated under vacuum. The crude residue was purified by column chromatography on silica gel (65-100\% ethyl acetate in hexane) to obtain compounds $\mathbf{1 0}$ as a white form (529 $\mathrm{mg}, 50 \%)$ and $\mathbf{1 1}$ as a white form (321 $\mathrm{mg}, 30 \%)$ and a mixture of the isomers $(68.9 \mathrm{mg}, 6 \%)$.

Entry 3: 9 (1 g, $1.96 \mathrm{mmol})$ was dissolved in $t-\mathrm{BuOH}_{2} \mathrm{H}_{2} \mathrm{O}(2: 1 ; 21 \mathrm{~mL})$ and AD-mix $\beta(4.0 \mathrm{~g}$ per $\mathrm{mmol})$ and methanesulfonamide $(186 \mathrm{mg}, 1.96 \mathrm{mmol})$ were added. The reaction mixture was stirred at $30{ }^{\circ} \mathrm{C}$ for 2 days. The reaction was quenched by the addition of saturated $\mathrm{Na}_{2} \mathrm{~S}_{2} \mathrm{O}_{3}$ (aq.) 
and the mixture was stirred for $30 \mathrm{~min}$ at room temperature. After diluted with $\mathrm{CH}_{2} \mathrm{Cl}_{2}$, the aqueous was extracted with $\mathrm{CH}_{2} \mathrm{Cl}_{2}$. The combined organic layer was dried with $\mathrm{Na}_{2} \mathrm{SO}_{4}$ and concentrated under vacuum. The crude residue was purified by column chromatography on silica gel (75-100\% ethyl acetate in hexane) to obtain compounds $\mathbf{1 0}$ as a white form (529 $\mathrm{mg}, 49 \%)$ and $\mathbf{1 1}$ as a white form $(290 \mathrm{mg}, 27 \%)$ and a mixture of the isomers $(56.1 \mathrm{mg}, 5 \%)$.

Entry 4: $9(850 \mathrm{mg}, 1.66 \mathrm{mmol})$ was dissolved in acetone $/ \mathrm{H}_{2} \mathrm{O}(5: 1 ; 12 \mathrm{~mL})$ and to the solution was added osmium tetroxide ( $4 \mathrm{wt} \% \mathrm{OsO}_{4}$ in $\mathrm{H}_{2} \mathrm{O}$ ) (3 mol\%) and $N$-methylmorpholine- $N$-oxide (NMO) $(486 \mathrm{mg}, 4.16 \mathrm{mmol})$. The mixture was stirred at room temperature for 12 hours. The reaction was quenched with a saturated $\mathrm{Na}_{2} \mathrm{~S}_{2} \mathrm{O}_{3}$ (aq.), and stirred for 30 min. The reaction mixture was diluted with ethyl acetate and the aqueous layer was extracted with ethyl acetate. The combined organic layers were dried $\left(\mathrm{Na}_{2} \mathrm{SO}_{4}\right)$ and concentrated under vacuum. The crude residue was purified by column chromatography on silica gel (65-100\% ethyl acetate in hexane) to obtain compounds $\mathbf{1 0}$ as a white form (325 mg, 36\%) and $\mathbf{1 1}$ as a white form (353 mg, 39\%) and a mixture of the isomers $(4.1 \mathrm{mg})$.

Compound 10: ${ }^{1} \mathrm{H}$ NMR (500 MHz, DMSO- $\left.d_{6}\right) \delta 11.11(\mathrm{~s}, 1 \mathrm{H}), 7.72(\mathrm{~d}, J=7.1 \mathrm{~Hz}, 1 \mathrm{H}), 7.37-$ $7.38(\mathrm{~m}, 2 \mathrm{H}), 7.18-7.30(\mathrm{~m}, 7 \mathrm{H}), 6.86-6.88(\mathrm{~m}, 4 \mathrm{H}), 5.53(\mathrm{dd}, J=2.5$ and $8.1 \mathrm{~Hz}, 1 \mathrm{H}), 5.13(\mathrm{~d}, J$ $=5.4 \mathrm{~Hz}, 1 \mathrm{H}), 4.94-5.02(\mathrm{~m}, 2 \mathrm{H}), 4.04-4.08(\mathrm{~m}, 1 \mathrm{H}), 3.96-3.98(\mathrm{~m}, 1 \mathrm{H}), 3.72(\mathrm{~s}, 6 \mathrm{H}), 3.25(\mathrm{dd}$, $J=6.3$ and $8.6 \mathrm{~Hz}, 1 \mathrm{H}), 2.94(\mathrm{dd}, J=7.2$ and $8.6 \mathrm{~Hz}, 1 \mathrm{H}), 2.06-2.14(\mathrm{~m}, 2 \mathrm{H}), 1.38-1.43(\mathrm{~m}, 1 \mathrm{H})$. ${ }^{13} \mathrm{C}$ NMR $\left(126 \mathrm{MHz}\right.$, DMSO- $\left.d_{6}\right) \delta 163.3,157.9,151.6,145.6,145.3,136.0,135.9,129.7,129.7$, 127.7, 127.69, 126.5, 113.1, 99.6, 85.1, 71.2, 71.0, 62.6, 55.0, 53.2, 32.8. HRMS calc. for $\mathrm{C}_{31} \mathrm{H}_{33} \mathrm{~N}_{2} \mathrm{O}_{7}[\mathrm{M}+\mathrm{H}]^{+}$545.2288, found 545.2297.

Compound 11: ${ }^{1} \mathrm{H}$ NMR $\left(500 \mathrm{MHz}, \mathrm{DMSO}-d_{6}\right) \delta 11.21(\mathrm{~s}, 1 \mathrm{H}), 7.58(\mathrm{~d}, J=8.1 \mathrm{~Hz}, 1 \mathrm{H}), 7.19$ $7.38(\mathrm{~m}, 9 \mathrm{H}), 6.88-6.89(\mathrm{~m}, 4 \mathrm{H}), 5.53(\mathrm{~d}, J=6.4 \mathrm{~Hz}, 1 \mathrm{H}), 4.89(\mathrm{~d}, J=6.1 \mathrm{~Hz}, 1 \mathrm{H}), 4.64(\mathrm{~d}, J=$ $4.7 \mathrm{~Hz}, 1 \mathrm{H}), 4.51-4.56(\mathrm{~m}, 1 \mathrm{H}), 3.94-3.98(\mathrm{~m}, 1 \mathrm{H}), 3.71-3.73(\mathrm{~m}, 7 \mathrm{H}), 3.09(\mathrm{dd}, J=5.6$ and 8.4 $\mathrm{Hz}, 1 \mathrm{H}), 2.95(\mathrm{dd}, J=6.8$ and $8.4 \mathrm{~Hz}, 1 \mathrm{H}), 2.02-2.07(\mathrm{~m}, 2 \mathrm{H}), 1.28-1.34(\mathrm{~m}, 1 \mathrm{H}) .{ }^{13} \mathrm{C}$ NMR $(126$ MHz, DMSO- $\left.d_{6}\right) \delta 163.2,158.0,151.2,145.1,143.0,135.8,129.7,127.8,127.7,126.6,113.1$, 101.1, 85.2, 72.9, 71.4, 64.7, 61.1, 55.0, 43.2, 28.3. HRMS calc. for $\mathrm{C}_{31} \mathrm{H}_{32} \mathrm{~N}_{2} \mathrm{NaO}_{7}[\mathrm{M}+\mathrm{Na}]^{+}$ 567.2107, found 567.2119. 
Scheme S1. Synthesis of aminotriol $\mathbf{1 2}^{2}$
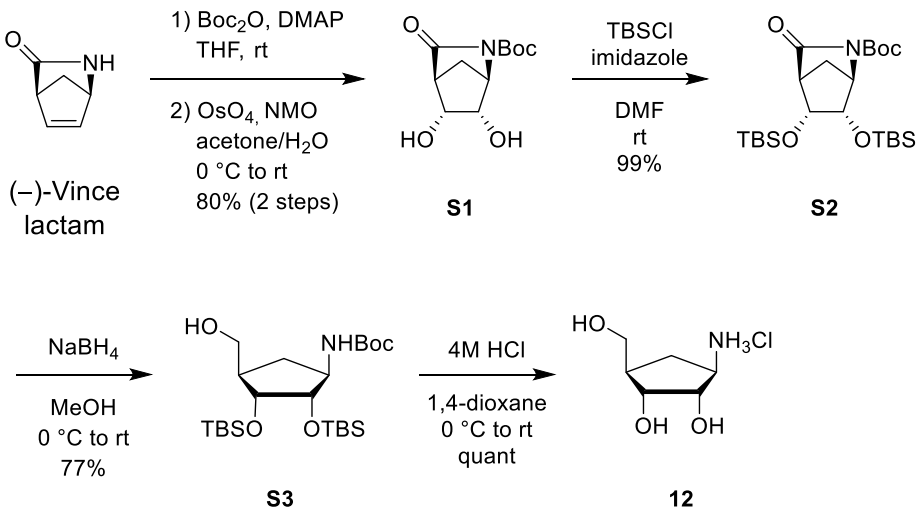

\section{Synthesis of compound $\mathrm{S1}^{3}$}

1) To a solution of (-)-Vince lactam (Biosynth Carbosynth: Product code FA18047) (10 g, 91.6 mmol) and DMAP (112 $\mathrm{mg}, 0.916 \mathrm{mmol})$ in THF $(500 \mathrm{~mL})$ was added dropwise di-tert-butyl dicarbonate $\left(\mathrm{Boc}_{2} \mathrm{O}\right)(21.1 \mathrm{~mL}, 91.6 \mathrm{mmol})$ at $0{ }^{\circ} \mathrm{C}$. The mixture was stirred at room temperature overnight. The reaction mixture was concentrated under vacuum. The residue was dissolved in ethyl acetate and washed with saturated aqueous $\mathrm{NaHCO}_{3}$, water and brine and dried $\left(\mathrm{Na}_{2} \mathrm{SO}_{4}\right)$ and concentrated under vacuum.

2) The crude residue was dissolved in acetone $(500 \mathrm{~mL})$ and $\mathrm{H}_{2} \mathrm{O}(50 \mathrm{~mL})$. To the solution was added 4-methylmorpholine $N$-oxide (NMO) $(21.5 \mathrm{~g}, 183 \mathrm{mmol})$ with stirring, followed by addition of aqueous $\mathrm{OsO}_{4}$ solution ( $4 \mathrm{wt} \%$ in $\mathrm{H}_{2} \mathrm{O} ; 1.17 \mathrm{~mL}, 0.2 \mathrm{~mol} \%$ ) at $0{ }^{\circ} \mathrm{C}$. The mixture was stirred at room temperature for $2 \mathrm{~h}$ and then concentrated under vacuum. The residue was purified by flash chromatography on silica gel (0-50\% ethyl acetate) to obtain compound $\mathbf{S 1}$ as a white form (17.1 $\mathrm{g}, 80 \%)$.

\section{Synthesis of compound $\mathrm{S2}^{2}$}

To a solution of $\mathbf{S 1}(19 \mathrm{~g}, 78.1 \mathrm{mmol})$ and imidazole $(26.6 \mathrm{~g}, 391 \mathrm{mmol})$ in DMF (200 mL) was added tert-butyldimethylsilyl chloride (TBSCl) $(35.3 \mathrm{mg}, 234 \mathrm{mmol})$ and the mixture was stirred at room temperature overnight. The reaction mixture was quenched with saturated $\mathrm{NaHCO}_{3}$ (aq.) and diluted with diethyl ether. The organic layer was washed with water, brine, dried $\left(\mathrm{Na}_{2} \mathrm{SO}_{4}\right)$ and concentrated under vacuum. The crude residue was purified by column chromatography on silica gel (0-10\% ethyl acetate in hexane) to obtain compound $\mathbf{S 2}$ as a white solid (36.6 g, 99\%).

\section{Synthesis of compound $\mathrm{S3}^{2}$}

To a solution of $\mathbf{S 2}(1 \mathrm{~g}, 2.12 \mathrm{mmol})$ in $\mathrm{MeOH}(20 \mathrm{~mL})$ was added $\mathrm{NaBH}_{4}(176 \mathrm{mg}, 4.66 \mathrm{mmol})$ at $0{ }^{\circ} \mathrm{C}$ and the mixture was stirred at $0{ }^{\circ} \mathrm{C}$ for $3 \mathrm{~h}$. The reaction mixture was concentrated under vacuum and the obtained residue was dissolved in $\mathrm{CH}_{2} \mathrm{Cl}_{2}$ and $1 \mathrm{~N} \mathrm{HCl}$ (aq.). The aqueous layer was extracted with $\mathrm{CH}_{2} \mathrm{Cl}_{2}$ and ethyl acetate. The combined organic layer was dried $\left(\mathrm{Na}_{2} \mathrm{SO}_{4}\right)$ and concentrated under vacuum. The crude residue was purified by column chromatography on silica gel (0-10\% ethyl acetate in hexane) to obtain compound $\mathbf{S 3}$ as a white solid (775 $\mathrm{mg}, 77 \%)$.

\section{Synthesis of compound $12^{2}$}

To a solution of $\mathrm{S} 3(1 \mathrm{~g}, 2.10 \mathrm{mmol})$ in 1,4-dioxane $(10 \mathrm{~mL})$ was added dropwise $\mathrm{HCl}(4.0 \mathrm{M}$ in dioxane, $5 \mathrm{~mL}, 20 \mathrm{mmol}$ ) and the mixture was stirred at room temperature overnight. The reaction mixture was filtrated to obtain a white precipitate. The white solid was washed with 1,4-dioxane to obtain compound $\mathbf{1 2}$ as a $\mathrm{HCl}$ salt (385 $\mathrm{mg}$, quant). 


\section{Synthesis of compound 13}
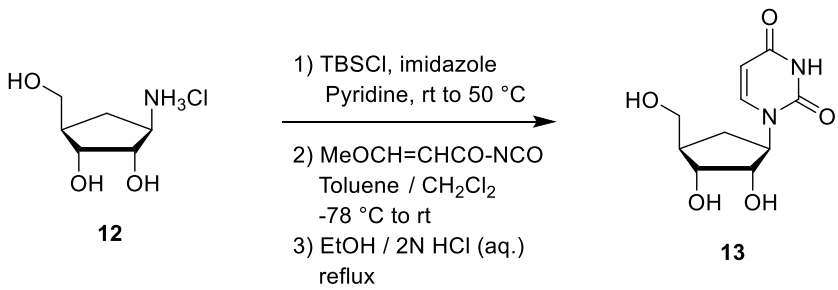

1) To a mixture of $(1 R, 2 S, 3 R, 4 R)$-2,3-dihydroxy-4-(hydroxymthyl)-1-aminocyclo-pentane hydrochloride (Millipore Sigma: Product ID ADV638390903) (12; $1 \mathrm{~g}, 5.45 \mathrm{mmol}$ ) and imidazole $(1.85 \mathrm{~g}, 27.2 \mathrm{mmol})$ in pyridine $(55 \mathrm{~mL})$ was added tert-butyldimethylsilyl chloride $(4.10 \mathrm{~g}, 27.2$ mmol). The mixture was stirred at room temperature for $48 \mathrm{~h}$, and then stirred at $40{ }^{\circ} \mathrm{C}$ for $24 \mathrm{~h}$ and at $50{ }^{\circ} \mathrm{C}$ for $24 \mathrm{~h}$. The reaction was cooled to room temperature and the solvent was removed under vacuum. The residue was dissolved in ethyl acetate and washed with saturated $\mathrm{NaHCO}_{3}$ (aq.) and brine. The organic layer was dried with $\mathrm{Na}_{2} \mathrm{SO}_{4}$ and concentrated under vacuum. The crude silyl-protected compound was dissolved in $\mathrm{CH}_{2} \mathrm{Cl}_{2}(30 \mathrm{~mL})$.

2) 3-methoxy acrylic acid $(1.53 \mathrm{~g}, 15.0 \mathrm{mmol})$ was dissolved in $\mathrm{CH}_{2} \mathrm{Cl}_{2} /$ pentane $(1: 1 ; 50 \mathrm{~mL})$. It was cooled to $0{ }^{\circ} \mathrm{C}$ in an ice bath, and oxalyl chloride $(6.35 \mathrm{~mL}, 75.0 \mathrm{mmol})$ was added to the solution dropwise. The solution was stirred at $0{ }^{\circ} \mathrm{C}$ for $2 \mathrm{~h}$. Solvent was removed, and the residual solid was kept under high vacuum for $30 \mathrm{~min}$. To the solution was added silver cyanate $(2.70 \mathrm{~g}$, $18.0 \mathrm{mmol}$ ) followed by refluxing for $2 \mathrm{~h}$. After cooling to room temperature, the liquid was carefully filtered through $0.2-\mu \mathrm{m}$ syringe filter and then cooled to $-78^{\circ} \mathrm{C}$. The solution of the silylprotected compound in $\mathrm{CH}_{2} \mathrm{Cl}_{2}$ was added dropwise to this solution over $10 \mathrm{~min}$. The reaction mixture was then warmed to room temperature and stirred for $18 \mathrm{~h}$. The reaction was quenched by adding $\mathrm{EtOH}$. The mixture was filtered and evaporated to a syrup.

3) The residue was dissolved in $2 \mathrm{~N} \mathrm{HCl}(18 \mathrm{~mL})$ and $\mathrm{EtOH}(54 \mathrm{~mL})$ and was refluxed at $90{ }^{\circ} \mathrm{C}$ for $3 \mathrm{~h}$. After excess solvent was removed, the crude residue was purified by column chromatography on silica gel $\left(0-15 \% \mathrm{MeOH}\right.$ in $\left.\mathrm{CH}_{2} \mathrm{Cl}_{2}\right)$ to provide compound $\mathbf{1 3}$ as white solid (770 mg, 58\%).

${ }^{1} \mathrm{H}$ NMR $\left(400 \mathrm{MHz}, \mathrm{DMSO}-d_{6}\right) \delta 11.18(\mathrm{~s}, 1 \mathrm{H}), 7.66(\mathrm{~d}, J=8.1 \mathrm{~Hz}, 1 \mathrm{H}), 5.58(\mathrm{~d}, J=8.1 \mathrm{~Hz}, 1 \mathrm{H})$, $4.84(\mathrm{~d}, J=5.6 \mathrm{~Hz}, 1 \mathrm{H}), 4.56-4.67(\mathrm{~m}, 3 \mathrm{H}), 3.96(\mathrm{ddd}, J=4.6,4.6$ and $4.6 \mathrm{~Hz}, 1 \mathrm{H}), 3.69-3.70(\mathrm{~m}$, $1 \mathrm{H}), 3.36-3.43(\mathrm{~m}, 2 \mathrm{H}), 1.86-2.02(\mathrm{~m}, 2 \mathrm{H}), 1.20-1.28(\mathrm{~m}, 1 \mathrm{H}) .{ }^{13} \mathrm{C}$ NMR (126 MHz, DMSO- $\left.d_{6}\right)$ $\delta$ 163.2, 151.4, 142.6, 101.2, 73.3, 71.5, 62.9, 60.0, 44.8, 27.8. HRMS calc. for $\mathrm{C}_{10} \mathrm{H}_{15} \mathrm{~N}_{2} \mathrm{O}_{5}[\mathrm{M}+$ $\mathrm{H}]^{+}$243.0981, found 243.0986.

\section{Synthesis of compound 11}

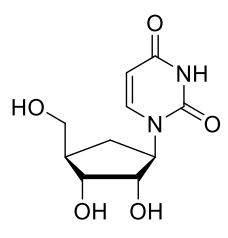

13

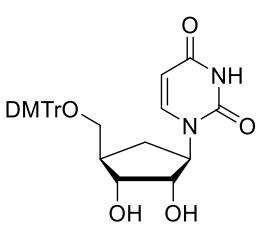

11

To a solution of compound $13(700 \mathrm{mg}, 2.89 \mathrm{mmol})$ in pyridine $(50 \mathrm{~mL})$ was added 4,4dimethoxytrityl chloride $(1.08 \mathrm{~g}, 3.18 \mathrm{mmol})$. The reaction mixture was stirred at room temperature for $5 \mathrm{~h}$ before dry $\mathrm{MeOH}$ was added and the mixture was concentrated under vacuum. The residue was dissolved in ethyl acetate and the solution was washed with saturated $\mathrm{NaHCO}_{3}$ (aq.), water, brine, dried with $\mathrm{Na}_{2} \mathrm{SO}_{4}$ and concentrated under vacuum. The crude residue was 
purified by column chromatography on silica gel (100\% ethyl acetate) to give the desired compound as a white form (1.58 g, quant).

${ }^{1} \mathrm{H}$ NMR $\left(500 \mathrm{MHz}, \mathrm{DMSO}-d_{6}\right) \delta 11.21(\mathrm{~s}, 1 \mathrm{H}), 7.58(\mathrm{~d}, J=8.1 \mathrm{~Hz}, 1 \mathrm{H}), 7.19-7.38(\mathrm{~m}, 9 \mathrm{H}), 6.88-$ $6.89(\mathrm{~m}, 4 \mathrm{H}), 5.53(\mathrm{~d}, J=6.4 \mathrm{~Hz}, 1 \mathrm{H}), 4.89(\mathrm{~d}, J=6.1 \mathrm{~Hz}, 1 \mathrm{H}), 4.64(\mathrm{~d}, J=4.7 \mathrm{~Hz}, 1 \mathrm{H}), 4.51-$ $4.56(\mathrm{~m}, 1 \mathrm{H}), 3.94-3.98(\mathrm{~m}, 1 \mathrm{H}), 3.71-3.73(\mathrm{~m}, 7 \mathrm{H}), 3.09(\mathrm{dd}, J=5.6$ and $8.4 \mathrm{~Hz}, 1 \mathrm{H}), 2.95(\mathrm{dd}$, $J=6.8$ and $8.4 \mathrm{~Hz}, 1 \mathrm{H}), 2.02-2.07(\mathrm{~m}, 2 \mathrm{H}), 1.28-1.34(\mathrm{~m}, 1 \mathrm{H}) .{ }^{13} \mathrm{C}$ NMR $\left(126 \mathrm{MHz}, \mathrm{DMSO}-d_{6}\right)$ $\delta 163.2,158.0,151.2,145.1,143.0,135.8,130.0,127.8,127.7,126.6,113.1,101.1,85.2,72.9$, 71.4, 64.7, 61.1, 55.0, 43.2, 28.3. HRMS calc. for $\mathrm{C}_{31} \mathrm{H}_{32} \mathrm{~N}_{2} \mathrm{NaO}_{7}[\mathrm{M}+\mathrm{Na}]^{+}$567.2107, found 567.2120 .

\section{Synthesis of compounds 14, 15 and 16}

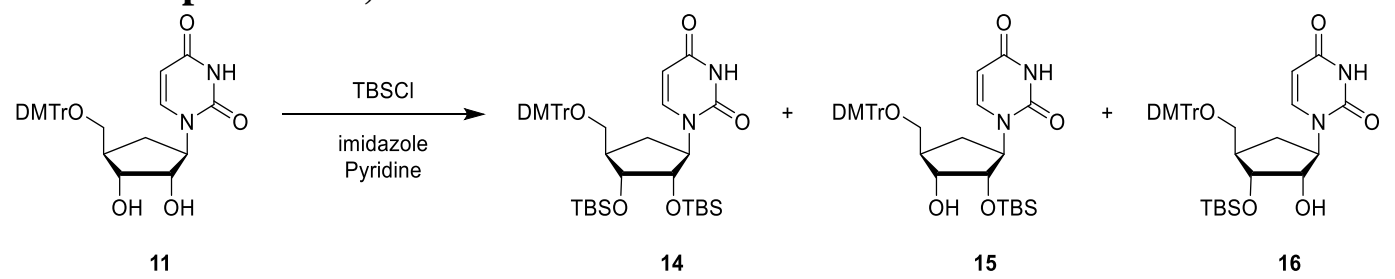

To a solution of compound $\mathbf{1 1}(1.0 \mathrm{~g}, 1.84 \mathrm{mmol})$ and imidazole $(375 \mathrm{mg}, 5.51 \mathrm{mmol})$ in pyridine (18.4 mL) was added tert-butyldimethylsilyl chloride $(304 \mathrm{mg}, 2.02 \mathrm{mmol})$ at $0{ }^{\circ} \mathrm{C}$. The mixture was stirred at $0{ }^{\circ} \mathrm{C}$ for $30 \mathrm{~min}$ and then further stirred at room temperature overnight. The reaction mixture was concentrated under vacuum. The residue was dissolved in ethyl acetate and washed with saturated $\mathrm{NaHCO}_{3}$ (aq.), water, brine, dried $\left(\mathrm{Na}_{2} \mathrm{SO}_{4}\right)$ and concentrated under vacuum. The crude residue was purified by column chromatography on silica gel (0-100\% ethyl acetate in hexane). Compound $\mathbf{1 4}$ was isolated as a white form (96.7 mg, 6.8\%), compound $\mathbf{1 5}$ was isolated as a white form (532 mg, 44\%), and compound $\mathbf{1 6}$ was isolated as a white form (415 mg, 34\%).

Compound 14: ${ }^{1} \mathrm{H}$ NMR (400 MHz, DMSO-d $) \delta 11.19(\mathrm{~s}, 1 \mathrm{H}), 7.53(\mathrm{~d}, J=8.0 \mathrm{~Hz}, 1 \mathrm{H}), 7.20$ $7.39(\mathrm{~m}, 9 \mathrm{H}), 6.86-6.88(\mathrm{~m}, 4 \mathrm{H}), 5.51(\mathrm{~d}, J=8.0 \mathrm{~Hz}, 1 \mathrm{H}), 4.52-4.59(\mathrm{~m}, 1 \mathrm{H}), 4.13(\mathrm{dd}, J=4.2$ and $9.2 \mathrm{~Hz}, 1 \mathrm{H}), 3.85(\mathrm{~d}, J=4.2 \mathrm{~Hz}, 1 \mathrm{H}), 3.72(\mathrm{~s}, 6 \mathrm{H}), 3.14(\mathrm{dd}, J=7.5$ and $9.2 \mathrm{~Hz}, 1 \mathrm{H}), 2.99$ (dd, $J=4.2$ and $9.2 \mathrm{~Hz}, 1 \mathrm{H}), 2.02-2.12(\mathrm{~m}, 2 \mathrm{H}), 1.39-1.51(\mathrm{~m}, 1 \mathrm{H}), 0.85(\mathrm{~s}, 9 \mathrm{H}), 0.77(\mathrm{~s}, 9 \mathrm{H}), 0.03(\mathrm{~s}$, $3 \mathrm{H}), 0.02$ (s, 3H), -0.07 (s, 3H), $-0.16(\mathrm{~s}, 3 \mathrm{H}) .{ }^{13} \mathrm{C}$ NMR (101 MHz, DMSO- $\left.d_{6}\right) \delta$ 163.0, 158.0, $150.9,144.9,144.4,135.5,129.7,127.7,127.6,126.6,113.1,101.0,85.4,74.0,73.9,64.3,61.8$, 55.0, 43.2, 25.8, 25.7, 25.5, 17.7, 17.5, -4.44, -4.56, -4.69, -5.29. HRMS calc. for $\mathrm{C}_{43} \mathrm{H}_{60} \mathrm{~N}_{2} \mathrm{NaO}_{7} \mathrm{Si}_{2}$ $[\mathrm{M}+\mathrm{Na}]^{+}$795.3837, found 795.3849.

Compound 15: ${ }^{1} \mathrm{H}$ NMR (400 MHz, DMSO- $\left.d_{6}\right) \delta 11.22(\mathrm{~s}, 1 \mathrm{H}), 7.61(\mathrm{~d}, J=8.0 \mathrm{~Hz}, 1 \mathrm{H}), 7.19-$ $7.39(\mathrm{~m}, 9 \mathrm{H}), 6.88-6.89(\mathrm{~m}, 4 \mathrm{H}), 5.54(\mathrm{~d}, J=8.0 \mathrm{~Hz}, 1 \mathrm{H}), 4.60-4.67(\mathrm{~m}, 1 \mathrm{H}), 4.38$ (d, $J=5.3 \mathrm{~Hz}$, $1 \mathrm{H}), 4.14(\mathrm{dd}, J=5.8$ and $8.4 \mathrm{~Hz}, 1 \mathrm{H}), 3.72-3.72(\mathrm{~m}, 7 \mathrm{H}), 3.11(\mathrm{dd}, J=6.8$ and $8.8 \mathrm{~Hz}, 1 \mathrm{H}), 2.96$ $(\mathrm{dd}, J=6.2$ and $8.8 \mathrm{~Hz}, 1 \mathrm{H}), 1.97-2.11(\mathrm{~m}, 2 \mathrm{H}), 1.41-1.49(\mathrm{~m}, 1 \mathrm{H}), 0.78(\mathrm{~s}, 9 \mathrm{H}),-0.02(\mathrm{~s}, 3 \mathrm{H})$, 0.08 (s, 3H). ${ }^{13} \mathrm{C}$ NMR $\left(126 \mathrm{MHz}, \mathrm{DMSO}-d_{6}\right) \delta 163.1,158.0,158.0,151.1,145.1,143.4,135.7$, 135.7, 129.7, 129.7, 127.8, 127.6, 126.6, 113.1, 101.1, 85.2, 74.9, 71.5, 64.5, 61.5, 55.0, 43.4, 26.8, 25.6, 17.8, -4.70, -5.20. HRMS calc. for $\mathrm{C}_{37} \mathrm{H}_{46} \mathrm{~N}_{2} \mathrm{NaO}_{7} \mathrm{Si}[\mathrm{M}+\mathrm{Na}]^{+}$681.2972, found 681.2964.

Compound 16: ${ }^{1} \mathrm{H}$ NMR (400 MHz, DMSO- $\left.d_{6}\right) \delta 11.20(\mathrm{~s}, 1 \mathrm{H}), 7.59$ (d, $\left.J=8.0 \mathrm{~Hz}, 1 \mathrm{H}\right), 7.19-$ $7.37(\mathrm{~m}, 9 \mathrm{H}), 6.86-6.88(\mathrm{~m}, 4 \mathrm{H}), 5.55(\mathrm{~d}, J=8.0 \mathrm{~Hz}, 1 \mathrm{H}), 4.72(\mathrm{~d}, J=6.5 \mathrm{~Hz}, 1 \mathrm{H}), 4.45-4.52(\mathrm{~m}$, $1 \mathrm{H}), 3.94-3.99(\mathrm{~m}, 1 \mathrm{H}), 3.83(\mathrm{dd}, J=3.4$ and $5.1 \mathrm{~Hz}, 1 \mathrm{H}), 3.72(\mathrm{~s}, 6 \mathrm{H}), 3.12(\mathrm{dd}, J=6.1$ and 9.2 $\mathrm{Hz}, 1 \mathrm{H}), 2.94(\mathrm{dd}, J=6.3$ and $9.2 \mathrm{~Hz}, 1 \mathrm{H}), 2.05-2.15(\mathrm{~m}, 2 \mathrm{H}), 1.29-1.36(\mathrm{~m}, 1 \mathrm{H}), 0.79(\mathrm{~s}, 9 \mathrm{H})$, $0.01(\mathrm{~s}, 3 \mathrm{H}),-0.04(\mathrm{~s}, 3 \mathrm{H}) .{ }^{13} \mathrm{C}$ NMR $\left(101 \mathrm{MHz}, \mathrm{DMSO}-d_{6}\right) \delta 163.2,158.0,151.2,145.0,143.3$, $135.7,135.7,129.6,129.6,127.8,127.6,126.6,113.1,101.0,85.4,73.7,72.5,64.5,61.3,55.0$, 
43.7, 28.0, 25.8, 18.0, -4.41, -5.05. HRMS calc. for $\mathrm{C}_{37} \mathrm{H}_{46} \mathrm{~N}_{2} \mathrm{NaO}_{7} \mathrm{Si}[\mathrm{M}+\mathrm{Na}]^{+}$681.2972, found 681.2981 .

\section{Synthesis of compound 1}
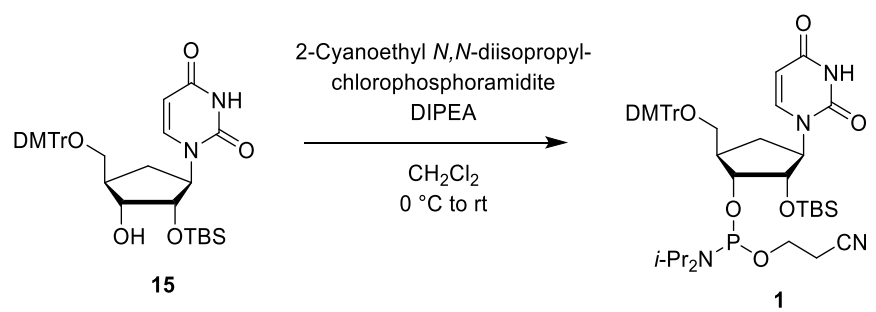

To a solution of compound 15 (1.50 g, $2.28 \mathrm{mmol})$ and DIPEA (1.19 mL, $6.83 \mathrm{mmol})$ in $\mathrm{CH}_{2} \mathrm{Cl}_{2}$ $(23 \mathrm{~mL})$ was added dropwise 2-cyanoethyl $N, N$-diisopropylchlorophosphoramidite $(0.609 \mathrm{~mL}$, $2.35 \mathrm{mmol}$ ) at $0{ }^{\circ} \mathrm{C}$. The mixture was stirred at room temperature for $1 \mathrm{~h}$. The reaction mixture was then cooled to $0^{\circ} \mathrm{C}$ and added dropwise 2-cyanoethyl $N, N$-diisopropylchlorophosphoramidite $(0.406 \mathrm{~mL}, 1.82 \mathrm{mmol})$ at $0{ }^{\circ} \mathrm{C}$. The mixture was stirred at room temperature for $3 \mathrm{~h}$ and quenched with saturated $\mathrm{NaHCO}_{3}$ (aq.). The organic layer was washed with saturated $\mathrm{NaHCO}_{3}$ (aq.), water, brine, dried $\left(\mathrm{Na}_{2} \mathrm{SO}_{4}\right)$ and concentrated under vacuum. The crude residue was purification by column chromatography on silica gel (40\% ethyl acetate in hexane) to obtain compound $\mathbf{1}$ as a white form $(1.64 \mathrm{~g}, 79 \%)$.

${ }^{1} \mathrm{H}$ NMR $\left(500 \mathrm{MHz}, \mathrm{CD}_{3} \mathrm{CN}\right) \delta 9.09(\mathrm{~s}, 1 \mathrm{H}), 7.45-7.48(\mathrm{~m}, 2 \mathrm{H}), 7.21-7.35(\mathrm{~m}, 8 \mathrm{H}), 6.86-6.89(\mathrm{~m}$, $4 \mathrm{H}), 5.51-5.54(\mathrm{~m}, 1 \mathrm{H}), 4.66-4.72(\mathrm{~m}, 1 \mathrm{H}), 4.28-4.33(\mathrm{~m}, 1 \mathrm{H}), 4.00-4.07(\mathrm{~m}, 1 \mathrm{H}), 3.88-3.94(\mathrm{~m}$, $1 \mathrm{H}), 3.80-3.86(\mathrm{~m}, 1 \mathrm{H}), 3.76(\mathrm{~s}, 6 \mathrm{H}), 3.59-3.66(\mathrm{~m}, 2 \mathrm{H}), 3.11-3.22(\mathrm{~m}, 2 \mathrm{H}), 2.46-2.71(\mathrm{~m}, 2 \mathrm{H})$, $2.32-2.38(\mathrm{~m}, 1 \mathrm{H}), 2.15-2.20(\mathrm{~m}, 1 \mathrm{H}), 1.62-1.68(\mathrm{~m}, 1 \mathrm{H}), 1.10-1.19(\mathrm{~m}, 12 \mathrm{H}), 0.82-0.84(\mathrm{~m}, 9 \mathrm{H})$, $0.03-0.05(\mathrm{~m}, 3 \mathrm{H}),-0.05(\mathrm{~s}, 3 \mathrm{H}) .{ }^{13} \mathrm{C} \mathrm{NMR}\left(101 \mathrm{MHz}, \mathrm{CD}_{3} \mathrm{CN}\right) \delta 164.0,159.7,152.1,152.1$, $146.4,146.3$, 144.8, 144.4, 137.1, 137.1, 137.0, 137.0, 131.2, 131.1, 129.1, 129.1, 128.9, 127.9, $119.7,119.5,114.1,102.4,87.2,87.2,77.1,77.0,76.3,76.3,75.9,75.9,75.7,75.5,65.5,65.3$, 63.2, 63.2, 60.1, 59.9, 58.9, 58.7, 56.0, 44.3, 44.2, 43.8, 43.7, 43.1, 43.0, 27.1, 26.7, 26.3, 26.3, 26.2, 25.3, 25.2, 25.2, 25.1, 25.0, 24.9, 21.3, 21.3, 21.1, 21.1, 18.7, 18.7, -3.79, -3.83, -4.57, -4.61. ${ }^{31} \mathrm{P}$ NMR $\left(162 \mathrm{MHz}, \mathrm{CD}_{3} \mathrm{CN}\right) \delta 149.96,147.67$. HRMS calc. for $\mathrm{C}_{46} \mathrm{H}_{64} \mathrm{~N}_{4} \mathrm{O}_{8} \mathrm{PSi}[\mathrm{M}+\mathrm{H}]^{+}$ 859.4231, found 859.4242 .

\section{Synthesis of compound 2}
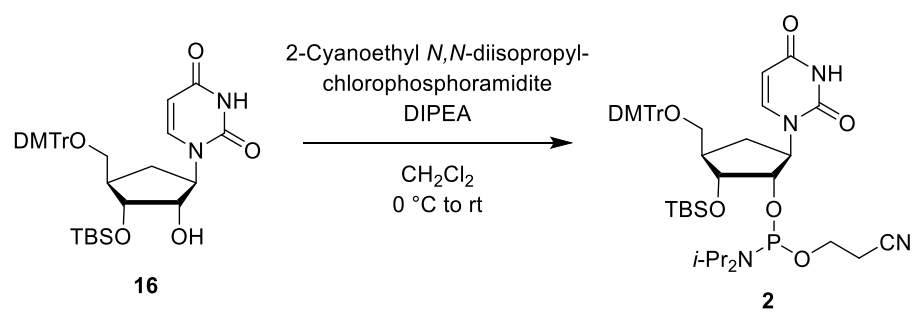

To a solution of compound $16(1.10 \mathrm{~g}, 1.67 \mathrm{mmol})$ and DIPEA $(0.872 \mathrm{~mL}, 5.01 \mathrm{mmol})$ in $\mathrm{CH}_{2} \mathrm{Cl}_{2}$ $(17 \mathrm{~mL})$ was added dropwise 2-cyanoethyl $N, N$-diisopropylchlorophosphoramidite $(0.447 \mathrm{~mL}$, $2.00 \mathrm{mmol}$ ) at $0{ }^{\circ} \mathrm{C}$. The mixture was stirred at $\mathrm{rt}$ for $1 \mathrm{~h}$. The reaction mixture was then cooled to $0{ }^{\circ} \mathrm{C}$ and added dropwise 2-cyanoethyl $N, N$-diisopropylchlorophosphoramidite $(0.298 \mathrm{~mL}, 1.34$ mmol) at $0{ }^{\circ} \mathrm{C}$. The mixture was stirred at $\mathrm{rt}$ for $3 \mathrm{~h}$ and quenched with saturated $\mathrm{NaHCO}_{3}$ (aq.) and washed with saturated $\mathrm{NaHCO}_{3}$ (aq.), water, brine, dried $\left(\mathrm{Na}_{2} \mathrm{SO}_{4}\right)$ and concentrated under 
vacuum. The crude residue was purification by column chromatography on silica gel (40\% ethyl acetate in hexane) to give compound 2 as a white form $(1.17 \mathrm{~g}, 78 \%)$.

${ }^{1} \mathrm{H}$ NMR $\left(400 \mathrm{MHz}, \mathrm{CD}_{3} \mathrm{CN}\right) \delta 9.02(\mathrm{~s}, 1 \mathrm{H}), 7.43-7.46(\mathrm{~m}, 2 \mathrm{H}), 7.21-7.34(\mathrm{~m}, 8 \mathrm{H}), 6.86-6.90(\mathrm{~m}$, $4 \mathrm{H}), 5.58(\mathrm{~d}, J=8.0 \mathrm{~Hz}, 0.5 \mathrm{H}), 5.49(\mathrm{~d}, J=8.0 \mathrm{~Hz}, 0.5 \mathrm{H}), 4.74-4.87(\mathrm{~m}, 1 \mathrm{H}), 3.99-4.35(\mathrm{~m}, 2 \mathrm{H})$, 3.49-3.77 (m, 10H), 3.07-3.17 (m, 2H), 2.49-2.55 (m, 2H), 2.17-2.25 (m, 2H), 1.14-1.60 (m, 1H), 1.03-1.13 (m, 12H), 0.88-0.89 (m, 9H), $0.12(\mathrm{~s}, 1.5 \mathrm{H}), 0.07(\mathrm{~s}, 1.5 \mathrm{H}), 0.07(\mathrm{~s}, 1.5 \mathrm{H}), 0.05(\mathrm{~s}$, $1.5 \mathrm{H}) .{ }^{13} \mathrm{C}$ NMR $\left(101 \mathrm{MHz}, \mathrm{CD}_{3} \mathrm{CN}\right) \delta 164.2,164.0,159.7,159.7,152.2,152.1,146.4,146.3$, 144.9 , 144.1, 137.1, 137.1, 131.2, 131.1, 131.1, 129.1, 129.0, 128.9, 128.9, 127.9, 127.9, 119.5, $114.2,114.1,114.1,102.4,102.2,87.4,87.1,77.9,77.7,75.8,75.7,74.8,74.8,74.6,74.6,65.7$, 65.6, 62.6, 61.7, 61.7, 59.6, 59.4, 59.2, 59.0, 56.0, 56.0, 45.1, 45.0, 44.0, 43.9, 43.9, 43.8, 27.7, 27.3, 26.4, 25.3, 25.2, 25.0, 25.0, 25.0, 24.9, 24.8, 21.1, 21.0, 21.0, 20.9, 18.8, 18.8, -3.92, -3.94, $-4.01,-4.03,-4.15,-4.23,-4.24 .{ }^{31} \mathrm{P}$ NMR $\left(202 \mathrm{MHz}, \mathrm{CD}_{3} \mathrm{CN}\right) \delta 150.97,149.00$. HRMS calc. for $\mathrm{C}_{46} \mathrm{H}_{64} \mathrm{~N}_{4} \mathrm{O}_{8} \mathrm{PSi}[\mathrm{M}+\mathrm{H}]^{+} 859.4231$, found 859.4235.

\section{Synthesis of compound 17}
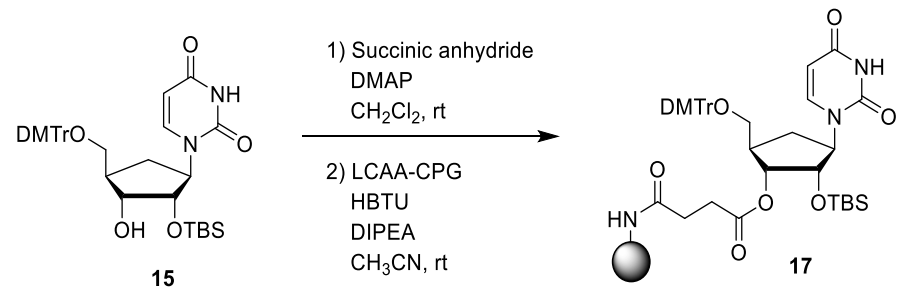

To a solution of compound 15 (300 mg, $0.455 \mathrm{mmol})$ in $\mathrm{CH}_{2} \mathrm{Cl}_{2}(4 \mathrm{~mL})$ were added succinic anhydride $(91.1 \mathrm{mg}, 0.911 \mathrm{mmol})$ and DMAP $(111 \mathrm{mg}, 0.911 \mathrm{mmol})$, and the mixture was stirred at room temperature for $3 \mathrm{~h}$. The reaction mixture was concentrated undervacuum. The residue was filtered through a short silica gel pad using $10 \% \mathrm{MeOH}$ in $\mathrm{CH}_{2} \mathrm{Cl}_{2}$ and the filtrate was concentrated under vacuum. The obtained succinate was dissolved in acetonitrile $(10 \mathrm{~mL})$ and the solution was added to CPG functionalized with long chain amino alkyl (LCAA) (pore size $500 \AA$ $\mathrm{NH}_{2}$, loading of $\left.171 \mu \mathrm{mol} / \mathrm{g}, 3 \mathrm{~g}\right)$, DIPEA $(0.317 \mathrm{~mL}, 1.82 \mathrm{mmol})$ and HBTU (190 mg, 0.501 $\mathrm{mmol}$ ). The mixture was agitated on a wrist-action shaker at room temperature overnight and filtered and then washed with acetonitrile, $\mathrm{MeOH}$, acetonitrile, and diethyl ether. The obtained CPG was added to a mixture of CapA solution and CapB solution $(1: 1, \mathrm{v} / \mathrm{v})$ and the mixture was agitated on a wrist-action shaker at room temperature overnight. The reaction mixture was filtered and washed with $10 \% \mathrm{H}_{2} \mathrm{O} / \mathrm{THF}, \mathrm{MeOH}, 10 \% \mathrm{H}_{2} \mathrm{O} / \mathrm{THF}, \mathrm{MeOH}$, acetonitrile, and ethyl ether to obtain the CPG support 17 (2.89 g, $100.78 \mu \mathrm{mol} / \mathrm{g})$.

\section{Synthesis of compound 18}

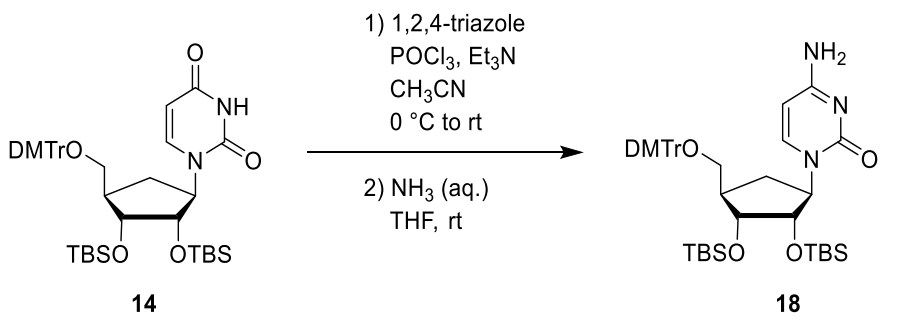

1) Phosphorus oxychloride $(0.362 \mathrm{~mL}, 3.88 \mathrm{mmol})$ was added dropwise to a solution of 1,2,4triazole $(2.14 \mathrm{~g}, 31.0 \mathrm{mmol})$ and $\mathrm{Et}_{3} \mathrm{~N}(4.33 \mathrm{~mL}, 31.0 \mathrm{mmol})$ in $\mathrm{CH}_{3} \mathrm{CN}(10 \mathrm{~mL})$ at $0{ }^{\circ} \mathrm{C}$. After stirring for $30 \mathrm{~min}$, a solution of compound $14(1.0 \mathrm{~g}, 1.29 \mathrm{mmol})$ in $\mathrm{CH}_{3} \mathrm{CN}(3 \mathrm{~mL})$ was added dropwise to the reaction mixture at $0^{\circ} \mathrm{C}$. After stirring for $10 \mathrm{~min}$, the reaction was stirred at room 
temperature for $3 \mathrm{~h}$. The reaction was quenched with saturated $\mathrm{NaHCO}_{3}$ (aq.) and diluted with ethyl acetate. The organic layer was washed with water, brine, dried $\left(\mathrm{Na}_{2} \mathrm{SO}_{4}\right)$ and concentrated under vacuum.

2) The crude residue was dissolved in THF (12 mL) and ammonium hydroxyde (12 mL) was added. The reaction mixture was stirred at room temperature overnight and diluted with ethyl acetate. The solution was sequentially washed with water, brine, dried $\left(\mathrm{Na}_{2} \mathrm{SO}_{4}\right)$ and concentrated under vacuum. The residue was purified by flash chromatography on silica gel (100\% ethyl acetate) to give compound $\mathbf{1 8}$ as a white form $(883 \mathrm{mg}, 89 \%)$.

${ }^{1} \mathrm{H}$ NMR (400 MHz, DMSO-d $)$ d 7.38-7.41 (m, 3H), 7.20-7.31 (m, 7H), 6.86-6.94 (m, 6H), 5.60 $(\mathrm{d}, J=7.3 \mathrm{~Hz}, 1 \mathrm{H}), 4.38-4.45(\mathrm{~m}, 1 \mathrm{H}), 4.25(\mathrm{dd}, J=4.4$ and $8.6 \mathrm{~Hz}, 1 \mathrm{H}), 3.89-3.90(\mathrm{~m}, 1 \mathrm{H}), 3.72$ $(\mathrm{s}, 6 \mathrm{H}), 3.16(\mathrm{dd}, J=7.7$ and $9.1 \mathrm{~Hz}, 1 \mathrm{H}), 2.97(\mathrm{dd}, J=4.7$ and $9.1 \mathrm{~Hz}, 1 \mathrm{H}), 2.00-2.13(\mathrm{~m}, 2 \mathrm{H})$, 1.48-1.56 (m, 1H), $0.84(\mathrm{~s}, 9 \mathrm{H}), 0.76(\mathrm{~s}, 9 \mathrm{H}), 0.02(\mathrm{~s}, 6 \mathrm{H}),-0.10(\mathrm{~s}, 3 \mathrm{H}),-0.20(\mathrm{~s}, 3 \mathrm{H}) .{ }^{13} \mathrm{C}$ NMR $\left(101 \mathrm{MHz}, \mathrm{DMSO}-d_{6}\right) \delta 165.2,158.0,155.6,145.2,145.0,135.6,129.7,127.7,127.6,126.6,113.1$, 93.2, 85.4, 74.3, 74.0, 64.5, 63.7, 55.0, 43.3, 26.5, 25.7, 25.7, 17.7, 17.5, -4.53, -4.59, -4.60, -5.11. HRMS calc. for $\mathrm{C}_{43} \mathrm{H}_{62} \mathrm{~N}_{3} \mathrm{O}_{6} \mathrm{Si}_{2}[\mathrm{M}+\mathrm{H}]^{+} 772.4177$, found 772.4183 .

\section{Synthesis of compound 19}

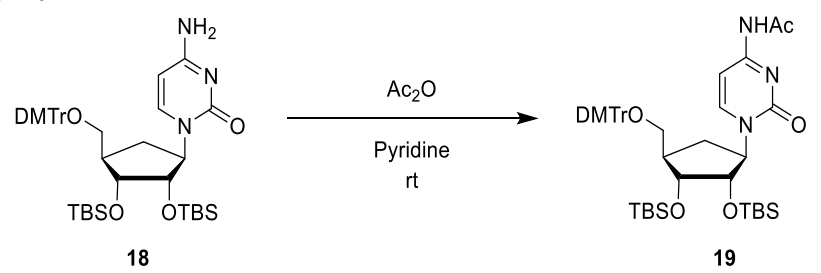

To a solution of compound $\mathbf{1 8}(750 \mathrm{mg}, 0.971 \mathrm{mmol})$ in pyridine $(10 \mathrm{~mL})$ was added acetic anhydride (149 mg, $1.46 \mathrm{mmol})$ and the mixture was stirred at room temperature for $2 \mathrm{~h}$. The reaction mixture was quenched with saturated $\mathrm{NaHCO}_{3}$ (aq.) and diluted with ethyl acetate. The organic layer was washed with water, brine, dried $\left(\mathrm{Na}_{2} \mathrm{SO}_{4}\right)$ and concentrated under vacuum. The crude residue was purified by column chromatography on silica gel (50-75\% ethyl acetate in hexane) to give compound $\mathbf{1 9}$ as a white form (766 mg, 97\%).

${ }^{1} \mathrm{H}$ NMR (400 MHz, DMSO-d6) $\delta 10.79(\mathrm{~s}, 1 \mathrm{H}), 7.96(\mathrm{~d}, J=7.4 \mathrm{~Hz}, 1 \mathrm{H}), 7.20-7.41(\mathrm{~m}, 9 \mathrm{H}), 7.11$ $(\mathrm{d}, J=7.4 \mathrm{~Hz}, 1 \mathrm{H}), 6.86-6.89(\mathrm{~m}, 6 \mathrm{H}), 4.54-4.59(\mathrm{~m}, 1 \mathrm{H}), 4.32(\mathrm{dd}, J=4.1$ and $8.9 \mathrm{~Hz}, 1 \mathrm{H}), 3.89$ $(\mathrm{d}, J=4.1 \mathrm{~Hz}, 1 \mathrm{H}), 3.72(\mathrm{~s}, 6 \mathrm{H}), 3.19(\mathrm{dd}, J=7.8$ and $9.1 \mathrm{~Hz}, 1 \mathrm{H}), 3.01(\mathrm{dd}, J=4.0$ and $9.1 \mathrm{~Hz}$, $1 \mathrm{H}), 2.06-2.11(\mathrm{~m}, 5 \mathrm{H}), 1.61-1.68(\mathrm{~m}, 1 \mathrm{H}), 0.85(\mathrm{~s}, 9 \mathrm{H}), 0.73(\mathrm{~s}, 9 \mathrm{H}), 0.03(\mathrm{~s}, 3 \mathrm{H}), 0.03(\mathrm{~s}, 3 \mathrm{H})$, $-0.11(\mathrm{~s}, 3 \mathrm{H}),-0.25$ (s, 3H). ${ }^{13} \mathrm{C}$ NMR $\left(101 \mathrm{MHz}, \mathrm{DMSO}-d_{6}\right) \delta 170.9,161.7,158.0,155.1,149.6$, 145.0, 135.6, 129.7, 127.7, 127.6, 126.6, 113.1, 95.0, 85.4, 74.2, 73.7, 64.7, 64.3, 55.0, 43.4, 25.9, $25.7,25.6,24.3,17.7,17.5,-4.55,-4.57,-4.63,-5.28$. HRMS calc. for $\mathrm{C}_{45} \mathrm{H}_{64} \mathrm{~N}_{3} \mathrm{O}_{7} \mathrm{Si}_{2}[\mathrm{M}+\mathrm{H}]^{+}$ 814.4283 , found 814.4286 . 


\section{Synthesis of compound 20}

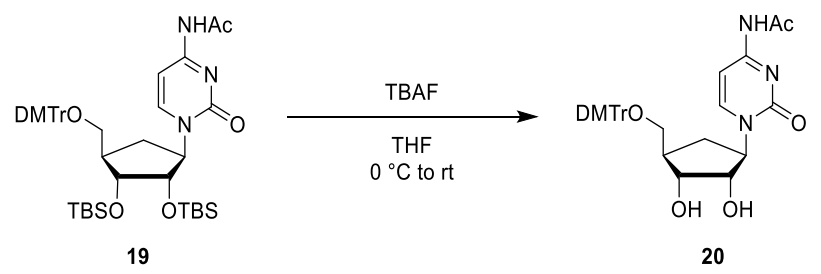

To a solution of compound $19(650 \mathrm{mg}, 0.798 \mathrm{mmol})$ in THF $(8 \mathrm{~mL})$ was added dropwise tetra- $n$ butylammonium fluoride (1M in THF; $2.40 \mathrm{~mL}, 2.40 \mathrm{mmol})$ at $0{ }^{\circ} \mathrm{C}$ and the mixture was stirred at $\mathrm{rt}$ for $12 \mathrm{~h}$. After the solvent was removed under vacuum the crude residue was purified by column chromatography on silica gel $\left(0-15 \% \mathrm{MeOH}\right.$ in $\left.\mathrm{CH}_{2} \mathrm{Cl}_{2}\right)$ to give compound 20 as a white solid (496 mg, quant).

${ }^{1} \mathrm{H}$ NMR (400 MHz, DMSO- $\left.d_{6}\right) \delta 10.80(\mathrm{~s}, 1 \mathrm{H}), 8.03$ (d, J=7.4 Hz, 1H), 7.19-7.38 (m, 9H), 7.13 $(\mathrm{d}, J=7.4 \mathrm{~Hz}, 1 \mathrm{H}), 6.87-6.89(\mathrm{~m}, 4 \mathrm{H}), 4.88(\mathrm{~d},, J=6.2 \mathrm{~Hz}, 1 \mathrm{H}), 4.65(\mathrm{~d},, J=5.0 \mathrm{~Hz}, 1 \mathrm{H}), 4.54$ $4.60(\mathrm{~m}, 1 \mathrm{H}), 4.04-4.09(\mathrm{~m}, 1 \mathrm{H}), 3.73-3.79(\mathrm{~m}, 7 \mathrm{H}), 3.10(\mathrm{dd}, J=5.1$ and $8.5 \mathrm{~Hz}, 1 \mathrm{H}), 2.95(\mathrm{dd}$, $J=6.3$ and $8.5 \mathrm{~Hz}, 1 \mathrm{H}), 2.08-2.18(\mathrm{~m}, 5 \mathrm{H}), 1.33-1.42(\mathrm{~m}, 1 \mathrm{H}) .{ }^{13} \mathrm{C}$ NMR $\left(126 \mathrm{MHz}, \mathrm{DMSO}-d_{6}\right)$ $\delta 170.9,161.7,158.0,155.4,148.0,145.1,135.8,135.8,129.7,127.8,127.7,126.6,113.1,95.1$, 85.2, 73.0, 71.7, 64.7, 63.6, 55.0, 43.3, 28.8, 24.3. HRMS calc. for $\mathrm{C}_{33} \mathrm{H}_{36} \mathrm{~N}_{3} \mathrm{O}_{7}[\mathrm{M}+\mathrm{H}]^{+} 586.2553$, found 586.2558.

\section{Synthesis of compounds 21 and 22}

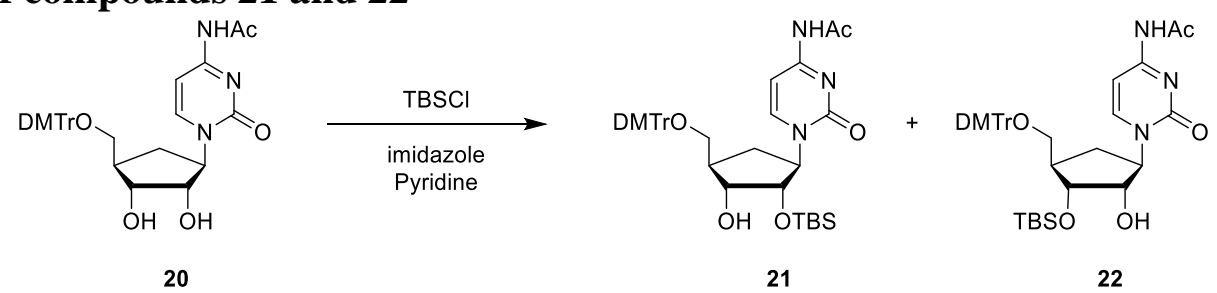

To a solution of compound 20 (2.1 g, $3.59 \mathrm{mmol})$ and imidazole (732 $\mathrm{mg}, 10.8 \mathrm{mmol})$ in pyridine (36 mL) was added tert-butyldimethylsilyl chloride $\left(594 \mathrm{mg}, 3.94 \mathrm{mmol}\right.$ ) at $0{ }^{\circ} \mathrm{C}$. The mixture was stirred at $0{ }^{\circ} \mathrm{C}$ for $30 \mathrm{~min}$ and then stirred at room temperature overnight. The reaction mixture was concentrated under vacuum. The residue was dissolved in ethyl acetate and washed with saturated $\mathrm{NaHCO}_{3}$ (aq.), water, brine, dried $\left(\mathrm{Na}_{2} \mathrm{SO}_{4}\right)$ and concentrated under vacuum. The crude residue was purified by column chromatography on silica gel (45-75\% ethyl acetate in hexane). Compound 21 was isolated as a white form (745 mg, 30\%), compound 22 was isolated as a white form (1.05 g, 42\%), and a mixture of compounds $\mathbf{2 1}$ and $\mathbf{2 2}$ was obtained as a white form (330 $\mathrm{mg}$, $13 \%)$.

Compound 21: ${ }^{1} \mathrm{H}$ NMR (400 MHz, DMSO- $\left.d_{6}\right) \delta 10.80(\mathrm{~s}, 1 \mathrm{H}), 8.03(\mathrm{~d}, J=7.4 \mathrm{~Hz}, 1 \mathrm{H}), 7.19-$ $7.40(\mathrm{~m}, 9 \mathrm{H}), 7.13(\mathrm{~d}, J=7.4 \mathrm{~Hz}, 1 \mathrm{H}), 6.87-6.89(\mathrm{~m}, 4 \mathrm{H}), 4.60-4.67(\mathrm{~m}, 1 \mathrm{H}), 4.37(\mathrm{~d}, J=5.7 \mathrm{~Hz}$, $1 \mathrm{H}), 4.31(\mathrm{dd}, J=5.8$ and $7.6 \mathrm{~Hz}, 1 \mathrm{H})$, ), 3.77-3.81 (m, 1H), $3.72(\mathrm{~s}, 6 \mathrm{H}), 3.14(\mathrm{dd}, J=6.1$ and $8.6 \mathrm{~Hz}, 1 \mathrm{H}), 2.97(\mathrm{dd}, J=6.0$ and $8.6 \mathrm{~Hz}, 1 \mathrm{H}), 2.03-2.18(\mathrm{~m}, 5 \mathrm{H}), 1.58-1.66(\mathrm{~m}, 1 \mathrm{H}), 0.76(\mathrm{~s}$, 9H), -0.05 (s, 3H), -0.13 (s, 3H). ${ }^{13} \mathrm{C}$ NMR (101 MHz, DMSO- $\left.d_{6}\right) \delta 170.9,161.7,158.0,155.2$, 148.7, 145.1, 135.8, 135.7, 129.7, 127.8, 127.7, 126.6, 113.1, 95.1, 85.2, 74.9, 71.8, 64.7, 64.5, 55.0, 43.5, 27.3, 25.6, 24.3, 17.8, -4.76, -5.14. HRMS calc. for $\mathrm{C}_{39} \mathrm{H}_{50} \mathrm{~N}_{3} \mathrm{O}_{7} \mathrm{Si}[\mathrm{M}+\mathrm{H}]^{+} 700.3418$, found 700.3412 .

Compound 22: ${ }^{1} \mathrm{H}$ NMR (400 MHz, DMSO- $\left.d_{6}\right) \delta 10.80(\mathrm{~s}, 1 \mathrm{H}), 8.02(\mathrm{~d}, J=7.4 \mathrm{~Hz}, 1 \mathrm{H}), 7.13-$ $7.37(\mathrm{~m}, 9 \mathrm{H}), 7.14(\mathrm{~d}, J=7.4 \mathrm{~Hz}, 1 \mathrm{H}), 6.86-6.88(\mathrm{~m}, 4 \mathrm{H}), 4.71(\mathrm{~d}, J=6.7 \mathrm{~Hz}, 1 \mathrm{H}), 4.51-4.58(\mathrm{~m}$, $1 \mathrm{H}), 4.05-4.10(\mathrm{~m}, 1 \mathrm{H}), 3.87(\mathrm{dd}, J=4.1$ and $4.8 \mathrm{~Hz}, 1 \mathrm{H}), 3.72(\mathrm{~s}, 6 \mathrm{H}), 3.13(\mathrm{dd}, J=4.8$ and 9.0 
$\mathrm{Hz}, 1 \mathrm{H}), 2.97(\mathrm{dd}, J=6.6$ and $9.0 \mathrm{~Hz}, 1 \mathrm{H}), 2.17-2.25(\mathrm{~m}, 1 \mathrm{H}), 2.08-2.08(\mathrm{~m}, 4 \mathrm{H}), 1.34-1.42(\mathrm{~m}$, $1 \mathrm{H}), 0.80(\mathrm{~s}, 9 \mathrm{H}),-0.01(\mathrm{~s}, 3 \mathrm{H}),-0.03(\mathrm{~s}, 3 \mathrm{H}) .{ }^{13} \mathrm{C}$ NMR (101 MHz, DMSO-d $\left.d_{6}\right) \delta 170.9,161.7$, 158.0, 155.4, 148.1 145.0, 135.7, 135.7, 129.7, 129.6, 127.8, 127.6, 126.6, 113.1, 95.1, 85.4, 73.91, 72.6, 64.6, 63.5, 55.0, 43.8, 28.4, 25.8, 24.3, 18.0, -4.41, -5.03. HRMS calc. for $\mathrm{C}_{39} \mathrm{H}_{50} \mathrm{~N}_{3} \mathrm{O}_{7} \mathrm{Si}[\mathrm{M}$ $+\mathrm{H}]^{+} 700.3418$, found 700.3414 .

\section{Synthesis of compound 3}
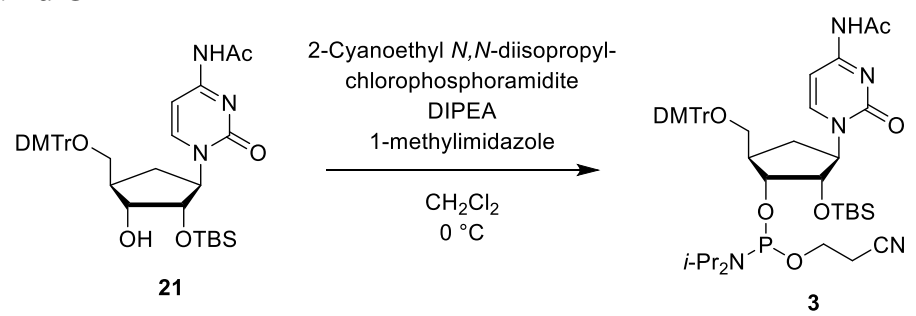

To a solution of compound 21 (720 mg, $1.03 \mathrm{mmol})$, 1-methylimidazole (8.20 uL, $0.103 \mathrm{mmol})$ and DIPEA $(0.538 \mathrm{~mL}, 3.09 \mathrm{mmol})$ in $\mathrm{CH}_{2} \mathrm{Cl}_{2}(10 \mathrm{~mL})$ was added dropwise 2-cyanoethyl $N, N$ diisopropylchlorophosphoramidite $(0.276 \mathrm{~mL}, 1.23 \mathrm{mmol})$ at $0{ }^{\circ} \mathrm{C}$ and the mixture was stirred at $0{ }^{\circ} \mathrm{C}$ for $3 \mathrm{~h}$. The reaction mixture was diluted with ethyl acetate and washed with saturated $\mathrm{NaHCO}_{3}$, brine, dried $\left(\mathrm{Na}_{2} \mathrm{SO}_{4}\right)$ and concentrated under vacuum. The crude residue was purified by column chromatography on silica gel (50\% ethyl acetate in hexane) to obtain compound $\mathbf{3}$ as a white form (774 mg, 84\%).

${ }^{1} \mathrm{H}$ NMR $\left(400 \mathrm{MHz}, \mathrm{CD}_{3} \mathrm{CN}\right) \delta 8.83(\mathrm{~s}, 1 \mathrm{H}), 7.64-7.66(\mathrm{~m}, 1 \mathrm{H}), 7.46-7.49(\mathrm{~m}, 2 \mathrm{H}), 7.28-7.36(\mathrm{~m}$, $6 \mathrm{H}), 7.20-7.25(\mathrm{~m}, 1 \mathrm{H}), 7.16-7.18(\mathrm{~m}, 1 \mathrm{H}), 6.84-6.89(\mathrm{~m}, 4 \mathrm{H}), 4.49-4.67(\mathrm{~m}, 2 \mathrm{H}), 4.05-4.13(\mathrm{~m}$, $1 \mathrm{H}), 3.56-3.95(\mathrm{~m}, 10 \mathrm{H}), 3.10-3.27(\mathrm{~m}, 2 \mathrm{H}), 2.34-2.68(\mathrm{~m}, 3 \mathrm{H}), 2.14-2.23(\mathrm{~m}, 1 \mathrm{H}), 2.11(\mathrm{~s}, 3 \mathrm{H})$, 1.80-1.90 (m, 1H), 1.10-1.18 (m, 12H), 0.79-0.80 (m, 9H), -0.02--0.01 (m, 3H), -0.13--0.12 (m, $3 \mathrm{H}) .{ }^{13} \mathrm{C}$ NMR $\left(101 \mathrm{MHz}, \mathrm{CD}_{3} \mathrm{CN}\right) \delta 171.7,163.0,162.9,159.7,156.7,156.6,150.2,149.6,146.4$, 146.3, 137.2, 137.2, 137.1, 137.1, 131.2, 131.2, 131.1, 129.2, 129.1, 128.9, 128.9, 127.8, 127.8, $119.7,119.5,114.1,114.1,96.4,96.4,87.1,77.2,77.2,76.0,75.9,75.8,75.7,75.6,75.6,66.8$, $66.5,65.5,65.3,60.0,59.9,59.0,58.8,56.0,56.0,44.3,44.2,43.8,43.7,43.4,43.3,27.4,26.9$, 26.4, 26.3, 26.3, 25.3, 25.2, 25.2, 25.2, 25.1, 25.1, 25.0, 25.0, 21.3, 21.3, 21.1, 21.1, 18.7, 18.7, 3.93, -3.95, -4.52, -4.55. ${ }^{31} \mathrm{P}$ NMR $\left(202 \mathrm{MHz}, \mathrm{CD}_{3} \mathrm{CN}\right) \delta 150.43,148.33$. HRMS calc. for $\mathrm{C}_{48} \mathrm{H}_{67} \mathrm{~N}_{5} \mathrm{O}_{8} \mathrm{PSi}[\mathrm{M}+\mathrm{H}]^{+} 900.4497$, found 900.4490 .

\section{Synthesis of compound 4}
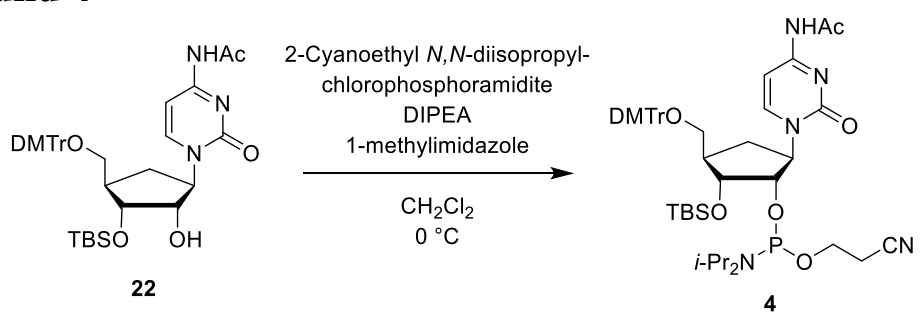

To a solution of compound 22 (1 g, $1.43 \mathrm{mmol}), 1$-methylimidazole $(11.4 \mathrm{uL}, 0.143 \mathrm{mmol})$ and DIPEA $(0.747 \mathrm{~mL}, 4.29 \mathrm{mmol})$ in $\mathrm{CH}_{2} \mathrm{Cl}_{2}(10 \mathrm{~mL})$ was added dropwise 2-cyanoethyl $N, N$ diisopropylchlorophosphoramidite $(0.382 \mathrm{~mL}, 1.71 \mathrm{mmol})$ at $0{ }^{\circ} \mathrm{C}$ and the mixture was stirred at $0{ }^{\circ} \mathrm{C}$ for $3 \mathrm{~h}$. The reaction mixture was diluted with ethyl acetate and washed with saturated $\mathrm{NaHCO}_{3}$, brine, dried $\left(\mathrm{Na}_{2} \mathrm{SO}_{4}\right)$ and concentrated under vacuum. The crude residue was purified 
by column chromatography on silica gel (50\% ethyl acetate in hexane) to give compound $\mathbf{4}$ as a white form (900 mg, 75\%).

${ }^{1} \mathrm{H}$ NMR $\left(400 \mathrm{MHz}, \mathrm{CD}_{3} \mathrm{CN}\right) \delta 8.70(\mathrm{~s}, 1 \mathrm{H}), 7.63(\mathrm{~d}, J=7.3 \mathrm{~Hz}, 0.8 \mathrm{H}), 7.59(\mathrm{~d}, J=7.3 \mathrm{~Hz}, 0.2 \mathrm{H})$, 7.36-7.40 (m, 2H), 7.22-7.26 (m, 6H), 7.13-7.18 (m, 1.8H), 7.06 (d, J = 7.3 Hz, 0.2H), 6.78-6.81 $(\mathrm{m}, 4 \mathrm{H}), 4.68-4.79(\mathrm{~m}, 1 \mathrm{H}), 4.38-4.44(\mathrm{~m}, 0.2 \mathrm{H}), 4.23-4.29(\mathrm{~m}, 0.8 \mathrm{H}), 4.10(\mathrm{dd}, J=1.8$ and 4.3 $\mathrm{Hz}, 0.2 \mathrm{H}), 4.00(\mathrm{dd}, J=1.6$ and $4.3 \mathrm{~Hz}, 0.8 \mathrm{H}), 3.70(\mathrm{~s}, 6 \mathrm{H}), 3.39-3.59(\mathrm{~m}, 4 \mathrm{H}), 3.01-3.18(\mathrm{~m}$, $2 \mathrm{H}), 2.41-2.48(\mathrm{~m}, 2 \mathrm{H}), 2.14-2.23(\mathrm{~m}, 2 \mathrm{H}), 2.03-2.04(\mathrm{~m}, 3 \mathrm{H}), 1.50-1.63(\mathrm{~m}, 1 \mathrm{H}), 0.88-1.03(\mathrm{~m}$, $12 \mathrm{H}), 0.81(\mathrm{~s}, 9 \mathrm{H}),-0.02-0.04(\mathrm{~m}, 6 \mathrm{H}) .{ }^{13} \mathrm{C} \mathrm{NMR}\left(101 \mathrm{MHz}, \mathrm{CD}_{3} \mathrm{CN}\right) \delta 171.7,163.1,163.0,159.7$, $156.8,156.7,149.7,149.1,146.4,146.4,137.2$, 137.2, 137.1, 131.1, 129.1, 129.1, 128.9, 127.8, 119.5, 114.1, 96.6, 96.4, 87.3, 87.1, 77.6, 77.4, 75.9, 75.8, 75.1, 75.1, 75.0, 74.9, 65.7, 65.3, 64.8, 64.8, 59.6, 59.4, 59.2, 58.2, 56.0, 45.3, 45.3, 44.0, 43.9, 28.3, 27.9, 26.4, 25.3, 25.2, 25.1, 25.1, 25.0, 24.9, 24.9, 21.1, 21.0, 20.8, 20.8, 18.8, 18.8, -3.87, -3.88, -3.93, -3.95, -4.11, -4.18. ${ }^{31} \mathrm{P}$ NMR $\left(162 \mathrm{MHz}, \mathrm{CD}_{3} \mathrm{CN}\right) \delta 150.51,148.94$. HRMS calc. for $\mathrm{C}_{48} \mathrm{H}_{67} \mathrm{~N}_{5} \mathrm{O}_{8} \mathrm{PSi}[\mathrm{M}+\mathrm{H}]^{+} 900.4497$, found 900.4492 .

\section{Synthesis of compound 23}

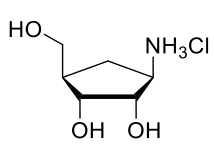

12

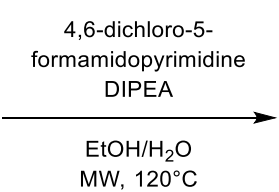

$\mathrm{MW}, 120^{\circ} \mathrm{C}$

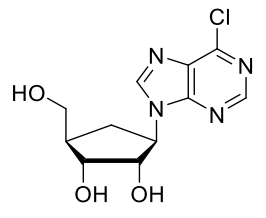

23

A mixture of $(1 R, 2 S, 3 R, 4 R)$-2,3-dihydroxy-4-(hydroxymthyl)-1-aminocyclo-pentane hydrochloride (12; $1 \mathrm{~g}, 5.45 \mathrm{mmol})$, 4,6-dichloro-5-formamidopyrimidine (1.25 g, $6.53 \mathrm{mmol})$ and DIPEA $(2.85 \mathrm{~mL}, 16.3 \mathrm{mmol})$ in $\mathrm{EtOH} / \mathrm{H}_{2} \mathrm{O}(1: 1 ; 16 \mathrm{~mL})$ was stirred and heated in a microwave reactor at $120^{\circ} \mathrm{C}$ for $6 \mathrm{~h}$. The reaction was cooled to room temperature and the solution was concentrated under vacuum. The crude residue was purified by column chromatography on silica gel (0-15\% $\mathrm{MeOH}$ in $\left.\mathrm{CH}_{2} \mathrm{Cl}_{2}\right)$ to obtain compound 23 as a white solid $(1.0 \mathrm{~g}, 64 \%)$.

${ }^{1} \mathrm{H}$ NMR $\left(500 \mathrm{MHz}, \mathrm{DMSO}-d_{6}\right) \delta 8.79(\mathrm{~s}, 1 \mathrm{H}), 8.76(\mathrm{~s}, 1 \mathrm{H}), 4.98(\mathrm{~d}, J=6.5 \mathrm{~Hz}, 1 \mathrm{H}), 4.83-4.88$ $(\mathrm{m}, 1 \mathrm{H}), 4.69-4.73(\mathrm{~m}, 2 \mathrm{H}), 4.35-4.39(\mathrm{~m}, 1 \mathrm{H}), 3.84-3.86(\mathrm{~m}, 1 \mathrm{H}), 3.43-3.53(\mathrm{~m}, 2 \mathrm{H}), 2.25-2.31$ $(\mathrm{m}, 1 \mathrm{H}), 2.03-2.09(\mathrm{~m}, 1 \mathrm{H}), 1.76-1.82(\mathrm{~m}, 1 \mathrm{H}) .{ }^{13} \mathrm{C}$ NMR (126 MHz, DMSO- $\left.d_{6}\right) \delta 152.1,151.1$, 149.0, 146.6, 131.3, 74.6, 71.4, 62.9, 60.1, 45.3, 28.8. HRMS calc. for $\mathrm{C}_{11} \mathrm{H}_{14} \mathrm{ClN}_{4} \mathrm{O}_{3}[\mathrm{M}+\mathrm{H}]^{+}$ 285.0754, found 285.0760.

\section{Synthesis of compound 24}

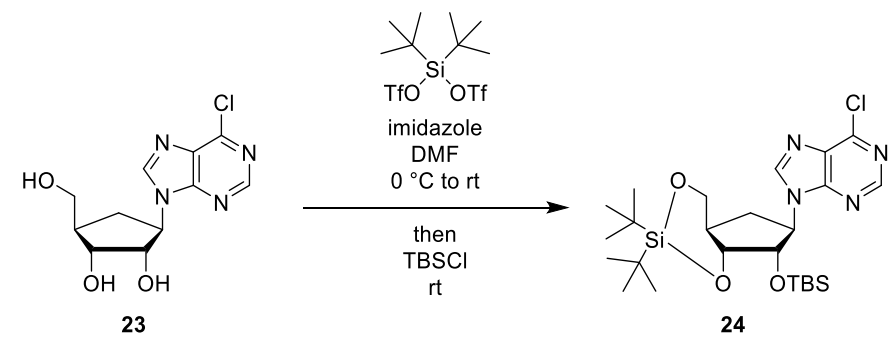

To a solution of compound 23 (900 mg, $3.16 \mathrm{mmol})$ in DMF (30 mL) was added dropwise di-tertbutylsilyl bis(trifluoromethanesulfonate) $(1.13 \mathrm{~mL}, 3.48 \mathrm{mmol})$ at $0{ }^{\circ} \mathrm{C}$ and the mixture was stirred at $0{ }^{\circ} \mathrm{C}$ for $30 \mathrm{~min}$. Imidazole $(1.08 \mathrm{~g}, 15.8 \mathrm{mmol})$ was added to the reaction mixture at $0{ }^{\circ} \mathrm{C}$. The mixture was further stirred at $\mathrm{rt}$ for $1 \mathrm{~h}$. tert-Butyldimethylsilyl chloride (953 $\mathrm{mg}, 6.32 \mathrm{mmol}$ ) was added to the reaction mixture and the mixture was stirred at room temperature for $12 \mathrm{~h}$. The 
reaction was quenched with saturated $\mathrm{NaHCO}_{3}$ (aq.) and diluted with diethyl ether. The organic layer was washed with water, brine, dried $\left(\mathrm{Na}_{2} \mathrm{SO}_{4}\right)$ and concentrated under vacuum. The crude residue was purified by column chromatography on silica gel (0-50\% ethyl acetate in hexane) to obtain compound 24 as a white form $(1.18 \mathrm{~g}, 69 \%)$.

${ }^{1} \mathrm{H}$ NMR $\left(400 \mathrm{MHz}, \mathrm{DMSO}-d_{6}\right) \delta 8.87(\mathrm{~s}, 1 \mathrm{H}), 8.77(\mathrm{~s}, 1 \mathrm{H}), 4.88(\mathrm{dt}, J=1.6$ and $9.0 \mathrm{~Hz}, 1 \mathrm{H}), 4.47$ (dd, $J=5.9$ and $11.0 \mathrm{~Hz}, 1 \mathrm{H}), 4.38(\mathrm{dd}, J=1.6$ and $5.9 \mathrm{~Hz}, 1 \mathrm{H}), 4.24(\mathrm{dd}, J=4.8$ and $10.1 \mathrm{~Hz}$, $1 \mathrm{H}), 3.94(\mathrm{t}, J=10.1 \mathrm{~Hz}, 1 \mathrm{H}), 2.35-2.45(\mathrm{~m}, 1 \mathrm{H}), 2.22-2.29(\mathrm{~m}, 1 \mathrm{H}), 1.64(\mathrm{dt}, J=9.0$ and 12.4 $\mathrm{Hz}, 1 \mathrm{H}), 1.05$ (s, 9H), 1.01 (s, 9H), $0.76(\mathrm{~s}, 9 \mathrm{H}),-0.04(\mathrm{~s}, 3 \mathrm{H}),-0.08(\mathrm{~s}, 3 \mathrm{H}) .{ }^{13} \mathrm{C} \mathrm{NMR}(126 \mathrm{MHz}$, DMSO-d $d_{6} \delta 151.4,151.3,149.2,146.6,131.1,77.6,76.7,68.4,62.3,41.1,28.9,27.3,26.9,25.6$, 22.2, 19.8, 17.9, -4.60, -5.40. HRMS calc. for $\mathrm{C}_{25} \mathrm{H}_{44} \mathrm{ClN}_{4} \mathrm{O}_{3} \mathrm{Si}_{2}[\mathrm{M}+\mathrm{H}]^{+} 539.2641$, found 539.2636 .

\section{Synthesis of compound 25}

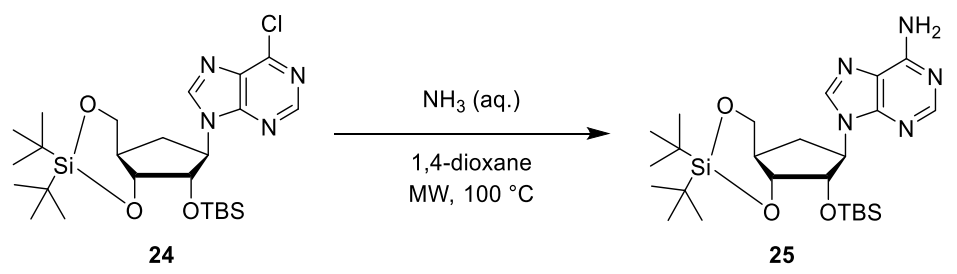

A mixture of compound 24 (780 $\mathrm{mg}, 1.45 \mathrm{mmol})$ in ammonium hydroxide solution (10 $\mathrm{mL})$ and 1,4-dioxane $(10 \mathrm{~mL})$ was stirred and heated in a microwave reactor at $100{ }^{\circ} \mathrm{C}$ for $3 \mathrm{~h}$. After the mixture was concentrated under vacuum, the crude residue was purified by column chromatography on silica gel (50-80\% ethyl acetate in hexane) to obtain compound $\mathbf{2 5}$ as a white form (736 mg, 98\%).

${ }^{1} \mathrm{H}$ NMR $\left(500 \mathrm{MHz}, \mathrm{DMSO}-d_{6}\right) \delta 8.25(\mathrm{~s}, 1 \mathrm{H}), 8.10(\mathrm{~s}, 1 \mathrm{H}), 7.20$ (brs, 2H), 4.73 (dt, $J=1.7$ and $9.2 \mathrm{~Hz}, 1 \mathrm{H}), 4.47(\mathrm{dd}, J=6.0$ and $11.0 \mathrm{~Hz}, 1 \mathrm{H}), 4.33(\mathrm{dd}, J=1.7$ and $6.0 \mathrm{~Hz}, 1 \mathrm{H}), 4.22(\mathrm{dd}, J=$ 4.8 and $10.2 \mathrm{~Hz}, 1 \mathrm{H}), 3.91(\mathrm{t}, J=10.2 \mathrm{~Hz}, 1 \mathrm{H}), 2.33-2.42(\mathrm{~m}, 1 \mathrm{H}), 2.13-2.19(\mathrm{~m}, 1 \mathrm{H}), 1.57(\mathrm{dt}, J$ $=9.2$ and $12.4 \mathrm{~Hz}, 1 \mathrm{H}), 1.04(\mathrm{~s}, 9 \mathrm{H}), 1.01(\mathrm{~s}, 9 \mathrm{H}), 0.76(\mathrm{~s}, 9 \mathrm{H}),-0.04(\mathrm{~s}, 3 \mathrm{H}),-0.06(\mathrm{~s}, 3 \mathrm{H}) .{ }^{13} \mathrm{C}$ NMR $\left(126 \mathrm{MHz}, \mathrm{DMSO}-d_{6}\right) \delta 156.6,152.9,149.7,140.7,119.7,78.3,77.5,69.1,62.4,41.9,29.8$, 27.9, 27.5, 26.3, 22.9, 20.4, 18.7, -3.95, -4.72. HRMS calc. for $\mathrm{C}_{25} \mathrm{H}_{46} \mathrm{~N}_{5} \mathrm{O}_{3} \mathrm{Si}_{2}[\mathrm{M}+\mathrm{H}]^{+} 520.3139$, found 520.3137 .

\section{Synthesis of compounds 26 and 27}

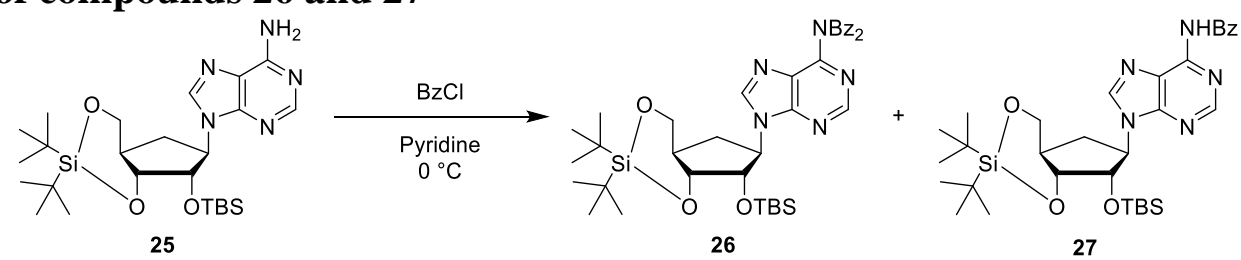

To a solution of compound 25 ( $1.4 \mathrm{~g}, 2.69 \mathrm{mmol})$ in pyridine $(25 \mathrm{~mL})$ was added dropwise benzoyl chloride $(0.344 \mathrm{~mL}, 2.96 \mathrm{mmol})$ and the mixture was stirred at $0{ }^{\circ} \mathrm{C}$ for 3 hours. The reaction was quenched with dry $\mathrm{MeOH}$ and concentrated under vacuum. The residue was dissolved in ethyl acetate and washed with saturated $\mathrm{NaHCO}_{3}$ (aq.), water, brine, dried $\left(\mathrm{Na}_{2} \mathrm{SO}_{4}\right)$ and concentrated under vacuum. The crude residue was purified by column chromatography on silica gel (25-50\% ethyl acetate in hexane) to obtain compound $\mathbf{2 6}$ as a white form (582 $\mathrm{mg}, 30 \%$ ) and compound 27 as a white form $(1.09 \mathrm{~g}, 65 \%)$.

Compound 26: ${ }^{1} \mathrm{H}$ NMR (500 MHz, DMSO-d $\left.d_{6}\right) 8.76(\mathrm{~s}, 1 \mathrm{H}), 8.68(\mathrm{~s}, 1 \mathrm{H}), 7.74-7.75(\mathrm{~m}, 4 \mathrm{H})$, 7.56-7.59 (m, 2H), 7.41-7.45 (m, 4H), $4.87(\mathrm{dt}, J=2.1$ and $9.5 \mathrm{~Hz}, 1 \mathrm{H}), 4.39(\mathrm{dd}, J=6.2$ and 10.9 
$\mathrm{Hz}, 1 \mathrm{H}), 4.33(\mathrm{dd}, J=2.1$ and $6.2 \mathrm{~Hz}, 1 \mathrm{H}), 4.24(\mathrm{dd}, J=4.8$ and $10.2 \mathrm{~Hz}, 1 \mathrm{H}), 3.92(\mathrm{t}, J=10.2$ $\mathrm{Hz}, 1 \mathrm{H}), 2.34-2.43(\mathrm{~m}, 1 \mathrm{H}), 2.21-2.26(\mathrm{~m}, 1 \mathrm{H}), 1.65(\mathrm{dt}, J=9.5$ and $12.2 \mathrm{~Hz}, 1 \mathrm{H}), 1.04(\mathrm{~s}, 9 \mathrm{H})$, $1.01(\mathrm{~s}, 9 \mathrm{H}), 0.71(\mathrm{~s}, 9 \mathrm{H}),-0.07(\mathrm{~s}, 3 \mathrm{H}),-0.14(\mathrm{~s}, 3 \mathrm{H}) .{ }^{13} \mathrm{C}$ NMR (101 MHz, DMSO-d $) \delta 171.9$, 152.5, 151.5, 150.8, 146.3, 133.4, 133.2, 128.9, 127.2, 77.5, 76.7, 68.3, 62.4, 41.4, 28.8, 27.3, 26.9, 25.6, 22.2, 19.8, 17.9, -4.64, -5.51. HRMS calc. for $\mathrm{C}_{39} \mathrm{H}_{54} \mathrm{~N}_{5} \mathrm{O}_{5} \mathrm{Si}_{2}[\mathrm{M}+\mathrm{H}]^{+} 728.3664$, found 728.3659 .

Compound 27: ${ }^{1} \mathrm{H}$ NMR $\left(500 \mathrm{MHz}, \mathrm{DMSO}-d_{6}\right) \delta 11.12(\mathrm{~s}, 1 \mathrm{H}), 8.72(\mathrm{~s}, 1 \mathrm{H}), 8.63(\mathrm{~s}, 1 \mathrm{H}), 8.02-$ $8.04(\mathrm{~m}, 2 \mathrm{H}), 7.62-7.65(\mathrm{~m}, 1 \mathrm{H}), 7.52-7.65(\mathrm{~m}, 2 \mathrm{H}), 4.88(\mathrm{dt}, J=1.6$ and $9.2 \mathrm{~Hz}, 1 \mathrm{H}), 4.49(\mathrm{dd}$, $J=5.9$ and $11.0 \mathrm{~Hz}, 1 \mathrm{H}), 4.40(\mathrm{dd}, J=1.6$ and $5.9 \mathrm{~Hz}, 1 \mathrm{H}), 4.25(\mathrm{dd}, J=4.9$ and $10.2 \mathrm{~Hz}, 1 \mathrm{H})$, $3.95(\mathrm{t}, J=10.2 \mathrm{~Hz}, 1 \mathrm{H}), 2.38-2.47(\mathrm{~m}, 1 \mathrm{H}), 2.22-2.28(\mathrm{~m}, 1 \mathrm{H}), 1.64(\mathrm{dt}, J=9.2$ and $12.4 \mathrm{~Hz}$, 1H), 1.06 (s, 9H), 1.02 (s, 9H), 0.77 (s, 9H), -0.02 (s, 3H), -0.06 (s, 3H). ${ }^{13} \mathrm{C}$ NMR (101 MHz, DMSO- $\left.d_{6}\right) \delta 165.5,151.9,151.3,150.2,143.8,133.4,132.3,128.4,125.6,77.7,76.8,68.4,62.0$, 41.2, 29.1, 27.3, 26.9, 25.7, 22.3, 19.8, 18.0, -4.56, -5.36. HRMS calc. for $\mathrm{C}_{32} \mathrm{H}_{50} \mathrm{~N}_{5} \mathrm{O}_{4} \mathrm{Si}_{2}[\mathrm{M}+$ $\mathrm{H}]^{+}$624.3401, found 624.3400 .

\section{Synthesis of compound 28}

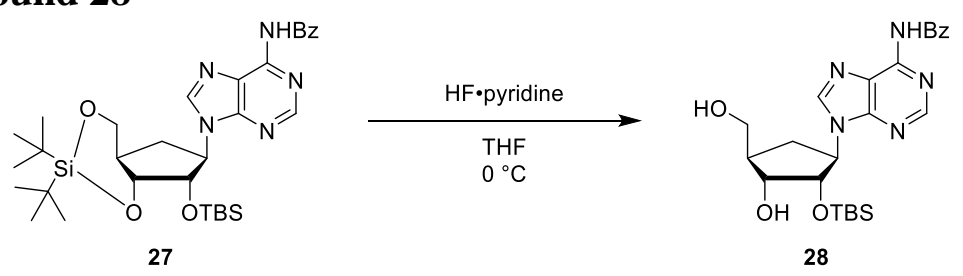

To a solution of compound $27(870 \mathrm{mg}, 1.39 \mathrm{mmol})$ in THF $(12 \mathrm{~mL})$ was added dropwise hydrogen fluoride pyridine $(0.485 \mathrm{~mL}, 5.58 \mathrm{mmol})$ at $0{ }^{\circ} \mathrm{C}$ and the mixture was stirred at $0{ }^{\circ} \mathrm{C}$ for $3 \mathrm{~h}$. The solvent was removed under vacuum. The crude residue was purified by column chromatography on silica gel (50-100\% ethyl acetate $(5 \% \mathrm{MeOH})$ in hexane) to obtain compound 28 as a white solid (562 mg, 83\%).

${ }^{1} \mathrm{H}$ NMR (400 MHz, DMSO-d6) $\delta 11.09$ (s, 1H), $8.71(\mathrm{~s}, 1 \mathrm{H}), 8.60$ (s, 1H), 8.02-8.04 (m, 2H), 7.61-7.65 (m, 1H), 7.52-7.55 (m, 2H), 4.92-4.99 (m, 1H), $4.79(\mathrm{t}, J=5.2 \mathrm{~Hz}, 1 \mathrm{H}), 4.45-4.50(\mathrm{~m}$, $2 \mathrm{H}), 3.85(\mathrm{dt}, J=2.6$ and $4.9 \mathrm{~Hz}, 1 \mathrm{H}), 3.47-3.59(\mathrm{~m}, 2 \mathrm{H}), 2.21-2.28(\mathrm{~m}, 1 \mathrm{H}), 2.06-2.14(\mathrm{~m}, 1 \mathrm{H})$, $1.91-1.99(\mathrm{~m}, 1 \mathrm{H}), 0.63(\mathrm{~s}, 9 \mathrm{H}),-0.18(\mathrm{~s}, 3 \mathrm{H}),-0.41(\mathrm{~s}, 3 \mathrm{H}),{ }^{13} \mathrm{C}$ NMR $\left(126 \mathrm{MHz}, \mathrm{DMSO}-d_{6}\right) \delta$ 165.5, 152.6, 151.0, 150.1, 143.9, 133.4, 132.3, 128.4, 126.0, 76.7, 71.5, 63.0, 60.0, 45.7, 27.9, 25.4, 17.6, -5.00, -5.52. HRMS calc. for $\mathrm{C}_{24} \mathrm{H}_{34} \mathrm{~N}_{5} \mathrm{O}_{4} \mathrm{Si}[\mathrm{M}+\mathrm{H}]^{+} 484.2380$, found 484.2375.

\section{Synthesis of compound 29}

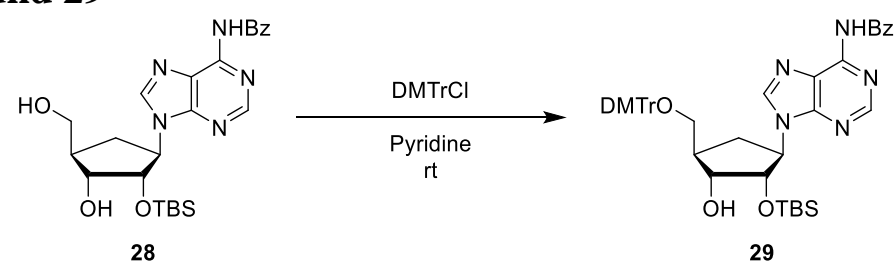

To a solution of compound $28(500 \mathrm{mg}, 1.03 \mathrm{mmol})$ in pyridine $(10 \mathrm{~mL})$ was added 4,4'dimethoxytriphenyl chloride $(525 \mathrm{mg}, 1.55 \mathrm{mmol})$ and the mixture was stirred at room temperature for $3 \mathrm{~h}$. The reaction was quenched with $\mathrm{MeOH}$ and concentrated under vacuum. The residue was dissolved in ethyl acetate. The organic layer was washed with saturated $\mathrm{NaHCO}_{3}$ (aq.), water, brine and dried $\left(\mathrm{Na}_{2} \mathrm{SO}_{4}\right)$ and concentrated under vacuum. The crude residue was purified by column chromatography on silica gel (50-70\% ethyl acetate in hexane) to obtain compound 29 as a white form $(775 \mathrm{mg}, 95 \%)$. 
${ }^{1} \mathrm{H}$ NMR (500 MHz, DMSO-d $) \delta 11.09(\mathrm{~s}, 1 \mathrm{H}), 8.58(\mathrm{~s}, 1 \mathrm{H}), 8.50(\mathrm{~s}, 1 \mathrm{H}), 8.02-8.04(\mathrm{~m}, 2 \mathrm{H})$, 7.61-7.64 (m, 1H), 7.52-7.55 (m, 2H), 7.46-7.48 (m, 2H), 7.30-7.33 (m, 6H), 7.21-7.24 (m, 1H), 6.89-6.91 (m, 4H), 4.89-4.94 (m, 1H), 4.54-4.58 (m, 2H), 3.87-3.89 (m, 1H), 3.19-3.22 (m, 1H), 3.09-3.11 (m, 1H), 2.22-2.28 (m, 3H), $0.62(\mathrm{~s}, 9 \mathrm{H}),-0.23(\mathrm{~s}, 3 \mathrm{H}),-0.45(\mathrm{~s}, 3 \mathrm{H}) .{ }^{13} \mathrm{C}$ NMR $(101$ MHz, DMSO- $\left.d_{6}\right) \delta 165.5,158.0,152.4,150.7,150.2,145.2,144.5,135.79,135.8,133.4,132.3$, 129.7, 128.4, 127.7, 127.7, 126.6, 126.3, 113.1, 85.2, 76.0, 71.8, 64.2, 60.6, 55.0, 43.5, 27.3, 25.43, 17.6, -5.12, -5.61. HRMS calc. for $\mathrm{C}_{45} \mathrm{H}_{52} \mathrm{~N}_{5} \mathrm{O}_{6} \mathrm{Si}[\mathrm{M}+\mathrm{H}]^{+}$786.3687, found 786.3690.

\section{Synthesis of compound 5}
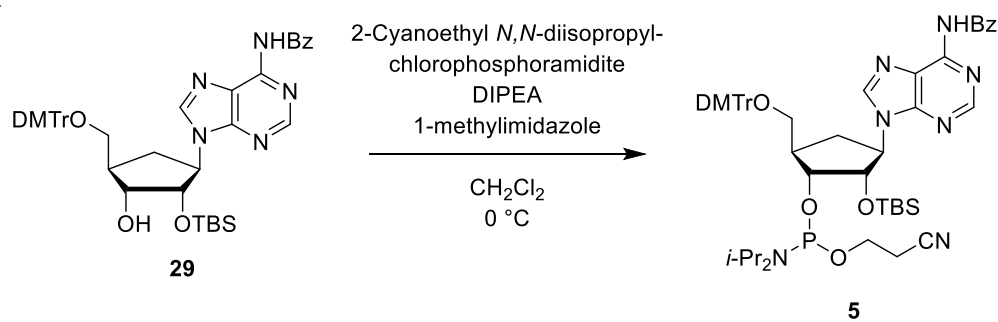

To a solution of compound 29 (600 mg, $0.763 \mathrm{mmol}), 1$-methylimidazole (6.08 $\mu \mathrm{L}, 76.3 \mathrm{umol})$ and DIPEA (0.399 mL, $2.29 \mathrm{mmol})$ in $\mathrm{CH}_{2} \mathrm{Cl}_{2}(7 \mathrm{~mL})$ was added dropwise 2-cyanoethyl $N, N$ diisopropylchlorophosphoramidite $(0.205 \mathrm{~mL}, 0.916 \mathrm{mmol})$ at $0{ }^{\circ} \mathrm{C}$ and the mixture was stirred at $0{ }^{\circ} \mathrm{C}$ for $3 \mathrm{~h}$. The reaction mixture was diluted with ethyl acetate and washed with saturated $\mathrm{NaHCO}_{3}$, water, brine, dried $\left(\mathrm{Na}_{2} \mathrm{SO}_{4}\right)$ and concentrated under vacuum. The crude residue was purified by column chromatography on silica gel (50\% ethyl acetate in hexane) to obtain compound 5 as a white form (630 $\mathrm{mg}, 84 \%)$.

${ }^{1} \mathrm{H}$ NMR $\left(500 \mathrm{MHz}, \mathrm{CD}_{3} \mathrm{CN}\right) \delta 9.46$ (brs, $\left.1 \mathrm{H}\right), 8.48-8.51(\mathrm{~m}, 1 \mathrm{H}), 8.10(\mathrm{~s}, 0.8 \mathrm{H}), 8.08(\mathrm{~s}, 0.2 \mathrm{H})$, 7.99-8.01 (m, 2H), 7.51-7.64 (m, 5H), 7.40-7.45 (m, 4H), 7.21-7.32 (m, 3H), 6.86-6.89 (m, 4H), $4.82-4.91(\mathrm{~m}, 1 \mathrm{H}), 4.71-4.77(\mathrm{~m}, 1 \mathrm{H}), 4.17(\mathrm{dd}, J=4.1$ and $12.5 \mathrm{~Hz}, 0.8 \mathrm{H}), 4.17(\mathrm{dd}, J=4.4$ and $9.3 \mathrm{~Hz}, 0.2 \mathrm{H}), 3.61-4.00(\mathrm{~m}, 10 \mathrm{H}), 3.31-3.38(\mathrm{~m}, 1 \mathrm{H}), 3.22-3.24(\mathrm{~m}, 1 \mathrm{H}), 2.35-2.76(\mathrm{~m}, 5 \mathrm{H})$, $1.12-1.22(\mathrm{~m}, 12 \mathrm{H}), 0.64-0.64(\mathrm{~m}, 9 \mathrm{H}),-0.25--0.23(\mathrm{~m}, 3 \mathrm{H}),-0.54--0.52(\mathrm{~m}, 3 \mathrm{H}) .{ }^{13} \mathrm{C} \mathrm{NMR}$ $\left(101 \mathrm{MHz}, \mathrm{CD}_{3} \mathrm{CN}\right) \delta 159.7,159.7,153.4,152.1,151.0,146.7,146.6,145.6,145.5,137.4,137.3$, 137.2, 137.2, 135.2, 133.5, 131.3, 131.2, 131.2, 129.7, 129.2, 129.1, 128.9, 127.8, 126.4, 126.4, $119.7,119.5,114.2,87.2,87.1,77.4,77.3,77.2,77.1,76.7,76.6,75.9,75.8,65.4,65.2,62.4,62.1$, 60.2, 60.1, 59.0, 58.8, 56.0, 44.4, 44.3, 43.8, 43.7, 43.5, 43.5, 43.4, 43.4, 27.6, 27.3, 26.2, 26.2, 26.2, 25.3, 25.3, 25.2, 25.2, 25.1, 25.0, 25.0, 21.4, 21.3, 21.2, 21.1, 18.6, 18.5, -4.26, -4.28, -4.30, $-4.34,-5.11 .{ }^{31} \mathrm{P}$ NMR $\left(202 \mathrm{MHz}, \mathrm{CD}_{3} \mathrm{CN}\right) \delta 150.17,147.90$. HRMS calc. for $\mathrm{C}_{54} \mathrm{H}_{69} \mathrm{~N}_{7} \mathrm{O}_{7} \mathrm{PSi}[\mathrm{M}$ $+\mathrm{H}]^{+}$986.4765, found 986.4752 . 


\section{Synthesis of compound 30}

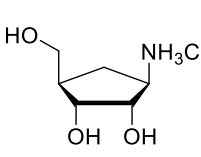

12

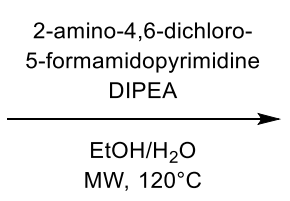

$\mathrm{MW}, 120^{\circ} \mathrm{C}$

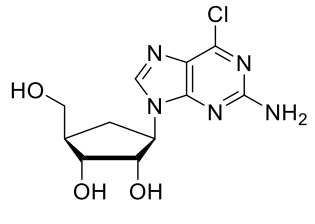

30

A mixture of $(1 R, 2 S, 3 R, 4 R)$-2,3-dihydroxy-4-(hydroxymthyl)-1-aminocyclo-pentane hydrochloride (12; $1 \mathrm{~g}, 5.44 \mathrm{mmol})$, 2-amino-4,6-dichloro-5-formamidopyrimidine (1.35 g, 6.53 mmol) and DIPEA $(2.85 \mathrm{~mL}, 16.3 \mathrm{mmol})$ in $\mathrm{EtOH} / \mathrm{H}_{2} \mathrm{O}(1: 1 ; 16 \mathrm{~mL})$ was stirred and heated in a microwave reactor at $120^{\circ} \mathrm{C}$ for $6 \mathrm{~h}$. The reaction was cooled to room temperature and the solution was concentrated under vacuum. The crude residue was purified by column chromatography on silica gel $\left(0-15 \% \mathrm{MeOH}\right.$ in $\left.\mathrm{CH}_{2} \mathrm{Cl}_{2}\right)$ to obtain compound $\mathbf{3 0}$ as a white solid $(1.25 \mathrm{~g}, 77 \%)$.

${ }^{1} \mathrm{H}$ NMR $\left(500 \mathrm{MHz}\right.$, DMSO- $\left.d_{6}\right) \delta 8.23(\mathrm{~s}, 1 \mathrm{H}), 6.83(\mathrm{~s}, 2 \mathrm{H}), 4.94(\mathrm{~d}, J=6.4 \mathrm{~Hz}, 1 \mathrm{H}), 4.60-4.70$ $(\mathrm{m}, 3 \mathrm{H}), 4.24-4.28(\mathrm{~m}, 1 \mathrm{H}), 3.79-3.81(\mathrm{~m}, 1 \mathrm{H}), 2.17-2.23(\mathrm{~m}, 1 \mathrm{H}), 1.97-2.03(\mathrm{~m}, 1 \mathrm{H}), 1.55-1.61$ $(\mathrm{m}, 1 \mathrm{H}) .{ }^{13} \mathrm{C}$ NMR $\left(101 \mathrm{MHz}, \mathrm{DMSO}-d_{6}\right) \delta 159.5,154.4,149.2,141.9,123.7,74.5,71.6,63.0$, 58.6, 45.2, 29.2. HRMS calc. for $\mathrm{C}_{11} \mathrm{H}_{15} \mathrm{ClN}_{5} \mathrm{O}_{3}[\mathrm{M}+\mathrm{H}]^{+} 300.0863$, found 300.0860 .

\section{Synthesis of compound 31}

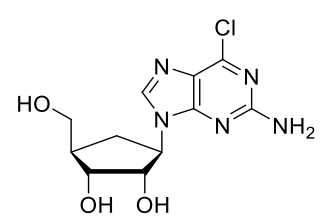

30

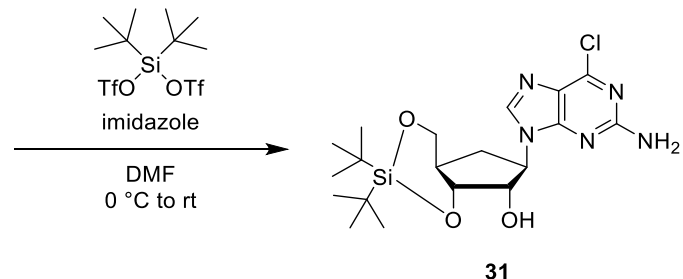

To a solution of compound $\mathbf{3 0}(1.63 \mathrm{~g}, 5.44 \mathrm{mmol})$ in DMF $(50 \mathrm{~mL})$ was added dropwide di-tertbutylsilyl bis(trifluoromethanesulfonate) $(1.77 \mathrm{~mL}, 5.44 \mathrm{mmol})$ at $0{ }^{\circ} \mathrm{C}$ and the mixture was stirred at $0{ }^{\circ} \mathrm{C}$ for $30 \mathrm{~min}$. Imidazole $(1.11 \mathrm{~g}, 16.3 \mathrm{mmol})$ was added to the reaction mixture at $0{ }^{\circ} \mathrm{C}$. The mixture was further stirred at $\mathrm{rt}$ for $1 \mathrm{~h}$. The reaction was quenched with saturated $\mathrm{NaHCO}_{3}$ (aq.) and diluted with diethyl ether. The organic layer was washed with water, brine, dried $\left(\mathrm{Na}_{2} \mathrm{SO}_{4}\right)$ and concentrated under vacuum. The crude residue was purified by column chromatography on silica gel (0-100\% ethyl acetate in hexane) to obtain compound $\mathbf{3 1}$ as a yellow form (1.75 g, 58\%). ${ }^{1} \mathrm{H}$ NMR $\left(500 \mathrm{MHz}, \mathrm{DMSO}-d_{6}\right) \delta 8.28(\mathrm{~s}, 1 \mathrm{H}), 6.83(\mathrm{~s}, 2 \mathrm{H}), 5.03(\mathrm{~d}, J=3.9 \mathrm{~Hz}, 1 \mathrm{H}), 4.60(\mathrm{t}, J=$ $8.9 \mathrm{~Hz}, 1 \mathrm{H}), 4.26(\mathrm{dd}, J=5.4$ and $11.0 \mathrm{~Hz}, 1 \mathrm{H}), 4.19(\mathrm{dd}, J=4.7$ and $11.0 \mathrm{~Hz}, 1 \mathrm{H}), 4.06(\mathrm{~m}, 1 \mathrm{H})$, $3.95(\mathrm{t}, J=11.0 \mathrm{~Hz}, 1 \mathrm{H}), 2.33-2.42(\mathrm{~m}, 1 \mathrm{H}), 2.13-2.19(\mathrm{~m}, 1 \mathrm{H}), 1.52(\mathrm{dt}, J=8.9$ and $12.5 \mathrm{~Hz}$, $1 \mathrm{H}), 1.03(\mathrm{~s}, 9 \mathrm{H}), 1.00(\mathrm{~s}, 9 \mathrm{H}) .{ }^{13} \mathrm{C}$ NMR $\left(126 \mathrm{MHz}, \mathrm{DMSO}-d_{6}\right) \delta 159.5,153.7,149.4,142.1$, 123.4, 78.4, 75.1, 68.1, 59.6, 40.8, 28.8, 27.3, 27.1, 22.2, 19.9. HRMS calc. for $\mathrm{C}_{19} \mathrm{H}_{31} \mathrm{ClN}_{5} \mathrm{O}_{3} \mathrm{Si}$ $[\mathrm{M}+\mathrm{H}]^{+} 440.1885$, found 440.1884

\section{Synthesis of compound 32}
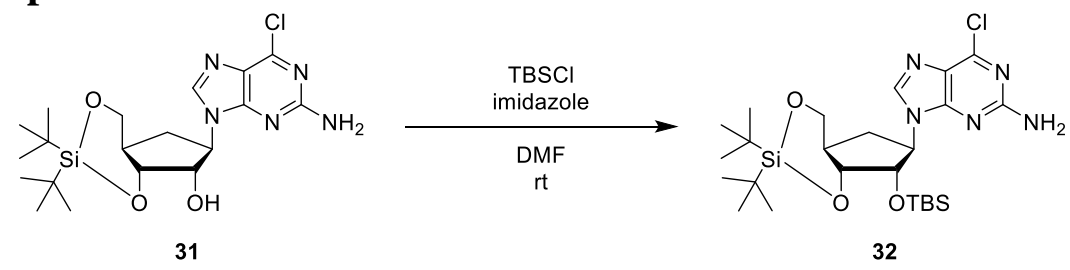

To a solution of compound $\mathbf{3 1}$ (400 $\mathrm{mg}, 0.909 \mathrm{mmol}$ ) and imidazole (186 mg, $2.73 \mathrm{mmol}$ ) in DMF $(9 \mathrm{~mL})$ was added tert-butyldimethylsilyl chloride $(206 \mathrm{mg}, 1.36 \mathrm{mmol})$ and the mixture was 
stirred at rt overnight. The reaction mixture was quenched with saturated $\mathrm{NaHCO}_{3}$ (aq.) and diluted with diethyl ether. The organic layer was washed with water, brine, dried $\left(\mathrm{Na}_{2} \mathrm{SO}_{4}\right)$ and concentrated under vacuum. The crude residue was purified by column chromatography on silica gel (0-25\% ethyl acetate in hexane) to obtain compound 32 as a white form (339 $\mathrm{mg}, 67 \%)$.

${ }^{1} \mathrm{H}$ NMR (400 MHz, DMSO-d6) $\delta 8.29(\mathrm{~s}, 1 \mathrm{H}), 6.79(\mathrm{~s}, 2 \mathrm{H}), 4.63(\mathrm{t}, J=9.2 \mathrm{~Hz}, 1 \mathrm{H}), 4.27-4.32$ $(\mathrm{m}, 2 \mathrm{H}), 4.21(\mathrm{dd}, J=4.8$ and $10.1 \mathrm{~Hz}, 1 \mathrm{H}), 3.92(\mathrm{t}, J=10.1 \mathrm{~Hz}, 1 \mathrm{H}), 2.28-2.39(\mathrm{~m}, 1 \mathrm{H}), 2.10$ $2.16(\mathrm{~m}, 1 \mathrm{H}), 1.53(\mathrm{dt}, J=9.2$ and $12.4 \mathrm{~Hz}, 1 \mathrm{H}), 1.03(\mathrm{~s}, 9 \mathrm{H}), 0.99(\mathrm{~s}, 9 \mathrm{H}), 0.75(\mathrm{~s}, 9 \mathrm{H}),-0.05(\mathrm{~s}$, $3 \mathrm{H}),-0.06(\mathrm{~s}, 3 \mathrm{H}) .{ }^{13} \mathrm{C}$ NMR $\left(126 \mathrm{MHz}, \mathrm{DMSO}-d_{6}\right) \delta 160.0,154.2,149.9,142.6,123.9,78.1,77.2$, $68.8,62.0,41.8,29.3,27.8,27.4,26.2,22.7,20.3,18.5,-4.09,-4.83$. HRMS calc. for $\mathrm{C}_{25} \mathrm{H}_{45} \mathrm{ClN}_{5} \mathrm{O}_{3} \mathrm{Si}_{2}[\mathrm{M}+\mathrm{H}]^{+}$554.2749, found 554.2750.

\section{Synthesis of compound 33}
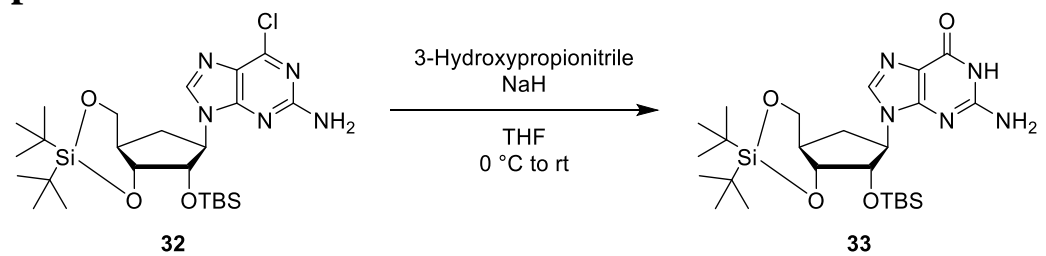

3-Hydroxypropionitrile $(0.555 \mathrm{~mL}, 8.12 \mathrm{mmol})$ was dissolved in THF $(10 \mathrm{~mL})$ and cooled to $0{ }^{\circ} \mathrm{C}$. Sodium hydride $(60 \%$ in mineral oil; $311 \mathrm{mg}, 8.12 \mathrm{mmol})$ was added in portions at $0{ }^{\circ} \mathrm{C}$ and the mixture was stirred at room temperature for $30 \mathrm{~min}$ and cooled to $0{ }^{\circ} \mathrm{C}$. A solution of compound $32(1 \mathrm{~g}, 1.80 \mathrm{mmol})$ in THF $(5 \mathrm{~mL})$ was added dropwise at $0{ }^{\circ} \mathrm{C}$ and the mixture was stirred at room temperature. After $12 \mathrm{~h}$ the reaction was quenched by addition of saturated $\mathrm{NH}_{4} \mathrm{Cl}$ (aq.). The reaction mixture was extracted with $\mathrm{CH}_{2} \mathrm{Cl}_{2}$ and ethyl acetate. The combined organic layers were washed with brine, dried $\left(\mathrm{Na}_{2} \mathrm{SO}_{4}\right)$ and concentrated under vacuum. The crude residue was purified by column chromatography on silica gel $\left(0-10 \% \mathrm{MeOH}\right.$ in $\left.\mathrm{CH}_{2} \mathrm{Cl}_{2}\right)$ to obtain compound $\mathbf{3 3}$ as a white solid (921 $\mathrm{mg}, 95 \%)$.

${ }^{1} \mathrm{H}$ NMR (500 MHz, DMSO-d $) \delta 10.53(\mathrm{~s}, 1 \mathrm{H}), 7.83(\mathrm{~s}, 1 \mathrm{H}), 6.26(\mathrm{~s}, 2 \mathrm{H}), 4.51-4.55(\mathrm{~m}, 1 \mathrm{H})$, $4.18-4.27(\mathrm{~m}, 3 \mathrm{H}), 3.89(\mathrm{t}, J=10.6 \mathrm{~Hz}, 1 \mathrm{H}), 2.26-2.35(\mathrm{~m}, 1 \mathrm{H}), 2.05-2.11(\mathrm{~m}, 1 \mathrm{H}), 1.45(\mathrm{dt}, J=$ 9.8 and $12.4 \mathrm{~Hz}, 1 \mathrm{H}), 1.03(\mathrm{~s}, 9 \mathrm{H}), 1.00(\mathrm{~s}, 9 \mathrm{H}), 0.77(\mathrm{~s}, 9 \mathrm{H}),-0.04(\mathrm{~s}, 6 \mathrm{H}) .{ }^{13} \mathrm{C} \mathrm{NMR}(126 \mathrm{MHz}$, DMSO- $\left.d_{6}\right) \delta 156.7,153.2,150.8,136.2,116.6,77.6,76.9,68.3,61.1,41.4,29.2,27.3,26.9,25.6$, 22.2, 19.8, 18.0, -4.65, -5.36. HRMS calc. for $\mathrm{C}_{25} \mathrm{H}_{46} \mathrm{~N}_{5} \mathrm{O}_{4} \mathrm{Si}_{2}[\mathrm{M}+\mathrm{H}]^{+}$536.3088, found 536.3083.

\section{Synthesis of compound 34}

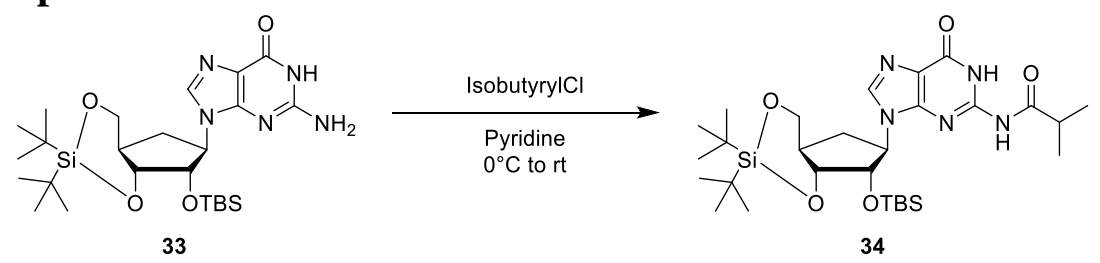

To a solution of compound $\mathbf{3 3}(2 \mathrm{~g}, 3.73 \mathrm{mmol})$ in pyridine $(35 \mathrm{~mL})$ was added dropwise isobutyryl chloride $(0.468 \mathrm{~mL}, 4.48 \mathrm{mmol})$ at $0{ }^{\circ} \mathrm{C}$ and the mixture was stirred at room temperature for 3 h. The reaction was quenched with dry $\mathrm{MeOH}$ and concentrated under vacuum. The residue was dissolved in ethyl acetate and washed with saturated $\mathrm{NaHCO}_{3}$ (aq.), water and brine and concentrated under vacuum. The crude residue was purified by column chromatography on silica gel (50-75\% ethyl acetate in hexane) to obtain compound 34 as a brown form $(1.82 \mathrm{~g}, 81 \%)$. ${ }^{1} \mathrm{H}$ NMR $\left(500 \mathrm{MHz}, \mathrm{DMSO}-d_{6}\right) \delta 12.01(\mathrm{~s}, 1 \mathrm{H}), 11.42(\mathrm{~s}, 1 \mathrm{H}), 8.19(\mathrm{~s}, 1 \mathrm{H}), 4.62-4.66(\mathrm{~m}, 1 \mathrm{H})$, 4.19-4.30 (m, 3H), 3.91 (t, $J=10.7 \mathrm{~Hz}, 1 \mathrm{H}), 2.77$ (sep, $J=6.8 \mathrm{~Hz}, 1 \mathrm{H}), 2.26-2.35$ (m, 1H), 2.09- 
$2.15(\mathrm{~m}, 1 \mathrm{H}), 1.10-1.11(\mathrm{~m}, 6 \mathrm{H}), 1.03(\mathrm{~s}, 9 \mathrm{H}), 0.99$ (s, 9H), $0.73(\mathrm{~s}, 9 \mathrm{H}),-0.05$ (s, 3H), -0.07 (s, $3 \mathrm{H}) .{ }^{13} \mathrm{C}$ NMR $\left(126 \mathrm{MHz}, \mathrm{DMSO}-d_{6}\right) \delta 179.6,154.7,148.2,147.4,138.0,119.8,77.3,76.7,68.1$, 61.5, 41.5, 34.5, 29.4, 27.1, 26.7, 25.4, 22.0, 19.6, 18.6, 18.5, 17.8, -4.85, -5.61. HRMS calc. for $\mathrm{C}_{29} \mathrm{H}_{52} \mathrm{~N}_{5} \mathrm{O}_{5} \mathrm{Si}_{2}[\mathrm{M}+\mathrm{H}]^{+}$606.3507, found 606.3511 .

\section{Synthesis of compound 35}

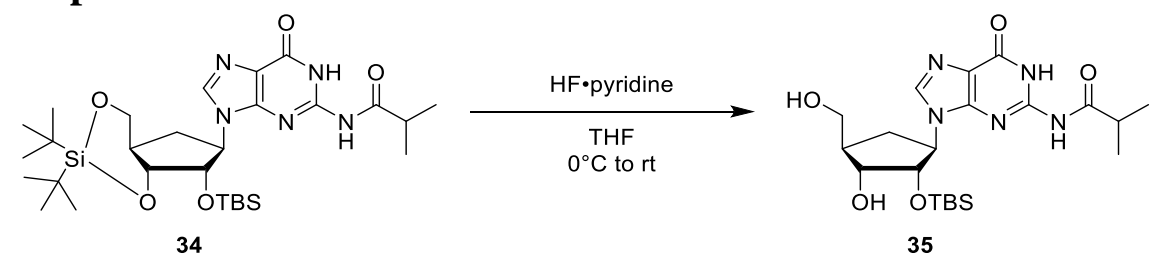

To a solution of compound $\mathbf{3 4}(1.8 \mathrm{~g}, 2.97 \mathrm{mmol})$ and pyridine $(5 \mathrm{~mL})$ in THF $(20 \mathrm{~mL})$ was added dropwise hydrogen fluoride pyridine $(1.03 \mathrm{~mL}, 11.9 \mathrm{mmol})$ at $0{ }^{\circ} \mathrm{C}$ and the mixture was stirred at $0{ }^{\circ} \mathrm{C}$ for $30 \mathrm{~min}$. The reaction mixture was poured into $\mathrm{CH}_{2} \mathrm{Cl}_{2}(50 \mathrm{~mL})$ to obtain the brown precipitate. The precipitate was filtered through $0.2-\mu \mathrm{m}$ syringe filter and washed with THF and $\mathrm{CH}_{2} \mathrm{Cl}_{2}$ to obtain compound $\mathbf{3 5}$ as a brown solid $(1.27 \mathrm{~g}, 92 \%)$.

${ }^{1} \mathrm{H}$ NMR (500 MHz, DMSO-d6) $\delta 12.00(\mathrm{~s}, 1 \mathrm{H}), 11.62(\mathrm{~s}, 1 \mathrm{H}), 8.17(\mathrm{~s}, 1 \mathrm{H}), 4.74-4.83(\mathrm{~m}, 2 \mathrm{H})$, $4.31(\mathrm{~d}, J=3.9 \mathrm{~Hz}, 1 \mathrm{H}), 4.26(\mathrm{dd}, J=5.1$ and $10.1 \mathrm{~Hz}, 1 \mathrm{H}), 3.78-3.81(\mathrm{~m}, 1 \mathrm{H}), 3.44-3.53(\mathrm{~m}$, 2H), $2.76(\mathrm{sep}, J=6.9 \mathrm{~Hz}, 1 \mathrm{H}), 2.16-2.22(\mathrm{~m}, 1 \mathrm{H}), 2.01-2.07(\mathrm{~m}, 1 \mathrm{H}), 1.63-1.69(\mathrm{~m}, 1 \mathrm{H}), 1.10-$ $1.11(\mathrm{~m}, 6 \mathrm{H}), 0.65(\mathrm{~s}, 9 \mathrm{H}),-0.13(\mathrm{~s}, 3 \mathrm{H}),-0.35(\mathrm{~s}, 3 \mathrm{H}) .{ }^{13} \mathrm{C}$ NMR $\left(126 \mathrm{MHz}, \mathrm{DMSO}-d_{6}\right) \delta 180.1$, 154.8, 149.1, 147.5, 137.9, 120.0, 77.8, 71.7, 63.0, 58.0, 45.5, 34.6, 28.7, 25.4, 18.8, 18.8, 17.5, 5.03, -5.65. HRMS calc. for $\mathrm{C}_{21} \mathrm{H}_{36} \mathrm{~N}_{5} \mathrm{O}_{5} \mathrm{Si}[\mathrm{M}+\mathrm{H}]^{+} 466.2486$, found 466.2491.

\section{Synthesis of compound 36}

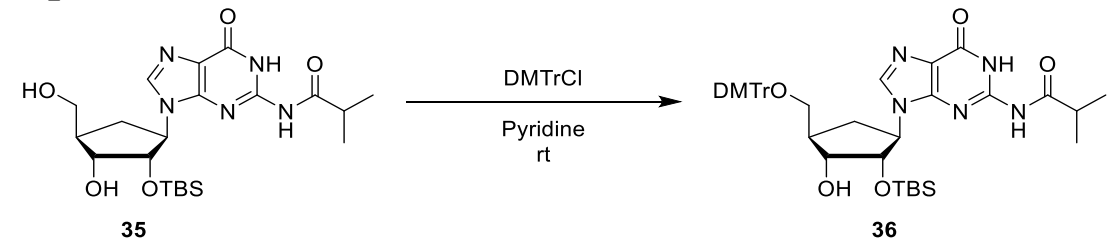

To a solution of compound $35(1.1 \mathrm{~g}, 2.36 \mathrm{mmol})$ in pyridine $(20 \mathrm{~mL})$ was added 4,4'dimethoxytriphenyl chloride $(1.2 \mathrm{~g}, 3.54 \mathrm{mmol})$ and the mixture was stirred at room temperature for $3 \mathrm{~h}$. The reaction was quenched with $\mathrm{MeOH}$ and concentrated under vacuum. The residue was dissolved in ethyl acetate and saturated $\mathrm{NaHCO}_{3}$ (aq.). The organic layer was washed with water and brine and dried $\left(\mathrm{Na}_{2} \mathrm{SO}_{4}\right)$ and concentrated under vacuum. The crude residue was purified by column chromatography on silica gel (50-70\% ethyl acetate in hexane) to obtain compound 36 as a white form (1.81 g, quant).

${ }^{1} \mathrm{H}$ NMR (500 MHz, DMSO-d $\left.d_{6}\right) \delta 12.00(\mathrm{~s}, 1 \mathrm{H}), 11.62(\mathrm{~s}, 1 \mathrm{H}), 8.09(\mathrm{~s}, 1 \mathrm{H}), 7.39-7.41(\mathrm{~m}, 2 \mathrm{H})$, $7.21-7.33(\mathrm{~m}, 7 \mathrm{H}), 6.88-6.90(\mathrm{~m}, 4 \mathrm{H}), 4.76-4.82(\mathrm{~m}, 1 \mathrm{H}), 4.46(\mathrm{~d}, J=4.6 \mathrm{~Hz}, 1 \mathrm{H}), 4.18(\mathrm{dd}, J=$ 5.4 and $9.3 \mathrm{~Hz}, 1 \mathrm{H}), 3.80-3.83(\mathrm{~m}, 1 \mathrm{H}), 3.73(\mathrm{~s}, 6 \mathrm{H}), 3.16(\mathrm{dd}, J=6.7$ and $9.2 \mathrm{~Hz}, 1 \mathrm{H}), 3.06(\mathrm{dd}$, $J=5.7$ and $9.2 \mathrm{~Hz}, 1 \mathrm{H}), 2.74(\mathrm{sep}, J=7.3 \mathrm{~Hz}, 1 \mathrm{H}), 2.16-2.26(\mathrm{~m}, 2 \mathrm{H}), 1.63-1.69(\mathrm{~m}, 1 \mathrm{H}), 1.09-$ $1.10(\mathrm{~m}, 6 \mathrm{H}), 0.65(\mathrm{~s}, 9 \mathrm{H}),-0.14(\mathrm{~s}, 3 \mathrm{H}),-0.34(\mathrm{~s}, 3 \mathrm{H}) .{ }^{13} \mathrm{C}$ NMR $\left(126 \mathrm{MHz}, \mathrm{DMSO}-d_{6}\right) \delta 180.0$, 158.0, 158.0, 154.8, 149.2, 147.6, 145.1, 137.5, 135.7, 135.6, 129.7, 129.7, 127.8, 127.6, 126.6, 119.9, 113.1, 85.3, 77.8, 71.7, 64.6, 57.8, 55.0, 43.6, 34.6, 28.9, 25.3, 18.8, 18.7, 17.5, -5.06, -5.71. HRMS calc. for $\mathrm{C}_{42} \mathrm{H}_{54} \mathrm{~N}_{5} \mathrm{O}_{7} \mathrm{Si}[\mathrm{M}+\mathrm{H}]^{+} 768.3793$, found 768.3781 . 


\section{Synthesis of compound 6}

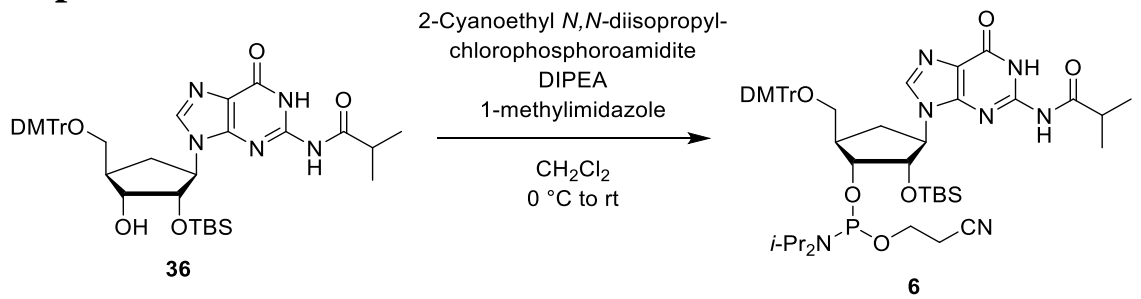

To a solution of compound 32 (1.85 g, $2.41 \mathrm{mmol})$, 1-methylimidazole (19.2 $\mathrm{uL}, 0.241 \mathrm{mmol})$ and DIPEA (1.26 mL, $7.23 \mathrm{mmol})$ in $\mathrm{CH}_{2} \mathrm{Cl}_{2}(20 \mathrm{~mL})$ was added dropwise 2-cyanoethyl $N, N$ diisopropylchlorophosphoramidite $(1.08 \mathrm{~mL}, 4.82 \mathrm{mmol})$ at $0{ }^{\circ} \mathrm{C}$ and the mixture was stirred at $0{ }^{\circ} \mathrm{C}$ for $3 \mathrm{~h}$. The reaction mixture was diluted with ethyl acetate and washed with saturated $\mathrm{NaHCO}_{3}$, water, brine, dried $\left(\mathrm{Na}_{2} \mathrm{SO}_{4}\right)$ and concentrated under vacuum. The crude residue was purified by column chromatography on silica gel (50\% ethyl acetate in hexane) to obtain compound $\mathbf{3 3}$ as a white form $(1.89 \mathrm{~g}, 81 \%)$.

${ }^{1} \mathrm{H}$ NMR $\left(500 \mathrm{MHz}, \mathrm{CD}_{3} \mathrm{CN}\right) \delta 7.71(\mathrm{~s}, 0.8 \mathrm{H}), 7.67(\mathrm{~s}, 0.2 \mathrm{H}), 7.54-7.58(\mathrm{~m}, 2 \mathrm{H}), 7.39-7.44(\mathrm{~m}$, 4H), 7.30-7.34 (m, 2H), 7.22-7.26 (m, 1H), 6.86-6.90 (m, 4H), 4.69-4.82 (m, 1H), $4.65(\mathrm{dd}, J=$ 4.7 and $9.7 \mathrm{~Hz}, 0.2 \mathrm{H}), 4.58(\mathrm{dd}, J=4.3$ and $10.0 \mathrm{~Hz}, 0.8 \mathrm{H}), 3.93-4.04(\mathrm{~m}, 2 \mathrm{H}), 3.84-3.91(\mathrm{~m}, 1 \mathrm{H})$, 3.75-3.76 (m, 6H), 3.53-3.64 (m, 2H), $3.33(\mathrm{dd}, J=3.7$ and $9.3 \mathrm{~Hz}, 0.2 \mathrm{H}), 3.25(\mathrm{dd}, J=3.9$ and $9.3 \mathrm{~Hz}, 0.8 \mathrm{H}), 3.11-3.15(\mathrm{~m}, 1 \mathrm{H}), 2.32-2.74(\mathrm{~m}, 5 \mathrm{H}), 1.95-2.02(\mathrm{~m}, 1 \mathrm{H}), 1.14-1.18(\mathrm{~m}, 7 \mathrm{H})$, $1.01-1.02(\mathrm{~m}, 5 \mathrm{H}), 0.86-0.90(\mathrm{~m}, 6 \mathrm{H}), 0.73(\mathrm{~s}, 9 \mathrm{H}),-0.12--0.11(\mathrm{~m}, 3 \mathrm{H}),-0.35--0.33(\mathrm{~m}, 3 \mathrm{H})$. ${ }^{13} \mathrm{C}$ NMR $\left(126 \mathrm{MHz}, \mathrm{CD}_{3} \mathrm{CN}\right) \delta 180.3,180.3,159.8,156.4,150.2,150.0,148.2,148.1,146.7$, $146.6,140.5,140.0,137.6,137.4,137.2,131.1,129.1,129.1,129.0,128.0,123.0,122.8,120.0$, 119.5, 114.4, 114.3, 87.3, 87.1, 78.6, 78.6, 77.5, 77.5, 77.3, 77.2, 75.8, 75.7, 65.5, 65.5, 62.2, 61.2, $60.1,60.0,58.9,58.7,56.0,44.3,44.2,43.7,43.6,43.5,43.5,43.4,43.4,36.8,28.2,27.8,26.3$, 26.2, 26.2, 25.3, 25.2, 25.2, 25.1, 25.1, 25.0, 21.3, 21.3, 21.1, 21.1, 19.0, 18.9, 18.6, 18.6, -4.13, $4.15,-4.19,-4.22,-5.03,-5.05 .{ }^{31} \mathrm{P}$ NMR $\left(202 \mathrm{MHz}, \mathrm{CD}_{3} \mathrm{CN}\right) \delta 149.61,147.68$. HRMS calc. for $\mathrm{C}_{51} \mathrm{H}_{71} \mathrm{~N}_{7} \mathrm{O}_{8} \mathrm{PSi}[\mathrm{M}+\mathrm{H}]^{+}$968.4871, found 968.4882 . 
NMR spectra for the new compounds

${ }^{1} \mathrm{H}$ NMR spectrum (400 MHz) of compound 8 in DMSO- $d_{6}$

I

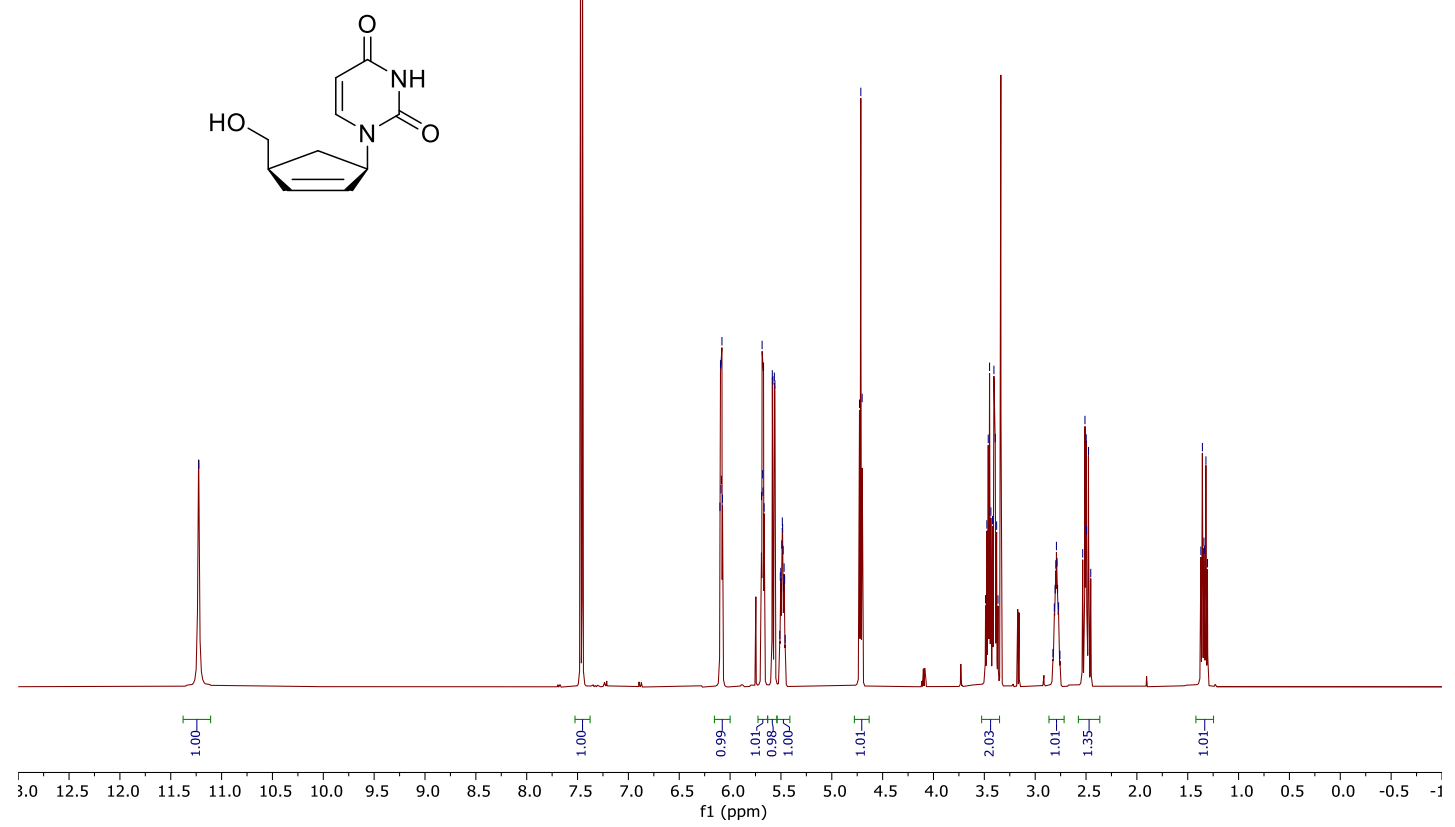

${ }^{13} \mathrm{C}$ NMR spectrum (101 MHz) of compound 8 in DMSO- $d_{6}$

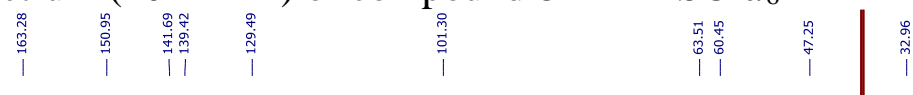
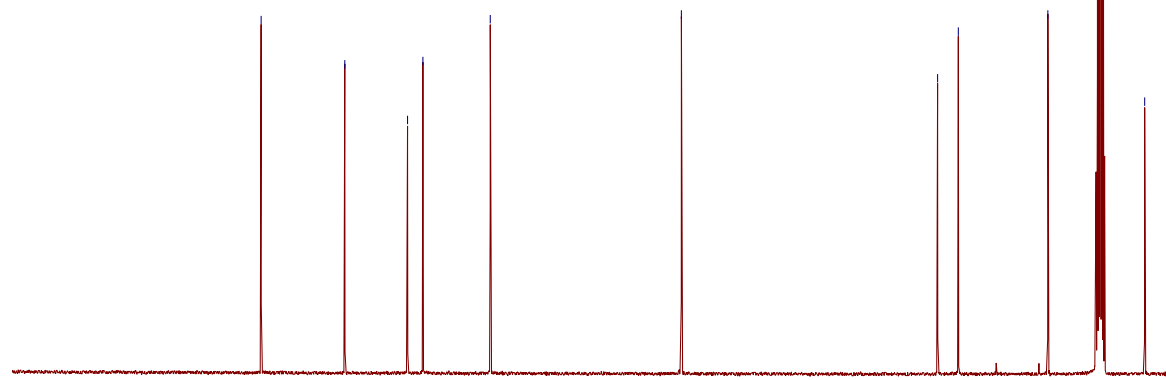

oo

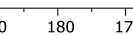

$160 \quad 150$

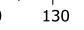

110 $\begin{array}{cc}100 & 90 \\ \text { f1 } & (\mathrm{ppm})\end{array}$ 


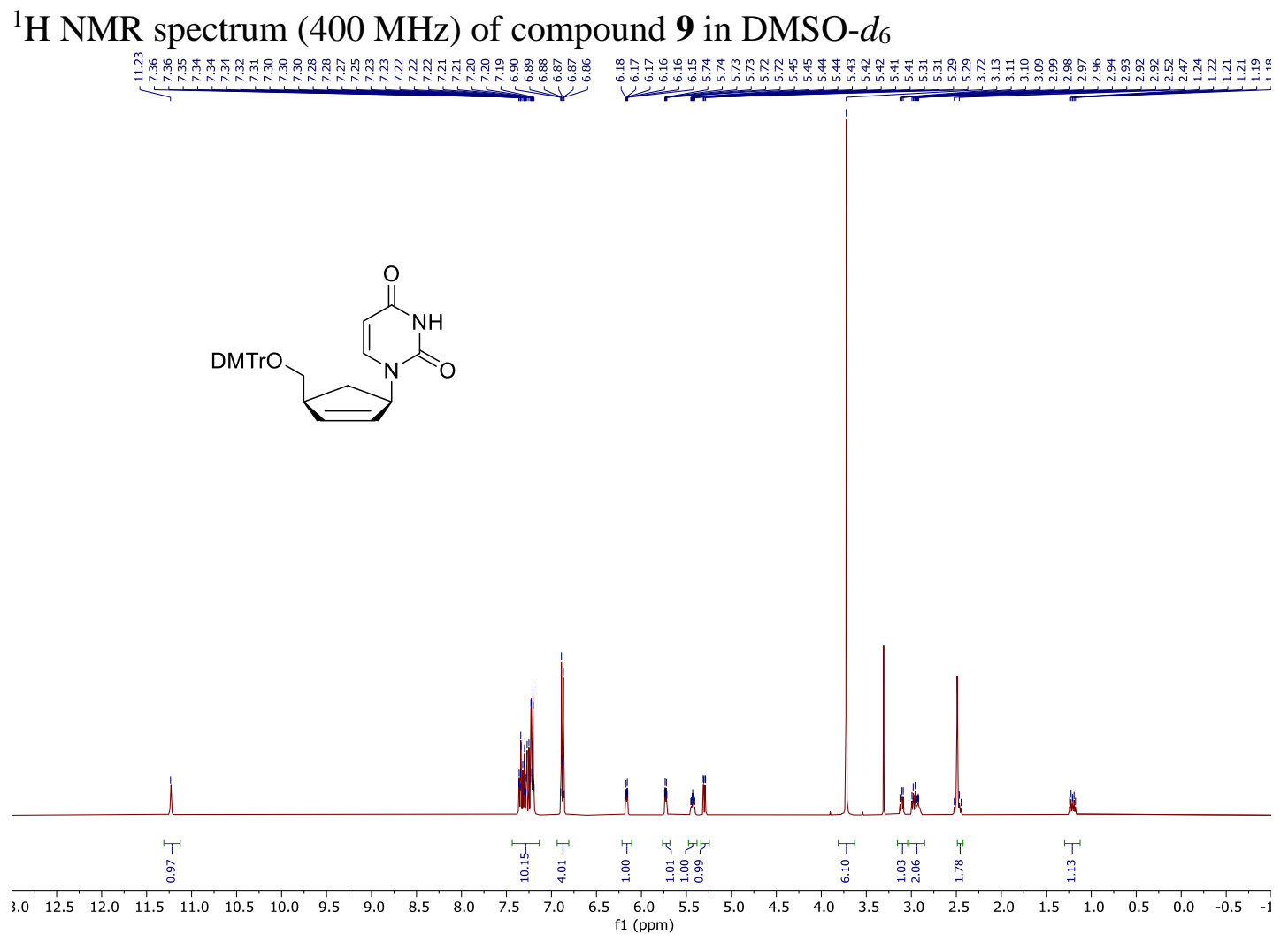

${ }^{13} \mathrm{C}$ NMR spectrum $(101 \mathrm{MHz})$ of compound 9 in DMSO- $d_{6}$
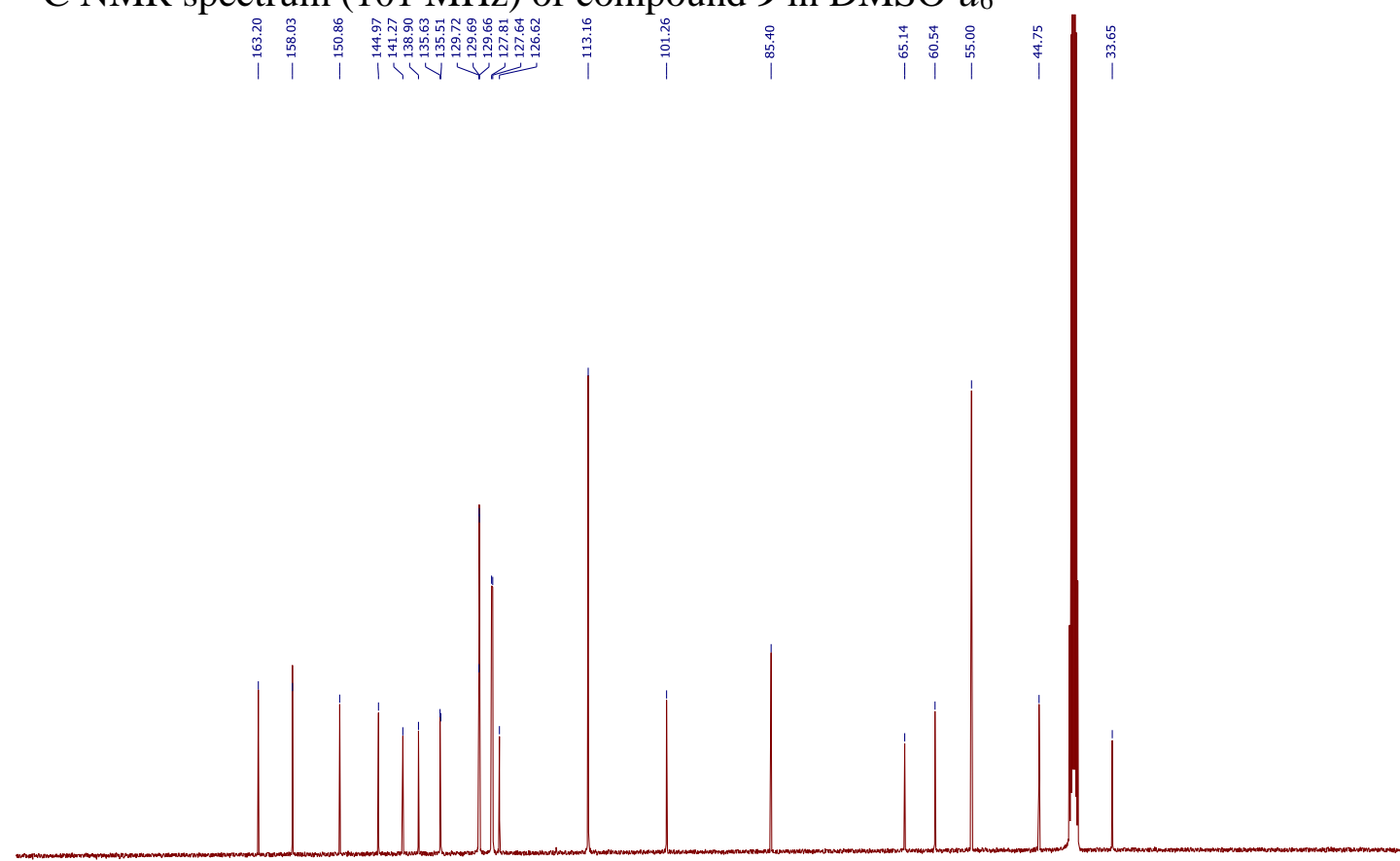

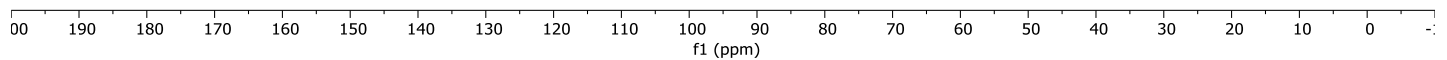




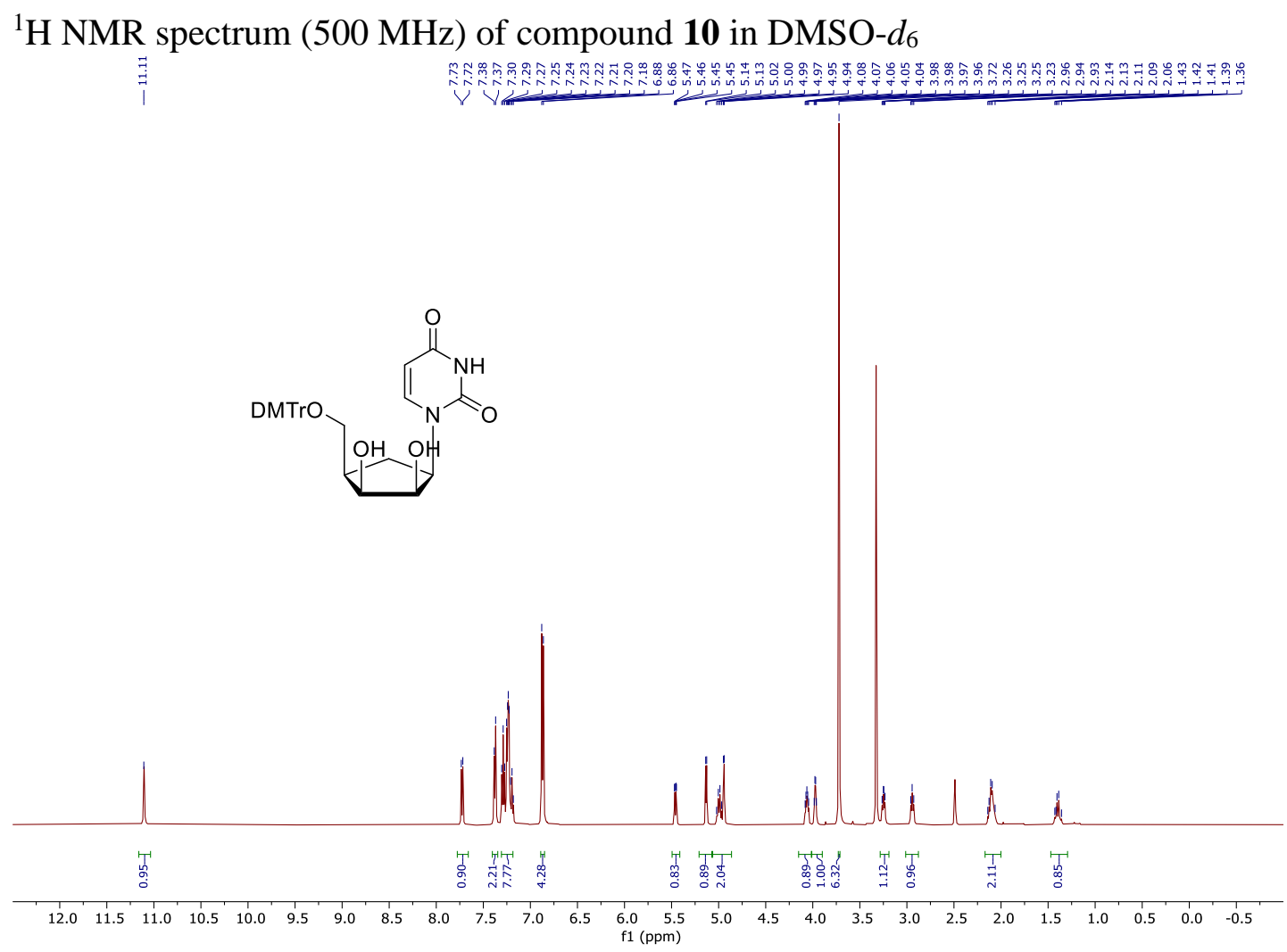

${ }^{13} \mathrm{C}$ NMR spectrum (126 MHz) of compound 10 in DMSO- $d_{6}$

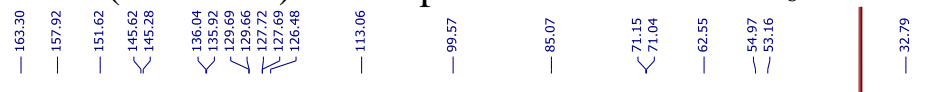

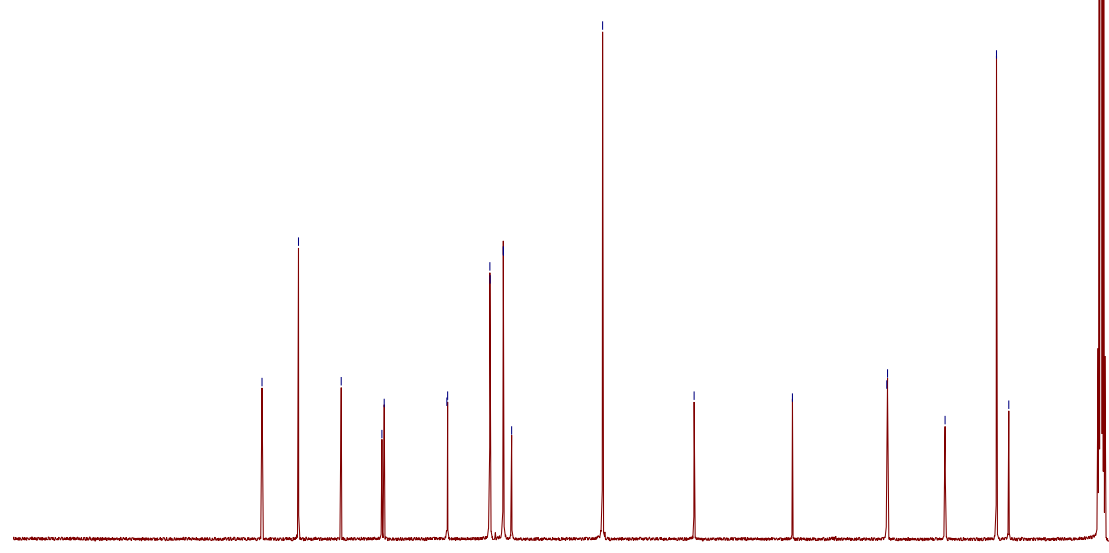

\begin{tabular}{llllllllllllllllllllll}
\hline 00 & 190 & 180 & 170 & 160 & 150 & 140 & 130 & 120 & 110 & $\underset{f}{1} 100$ & 90 & 80 & 70 & 60 & 50 & 40 & 30 & 20 & 10 & 0 & -
\end{tabular} 
${ }^{1} \mathrm{H}-{ }^{1} \mathrm{H}$ COSY spectrum of compound $\mathbf{1 0}$ in DMSO- $d_{6}$

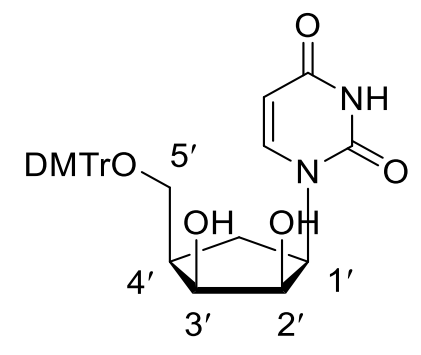

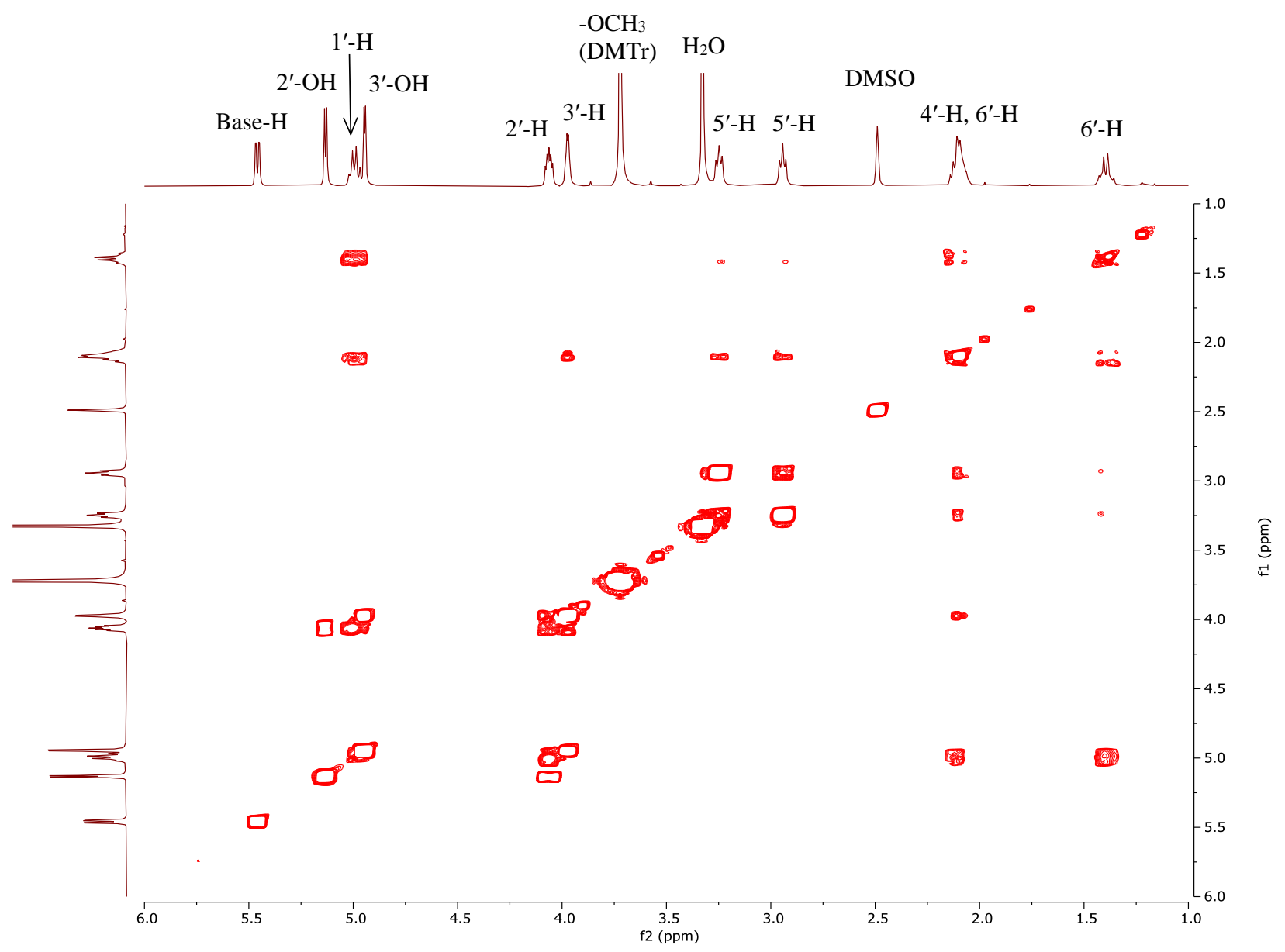


$2 \mathrm{D}{ }^{1} \mathrm{H}-{ }^{1} \mathrm{H}$ NOESY spectrum of compound 10 in DMSO- $d_{6}$

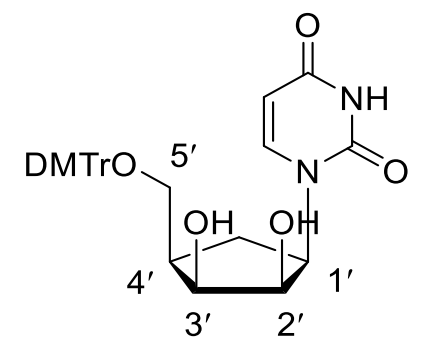

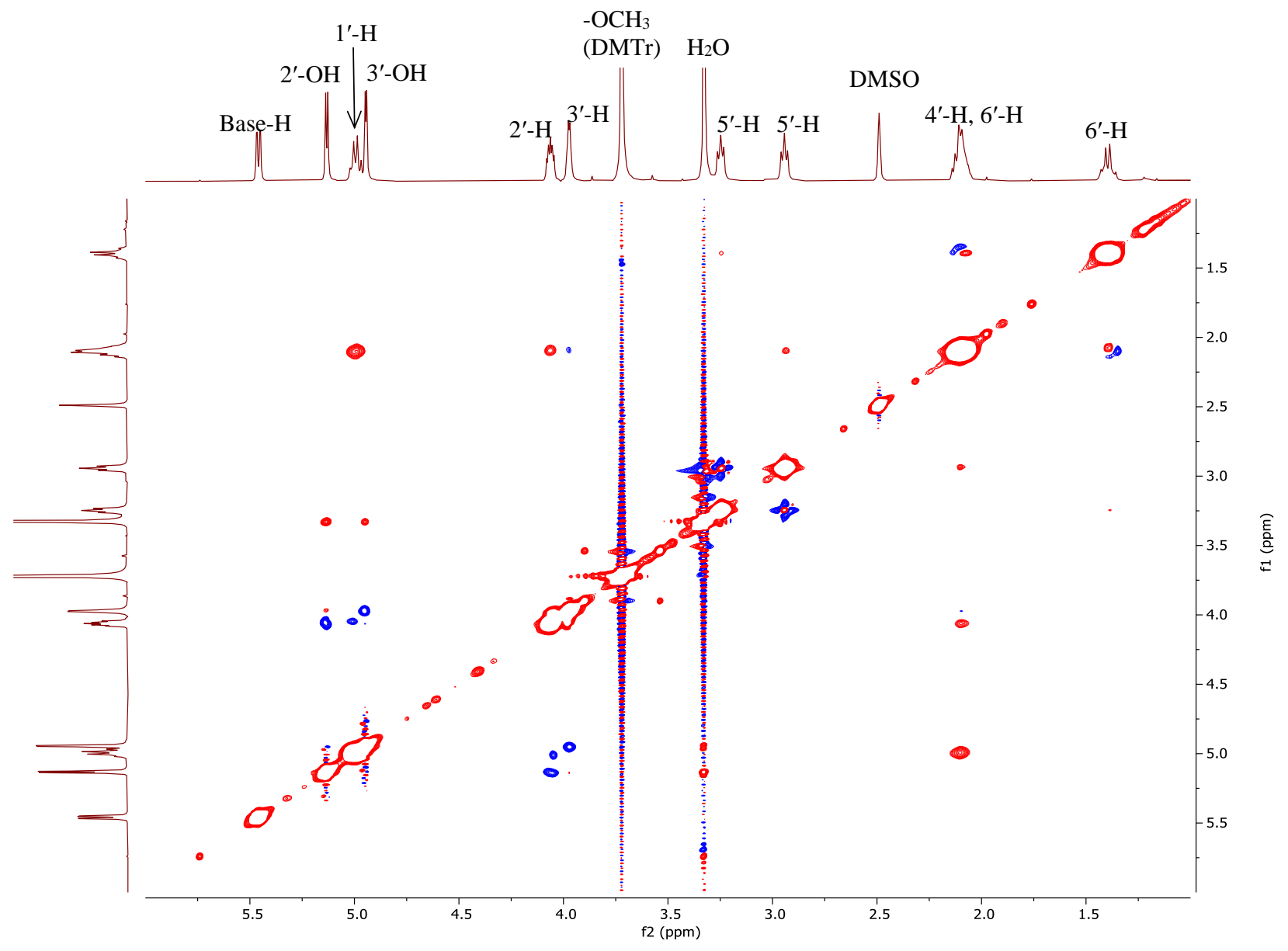




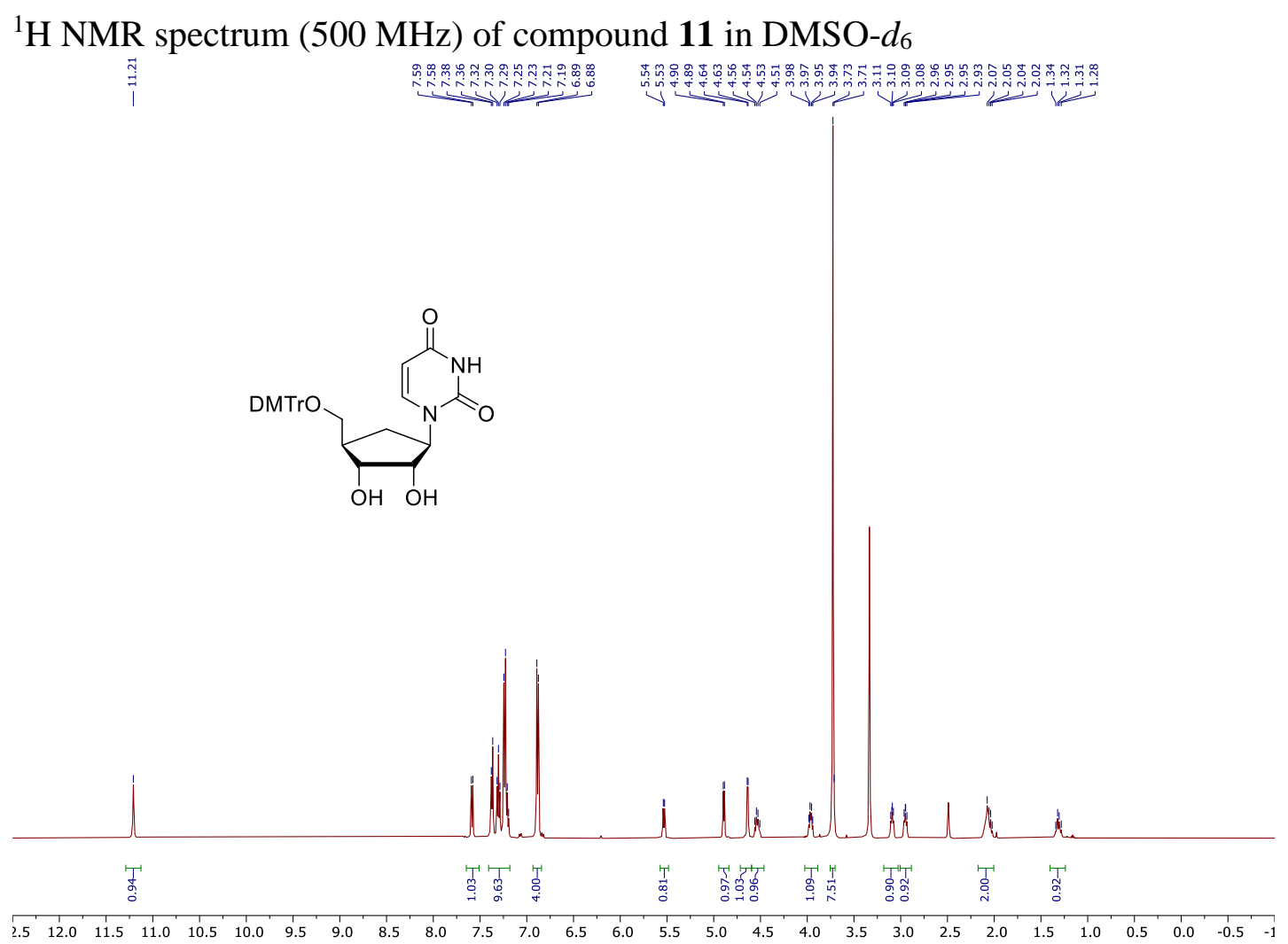

${ }^{13} \mathrm{C}$ NMR spectrum $(126 \mathrm{MHz})$ of compound 11 in DMSO-d
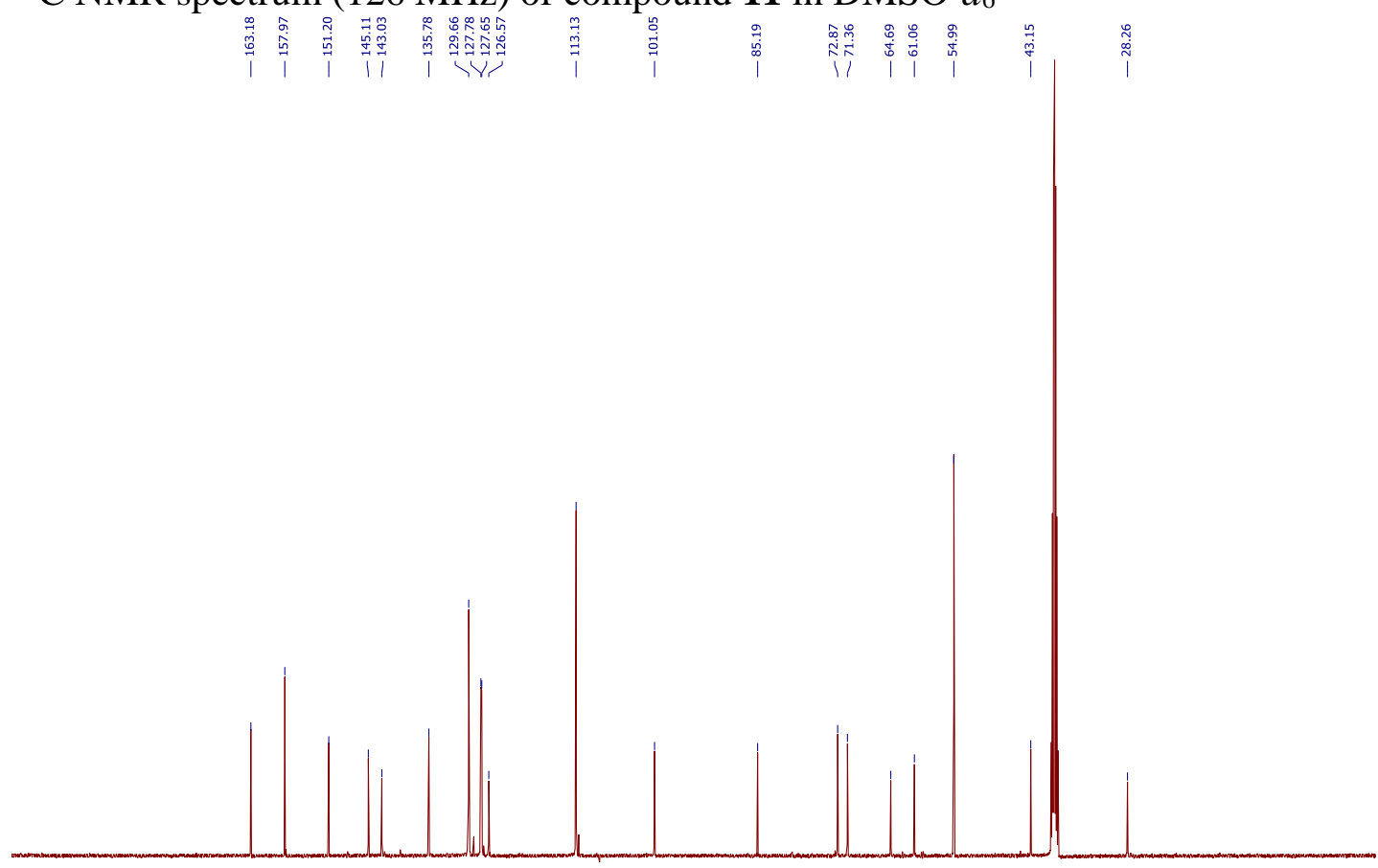

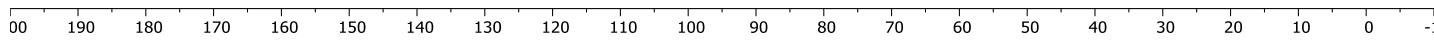


${ }^{1} \mathrm{H}-{ }^{1} \mathrm{H}$ COSY spectrum of compound $\mathbf{1 1}$ in DMSO- $d_{6}$

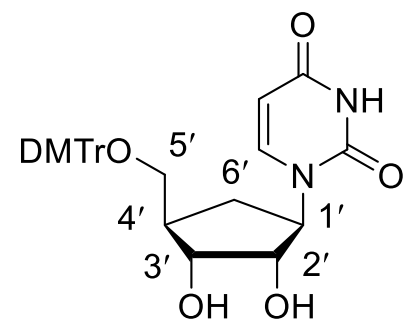

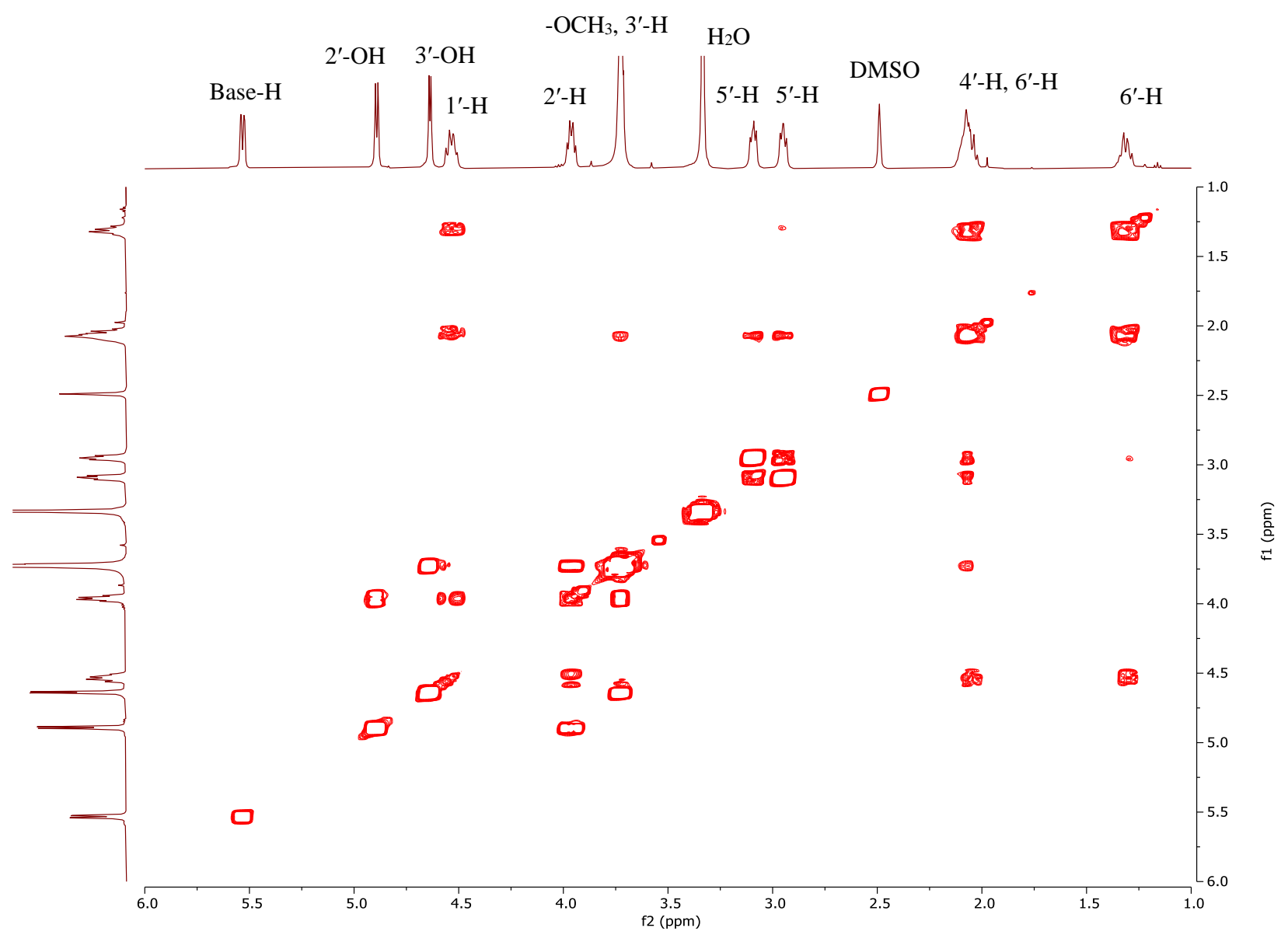


$2 \mathrm{D}^{1} \mathrm{H}-{ }^{1} \mathrm{H}$ NOESY spectrum of compound $\mathbf{1 1}$ in DMSO- $d_{6}$

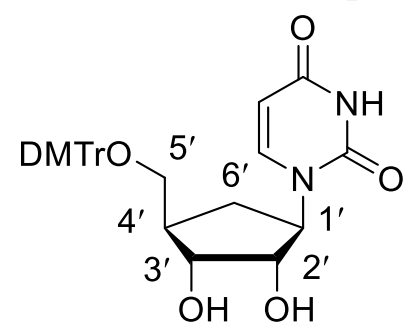

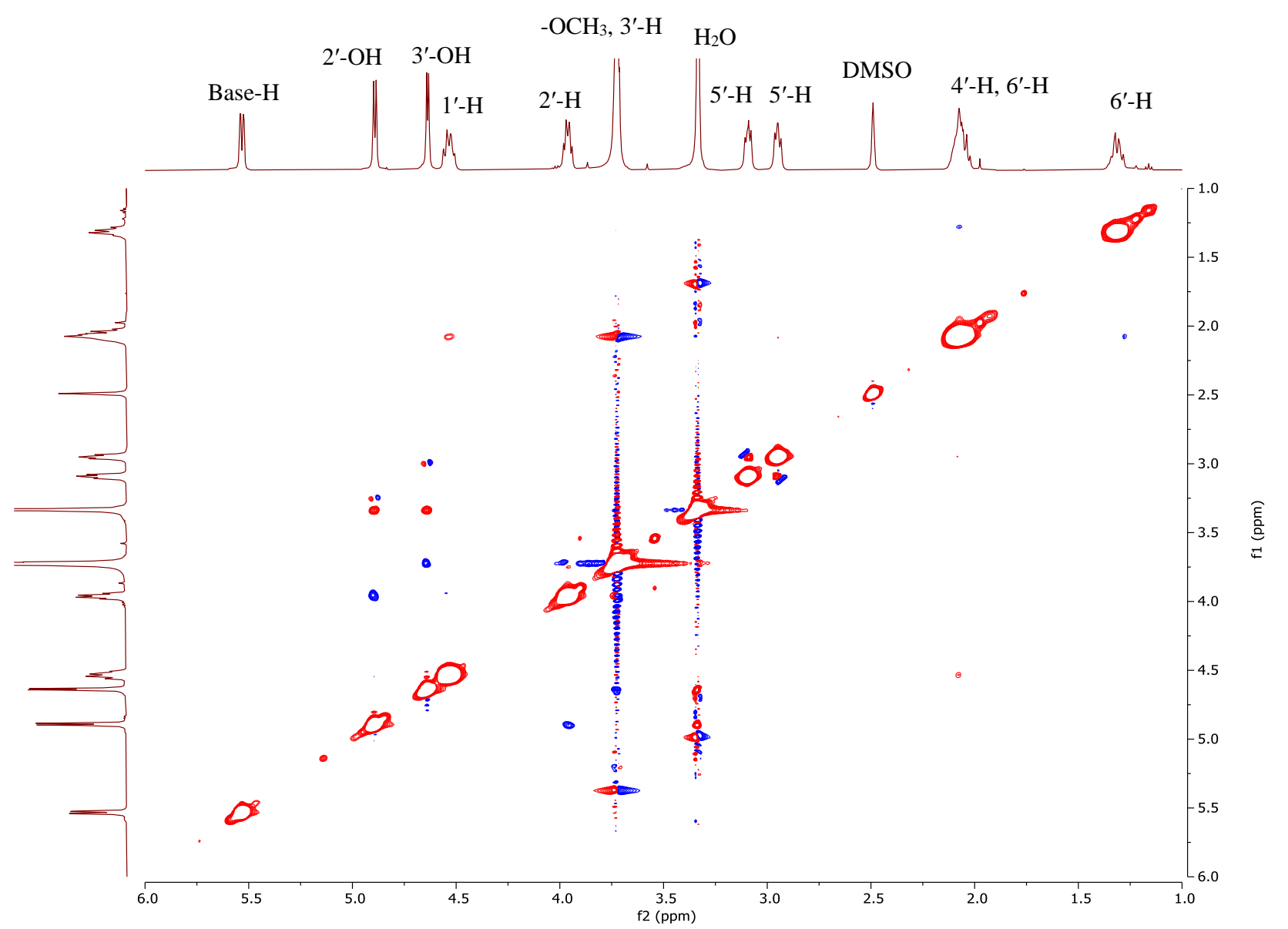




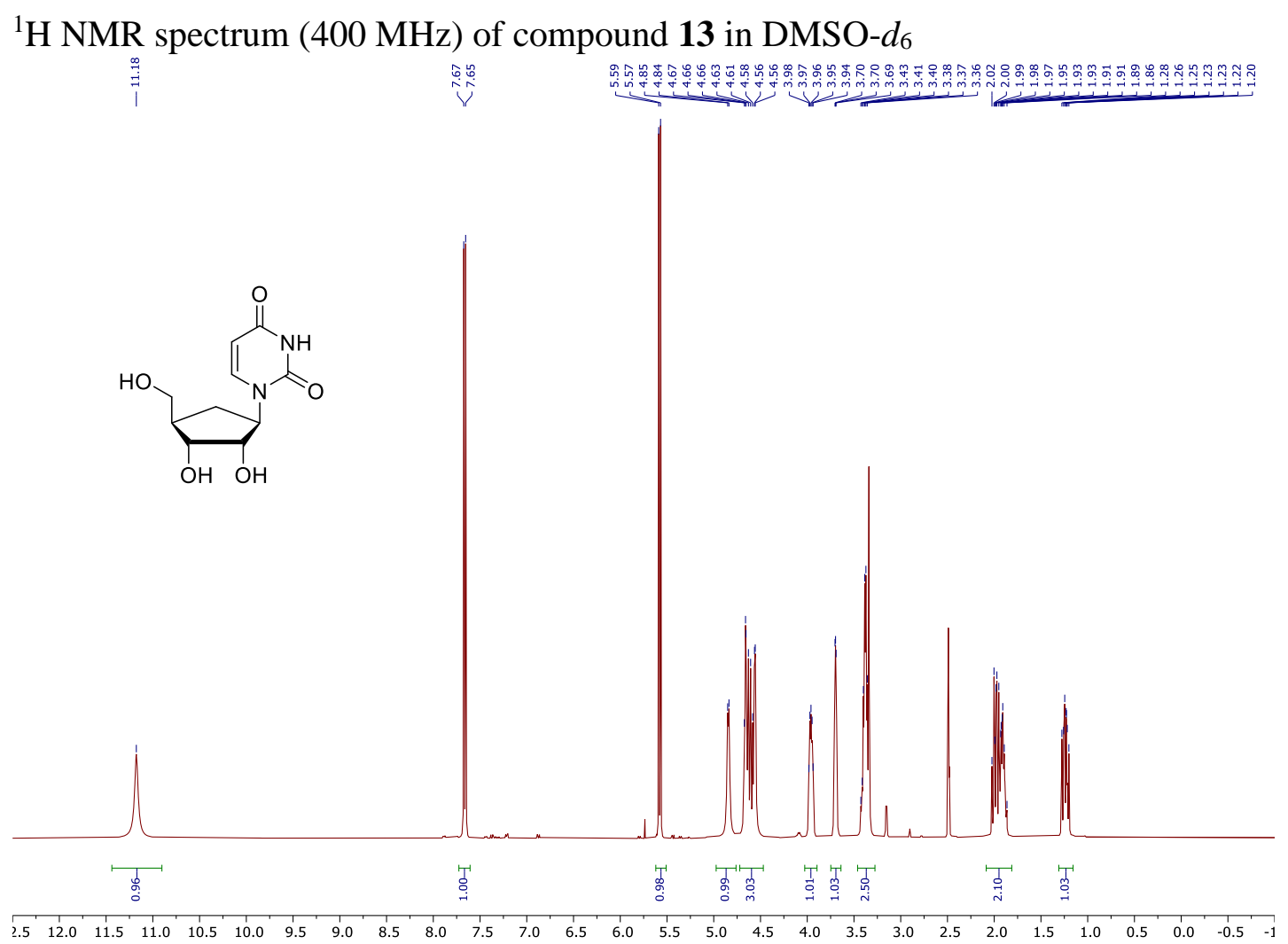

${ }^{13} \mathrm{C}$ NMR spectrum (126 MHz) of compound 13 in DMSO-d

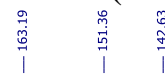

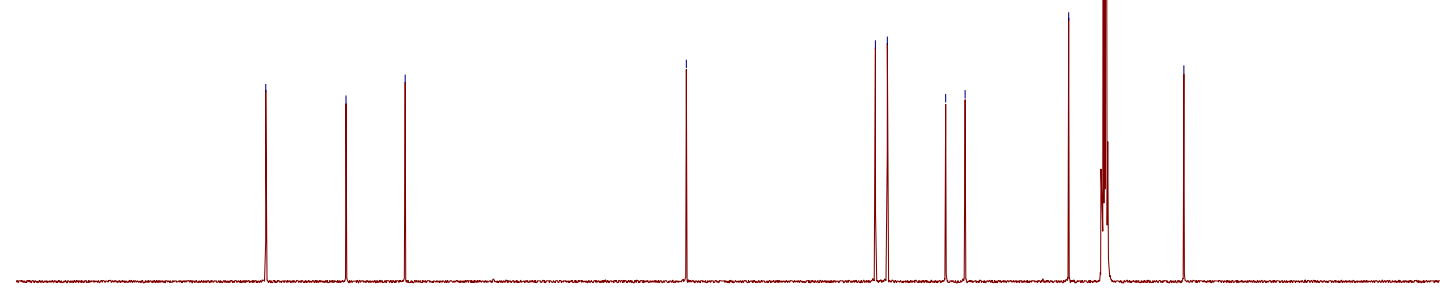

\begin{tabular}{llllllllllllllllllllll}
\hline & 190 & 180 & 170 & 160 & 150 & 140 & 130 & 120 & 110 & 100 & 90 & 80 & 70 & 60 & 50 & 40 & 30 & 20 & 10 & 0 & -1
\end{tabular} 
${ }^{1} \mathrm{H}$ NMR spectrum $(400 \mathrm{MHz})$ of compound 14 in DMSO- $d_{6}$

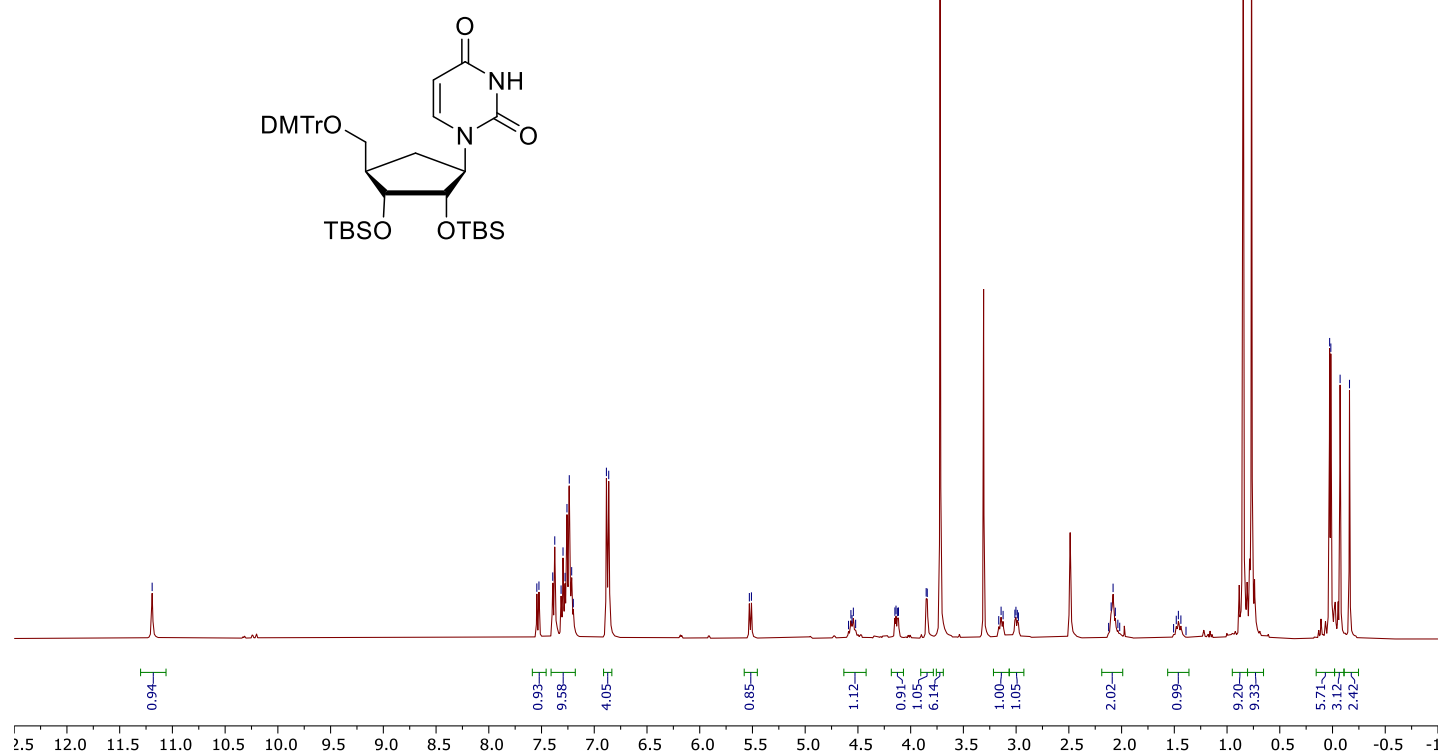

年

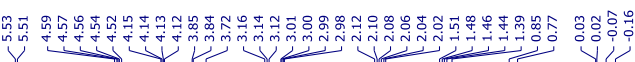

${ }^{13} \mathrm{C}$ NMR spectrum $(101 \mathrm{MHz})$ of compound 14 in DMSO- $d_{6}$
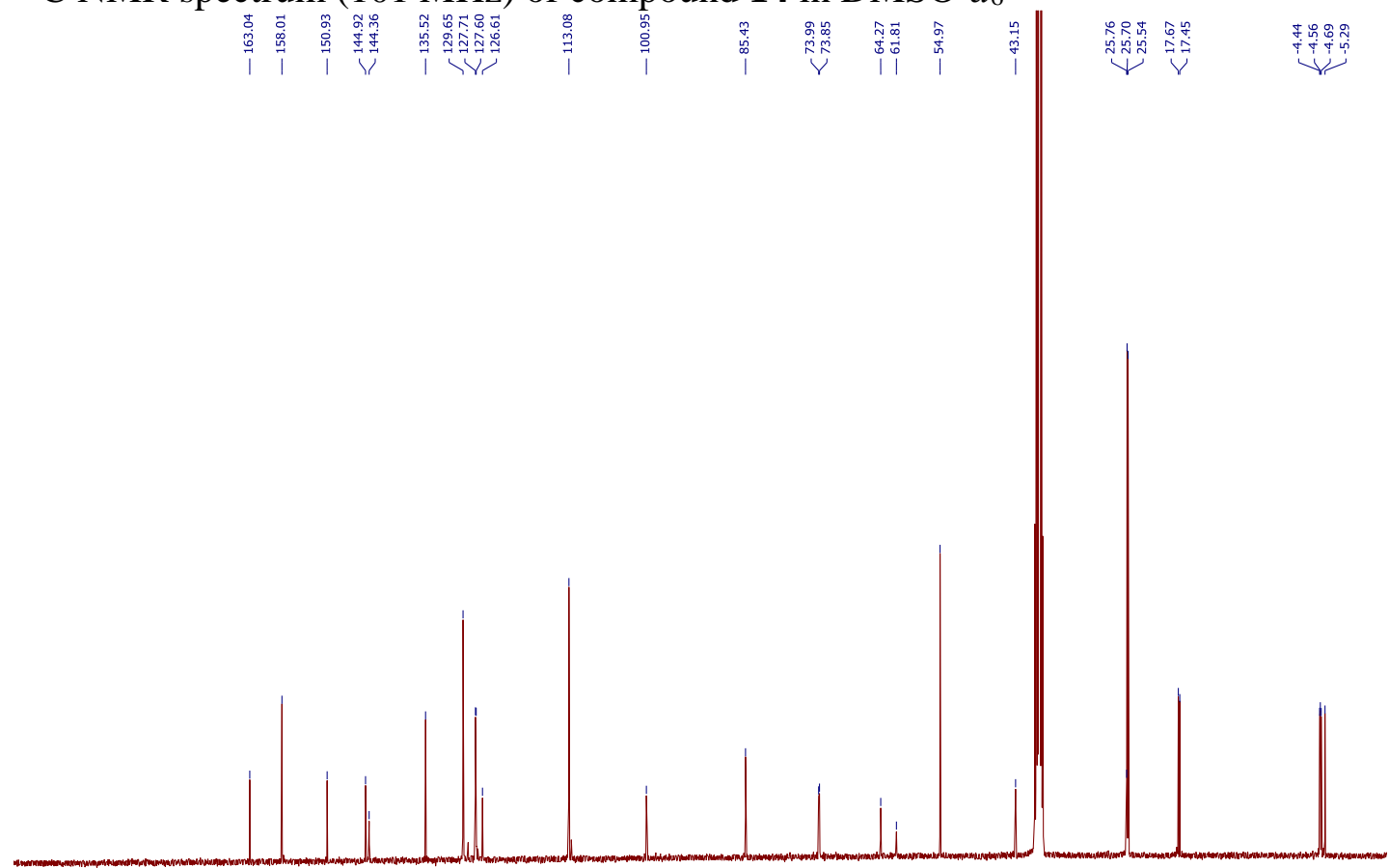

00 


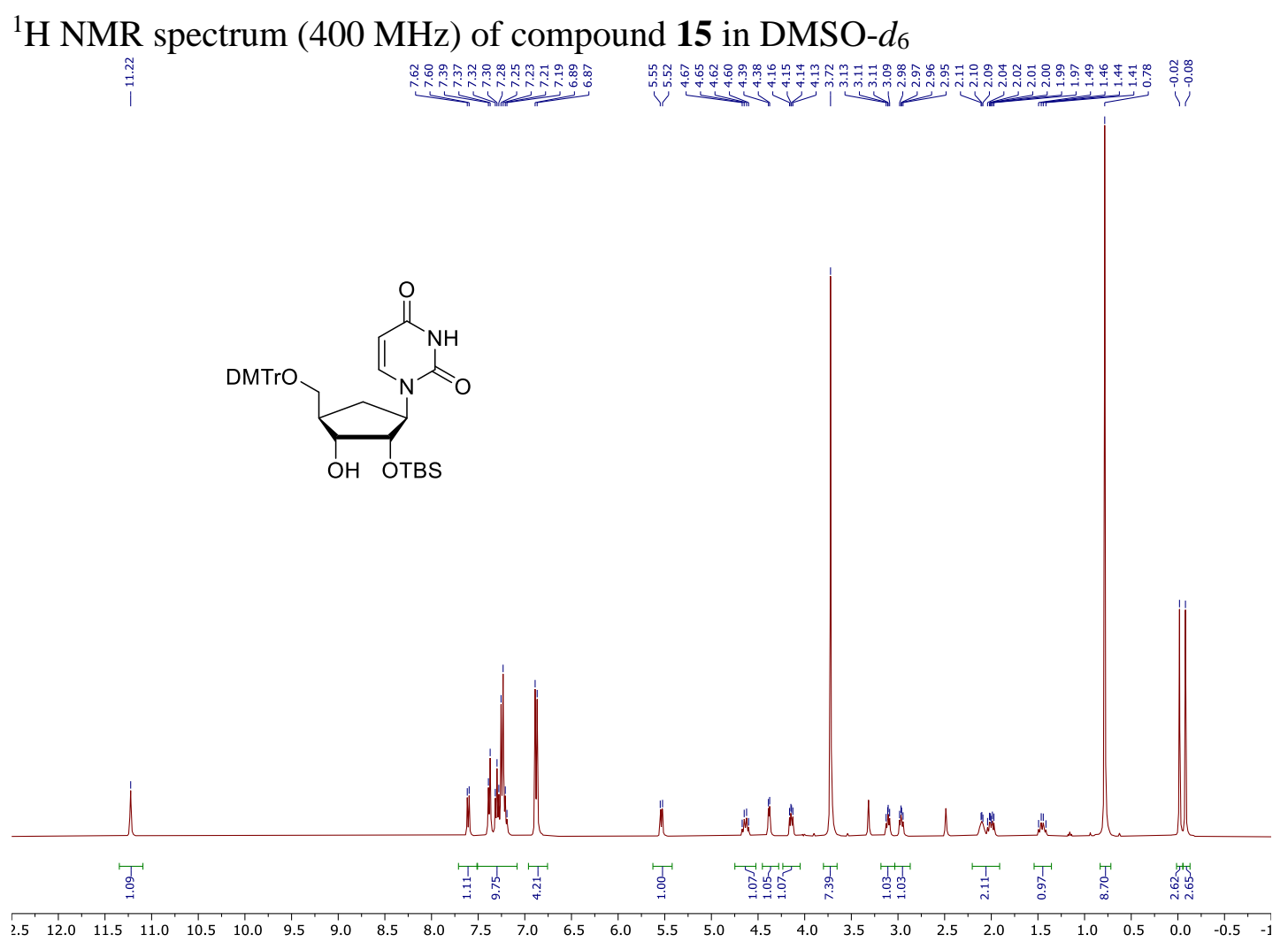

${ }^{13} \mathrm{C}$ NMR spectrum (126 MHz) of compound 15 in DMSO- $d_{6}$

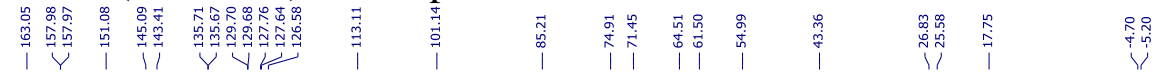

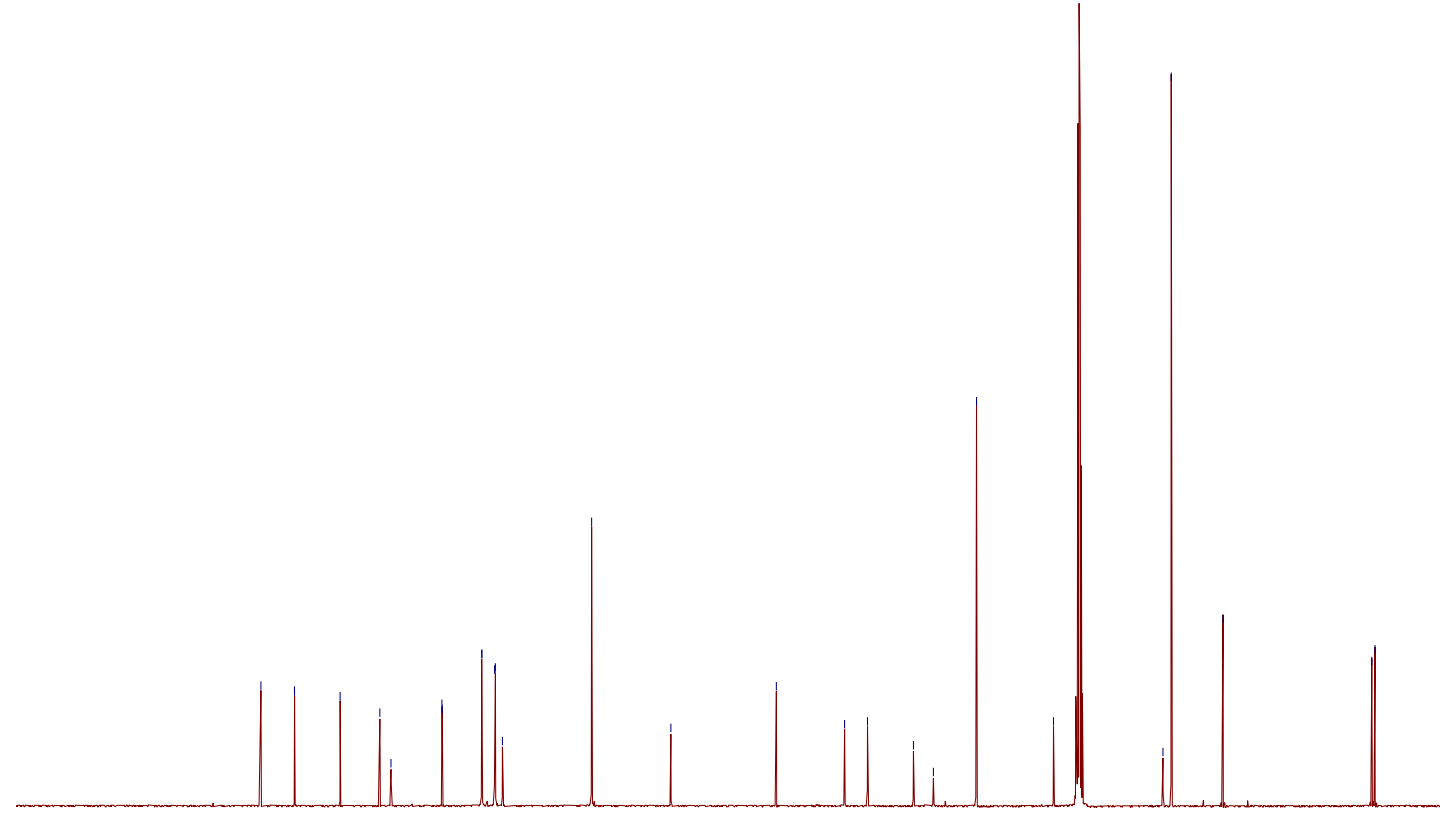

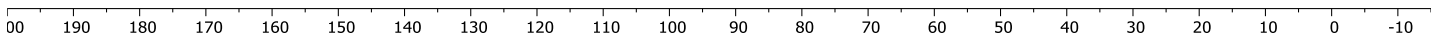


${ }^{1} \mathrm{H}-{ }^{1} \mathrm{H}$ COSY spectrum of compound $\mathbf{1 5}$ in DMSO- $d_{6}$
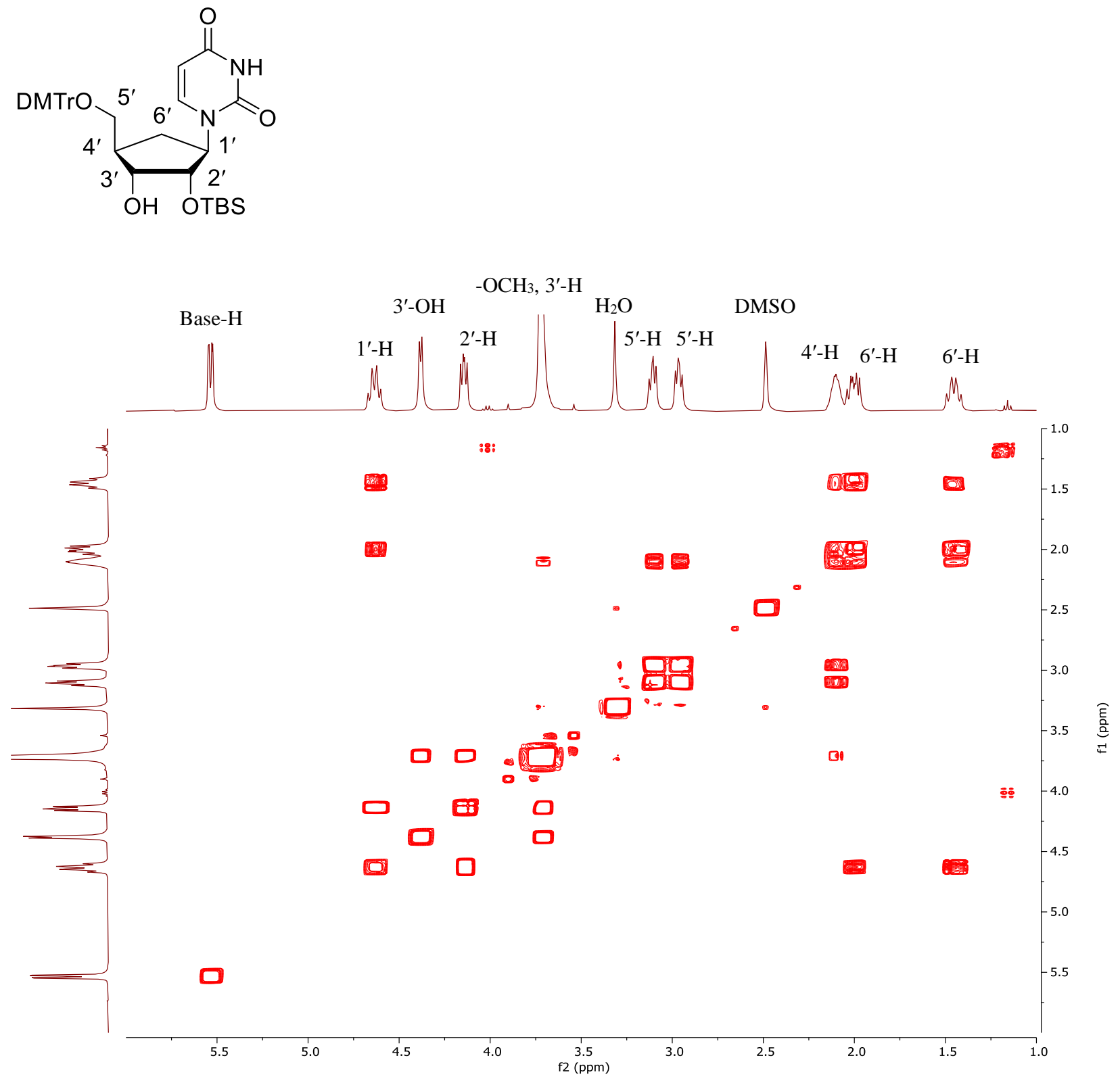
${ }^{1} \mathrm{H}$ NMR spectrum $(400 \mathrm{MHz})$ of compound 16 in DMSO- $d_{6}$

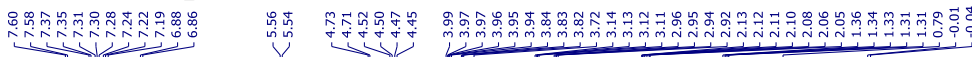
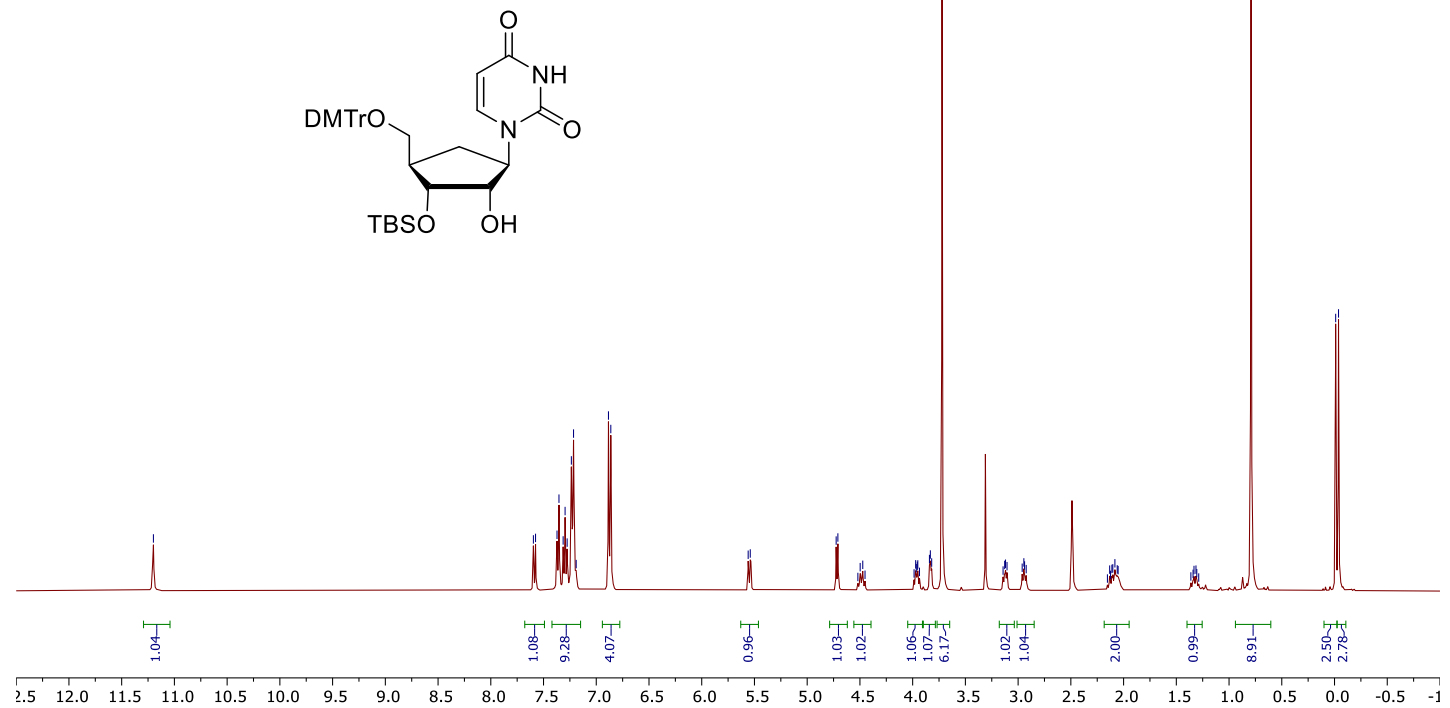

${ }^{13} \mathrm{C}$ NMR spectrum $(101 \mathrm{MHz})$ of compound 16 in DMSO- $d_{6}$

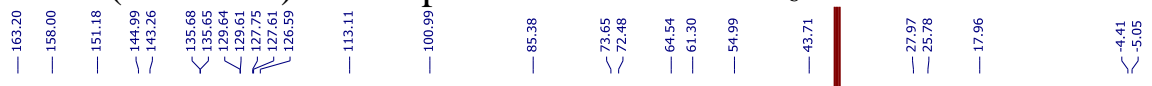

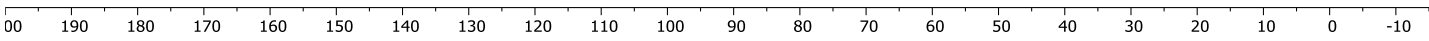


${ }^{1} \mathrm{H}-{ }^{1} \mathrm{H}$ COSY spectrum of compound $\mathbf{1 6}$ in DMSO- $d_{6}$
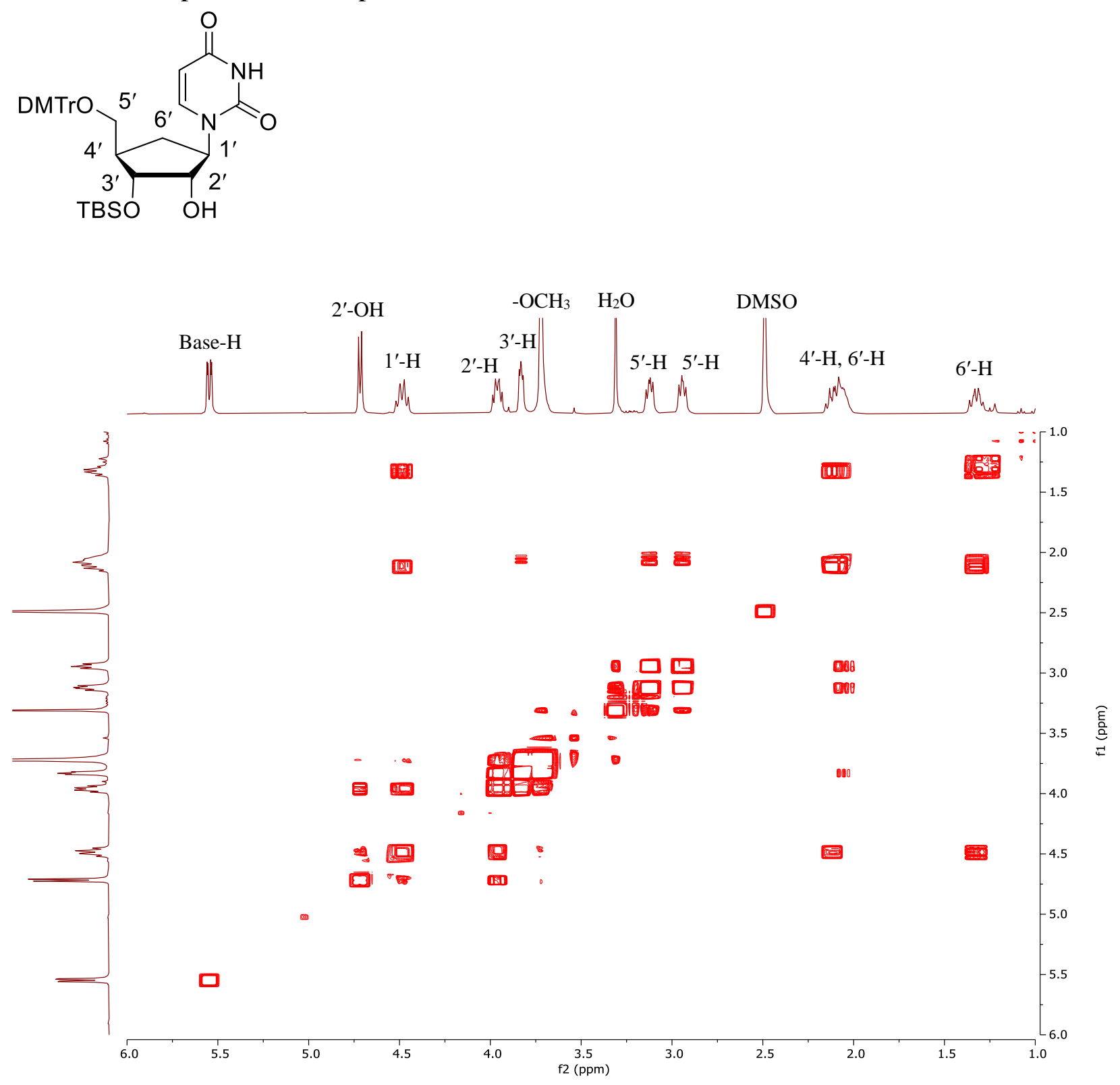
${ }^{1} \mathrm{H}$ NMR spectrum $(500 \mathrm{MHz})$ of compound $\mathbf{1}$ in $\mathrm{CD}_{3} \mathrm{CN}$

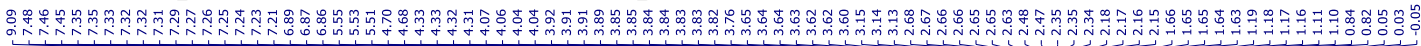
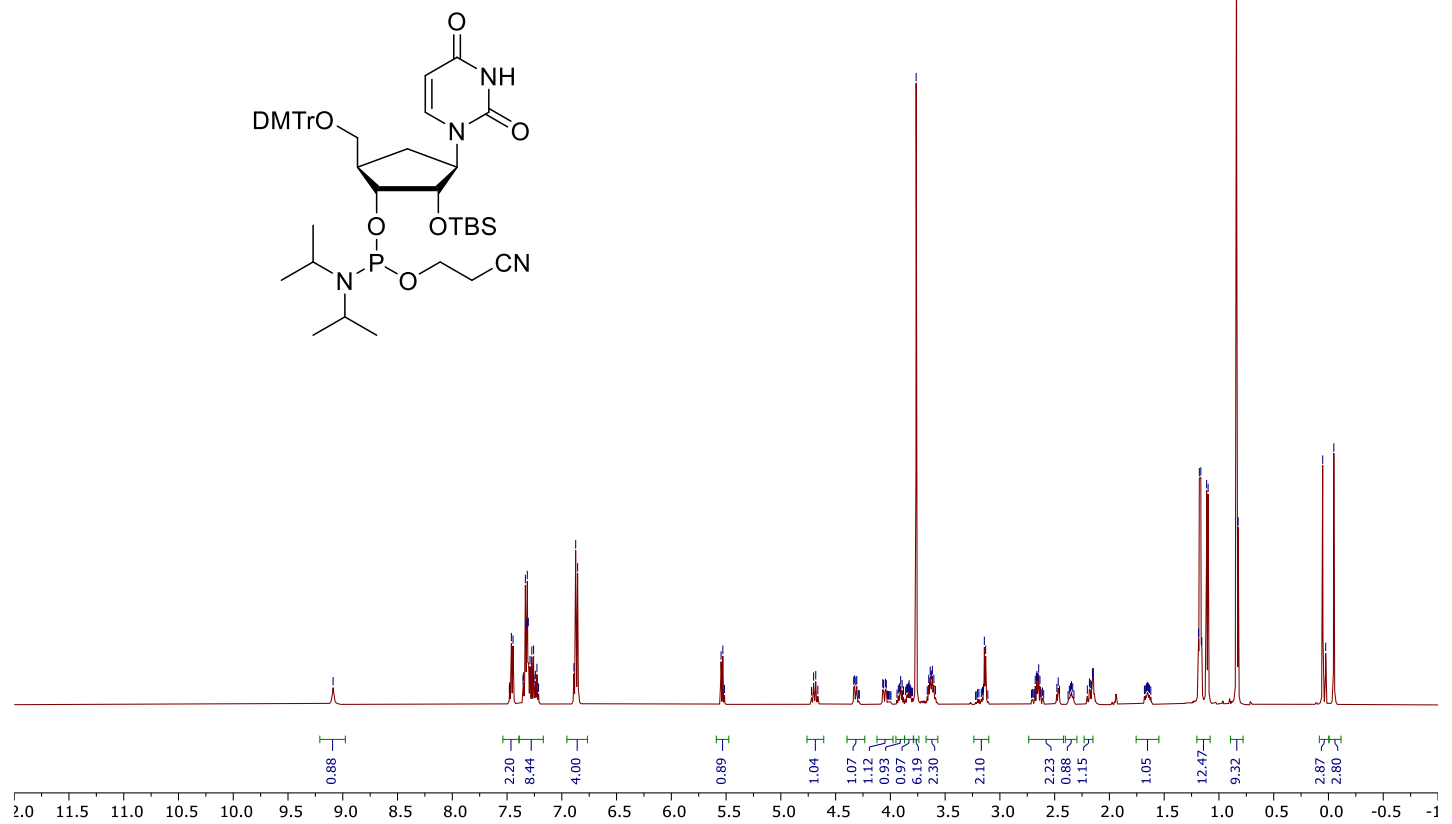

${ }^{13} \mathrm{C}$ NMR spectrum (101 MHz) of compound $\mathbf{1}$ in $\mathrm{CD}_{3} \mathrm{CN}$

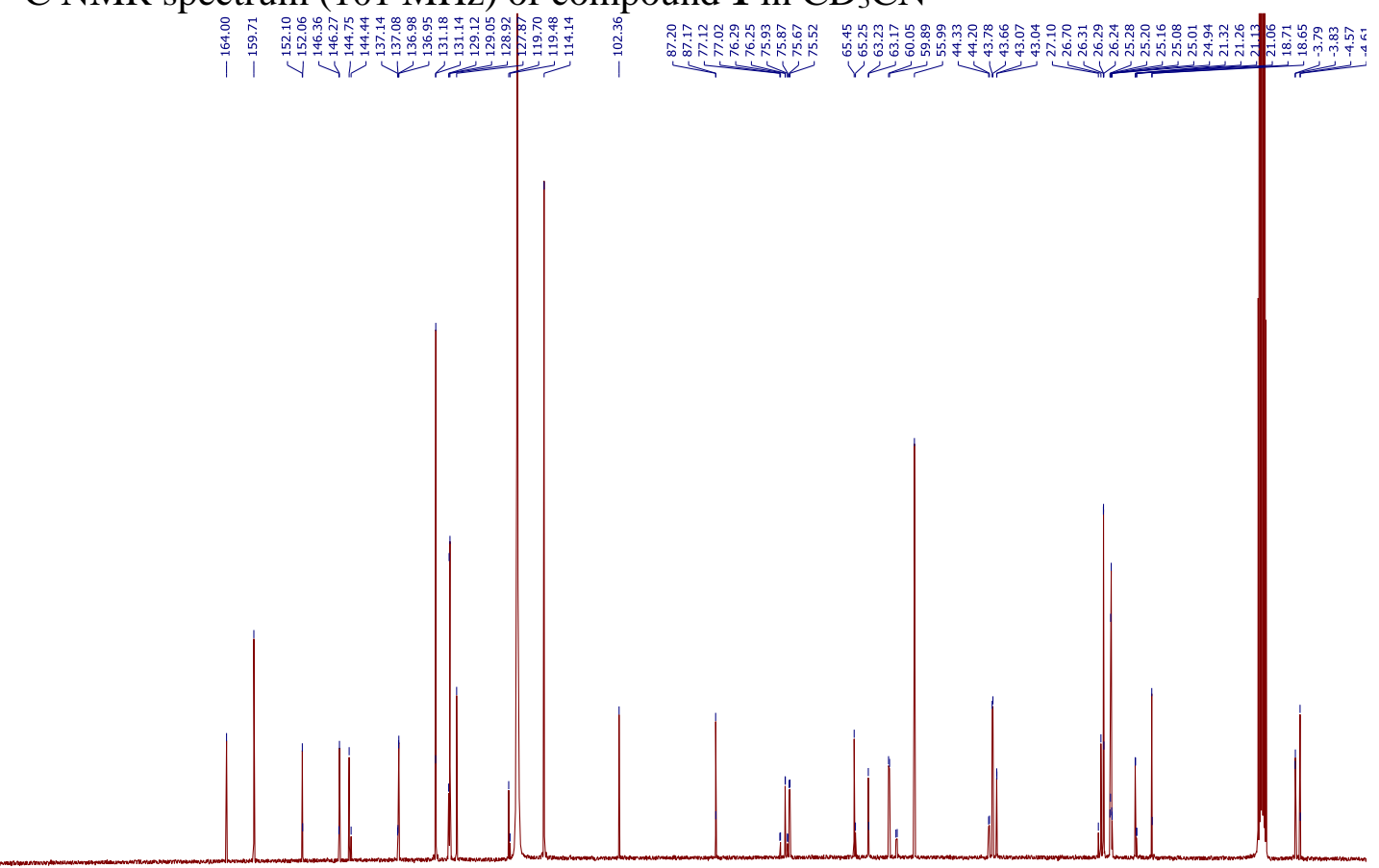

00 
${ }^{31} \mathrm{P}$ NMR spectrum $(162 \mathrm{MHz})$ of compound $\mathbf{1}$ in $\mathrm{CD}_{3} \mathrm{CN}$

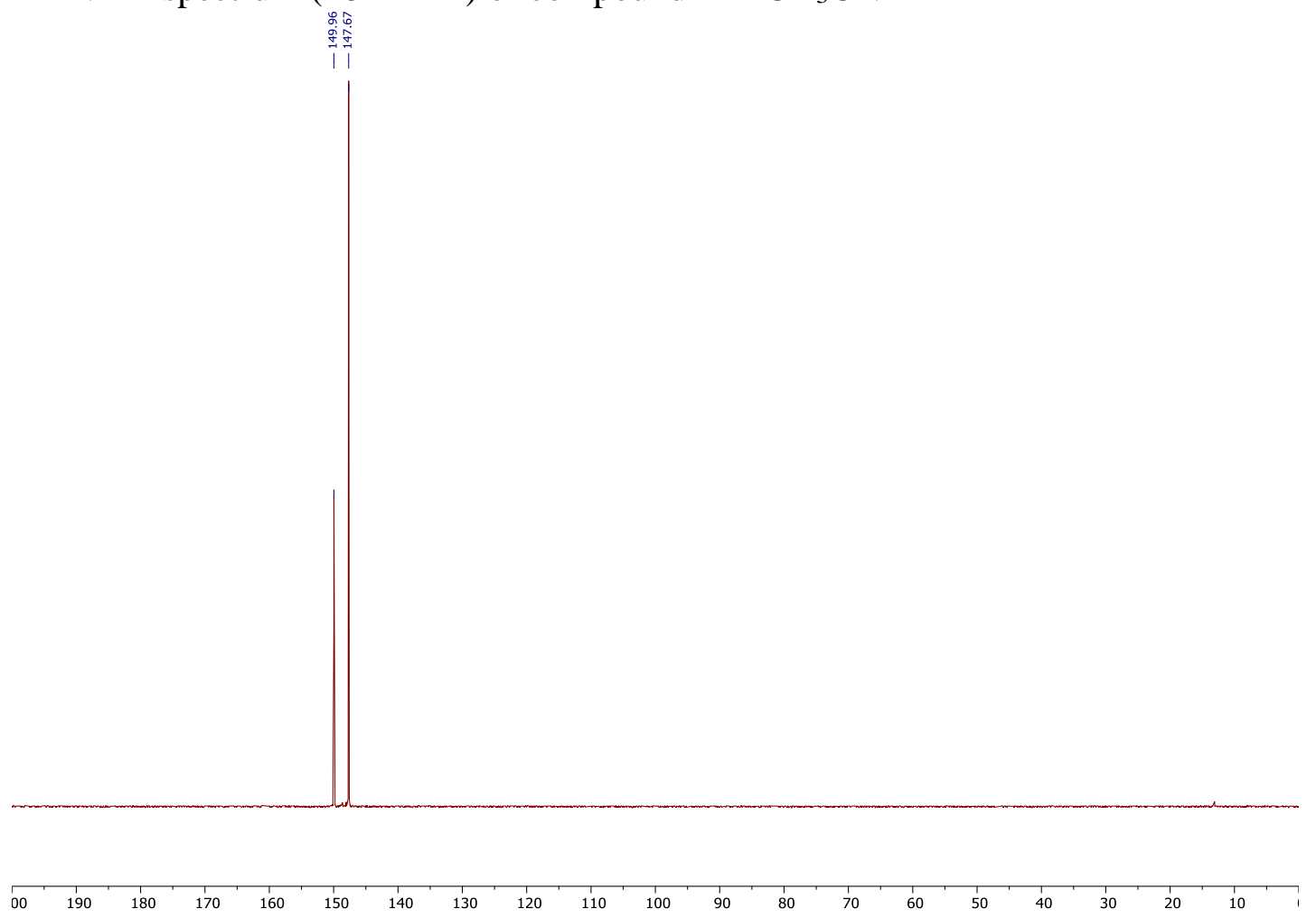


${ }^{1} \mathrm{H}$ NMR spectrum (400 MHz) of compound 2 in $\mathrm{CD}_{3} \mathrm{CN}$

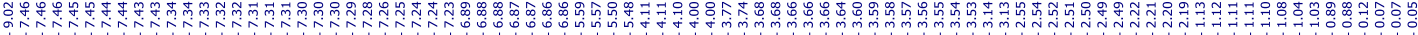

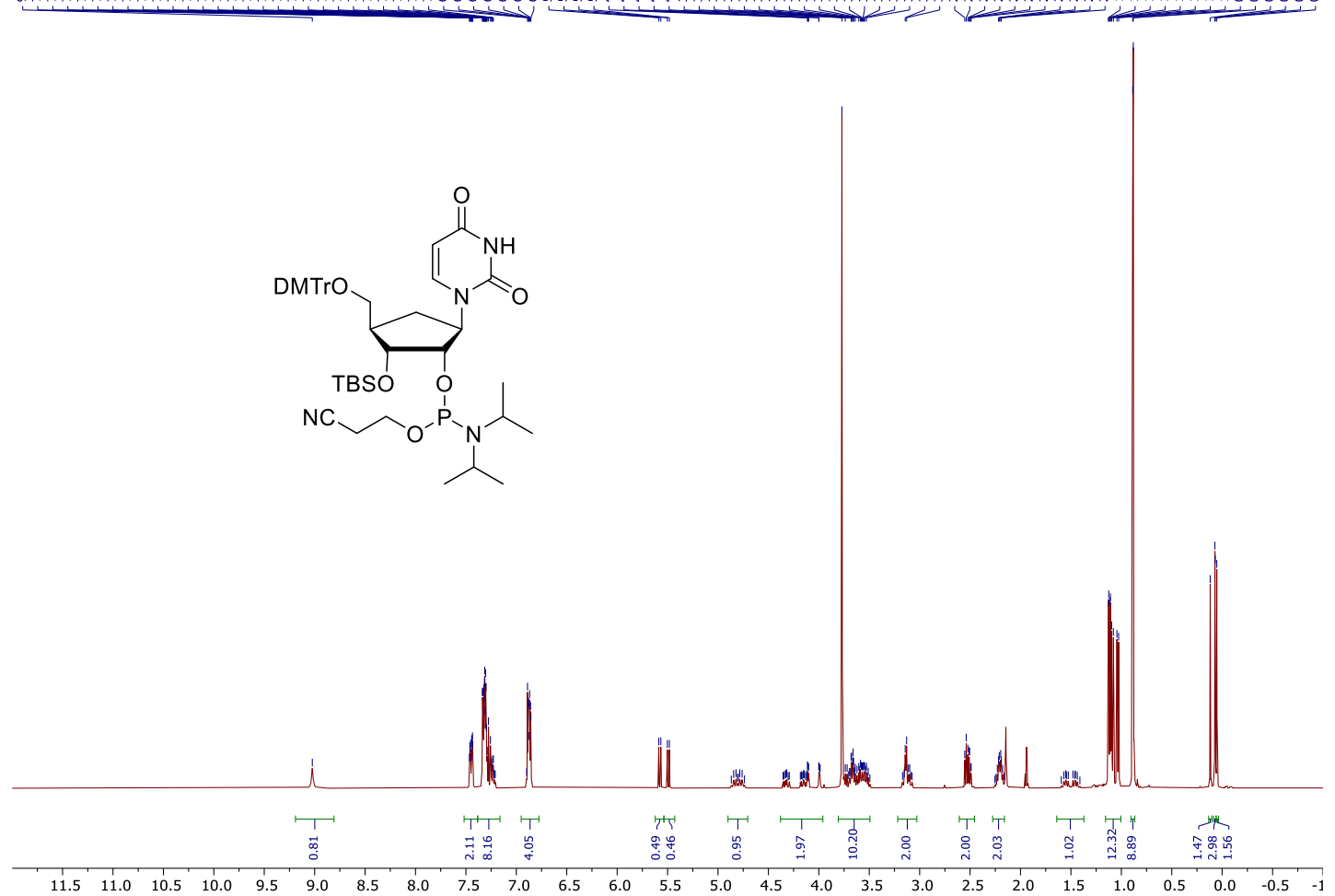

${ }^{13} \mathrm{C}$ NMR spectrum (101 MHz) of compound 2 in $\mathrm{CD}_{3} \mathrm{CN}$

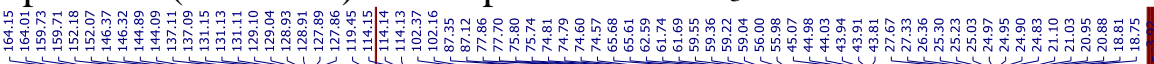

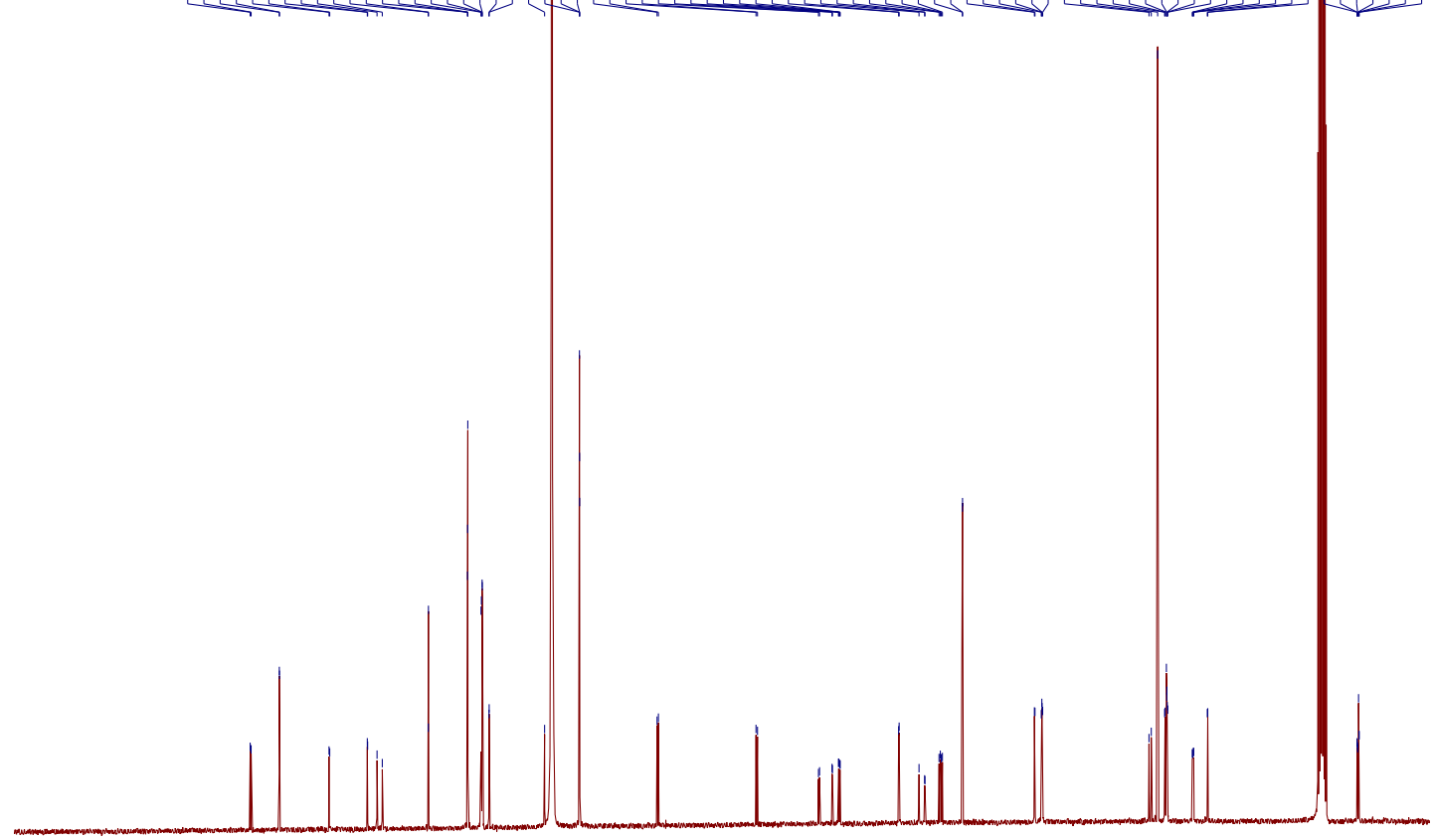

00

$\begin{array}{rrrrrr}190 & 180 & 170 & 160 & 150 & 140\end{array}$

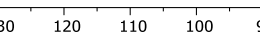
$\begin{array}{lllllll}50 & 40 & 30 & 20 & 10 & 0 & -10\end{array}$ 
${ }^{31} \mathrm{P}$ NMR spectrum $(202 \mathrm{MHz})$ of compound 2 in $\mathrm{CD}_{3} \mathrm{CN}$

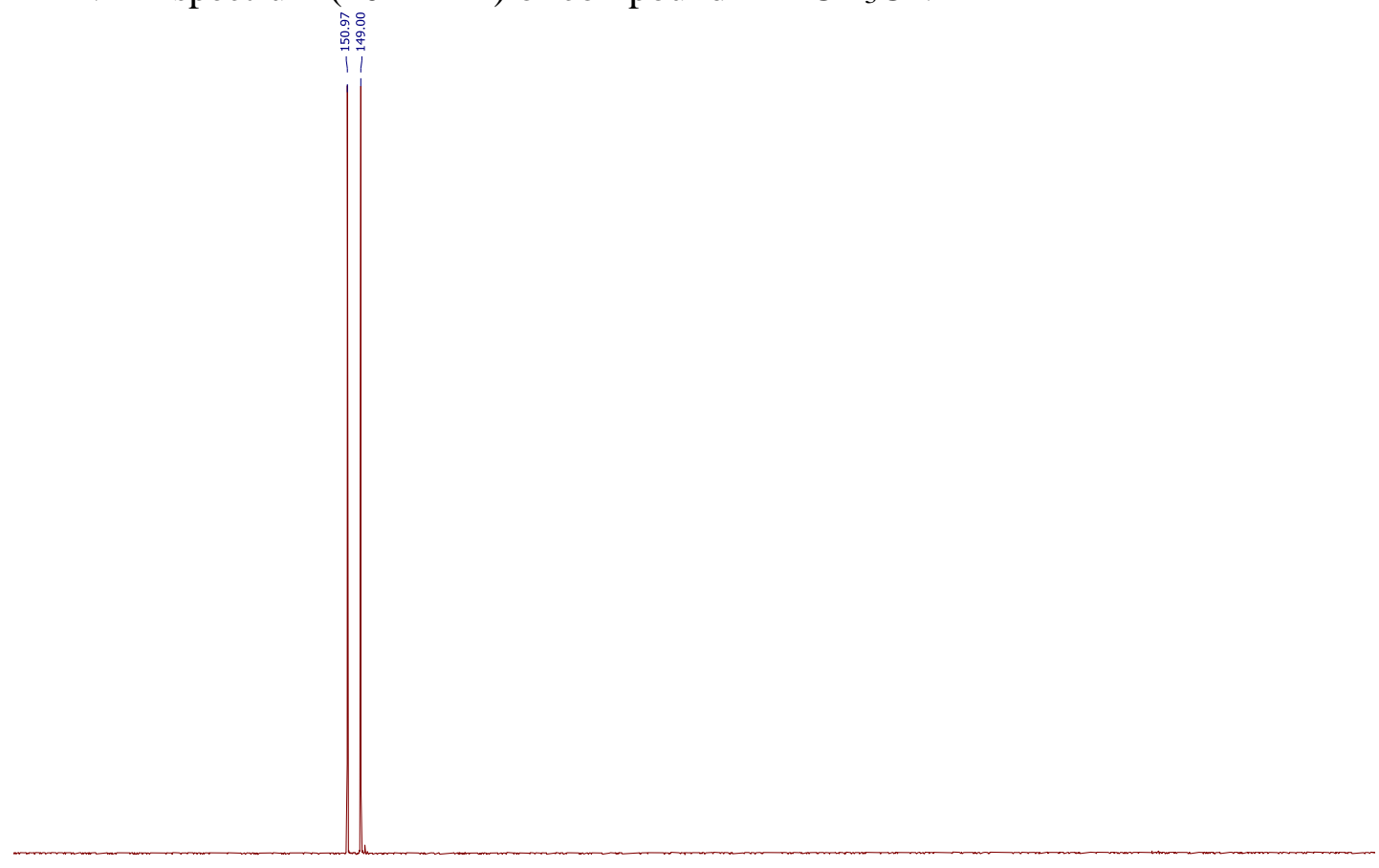

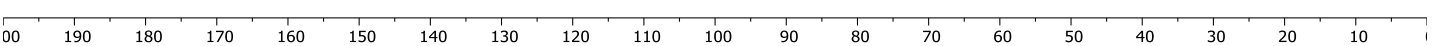


${ }^{1} \mathrm{H}$ NMR spectrum $(400 \mathrm{MHz})$ of compound 19 in DMSO- $d_{6}$

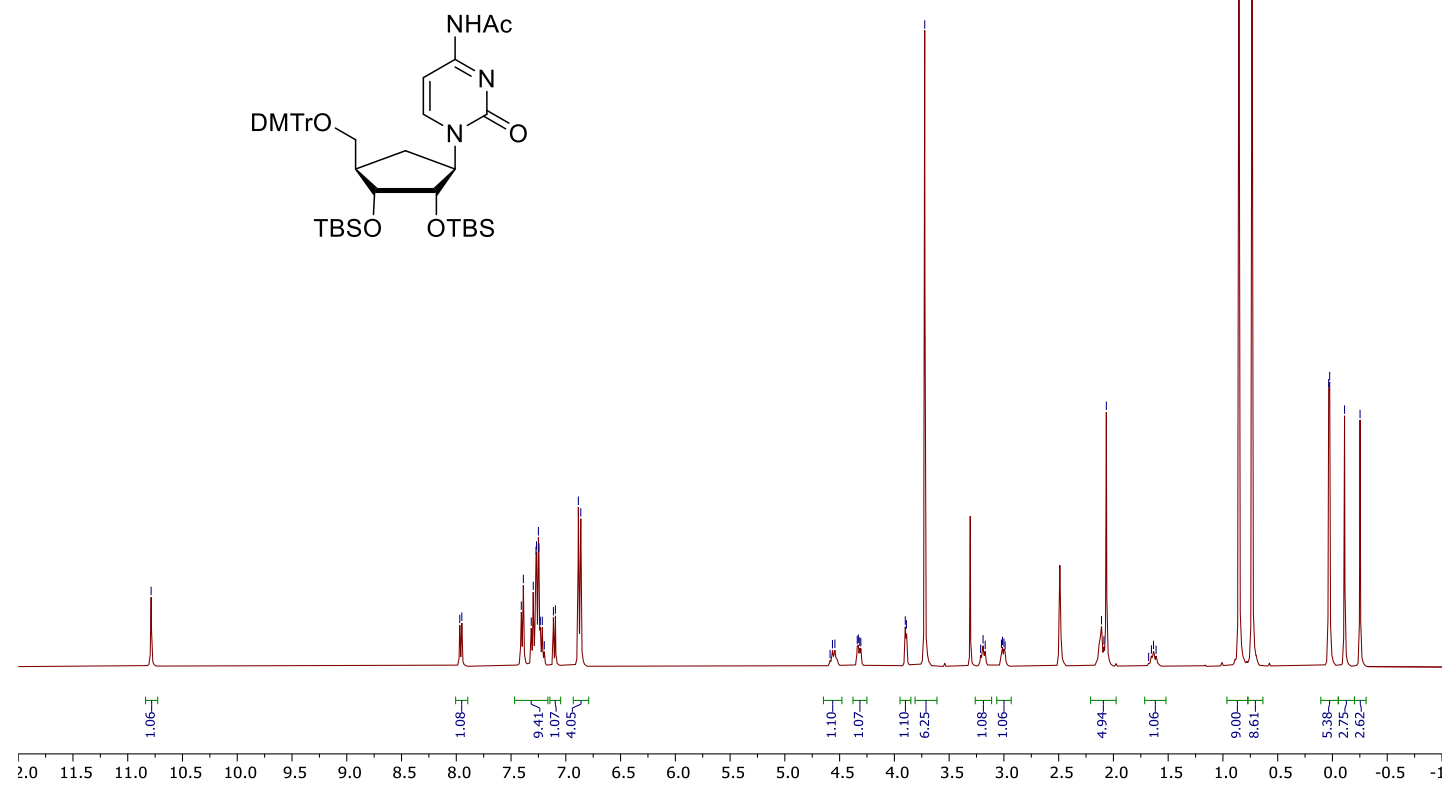

${ }^{13} \mathrm{C}$ NMR spectrum (101 MHz) of compound 19 in DMSO- $d_{6}$

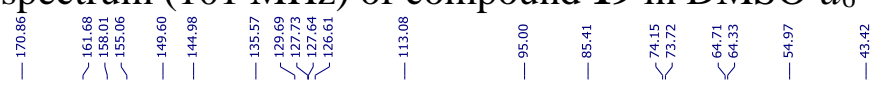
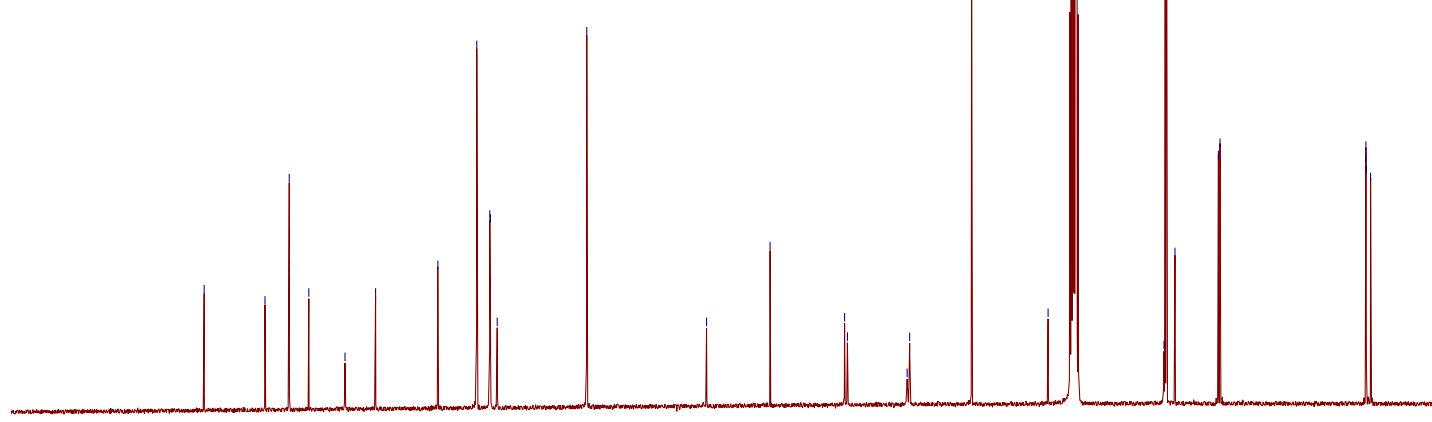

00

$\begin{array}{ccccccc}190 & 180 & 170 & 160 & 150 & 140 & 130\end{array}$ $\begin{array}{rrrr}120 & 110 & 100 & 90\end{array}$ 


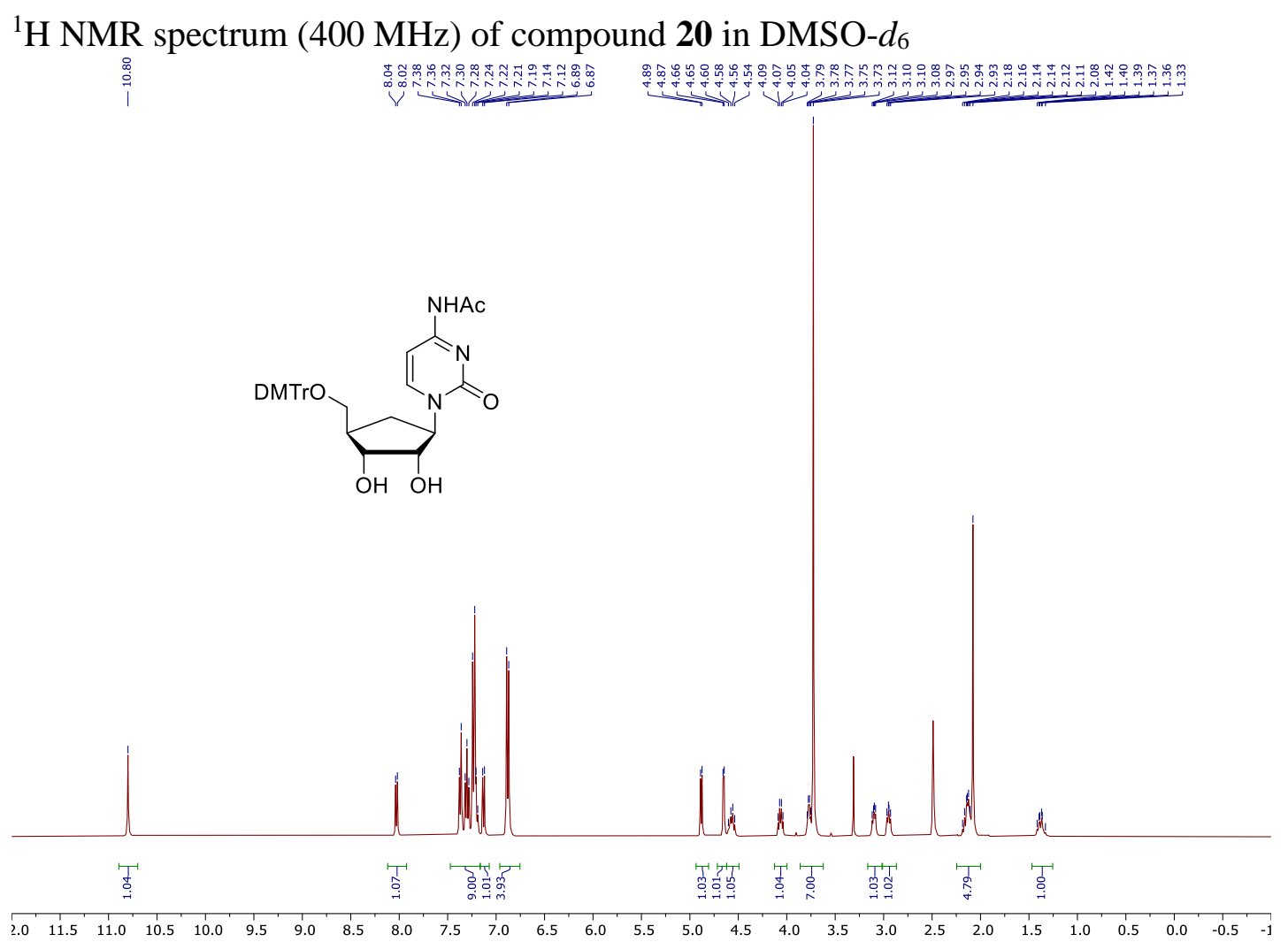

${ }^{13} \mathrm{C}$ NMR spectrum (126 MHz) of compound 20 in DMSO-d

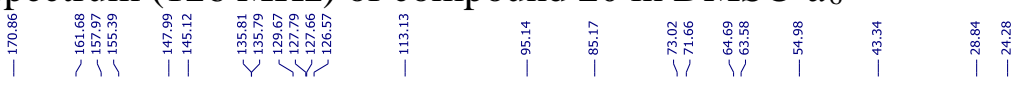

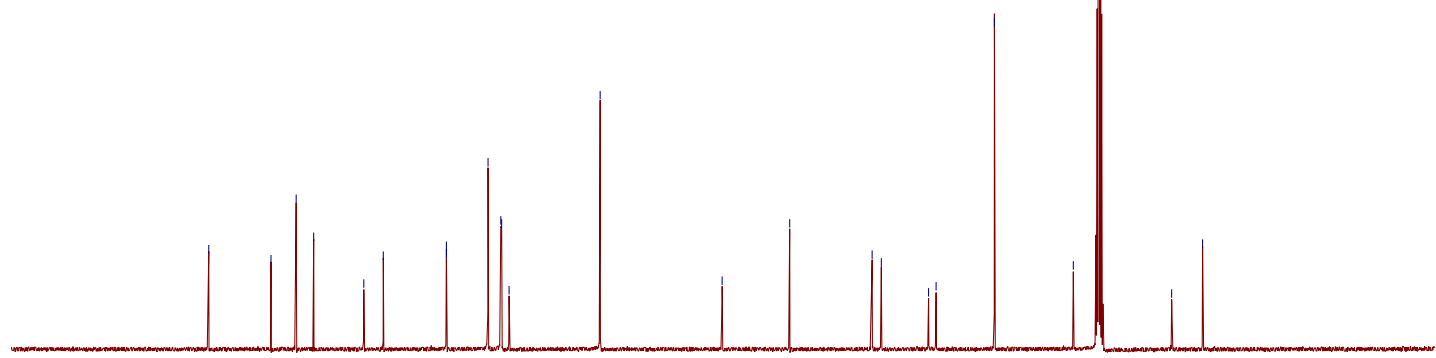

\begin{tabular}{llllllllllllllllllllll}
\hline & 190 & 180 & 170 & 160 & 150 & 140 & 130 & 120 & 110 & 100 & 90 & 80 & 70 & 60 & 50 & 40 & 30 & 20 & 10 & 0 & -1
\end{tabular} 


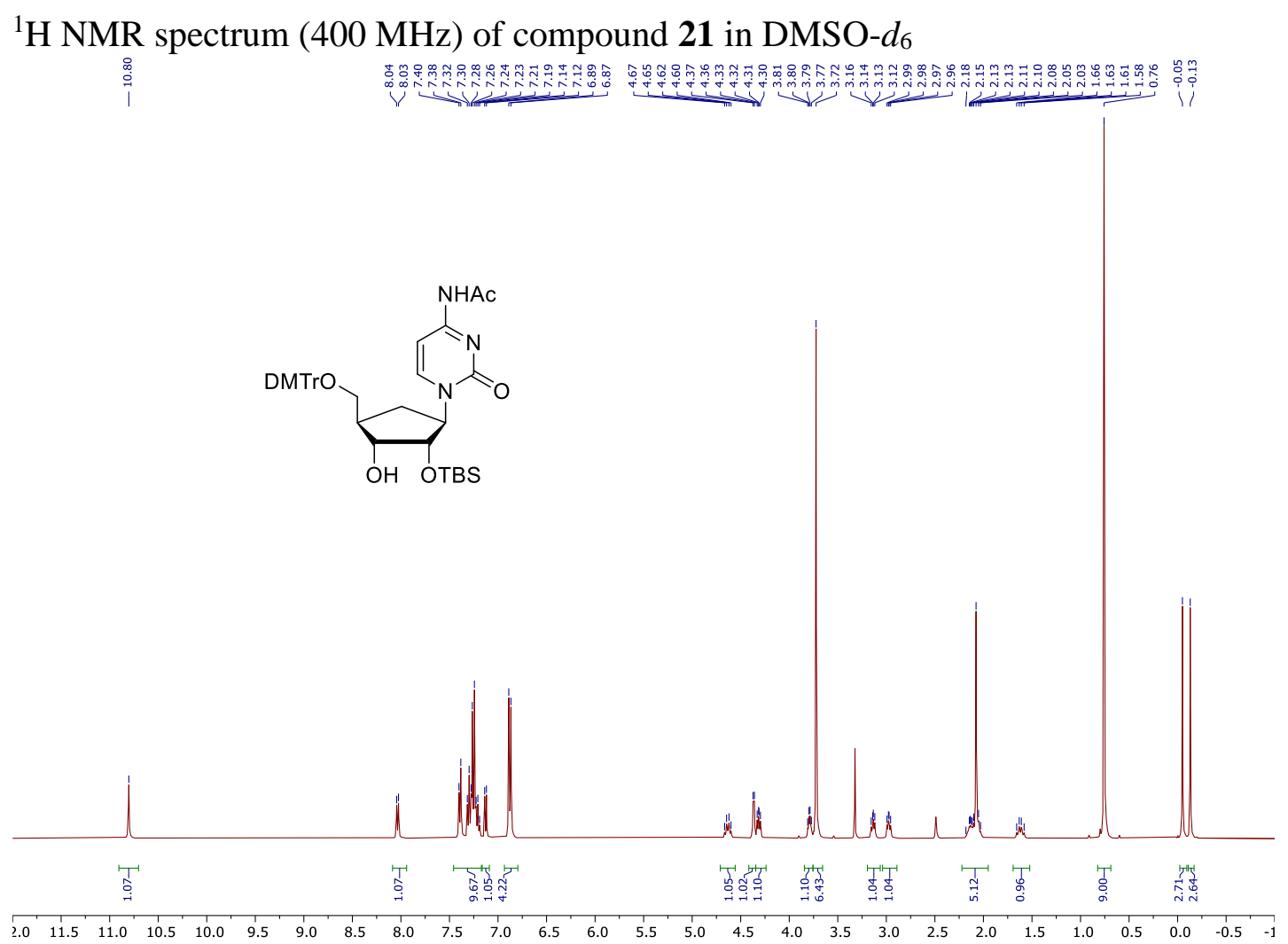

${ }^{13} \mathrm{C}$ NMR spectrum (101 MHz) of compound 21 in DMSO- $d_{6}$

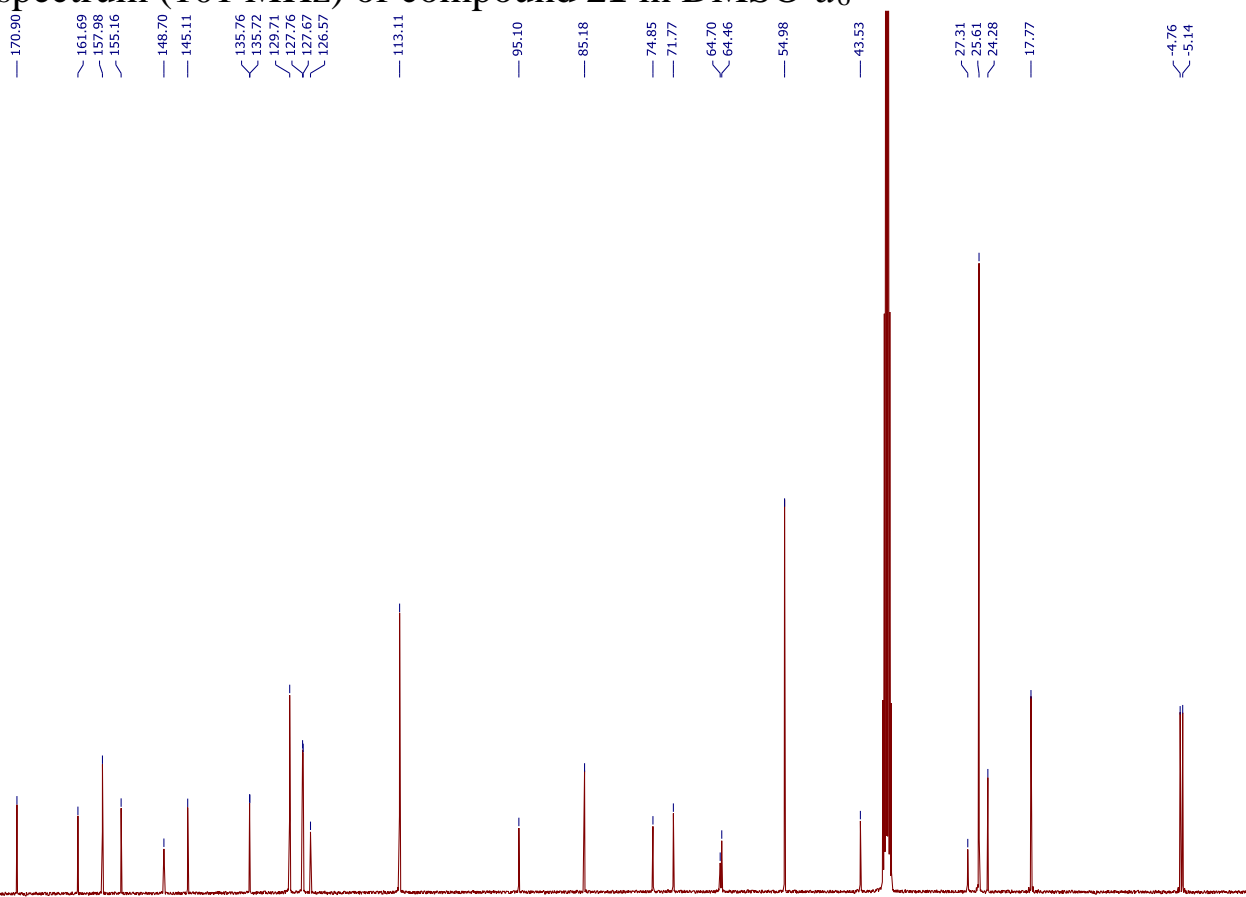

\begin{tabular}{llllllllllllllllllllllll}
\hline 00 & 190 & 180 & 170 & 160 & 150 & 140 & 130 & 120 & 110 & 100 & 90 & 80 & 70 & 60 & 50 & 40 & 30 & 20 & 10 & 0 & -10
\end{tabular} 
${ }^{1} \mathrm{H}-{ }^{1} \mathrm{H}$ COSY spectrum of compound 21 in DMSO- $d_{6}$
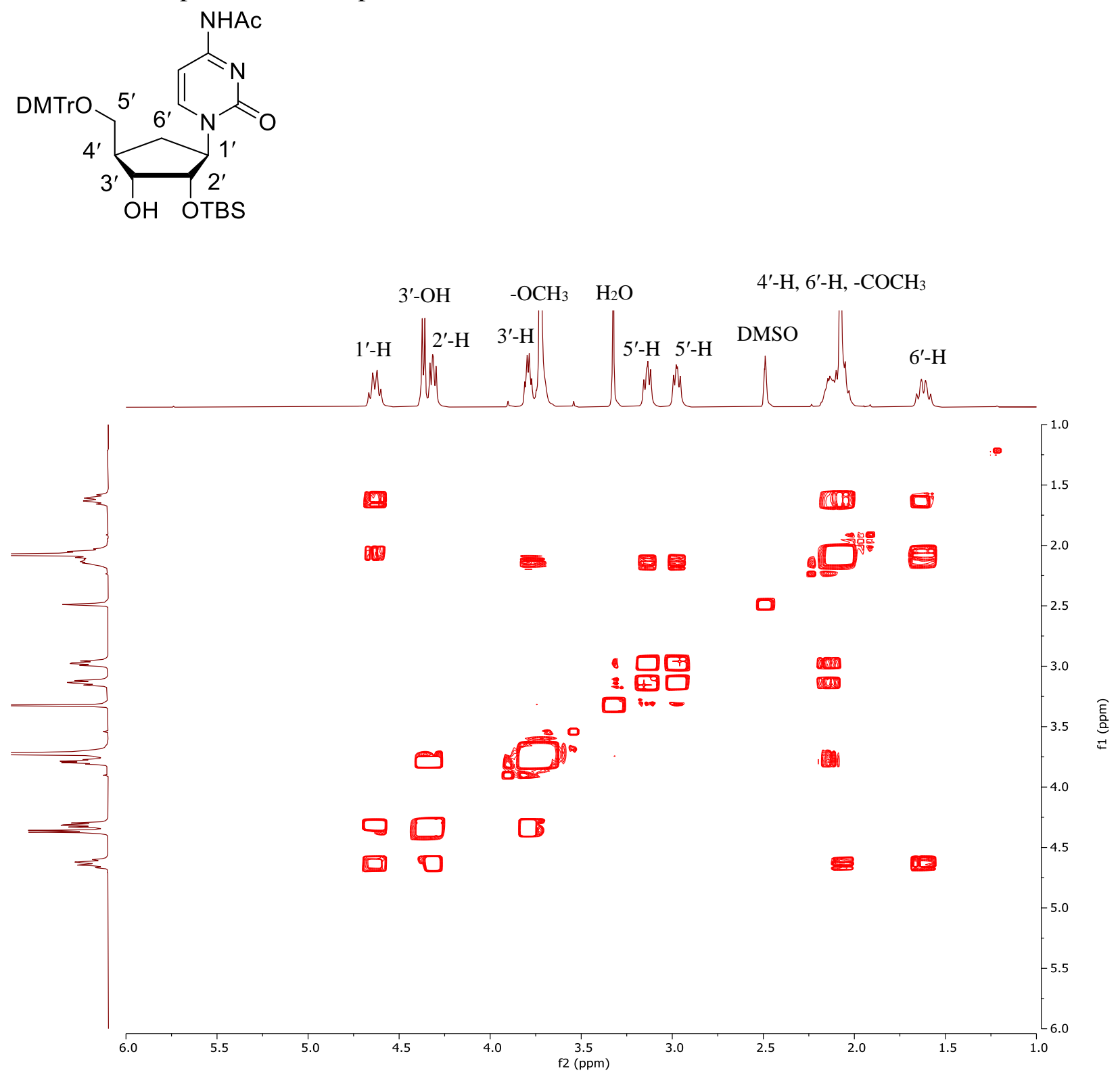
${ }^{1} \mathrm{H}$ NMR spectrum (400 MHz) of compound 22 in DMSO- $d_{6}$

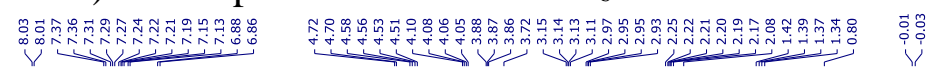
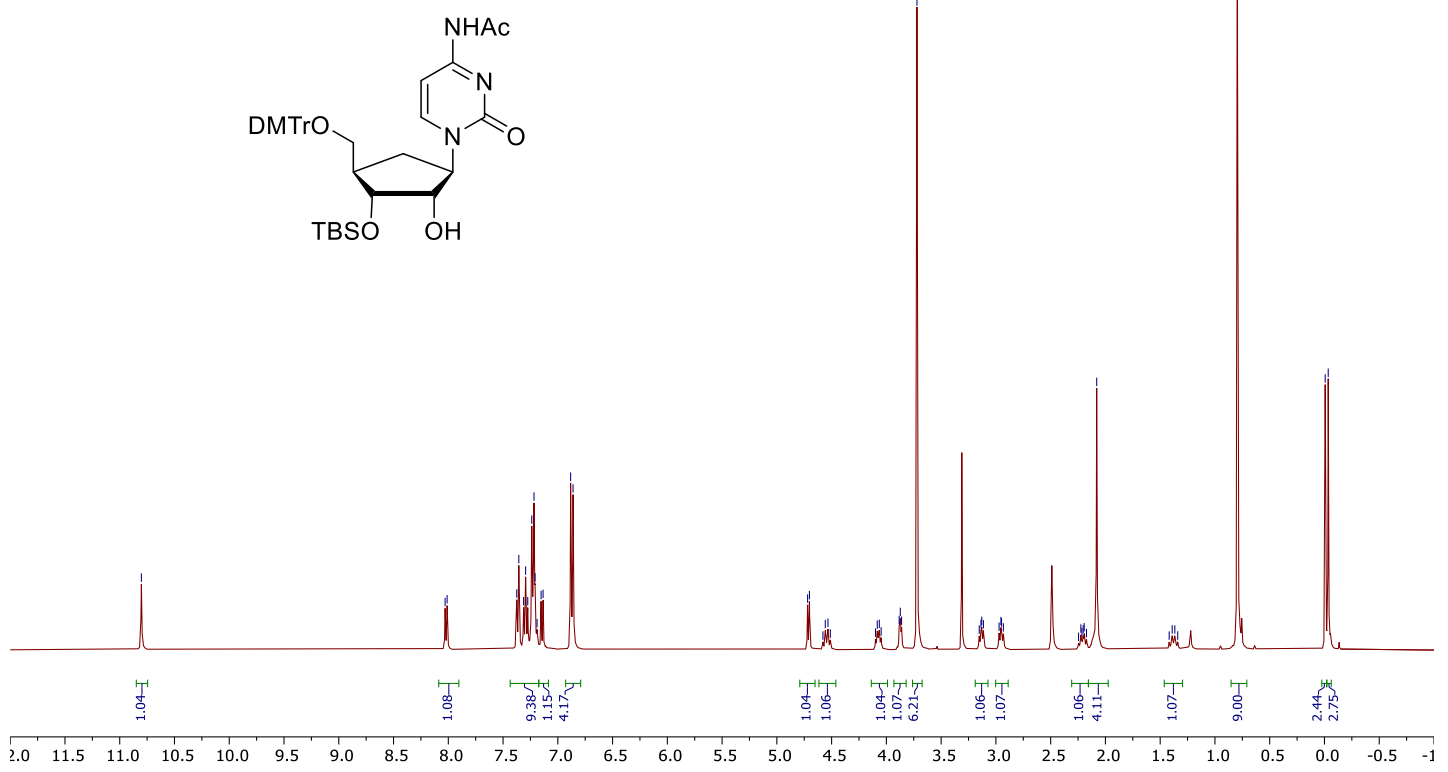

${ }^{13} \mathrm{C}$ NMR spectrum (101 MHz) of compound 22 in DMSO- $d_{6}$

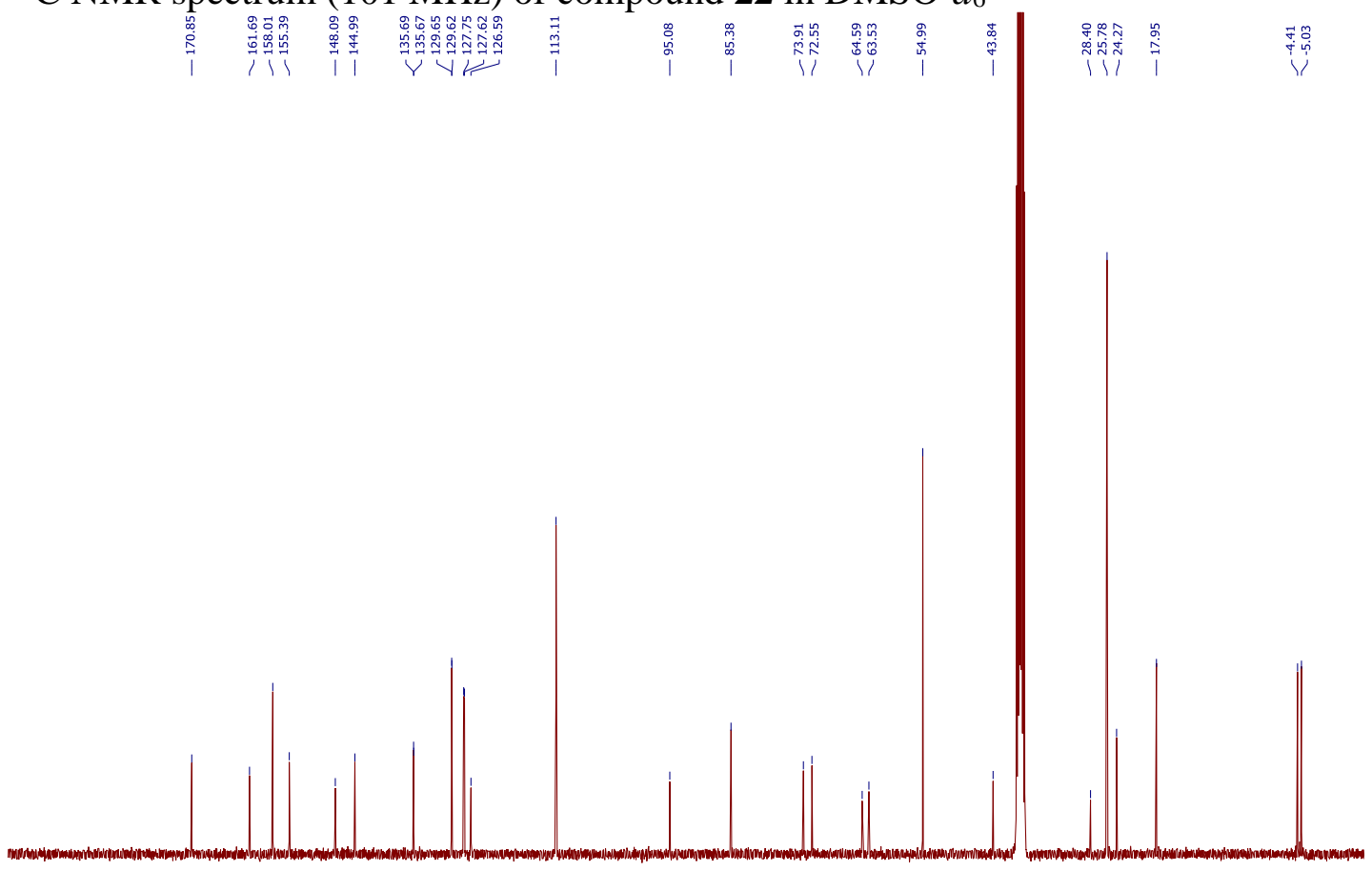

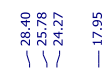

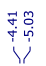

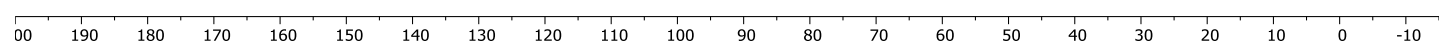

- S45 - 
${ }^{1} \mathrm{H}-{ }^{1} \mathrm{H}$ COSY spectrum of compound 22 in DMSO- $d_{6}$
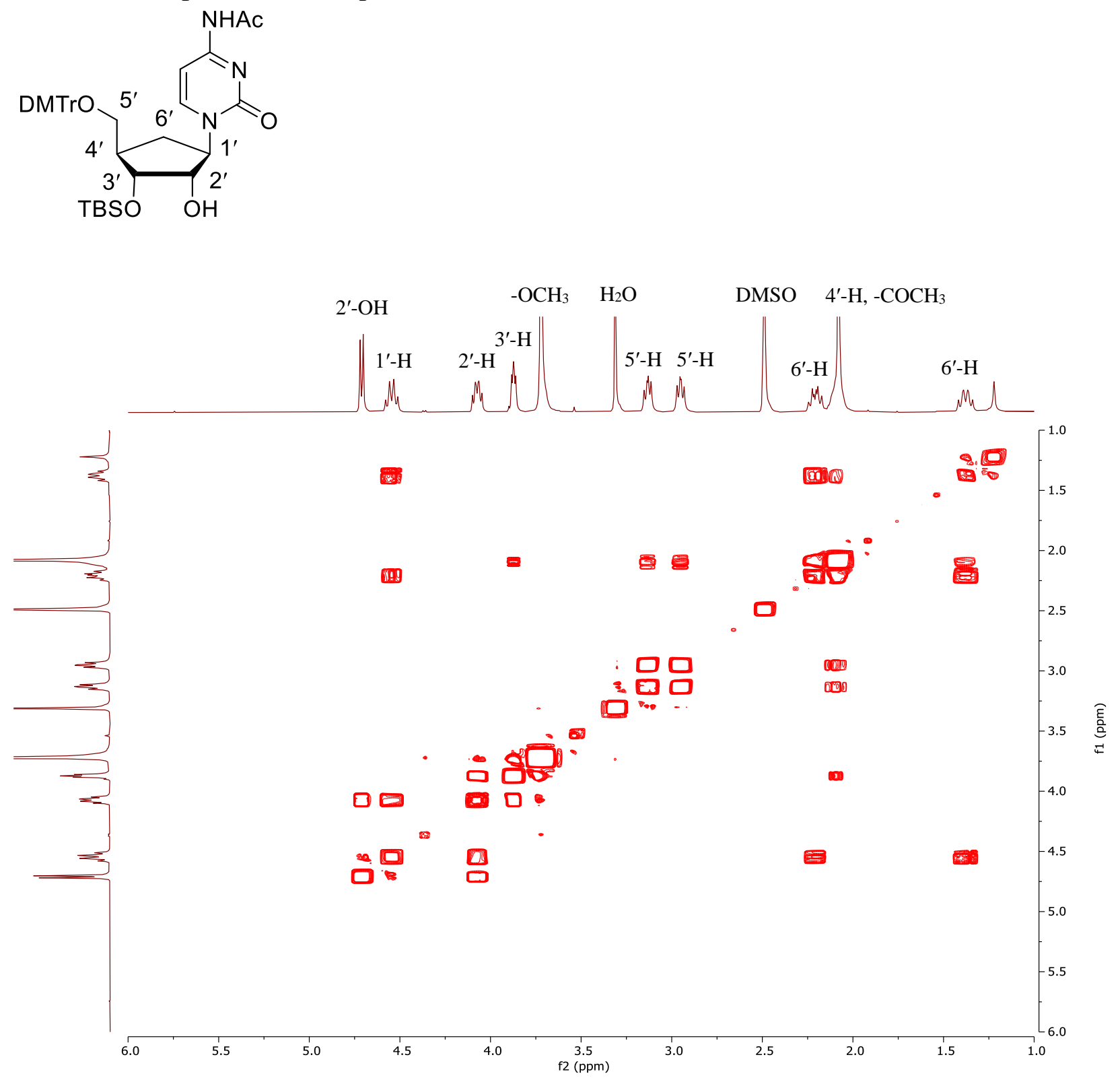
${ }^{1} \mathrm{H}$ NMR spectrum (400 MHz) of compound 3 in $\mathrm{CD}_{3} \mathrm{CN}$

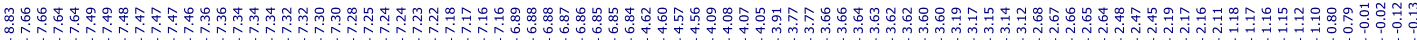
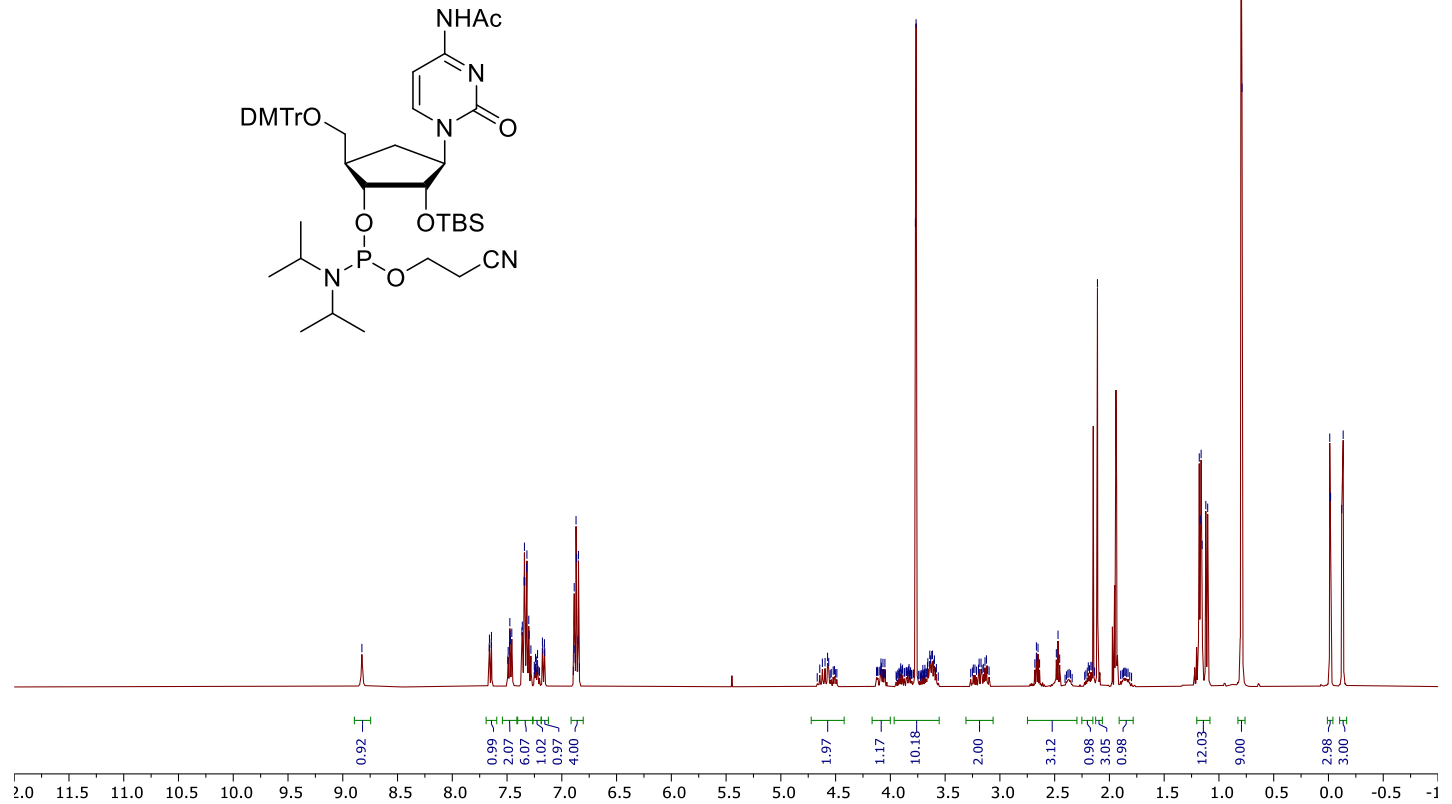

${ }^{13} \mathrm{C}$ NMR spectrum (101 MHz) of compound 3 in $\mathrm{CD}_{3} \mathrm{CN}$

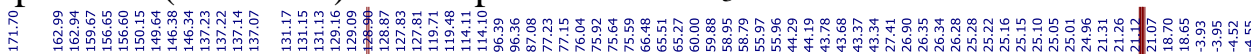

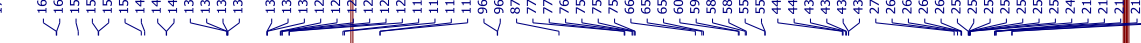

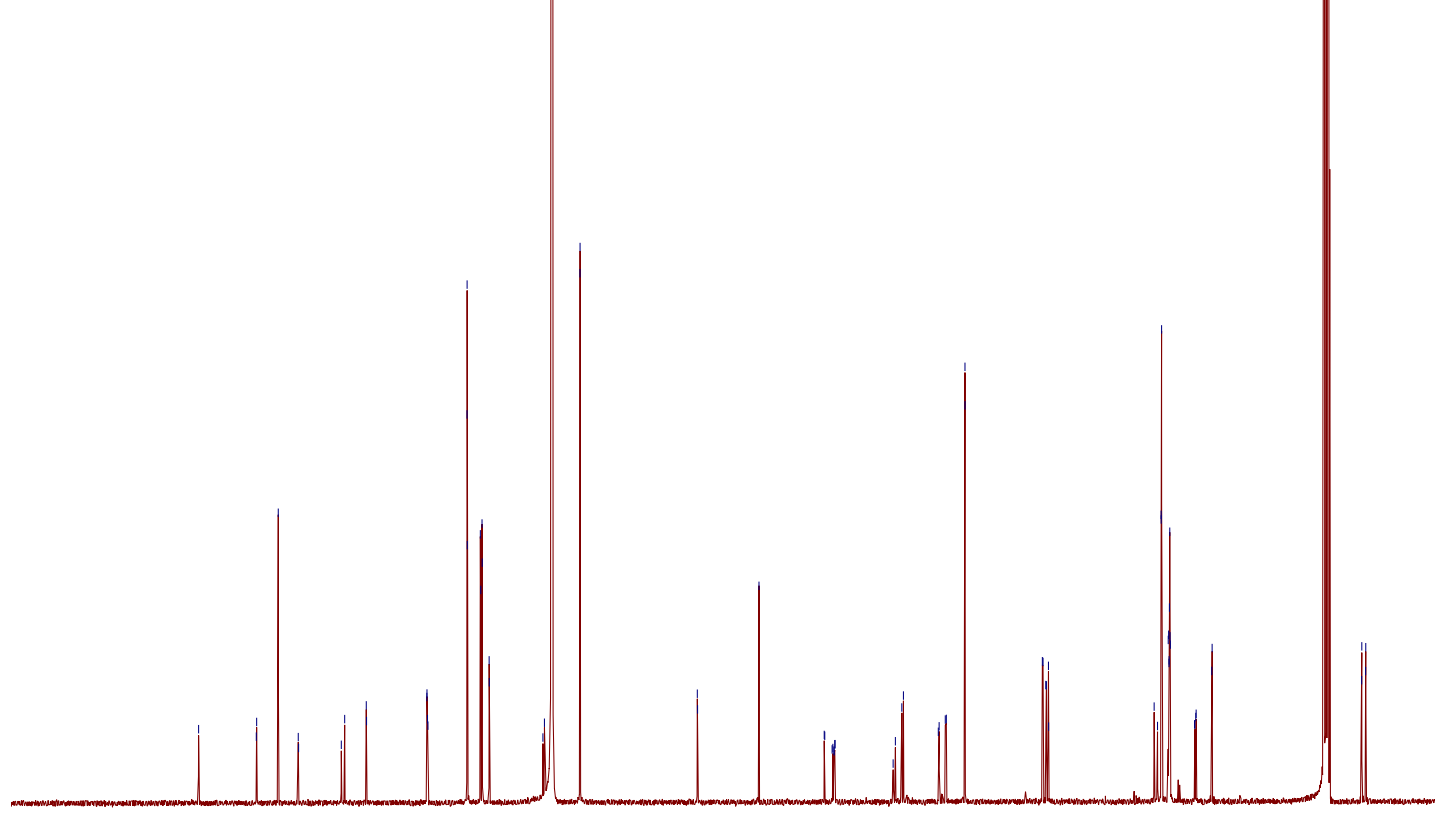

\begin{tabular}{llllllllllllllllllllllll}
\hline 00 & 190 & 180 & 170 & 160 & 150 & 140 & 130 & 120 & 110 & 100 & 90 & 80 & 70 & 60 & 50 & 40 & 30 & 20 & 10 & 0 & -10
\end{tabular} 


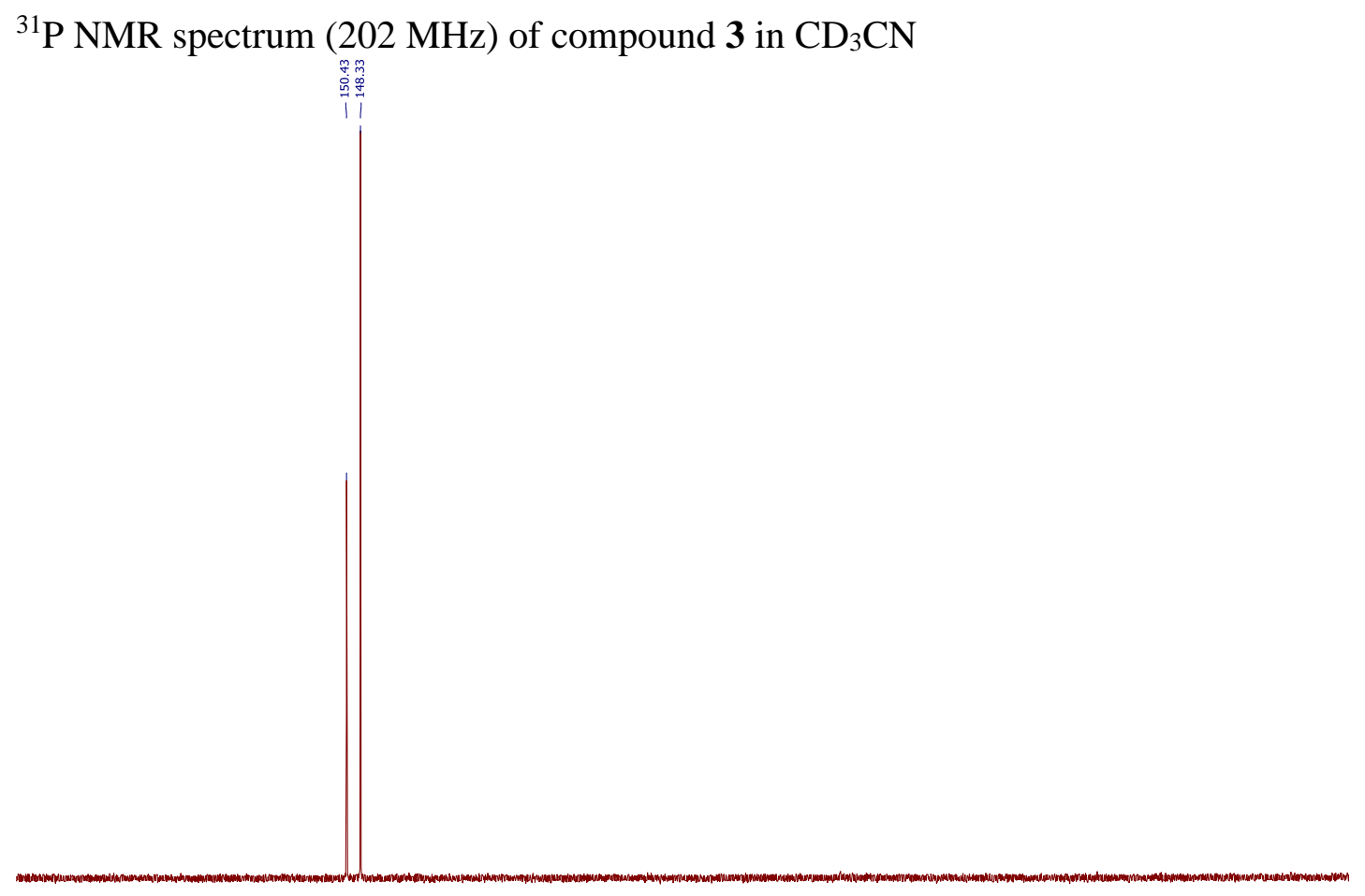


${ }^{1} \mathrm{H}$ NMR spectrum (400 MHz) of compound 4 in $\mathrm{CD}_{3} \mathrm{CN}$

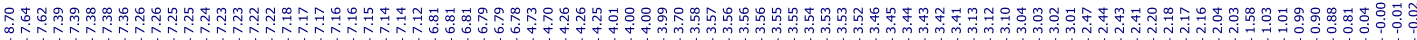

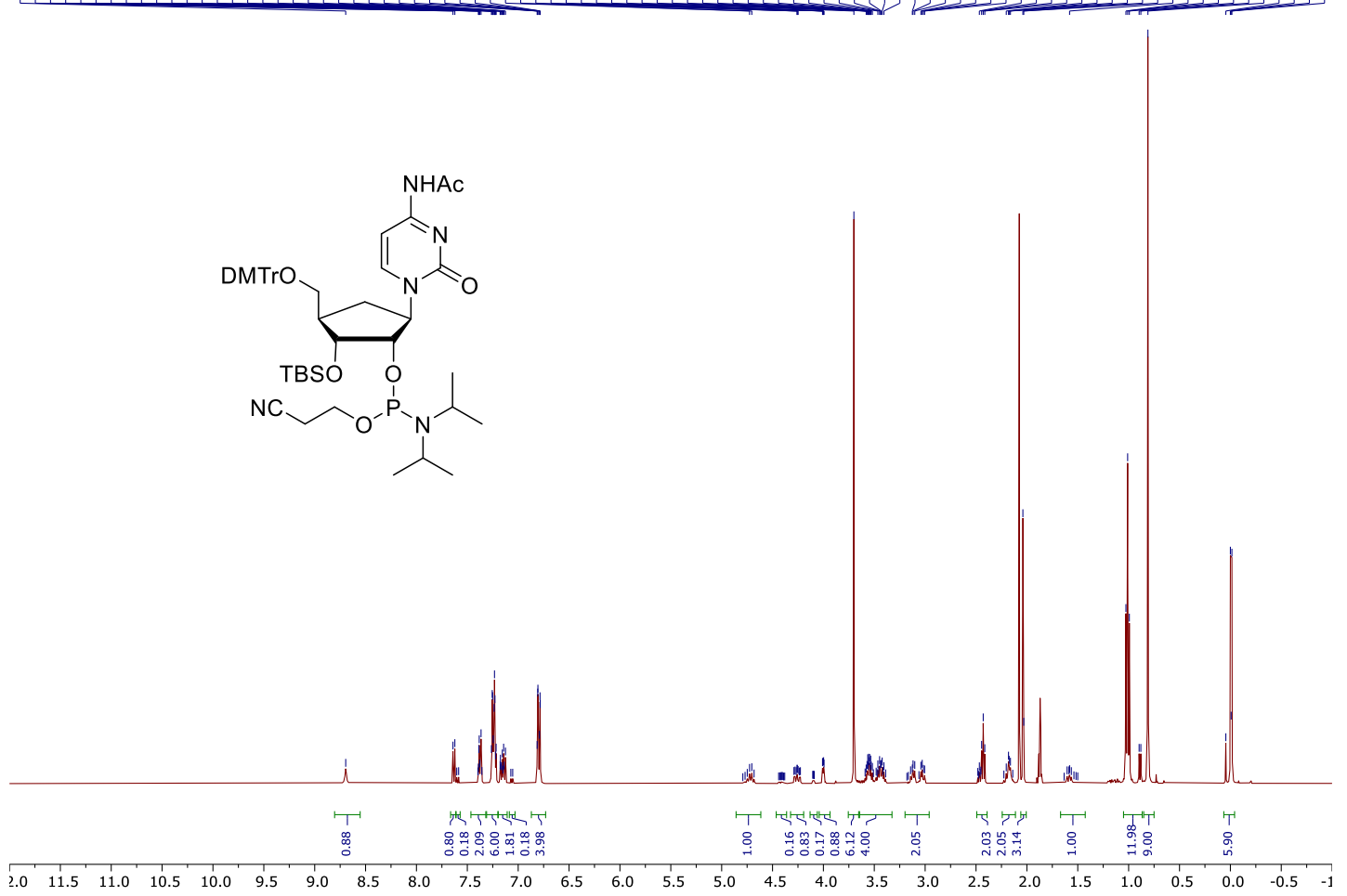

${ }^{13} \mathrm{C}$ NMR spectrum (101 MHz) of compound 4 in $\mathrm{CD}_{3} \mathrm{CN}$
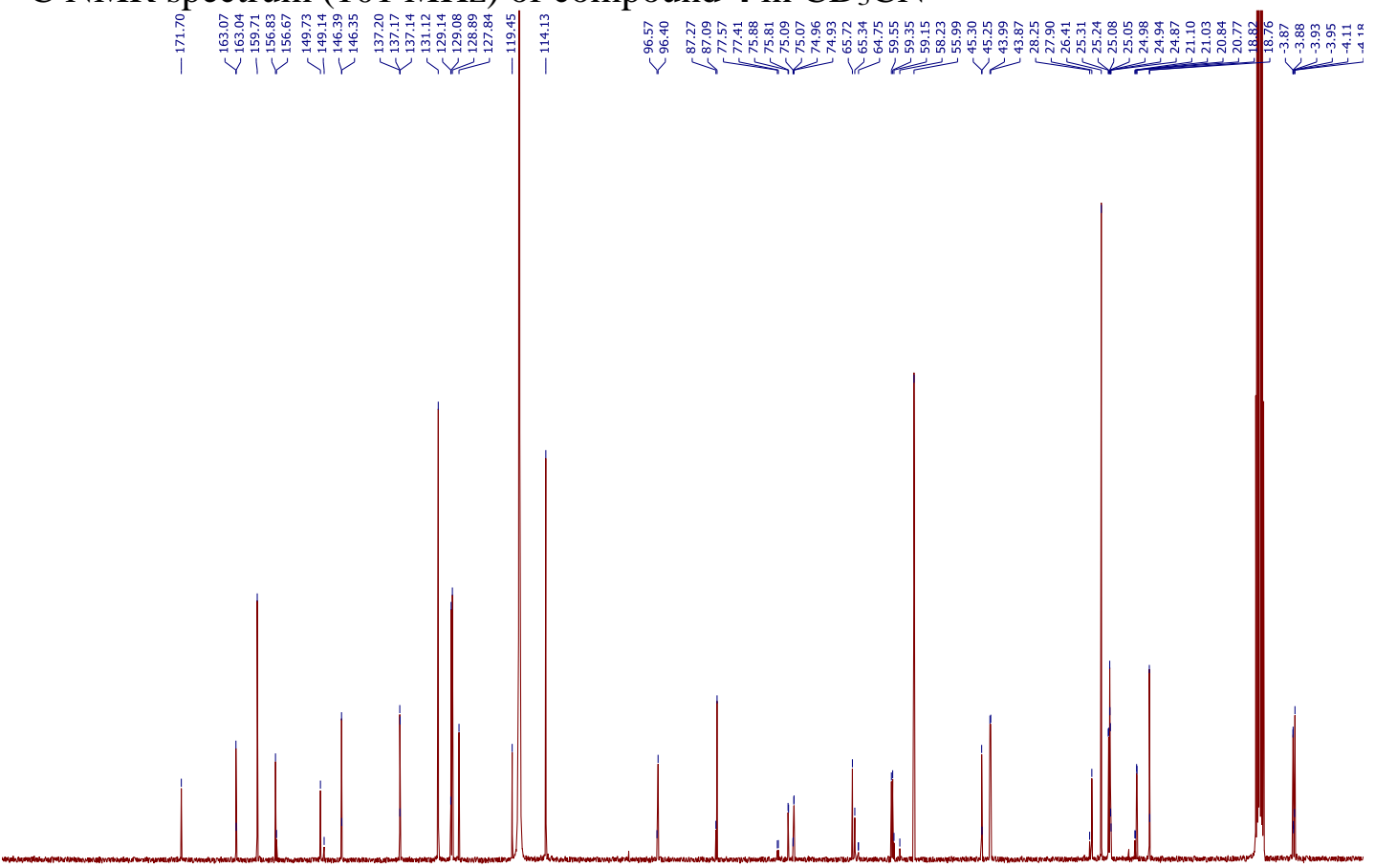

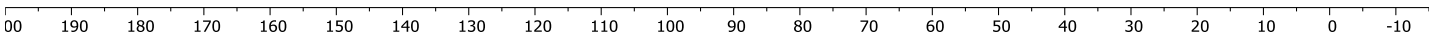



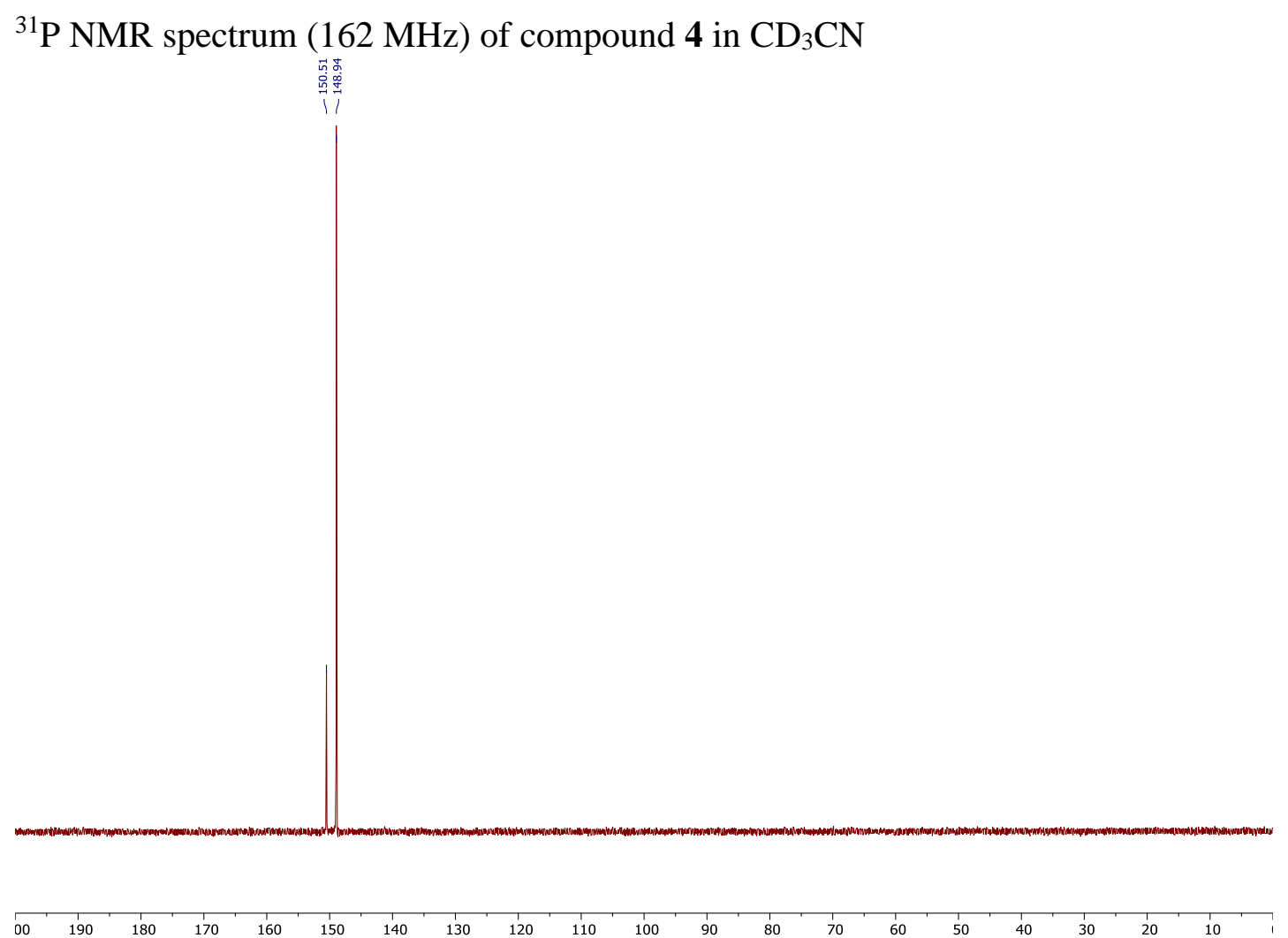
${ }^{1} \mathrm{H}$ NMR spectrum $(500 \mathrm{MHz})$ of compound 23 in DMSO- $d_{6}$

罚品

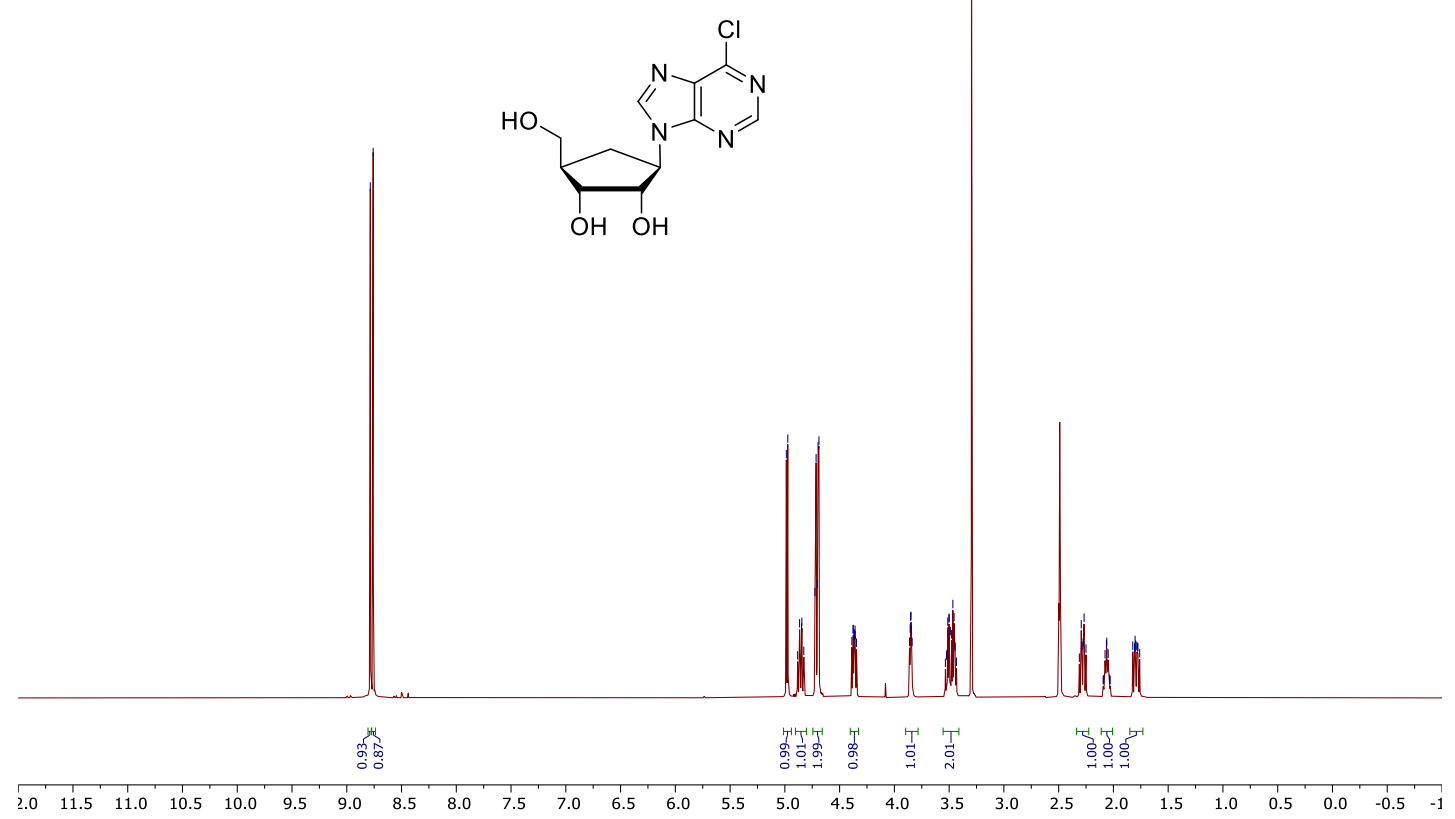

${ }^{13} \mathrm{C}$ NMR spectrum $(126 \mathrm{MHz})$ of compound 23 in DMSO- $d_{6}$

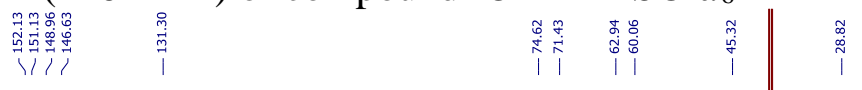

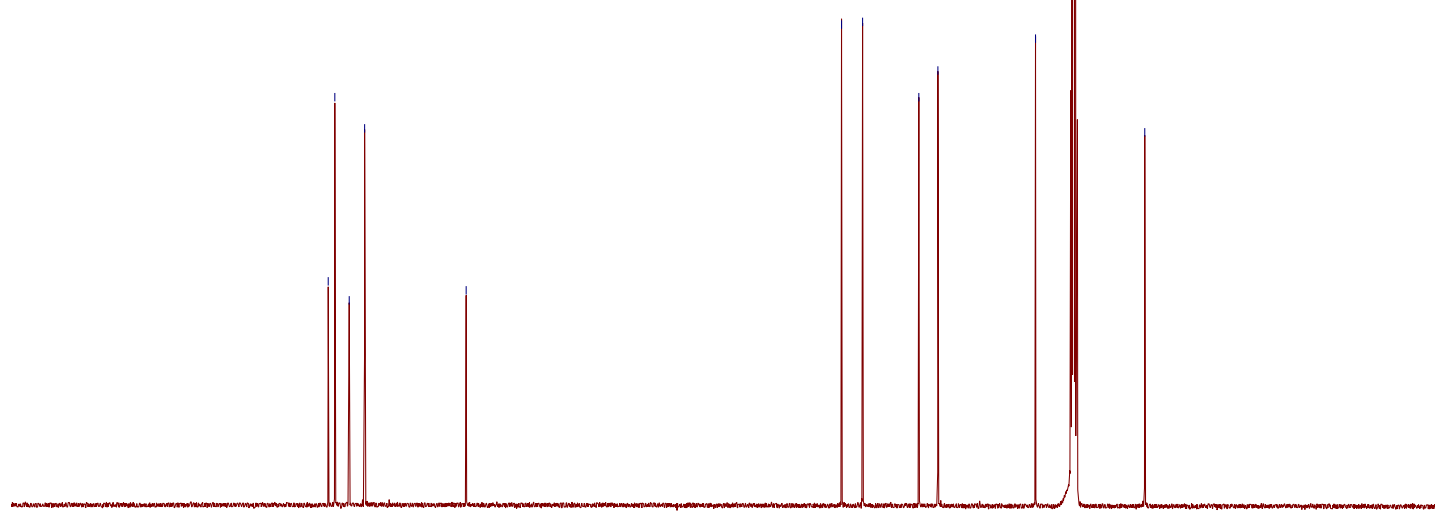

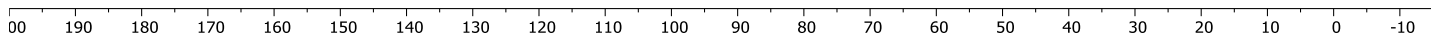


${ }^{1} \mathrm{H}$ NMR spectrum $(400 \mathrm{MHz})$ of compound 24 in DMSO- $d_{6}$
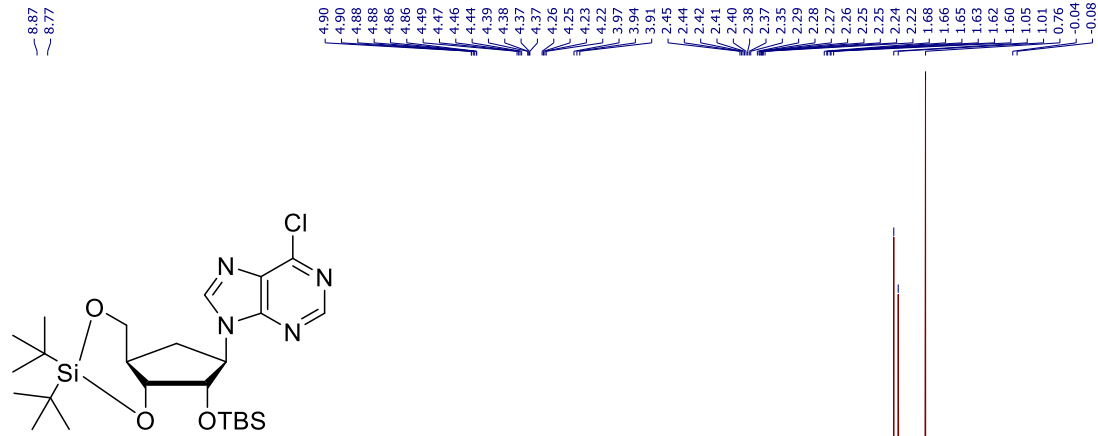

Hith

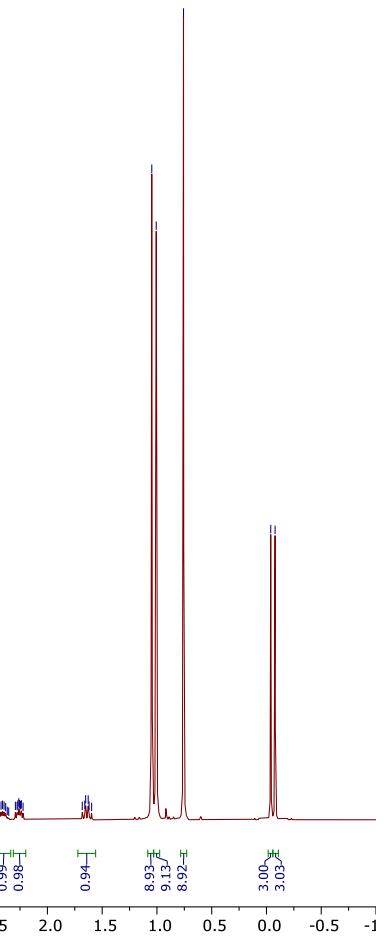

${ }^{13} \mathrm{C}$ NMR spectrum $(126 \mathrm{MHz})$ of compound 24 in DMSO- $d_{6}$

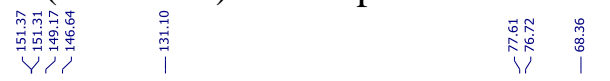

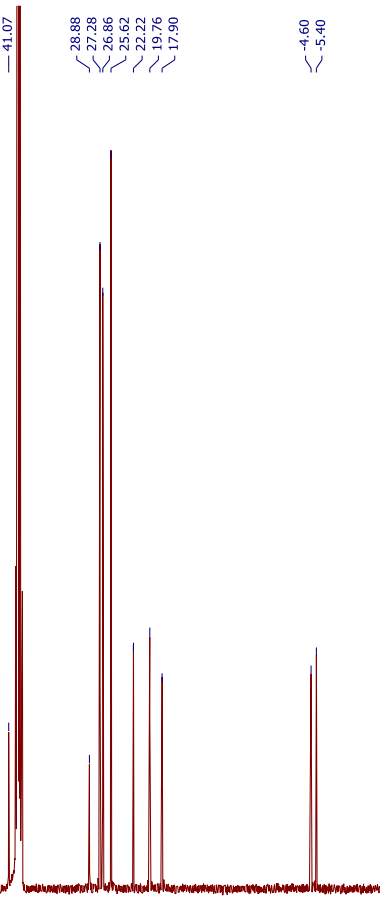

00
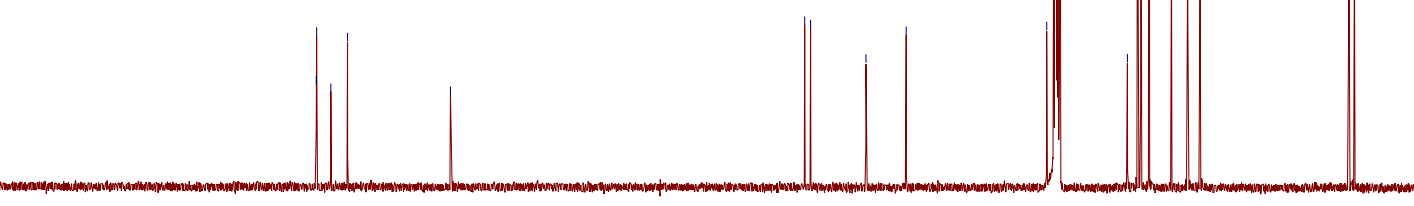

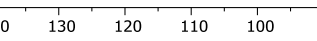

\begin{tabular}{lllllll}
1 & 1 & 1 & 1 & 1 & 1 & 10 \\
\hline 0 & 40 & 30 & 20 & 10 & 0 & -10
\end{tabular} 
${ }^{1} \mathrm{H}$ NMR spectrum (500 MHz) of compound 25 in DMSO- $d_{6}$

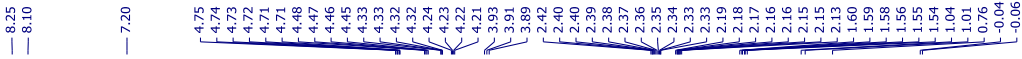
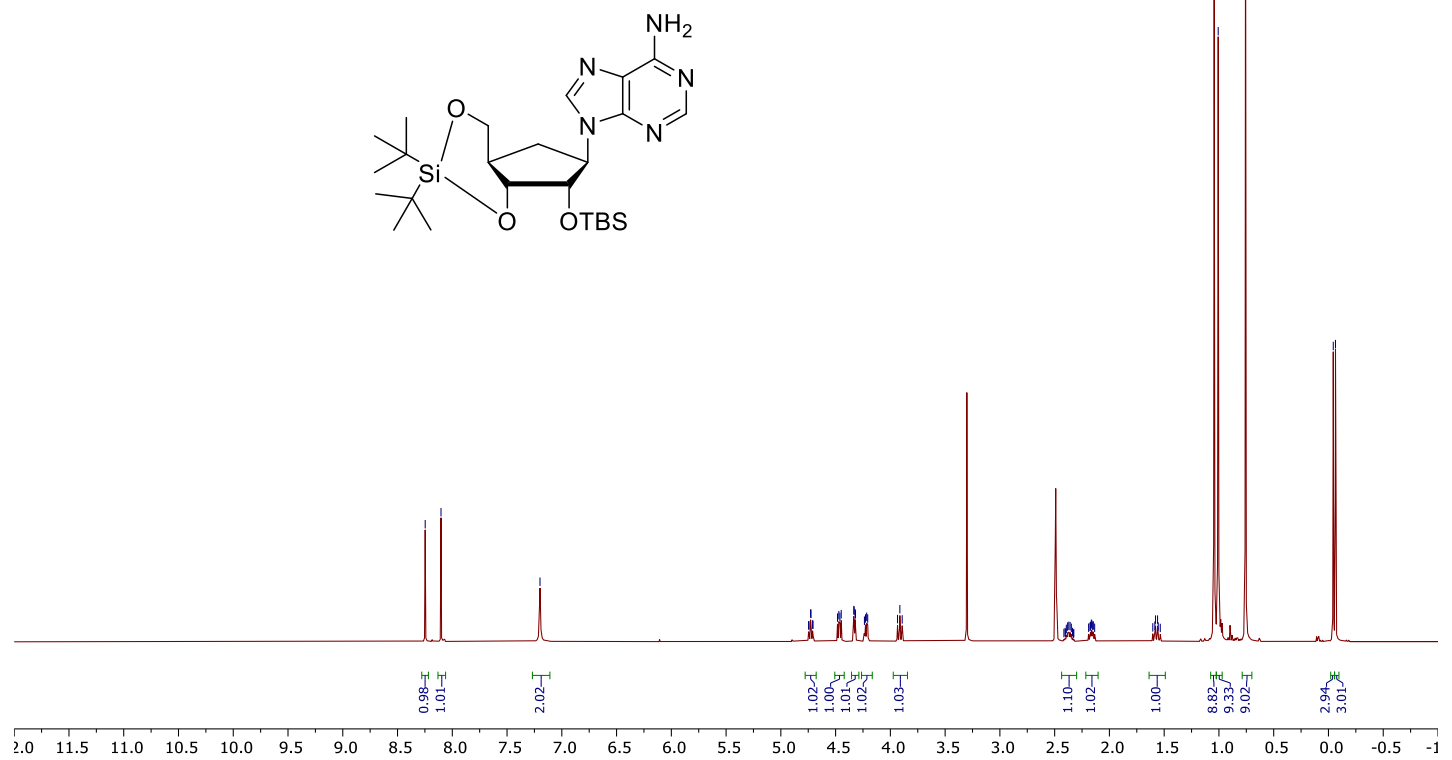

${ }^{13} \mathrm{C}$ NMR spectrum (126 MHz) of compound 25 in DMSO- $d_{6}$

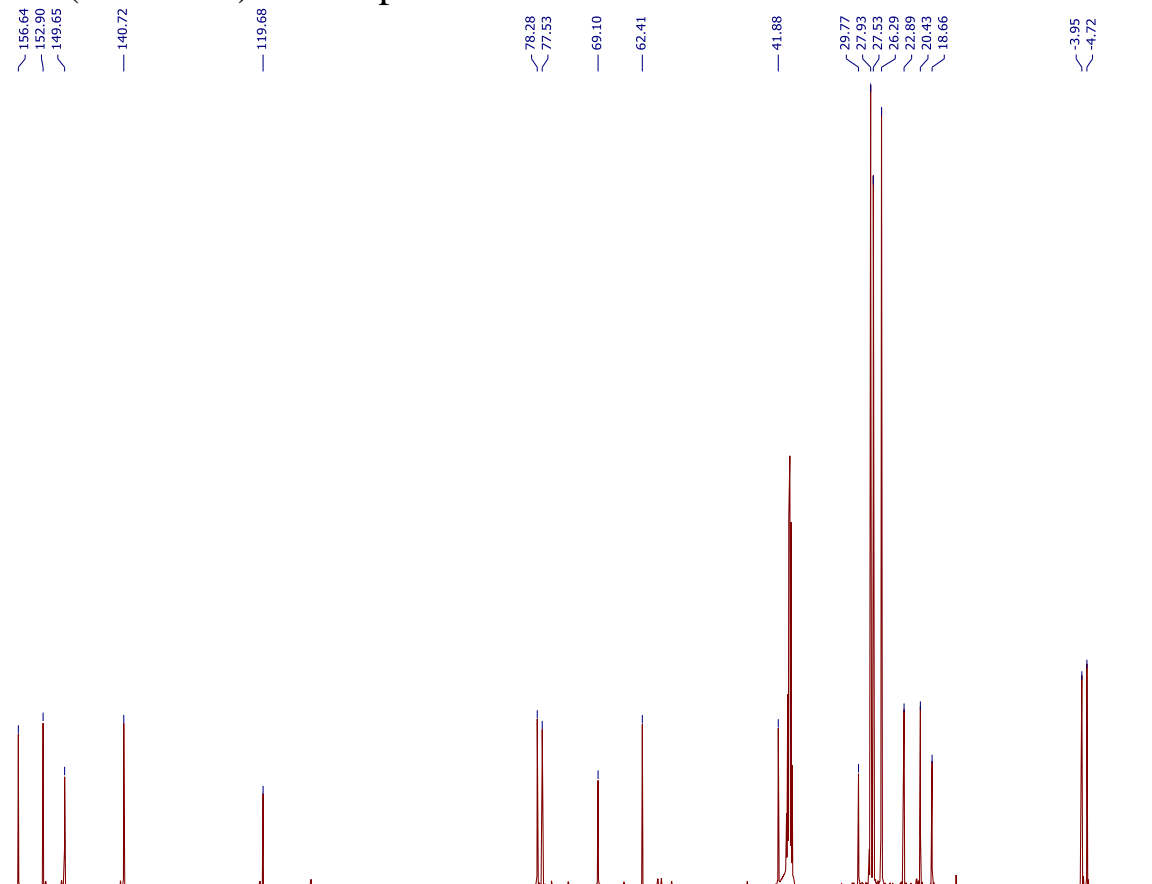

\begin{tabular}{lllllllllllllllllllllll}
\hline 00 & 190 & 180 & 170 & 160 & 150 & 140 & 130 & 120 & 110 & 100 & 90 & 80 & 70 & 60 & 50 & 40 & 30 & 20 & 10 & 0 & -10
\end{tabular} 
${ }^{1} \mathrm{H}$ NMR spectrum $(500 \mathrm{MHz})$ of compound 26 in DMSO- $d_{6}$

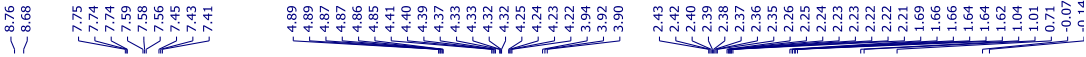
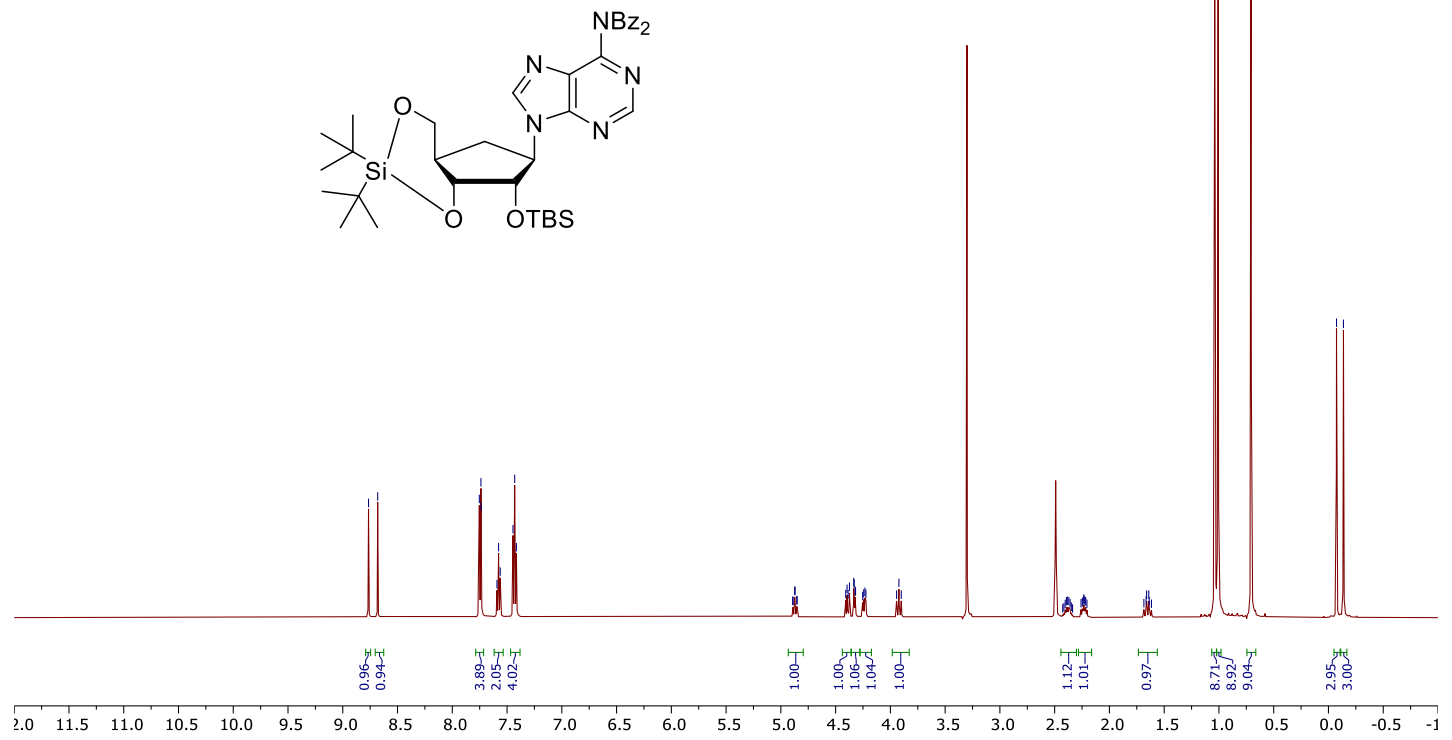

${ }^{13} \mathrm{C}$ NMR spectrum (101 MHz) of compound 26 in DMSO- $d_{6}$

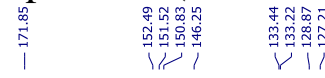
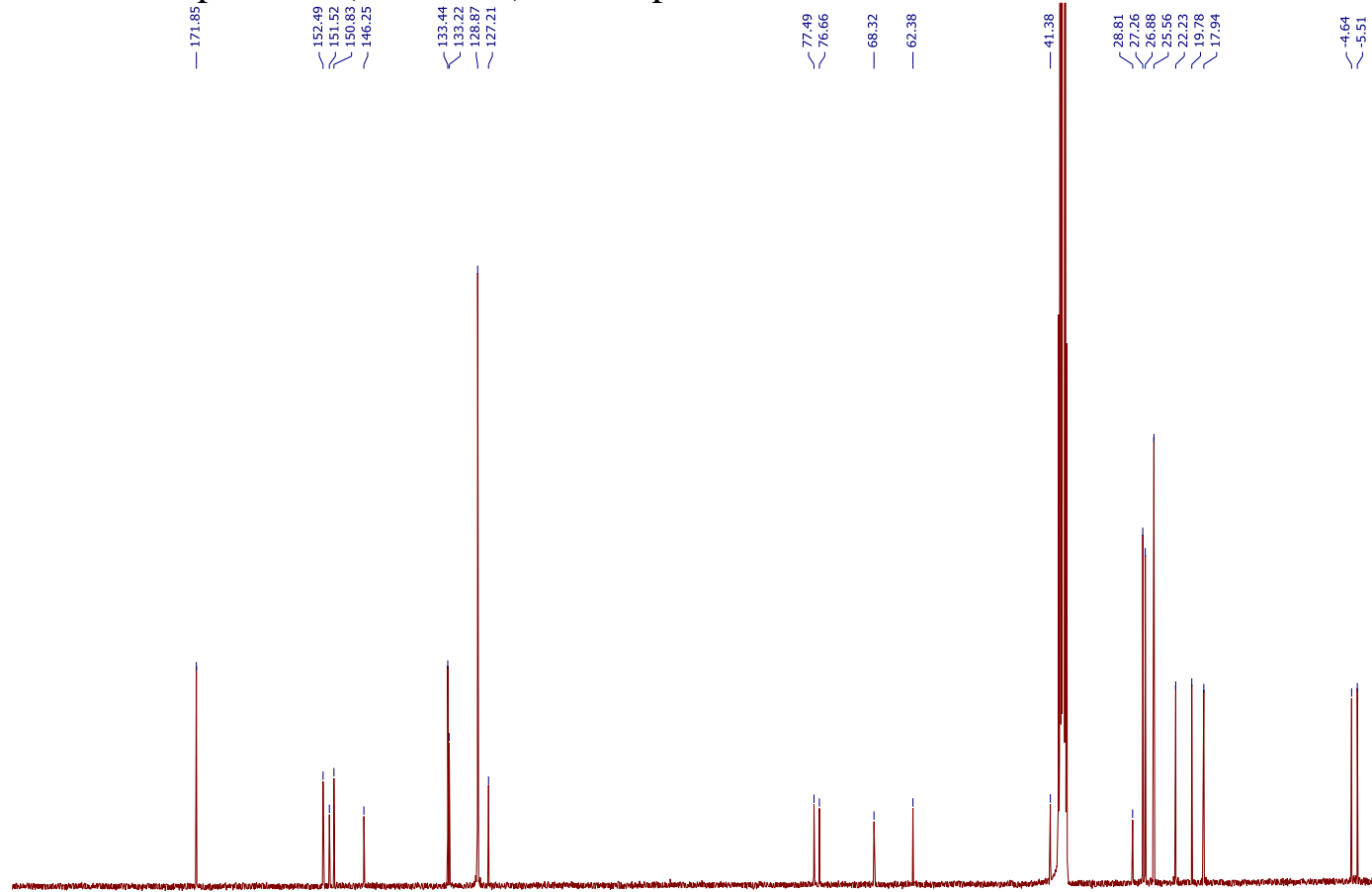
${ }^{1} \mathrm{H}$ NMR spectrum $(500 \mathrm{MHz})$ of compound 27 in DMSO- $d_{6}$ $\stackrel{\overbrace{}}{i}$
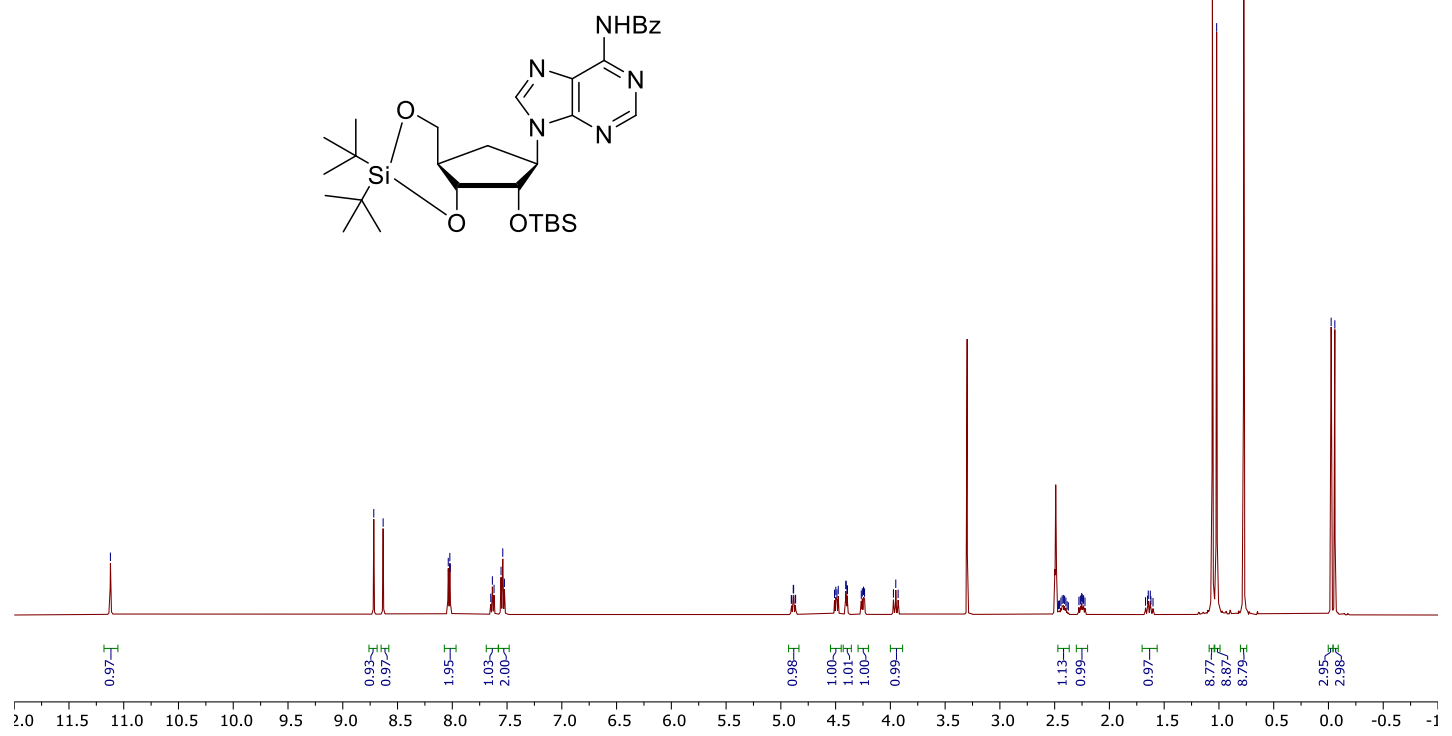

${ }^{13} \mathrm{C}$ NMR spectrum $(101 \mathrm{MHz})$ of compound 27 in DMSO- $d_{6}$

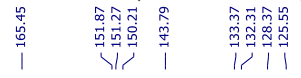


${ }^{1} \mathrm{H}$ NMR spectrum $(400 \mathrm{MHz})$ of compound 28 in DMSO- $d_{6}$

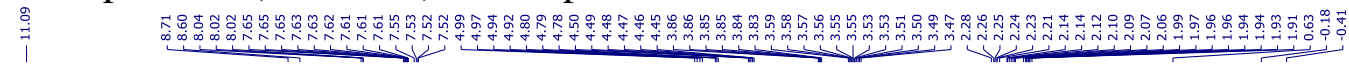
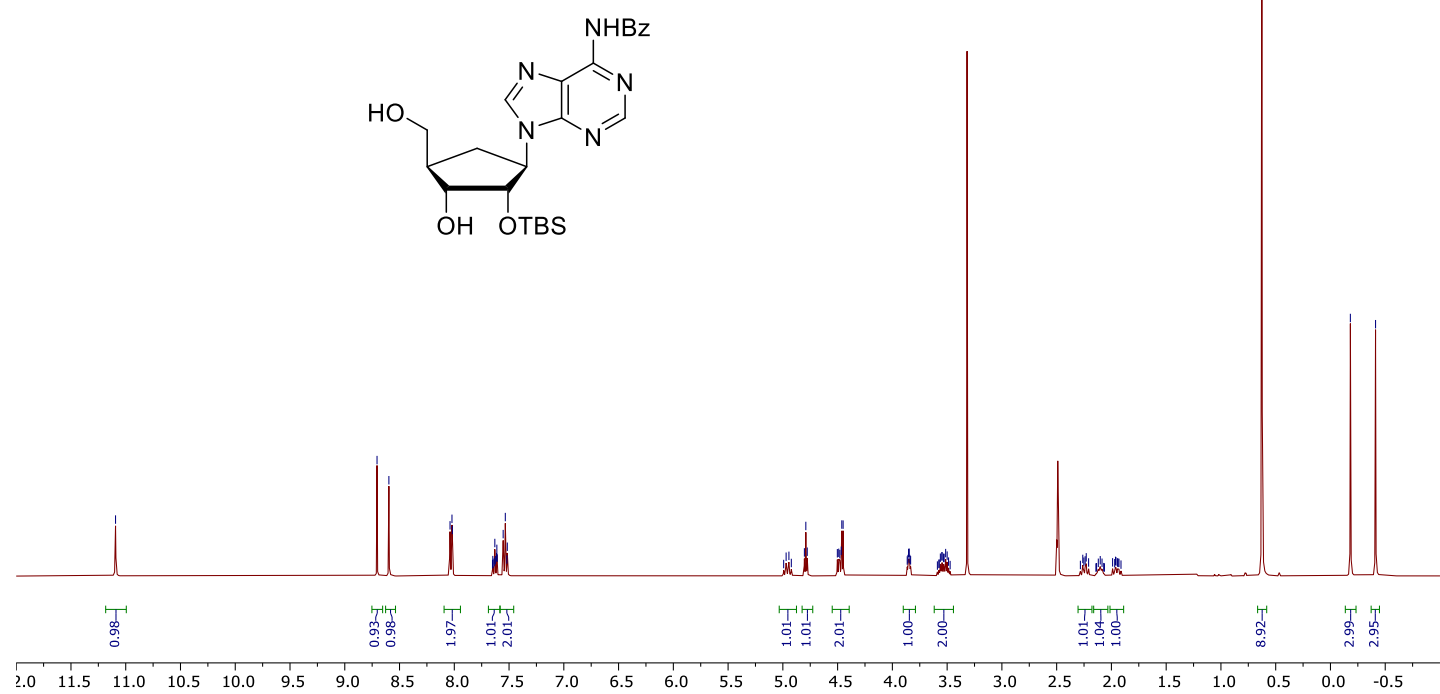

${ }^{13} \mathrm{C}$ NMR spectrum (126 MHz) of compound 28 in DMSO- $d_{6}$

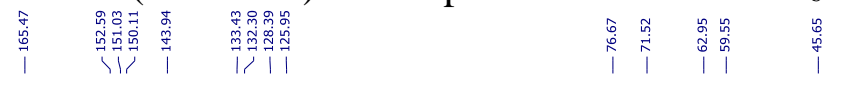
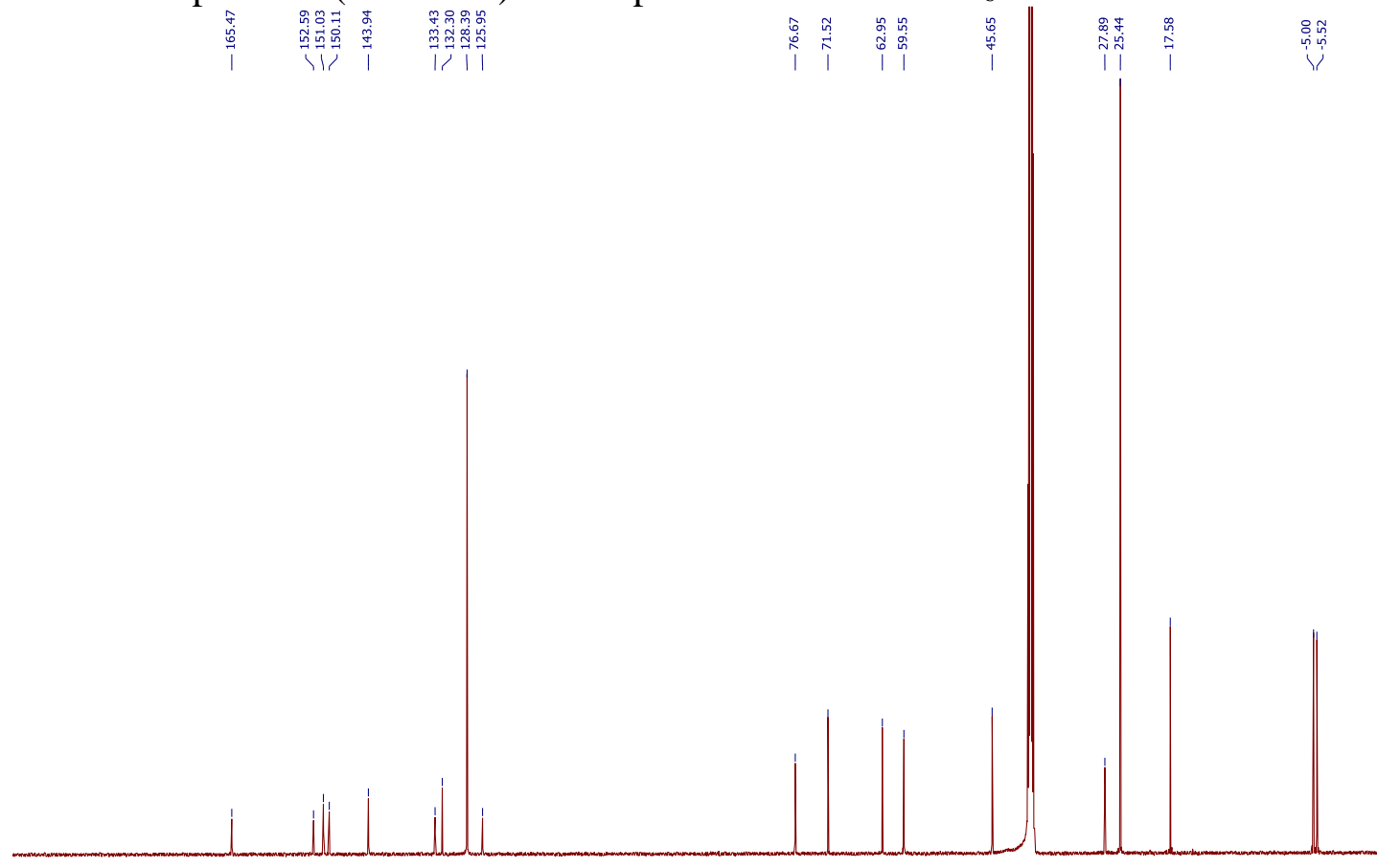

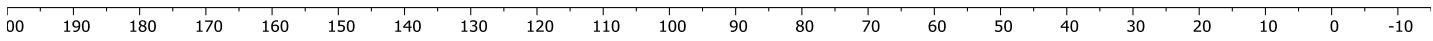


${ }^{1} \mathrm{H}-{ }^{1} \mathrm{H}$ COSY spectrum of compound 28 in DMSO- $d_{6}$

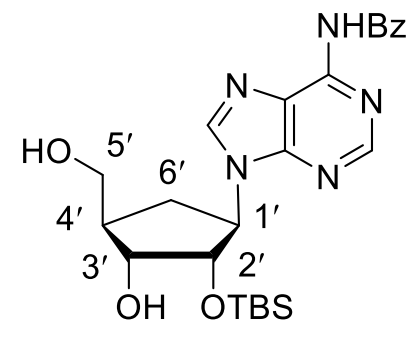

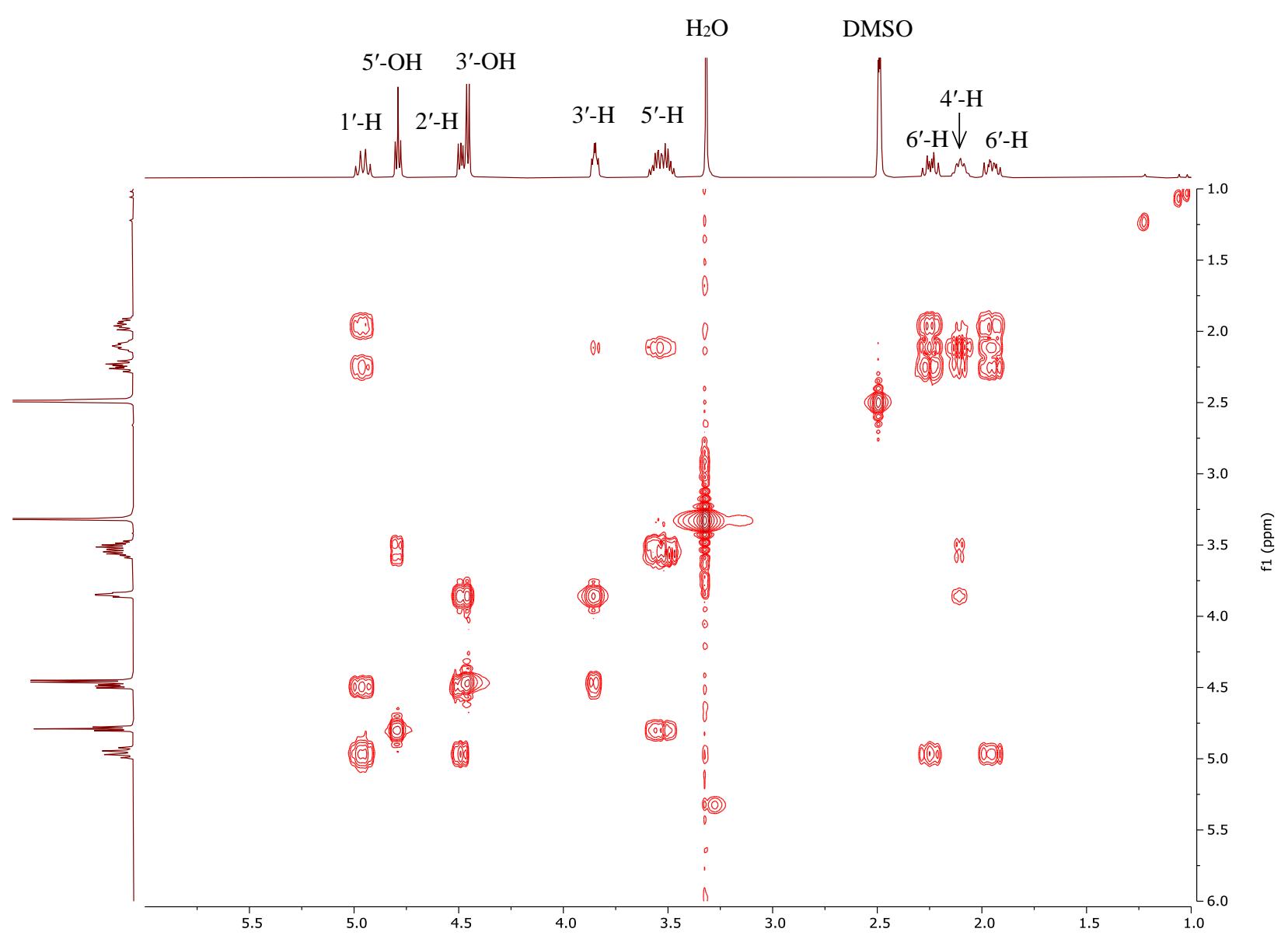


${ }^{1} \mathrm{H}$ NMR spectrum $(500 \mathrm{MHz})$ of compound 29 in DMSO- $d_{6}$
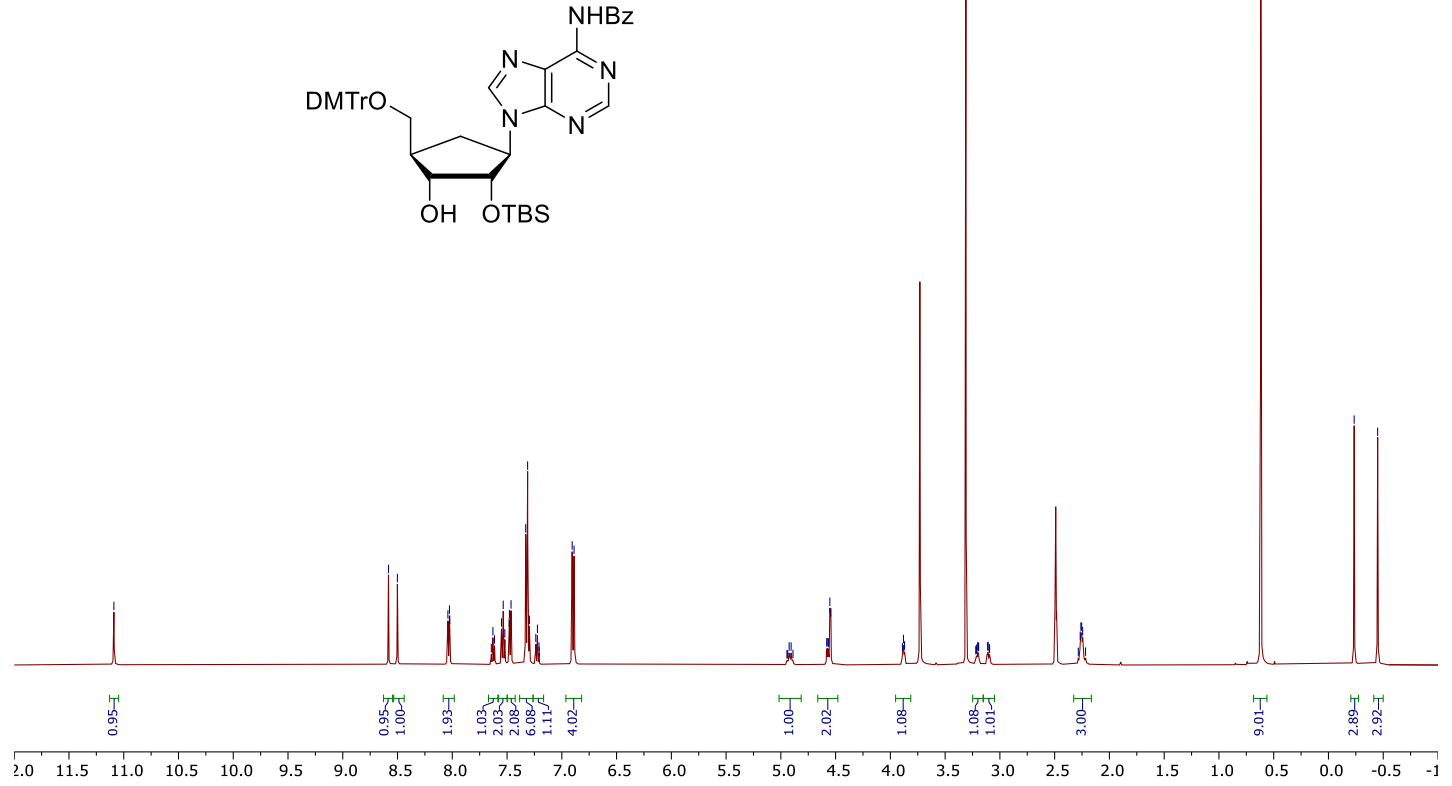

${ }^{13} \mathrm{C}$ NMR spectrum (101 MHz) of compound 29 in DMSO- $d_{6}$

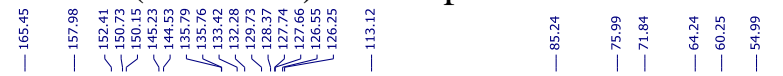

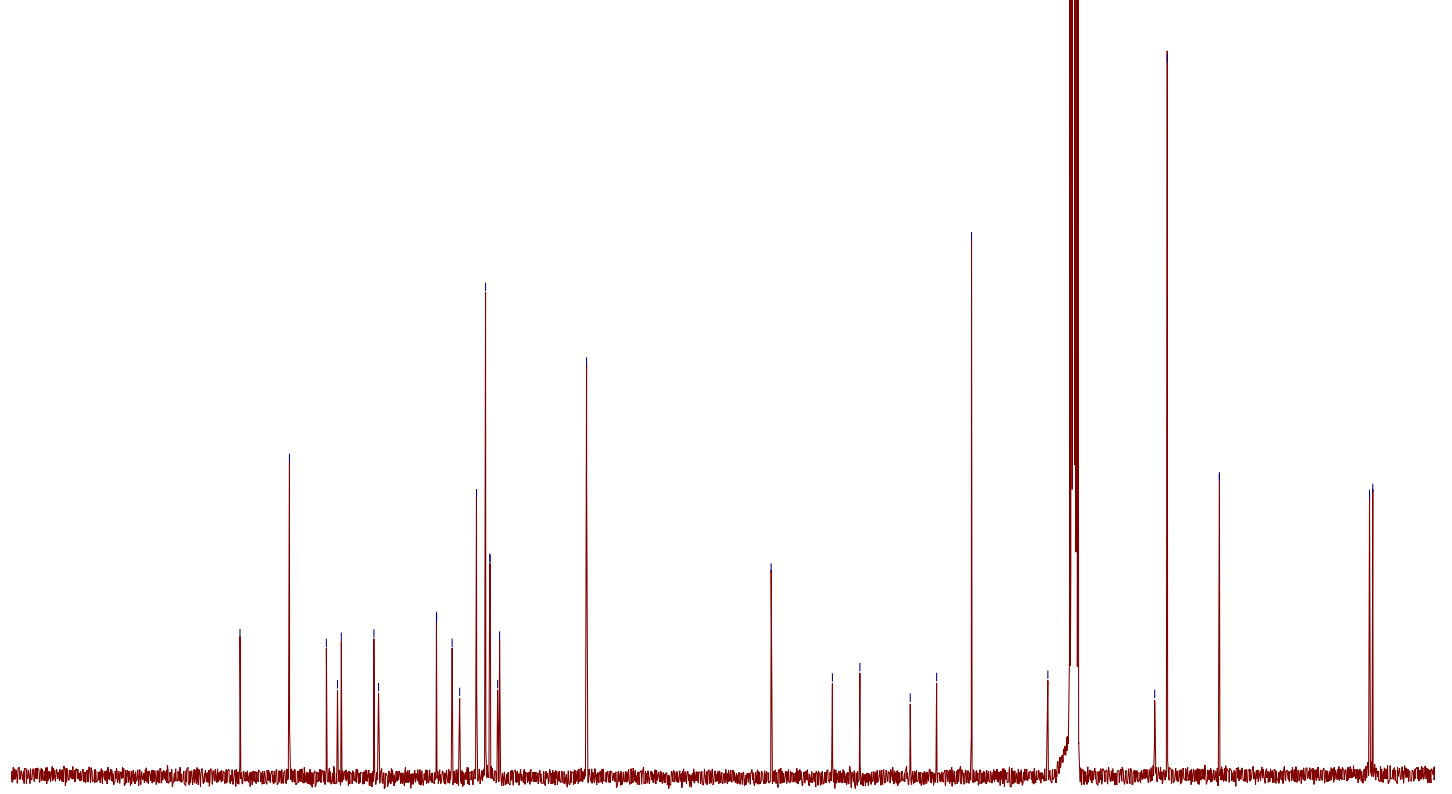

\begin{tabular}{llllllllllllllllllllllll}
\hline 00 & 190 & 180 & 170 & 160 & 150 & 140 & 130 & 120 & 110 & 100 & 90 & 80 & 70 & 60 & 50 & 40 & 30 & 20 & 10 & 0 & -10
\end{tabular} 
${ }^{1} \mathrm{H}$ NMR spectrum $\left(500 \mathrm{MHz}\right.$ ) of compound 5 in $\mathrm{CD}_{3} \mathrm{CN}$

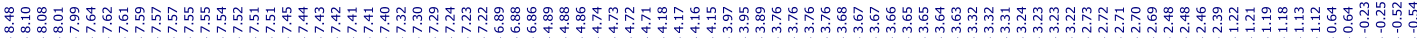

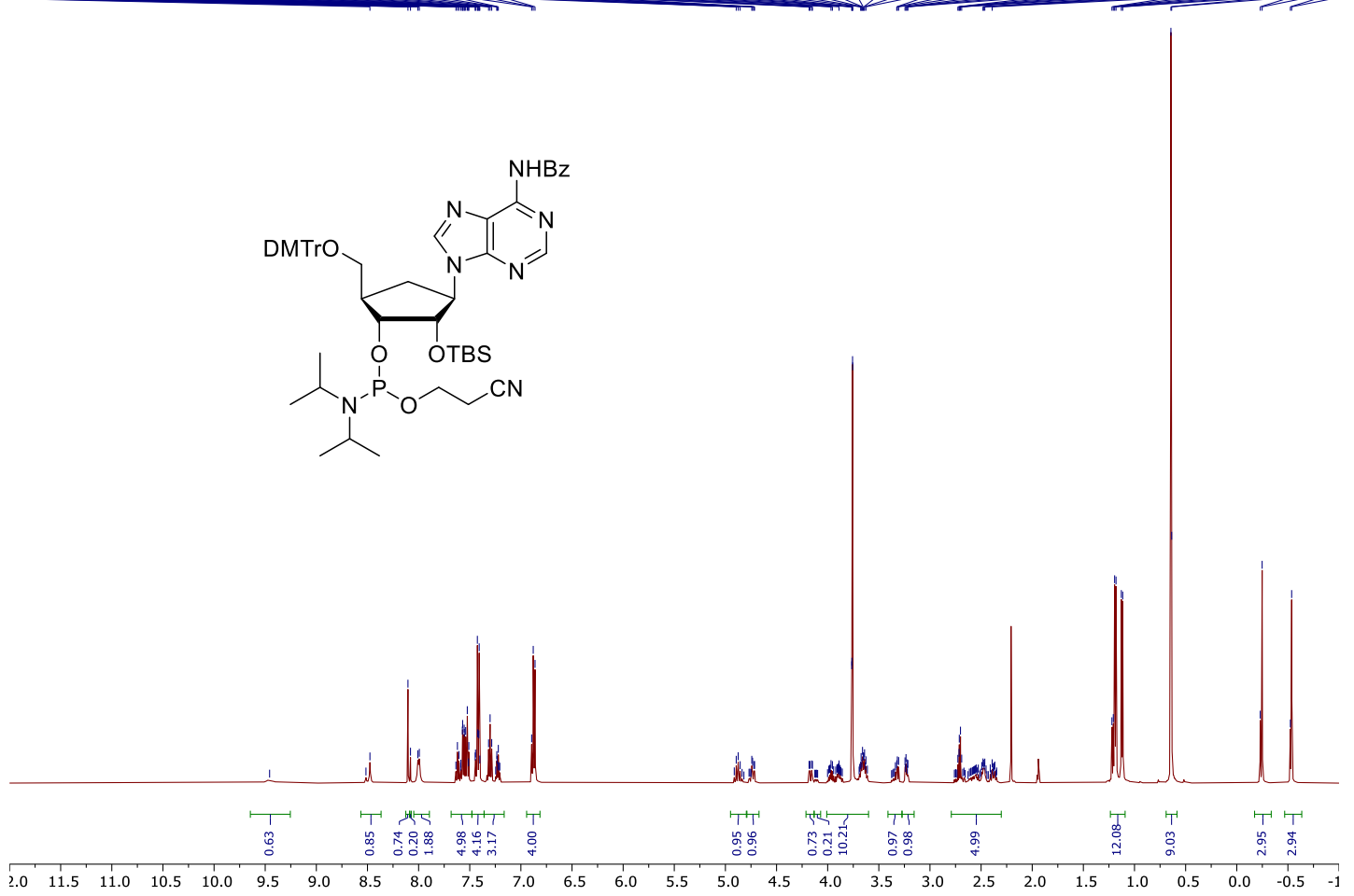

${ }^{13} \mathrm{C}$ NMR spectrum (101 MHz) of compound 5 in $\mathrm{CD}_{3} \mathrm{CN}$

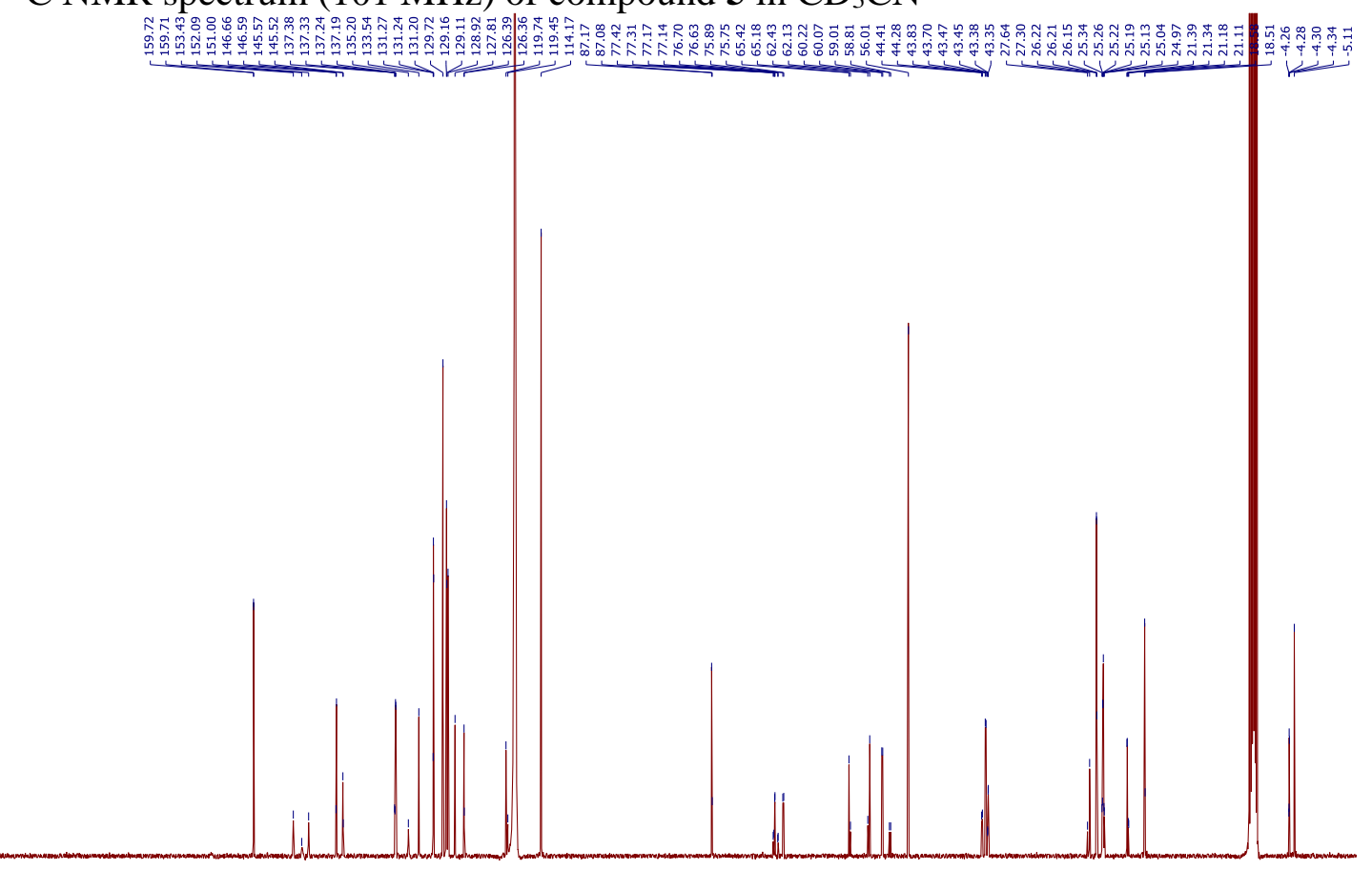


${ }^{31} \mathrm{P}$ NMR spectrum $\left(202 \mathrm{MHz}\right.$ ) of compound $\mathbf{5}$ in $\mathrm{CD}_{3} \mathrm{CN}$

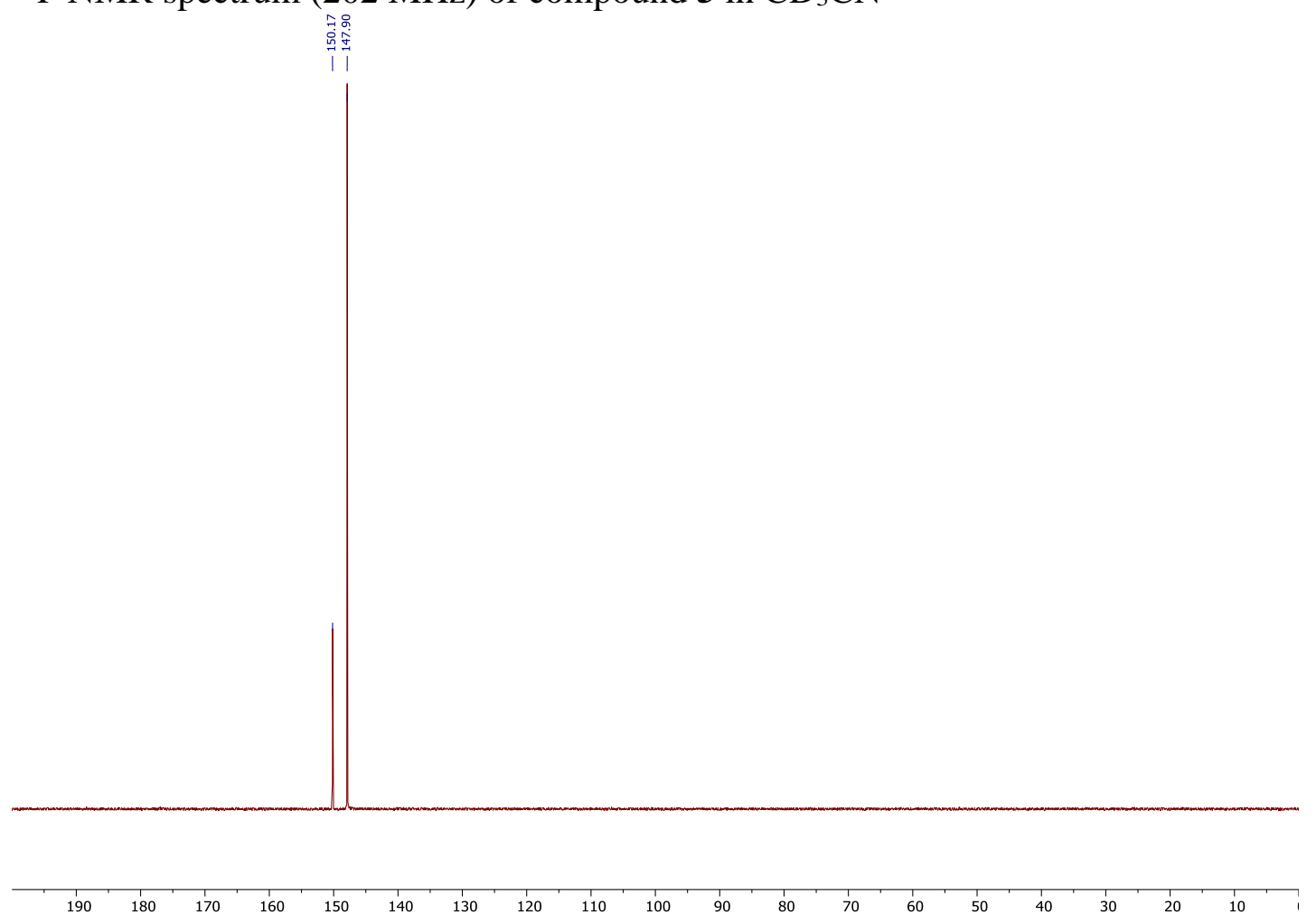


${ }^{1} \mathrm{H}$ NMR spectrum $(500 \mathrm{MHz})$ of compound 30 in DMSO- $d_{6}$

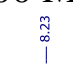

密
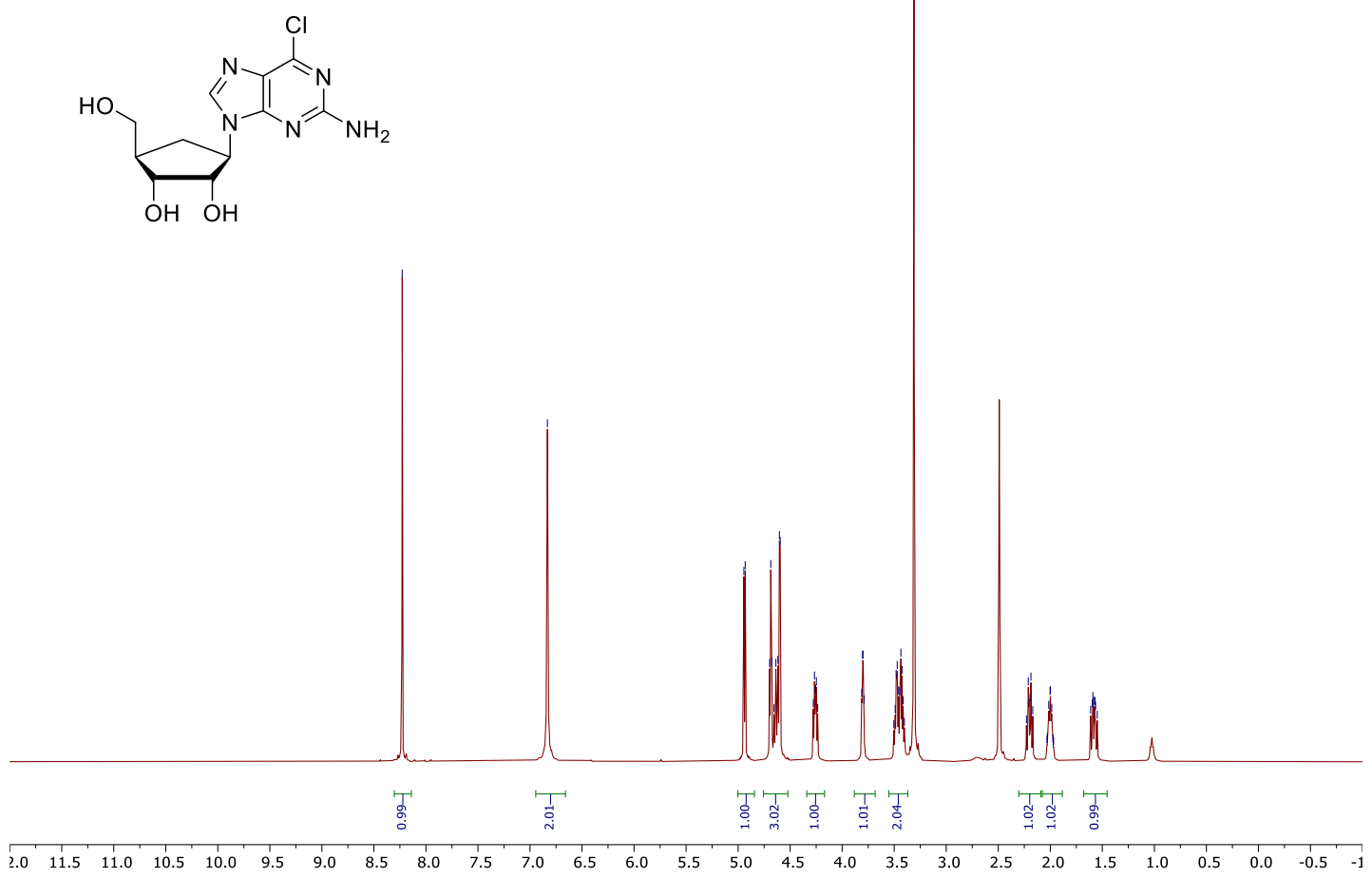

${ }^{13} \mathrm{C}$ NMR spectrum (101 MHz) of compound 30 in DMSO- $d_{6}$

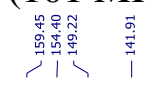

:

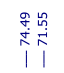

齐

$\stackrel{\text { a }}{\stackrel{9}{1}}$
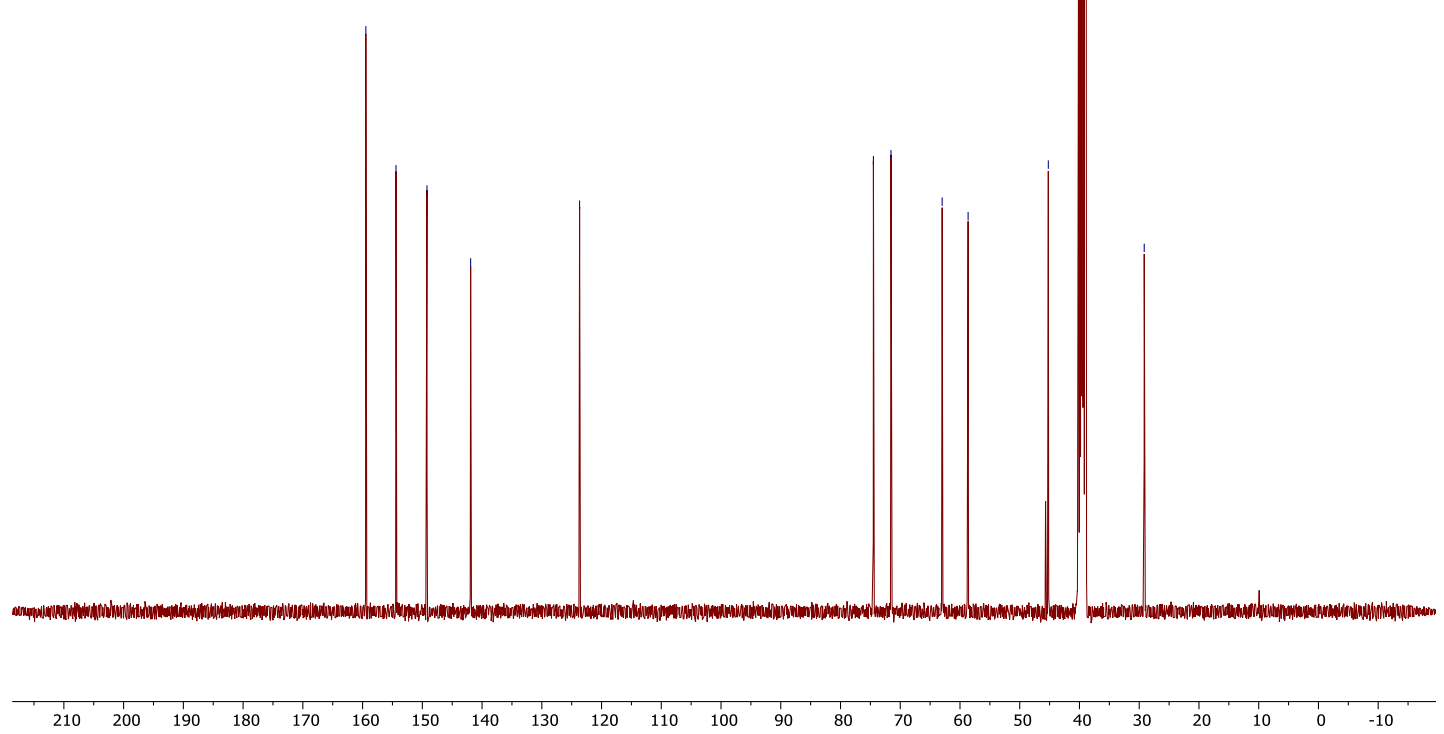

- S61 - 
${ }^{1} \mathrm{H}$ NMR spectrum (500 MHz) of compound 31 in DMSO- $d_{6}$
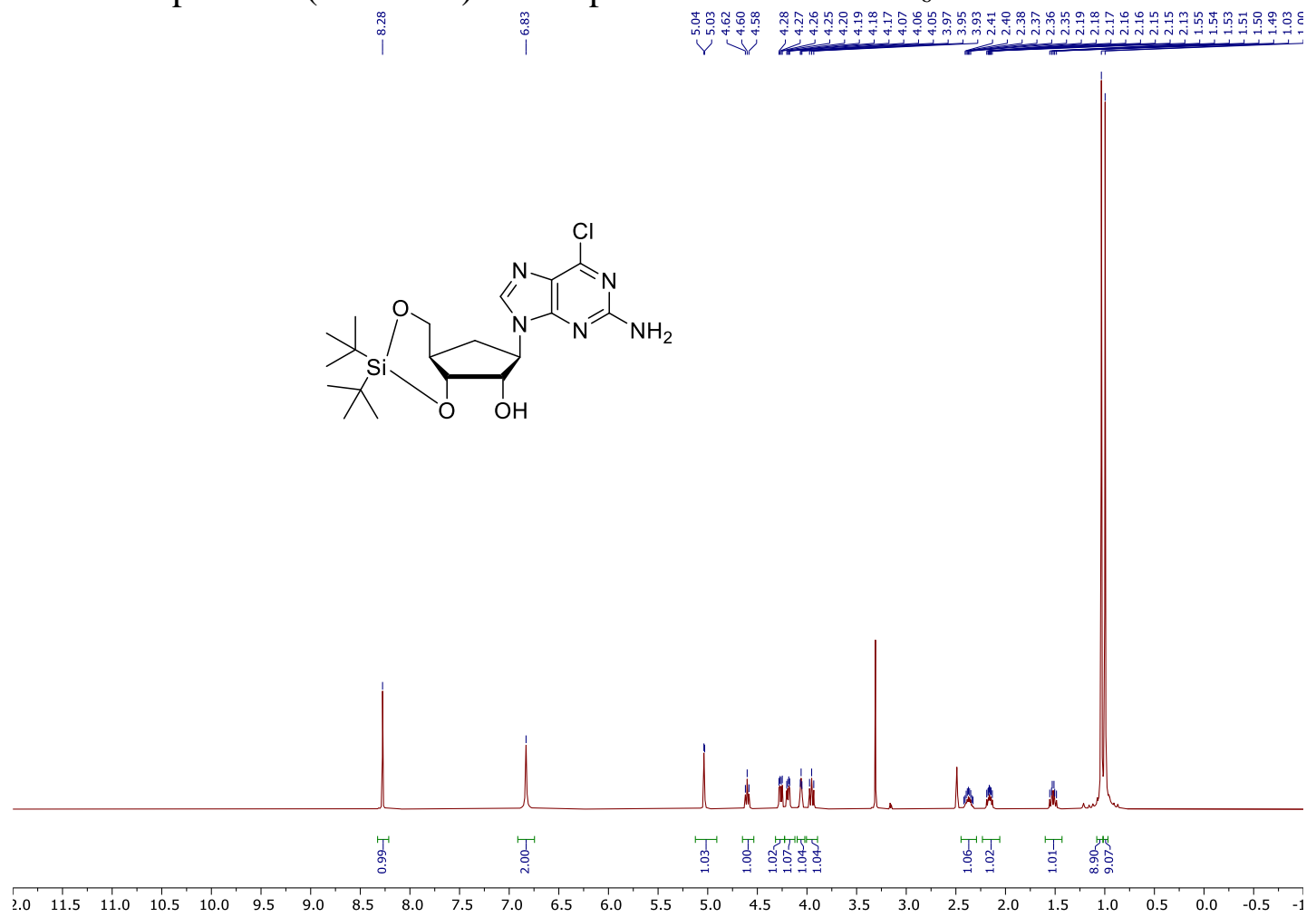

${ }^{13} \mathrm{C}$ NMR spectrum (126 MHz) of compound 31 in DMSO- $d_{6}$
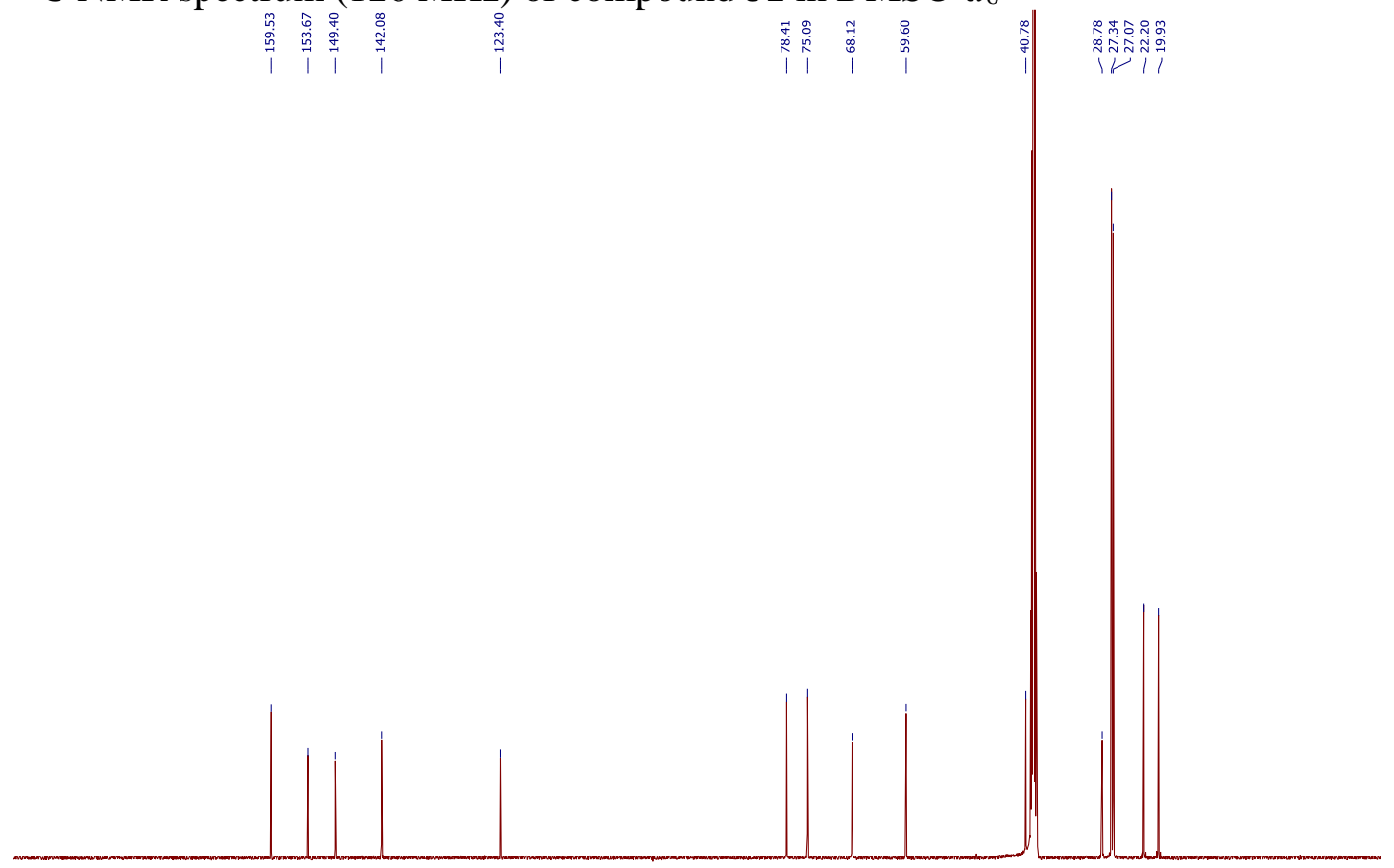

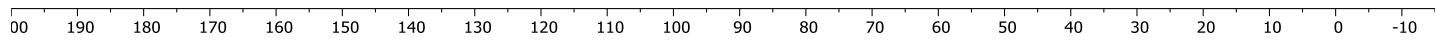


${ }^{1} \mathrm{H}$ NMR spectrum (400 MHz) of compound 32 in DMSO- $d_{6}$
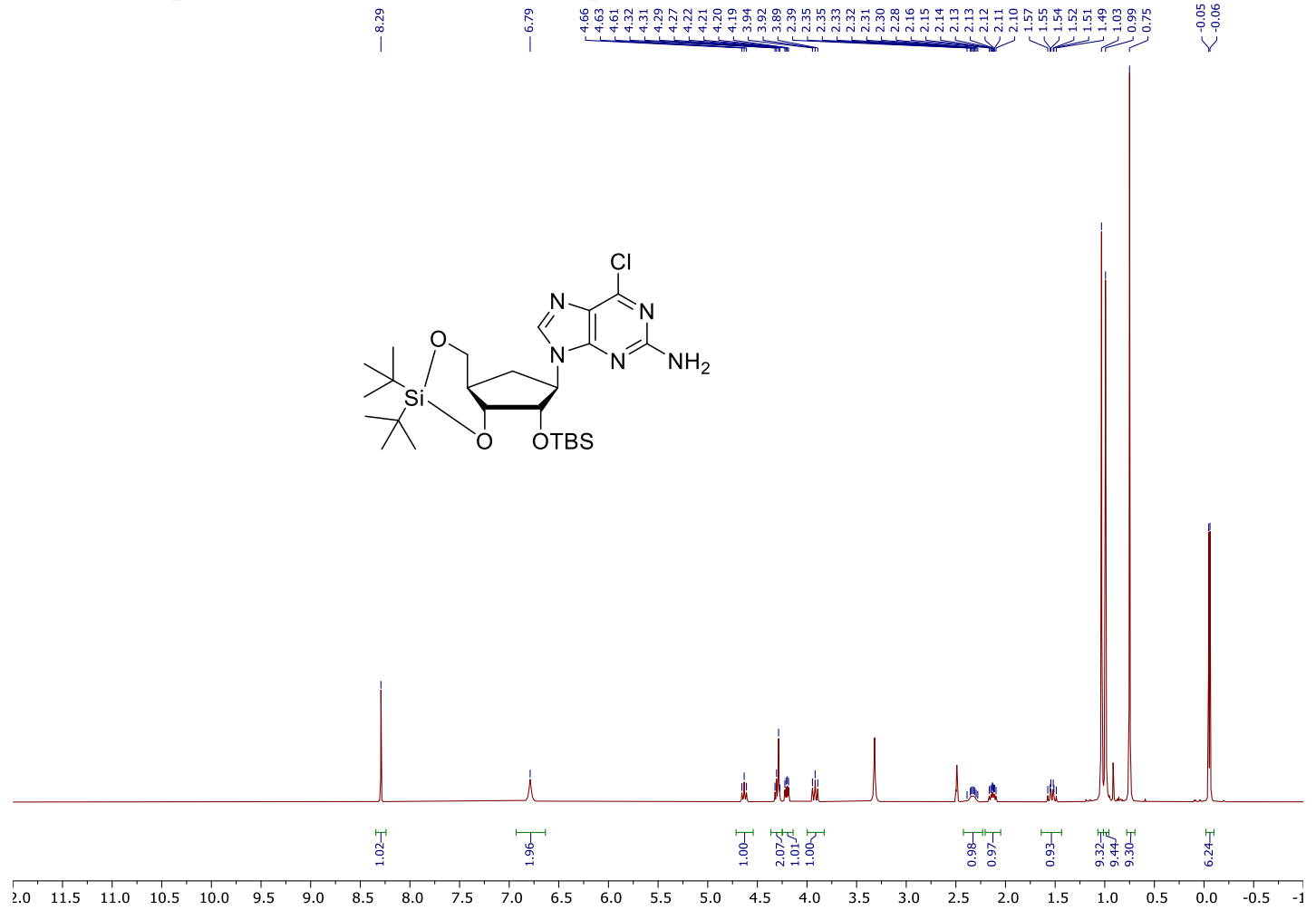

${ }^{13} \mathrm{C}$ NMR spectrum (126 MHz) of compound 32 in DMSO- $d_{6}$
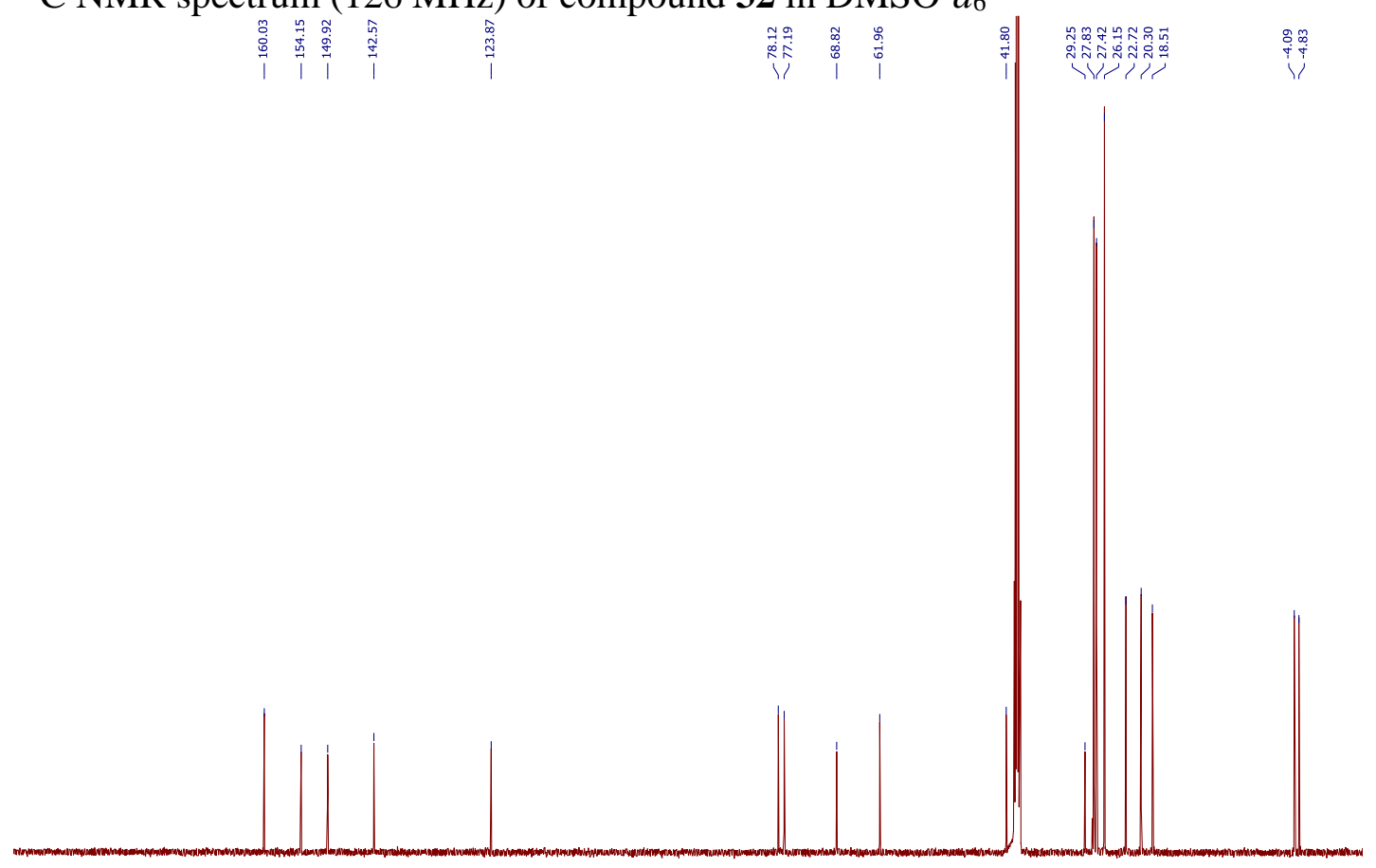

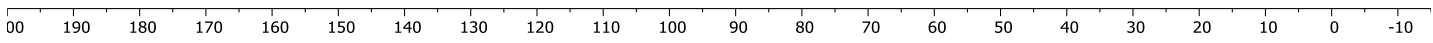


${ }^{1} \mathrm{H}$ NMR spectrum $(500 \mathrm{MHz})$ of compound 33 in DMSO- $d_{6}$

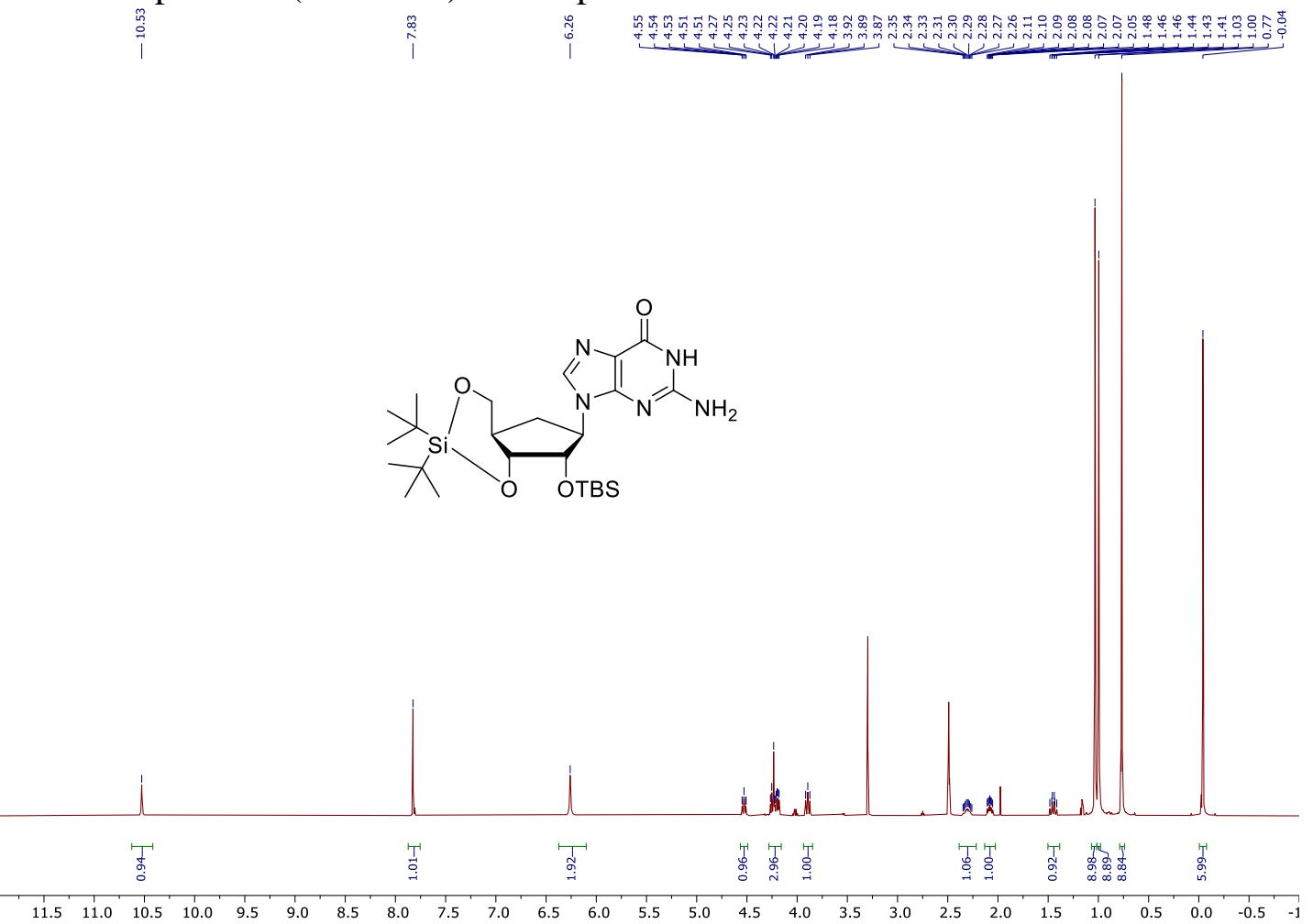

${ }^{13} \mathrm{C}$ NMR spectrum (126 MHz) of compound 33 in DMSO- $d_{6}$

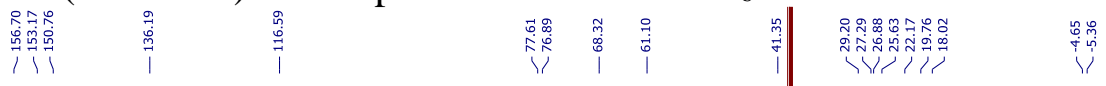

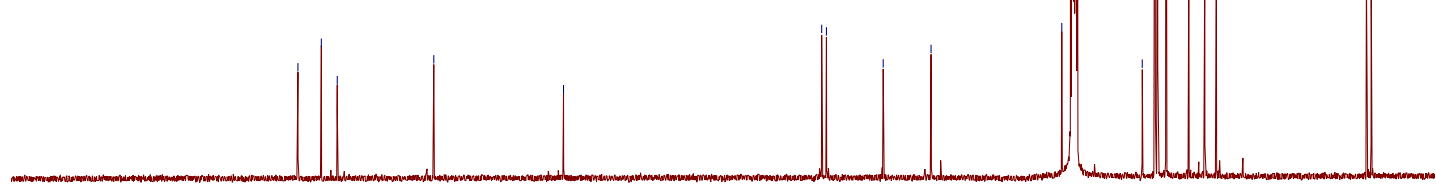

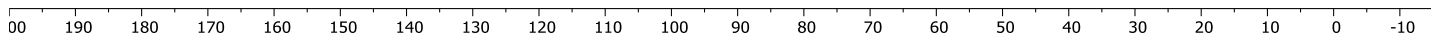


${ }^{1} \mathrm{H}$ NMR spectrum $(500 \mathrm{MHz})$ of compound 34 in DMSO- $d_{6}$

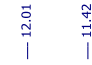

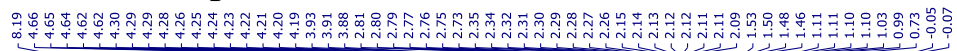
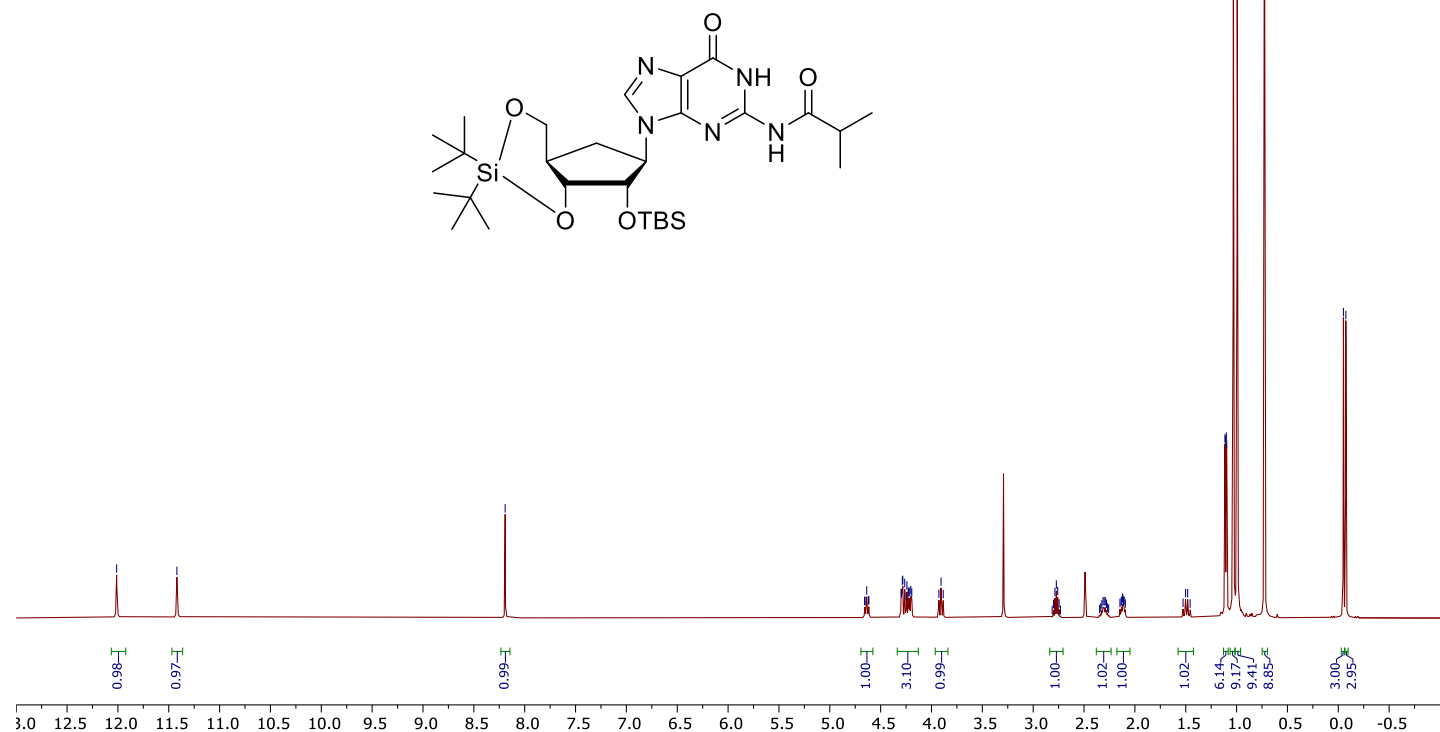

${ }^{13} \mathrm{C}$ NMR spectrum (126 MHz) of compound 34 in DMSO- $d_{6}$

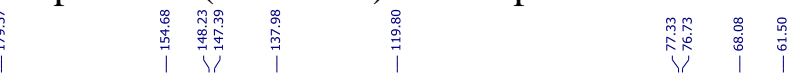

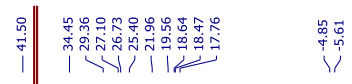

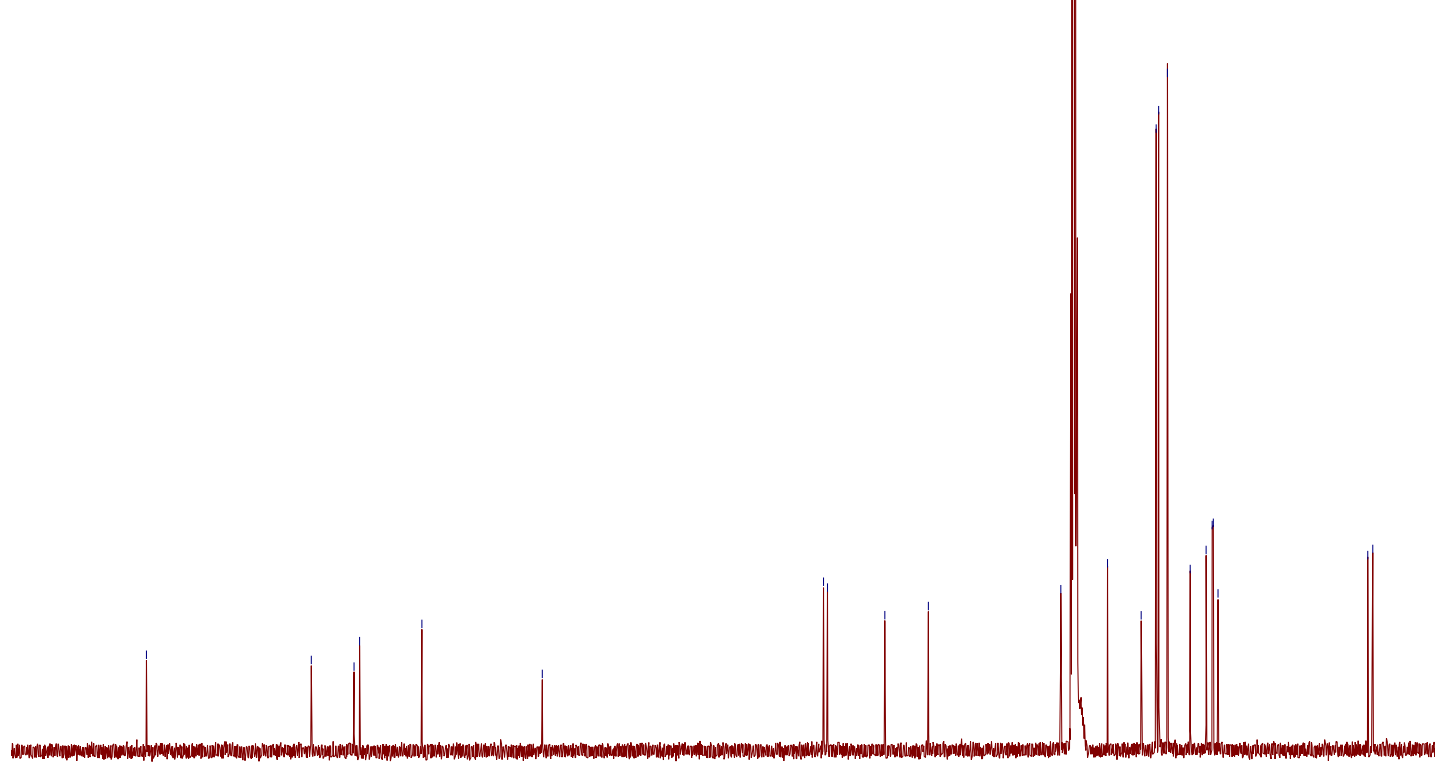

\begin{tabular}{lllllllllllllllllllllll}
\hline 00 & 190 & 180 & 170 & 160 & 150 & 140 & 130 & 120 & 110 & 100 & 90 & 80 & 70 & 60 & 50 & 40 & 30 & 20 & 10 & 0 & -10
\end{tabular} 
${ }^{1} \mathrm{H}$ NMR spectrum $(500 \mathrm{MHz})$ of compound 35 in DMSO- $d_{6}$
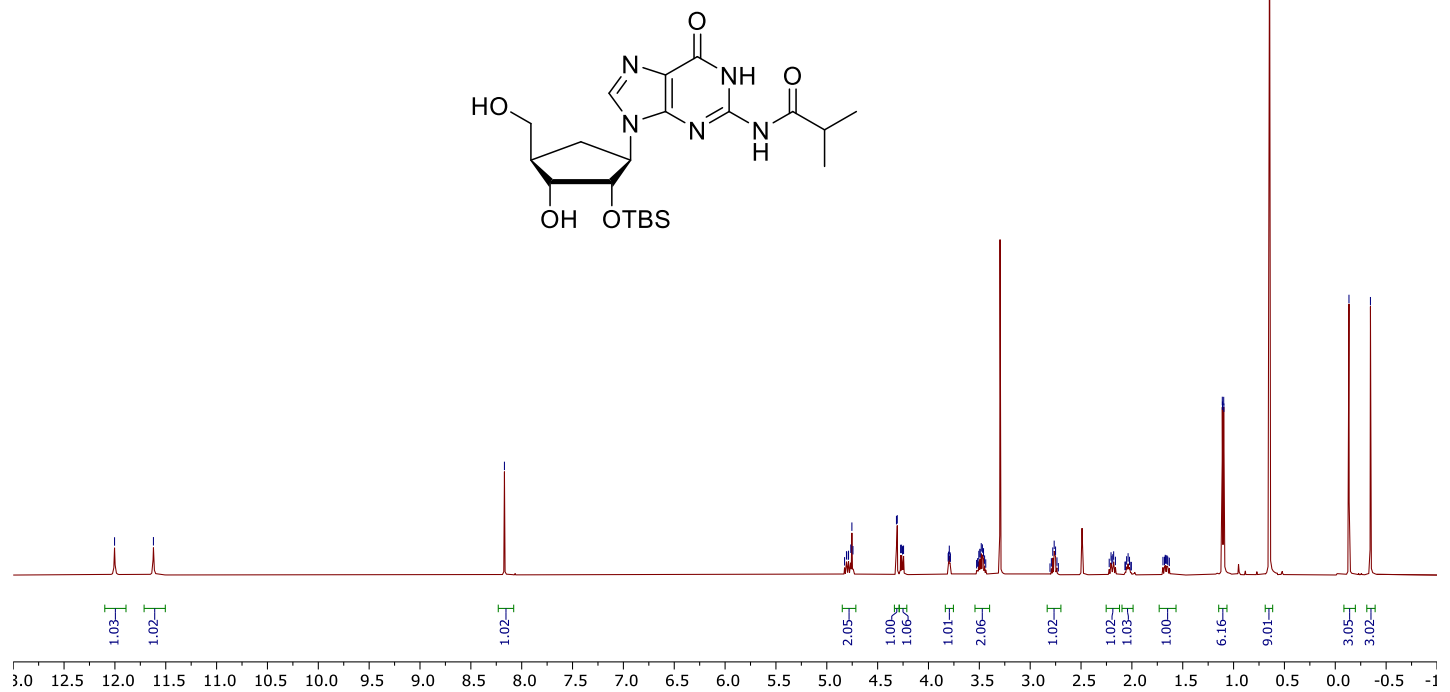

${ }^{13} \mathrm{C}$ NMR spectrum (126 MHz) of compound 35 in DMSO- $d_{6}$

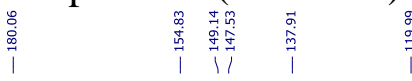
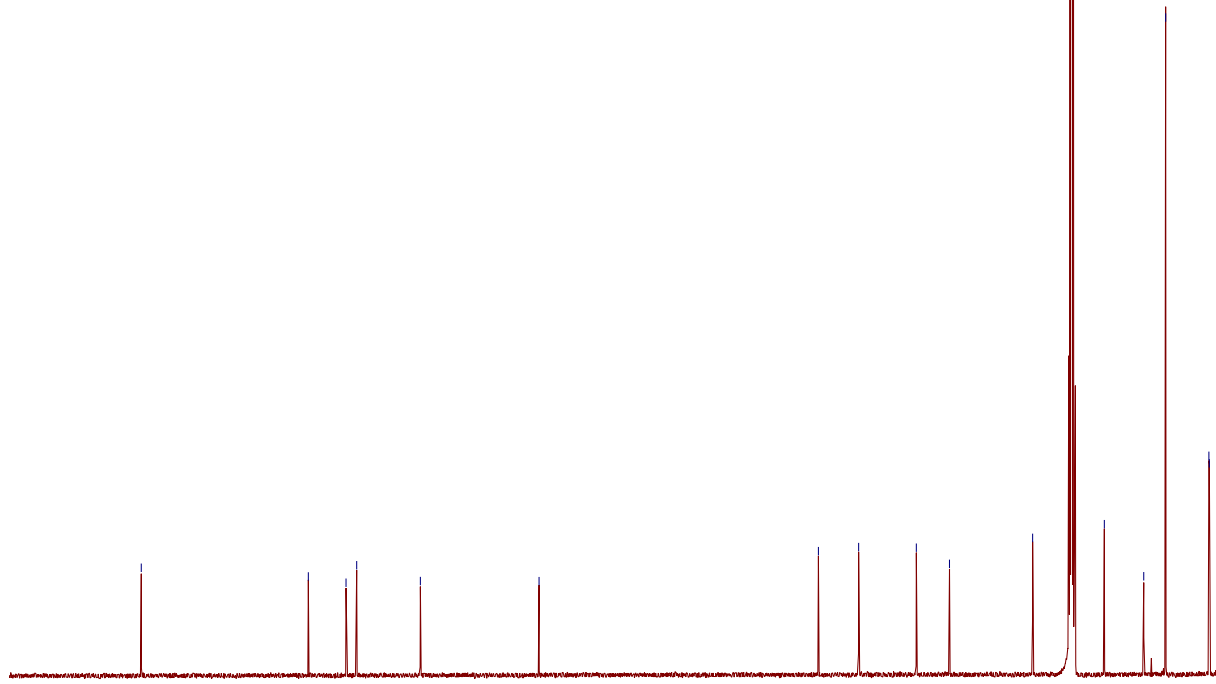

00
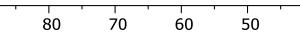
${ }^{1} \mathrm{H}-{ }^{1} \mathrm{H}$ COSY spectrum of compound 35 in DMSO- $d_{6}$

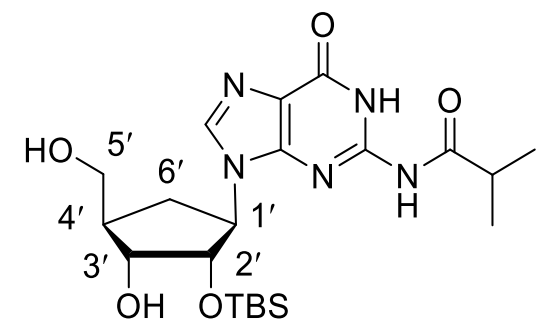

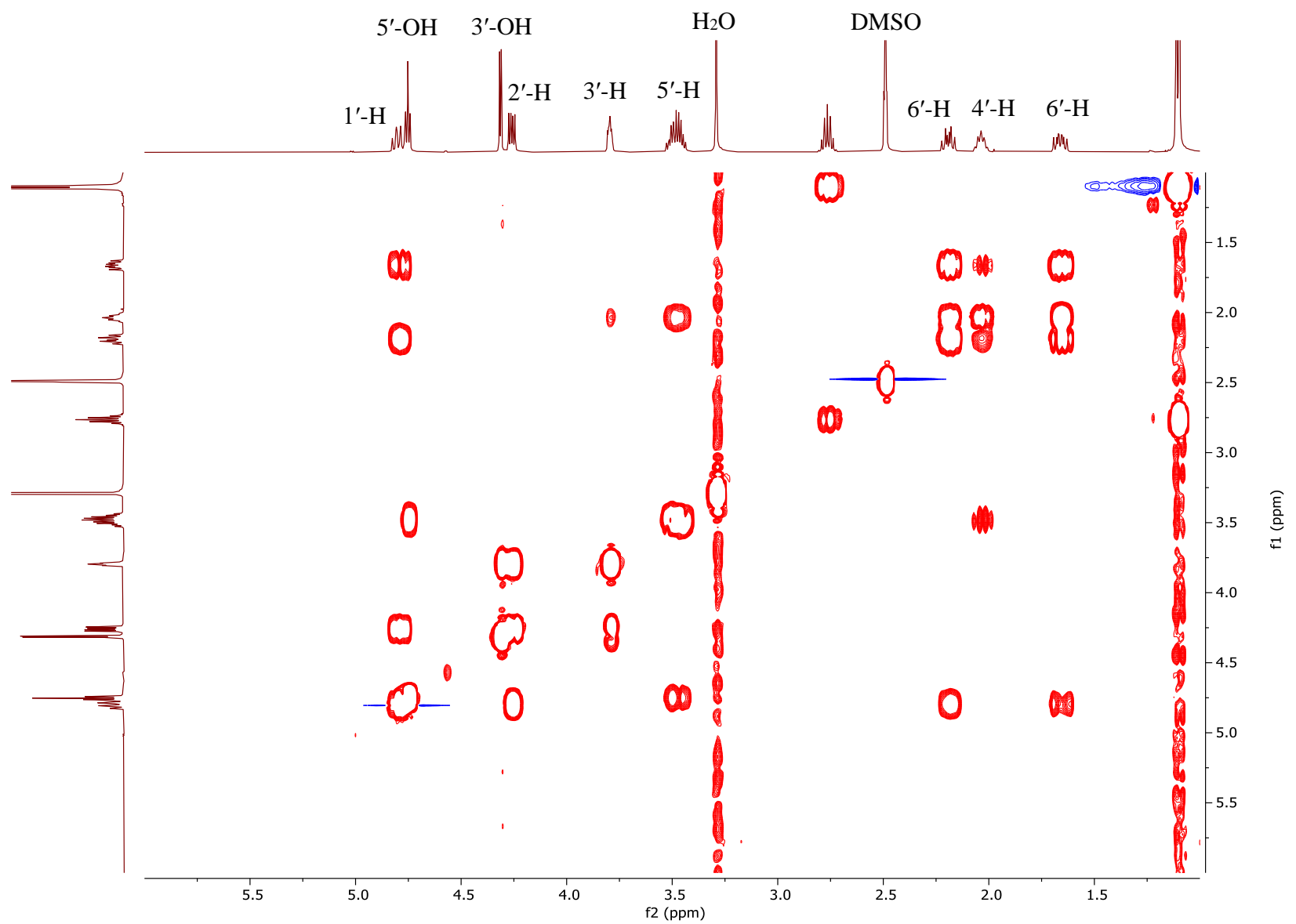


${ }^{1} \mathrm{H}$ NMR spectrum $(500 \mathrm{MHz})$ of compound 36 in DMSO- $d_{6}$

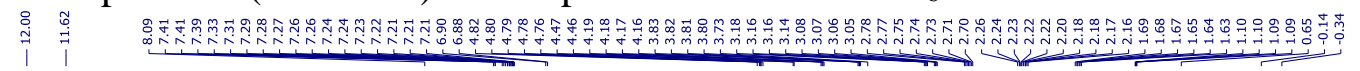

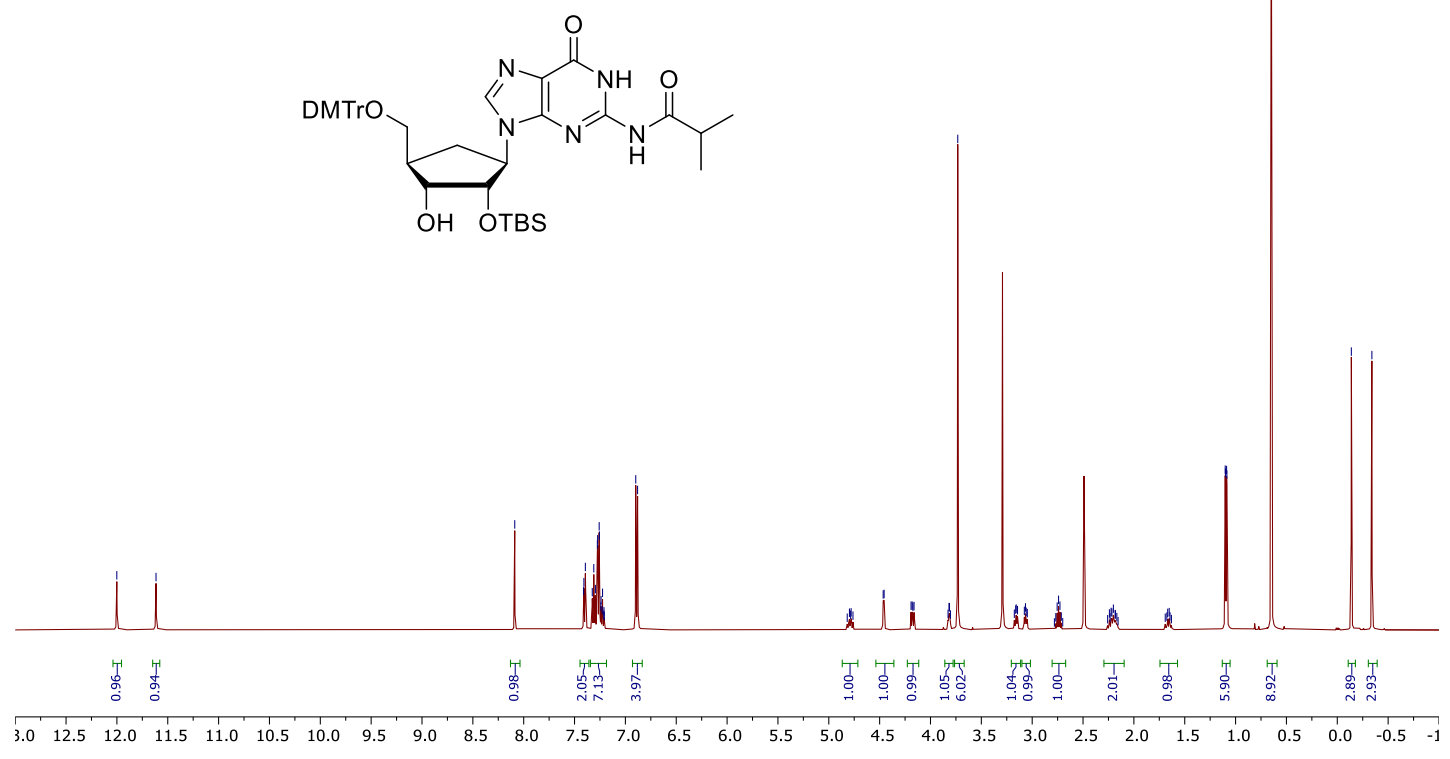

${ }^{13} \mathrm{C}$ NMR spectrum (126 MHz) of compound 36 in DMSO-d $d_{6}$

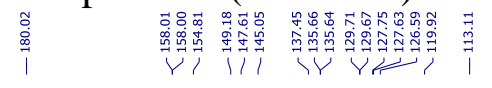

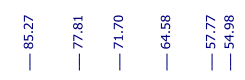

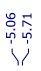
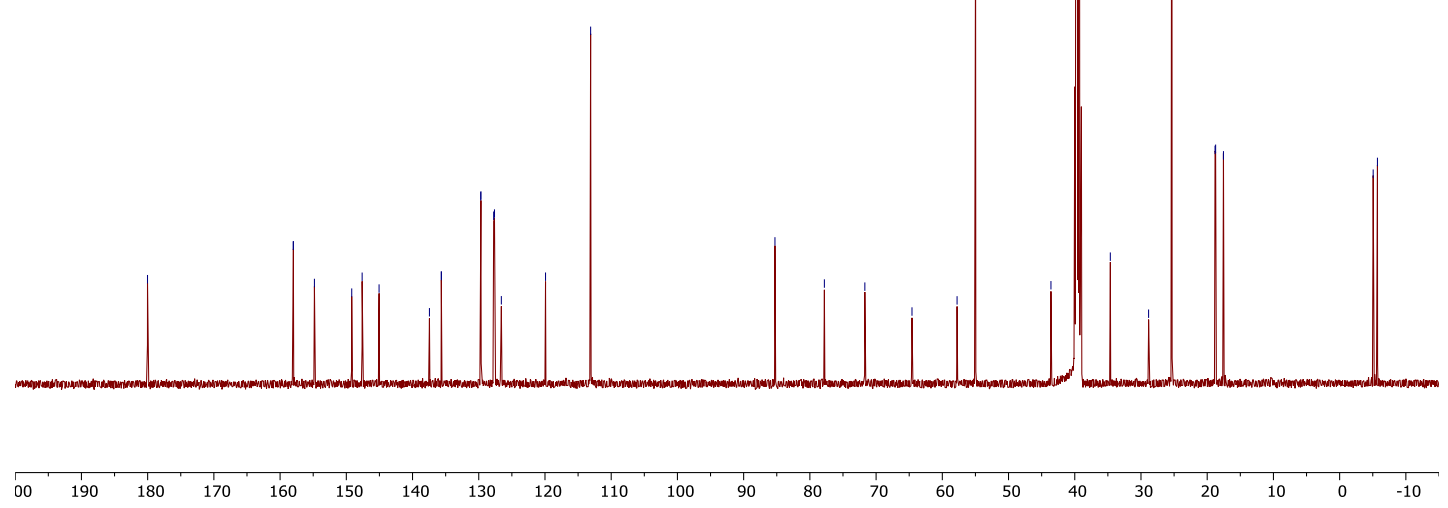

- S68 - 
${ }^{1} \mathrm{H}$ NMR spectrum $(500 \mathrm{MHz})$ of compound 6 in $\mathrm{CD}_{3} \mathrm{CN}$

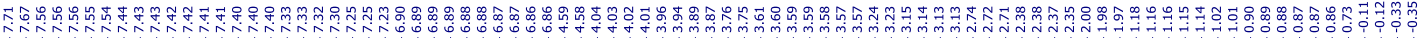

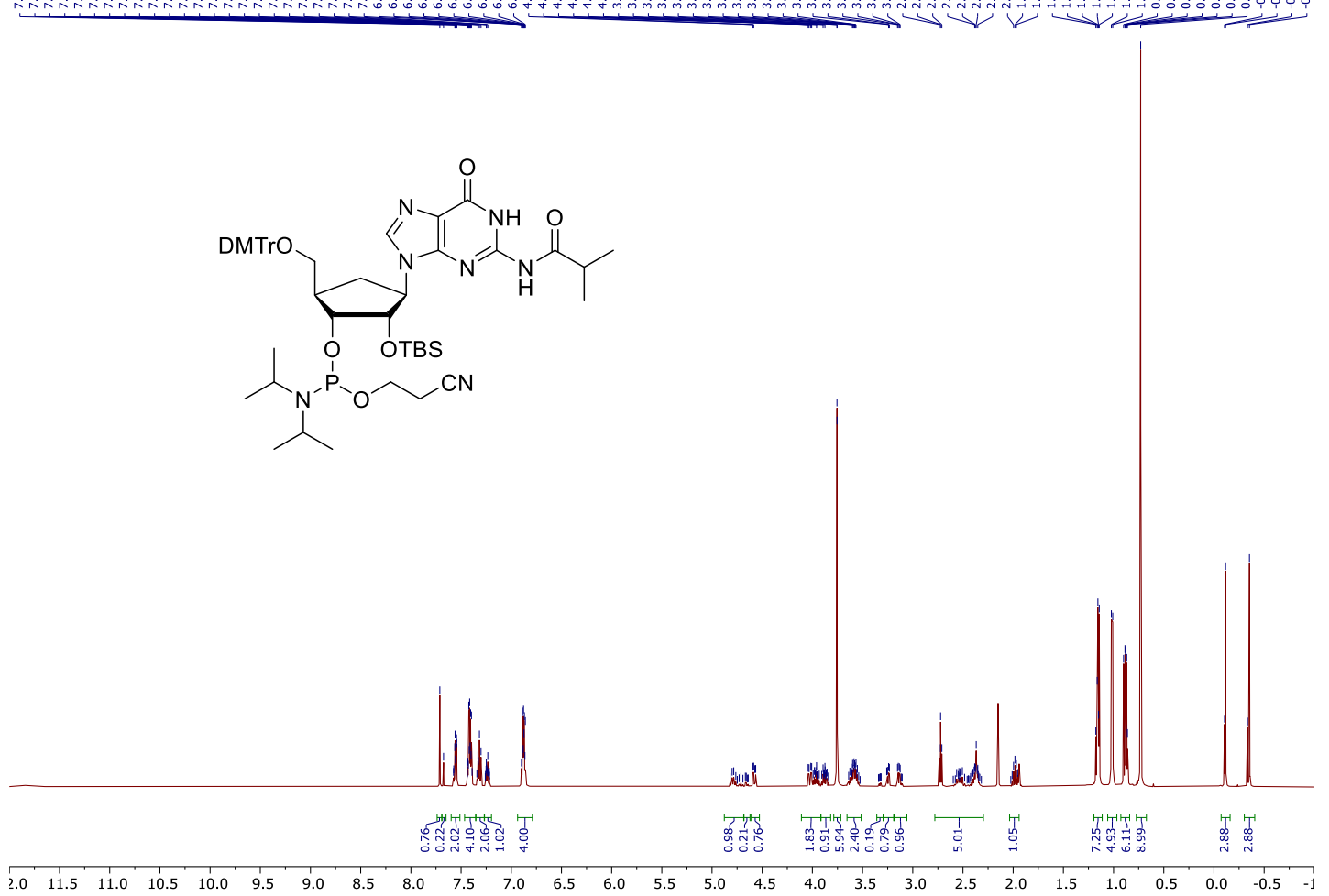

${ }^{13} \mathrm{C}$ NMR spectrum (126 MHz) of compound 6 in $\mathrm{CD}_{3} \mathrm{CN}$

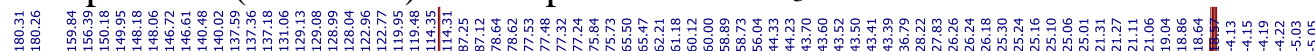

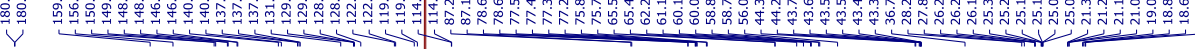

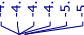

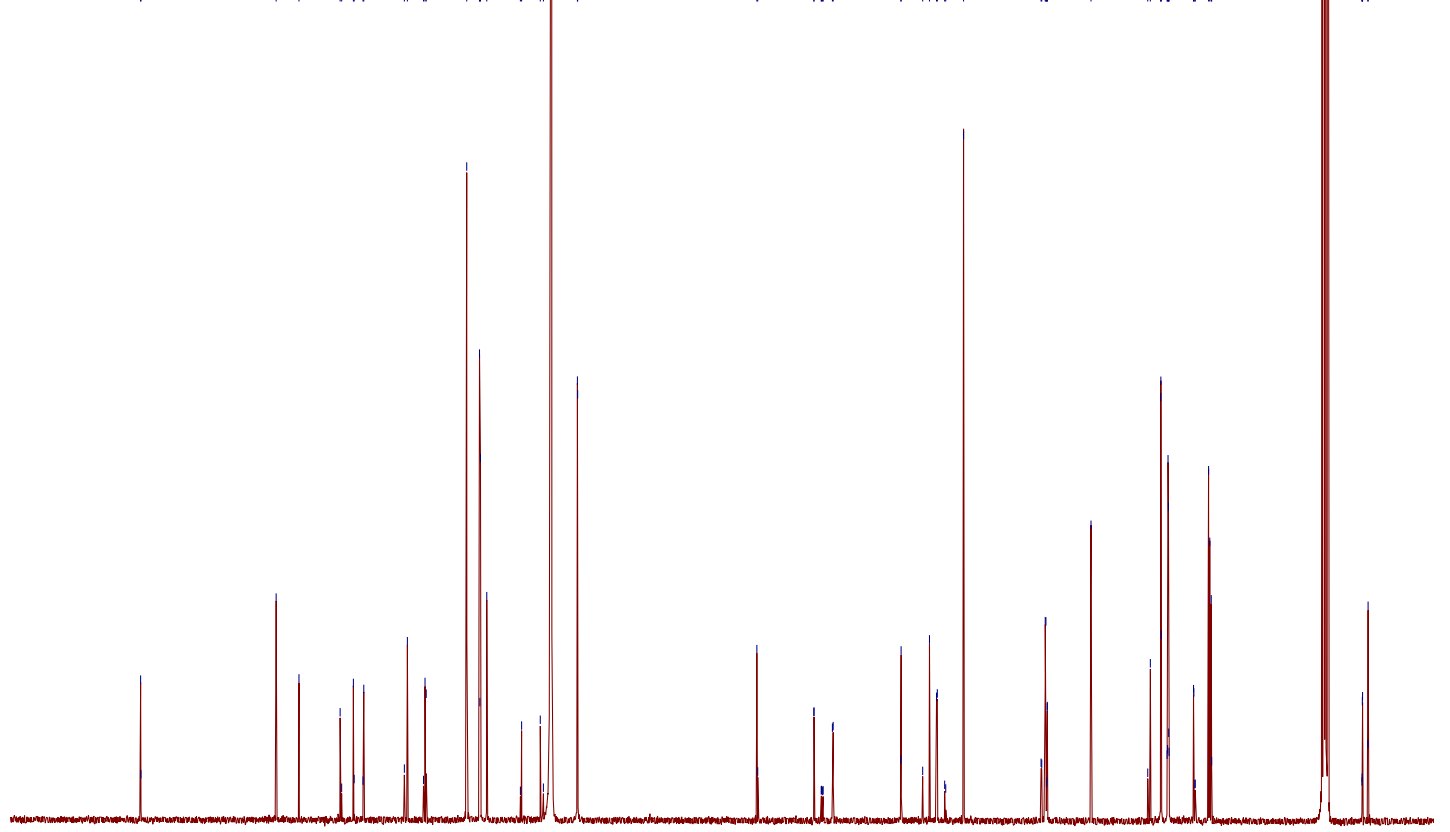

\begin{tabular}{llllllllllllllllllllllll}
\hline 00 & 190 & 180 & 170 & 160 & 150 & 140 & 130 & 120 & 110 & 100 & 90 & 80 & 70 & 60 & 50 & 40 & 30 & 20 & 10 & 0 & -10
\end{tabular} 
${ }^{31} \mathrm{P}$ NMR spectrum $(202 \mathrm{MHz})$ of compound 6 in $\mathrm{CD}_{3} \mathrm{CN}$

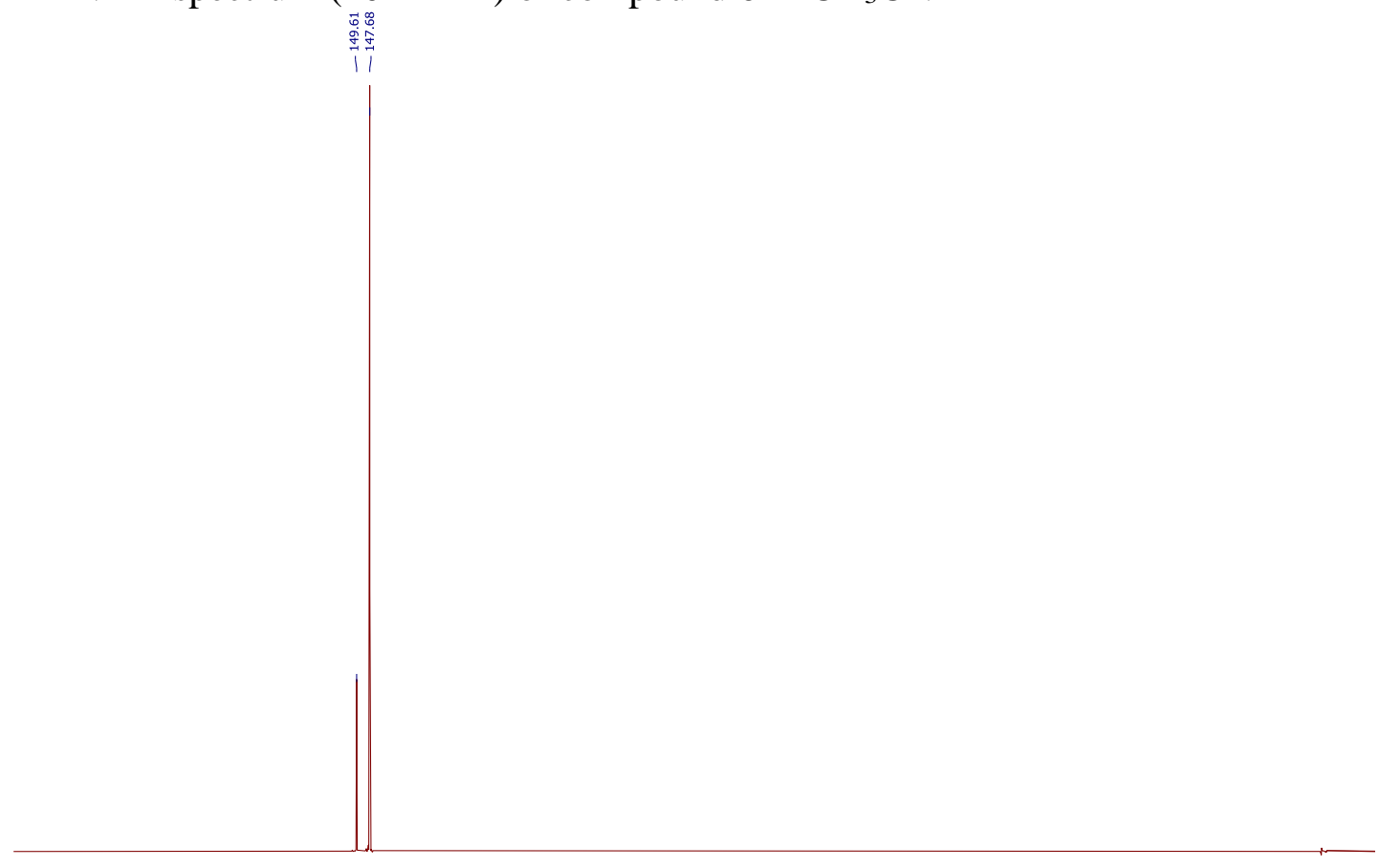

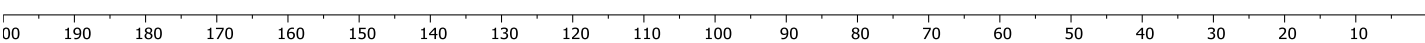




\section{X-ray crystallographic analysis}

Table S2. Refinement statistics

\begin{tabular}{|c|c|c|}
\hline & $\sum_{\mathrm{OH} \mathrm{OH}_{1}}^{\mathrm{Cl}}$ & $x^{l} \sum_{\mathrm{O}}^{\mathrm{Si}}$ \\
\hline Compound & 23 & 31 \\
\hline $\mathrm{CCDC}$ & 2082820 & 2082823 \\
\hline Space group & $P 2_{1} 2_{1} 2_{1}$ with $2 \mathrm{~mol} / \mathrm{asu}$ & $C 222_{1}$ with $1 \mathrm{~mol} / \mathrm{asu}$ \\
\hline \multicolumn{3}{|l|}{ Cell } \\
\hline $\mathrm{a}(\AA)$ & $6.6433(4)$ & $6.801(4)$ \\
\hline b $(\AA)$ & $15.0052(9)$ & $15.506(9)$ \\
\hline c $(\AA)$ & $43.528(2)$ & $23.522(13)$ \\
\hline Resolution $(\AA)$ & 0.70 & 0.68 \\
\hline R1 (\#reflections) & $0.0390(5789)$ & $0.0371(7012)$ \\
\hline wR2 & 0.0956 & 0.0929 \\
\hline Goodness of Fit (S) & 1.044 & 1.035 \\
\hline Flack parameter (classic) & $0.025(16)$ & $0.012(22)$ \\
\hline Flack parameter (Parsons) & $0.033(7)$ & $0.021(9)$ \\
\hline
\end{tabular}

\section{Crystallization}

Crystals of 23 were grown by dissolving dry compound in ethanol and allowing the ethanol to slowly evaporate. Crystals of $\mathbf{3 1}$ were grown by dissolving dry compound in tetrahydrofuran and equilibrating the solution by vapor diffusion against cyclohexane. Crystals were mounted on Mitegen loops after transferring into Paratone oil.

\section{$\mathrm{X}$-ray diffraction data collection and structure solution and refinement}

X-ray diffraction data were collected using the D8 Venture (Bruker AXS, Madison, WI) system in the Biomolecular Crystallography Facility in the Vanderbilt University Center for Structural Biology. The system includes an Excillum D2+ MetalJet X-ray source with Helios MX optics providing $\mathrm{Ga} \mathrm{K} \alpha$ radiation at $1.3418 \AA$ wavelength. The crystal was mounted on a kappa axis goniometer and maintained at $100 \mathrm{~K}$ using an Oxford Cryosystems Cryostream 800 cryostat. The detector was a PHOTON III charge-integrating pixel array detector. Data collection was performed in shutterless mode. Data were reduced using APEX3 software (Bruker AXS, Madison, WI) employing SAINT v8.40A (Bruker Nano, Inc., 2019) as well as SADABS ${ }^{4}$ for multi-scan absorbance correction.

Structures were solved using SHELXT 2014/5 5 and refined using SHELXL-2018/3 ${ }^{6}$. Absolute structure was determined using the Flack parameter. ${ }^{7}$ The structures were viewed using shelXle. ${ }^{8}$ 


\section{Oligonucleotide synthesis and characterization}

Oligonucleotides were synthesized on a MerMade 192 oligonucleotide synthesizer using standard

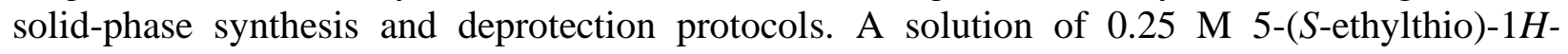
tetrazole in acetonitrile $\left(\mathrm{CH}_{3} \mathrm{CN}\right)$ was used as the activator. The phosphoramidite solutions (standard RNA and DNA amidites and synthesized car-RNA amidites) were prepared at $0.100 \mathrm{M}$ in anhydrous $\mathrm{CH}_{3} \mathrm{CN}$. The oxidizing reagent was $0.02 \mathrm{M} \mathrm{I}_{2}$ in $\mathrm{THF} /$ pyridine $/ \mathrm{H}_{2} \mathrm{O}$. The detritylation reagent was $3 \%$ dichloroacetic acid (DCA) in $\mathrm{CH}_{2} \mathrm{Cl}_{2}$. After completion of the automated synthesis, the oligonucleotides were manually released from support and deprotected using $40 \%$ aqueous methylamine and then stored at room temperature shaking for one hour. In the case of RNA, the 2'-hydroxyl groups were deprotected by treating with $\mathrm{Et}_{3} \mathrm{~N} \cdot 3 \mathrm{HF}$ at $60{ }^{\circ} \mathrm{C}$ for $45 \mathrm{~min}$. Oligonucleotides were purified using anion-exchange high-performance liquid chromatography (Source $^{\mathrm{TM}}$ 15Q 4.6 x $100 \mathrm{~mm}$, Mobile Phase A (20 mM Sodium Phosphate, 15\% $\mathrm{CH}_{3} \mathrm{CN}, 100 \mathrm{mM}$ Urea, $\mathrm{pH}$ 8.5), Mobile Phase B (20mM Sodium Phosphate, $1 \mathrm{M} \mathrm{NaBr}, 15 \% \mathrm{CH}_{3} \mathrm{CN}, 100 \mathrm{mM}$ Urea, $\mathrm{pH} 8.5$ ), linear gradient from A/B 90/10 to $40 / 60$ over $10 \mathrm{mins}$, flow rate $=3.5 \mathrm{~mL} / \mathrm{min}, \mathrm{I}=270$ $\mathrm{nm}, \mathrm{tR}=5-7$ mins) and desalted using size-exclusion chromatography with water as an eluent. Oligonucleotides were then quantified by measuring the absorbance at $260 \mathrm{~nm}$ using the following extinction coefficients: (A, $13.86 \mathrm{~L} / \mathrm{mol} / \mathrm{cm}$; T/U, 7.92 L/mol $/ \mathrm{cm}$; C, $6.57 \mathrm{~L} / \mathrm{mol}^{\prime} \mathrm{cm}$; and G, 10.53 $\mathrm{L} / \mathrm{mol}^{\prime} \mathrm{cm}$ ). The purities and identities of modified oligonucleotides were verified by analytical anion exchange chromatography and mass spectrometry, respectively (Table S3).

Table S3. MS ( $\mathrm{m} / z)$ analysis of modified oligonucleotides ${ }^{a}$

\begin{tabular}{cccc}
\hline & & \multicolumn{2}{c}{ mass $(\mathrm{m} / \mathrm{z})$} \\
Oligonucleotide\# & sequence $\left(5^{\prime}-3^{\prime}\right)$ & calc. & obs. \\
\hline 1 & rUACAGUCUAUGU & 3755.3 & 3755.4 \\
2 & rUACAGUCUAUGU & 3755.3 & 3755.5 \\
3 & rACAUAGACUGUA & 3801.4 & 3801.5 \\
4 & rACAUAGACUGUA & 3801.4 & 3801.7 \\
5 & rUACAGUCUAUGU & 3733.6 & 3733.2 \\
6 & rACAUAGACUGUA & 3779.7 & 3780.2 \\
\hline
\end{tabular}

${ }^{a}$ Prefix $\mathrm{r}$ indicates ribonucleotide, prefix $\mathrm{d}$ indicates deoxyribonucleotide. Bold red letters indicate sites of car-RNA modification. 


\section{MS characterization of modified oligonucleotides}

\section{Oligonucleotide 1}

Deconvoluted Mass Spectrum of A_2749866_3, RT = $0.376 \mathrm{~min}$ :

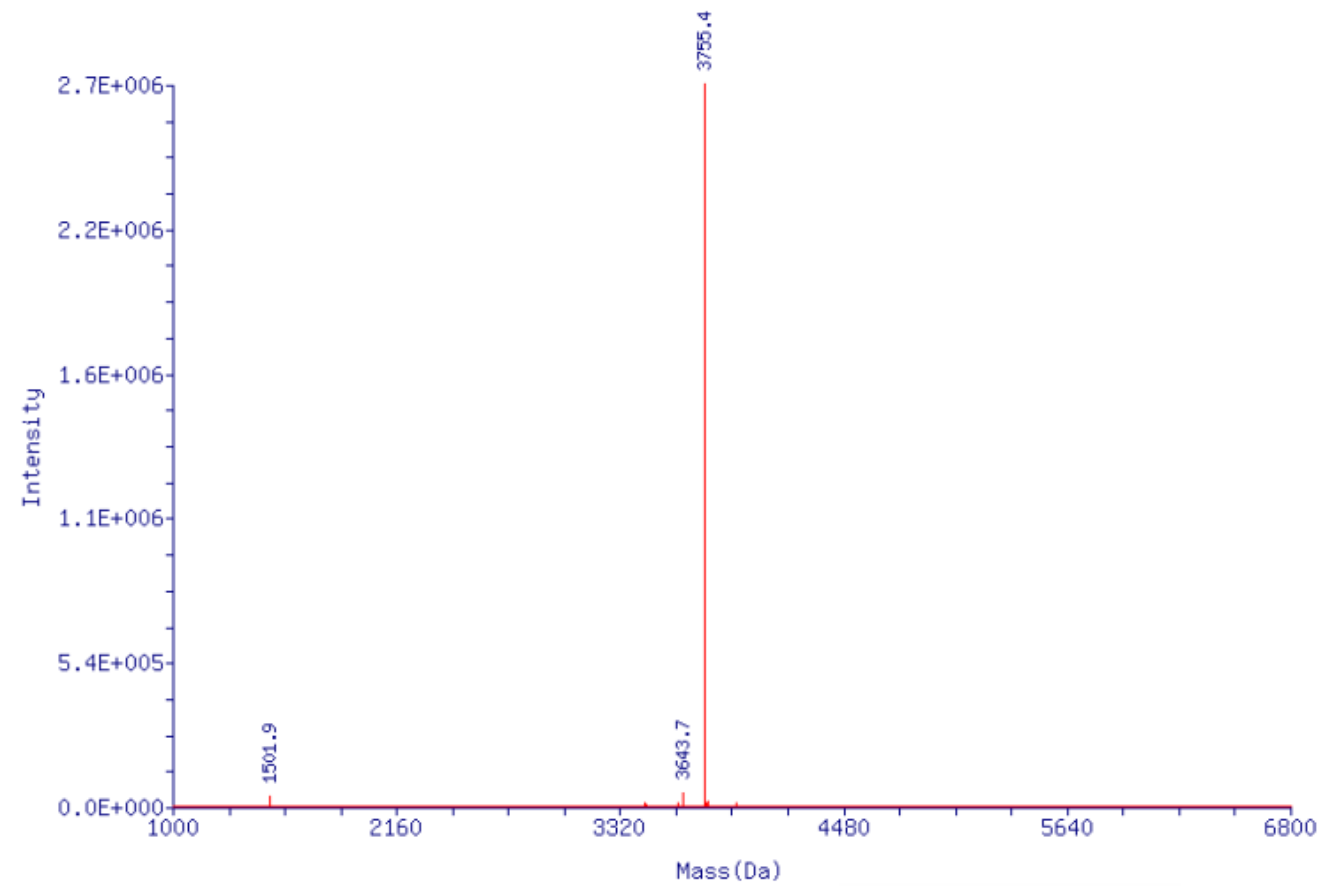

\section{Oligonucleotide 2}

Deconvoluted Mass Spectrum of A_2749864_3, RT = $0.365 \mathrm{~min}$ :

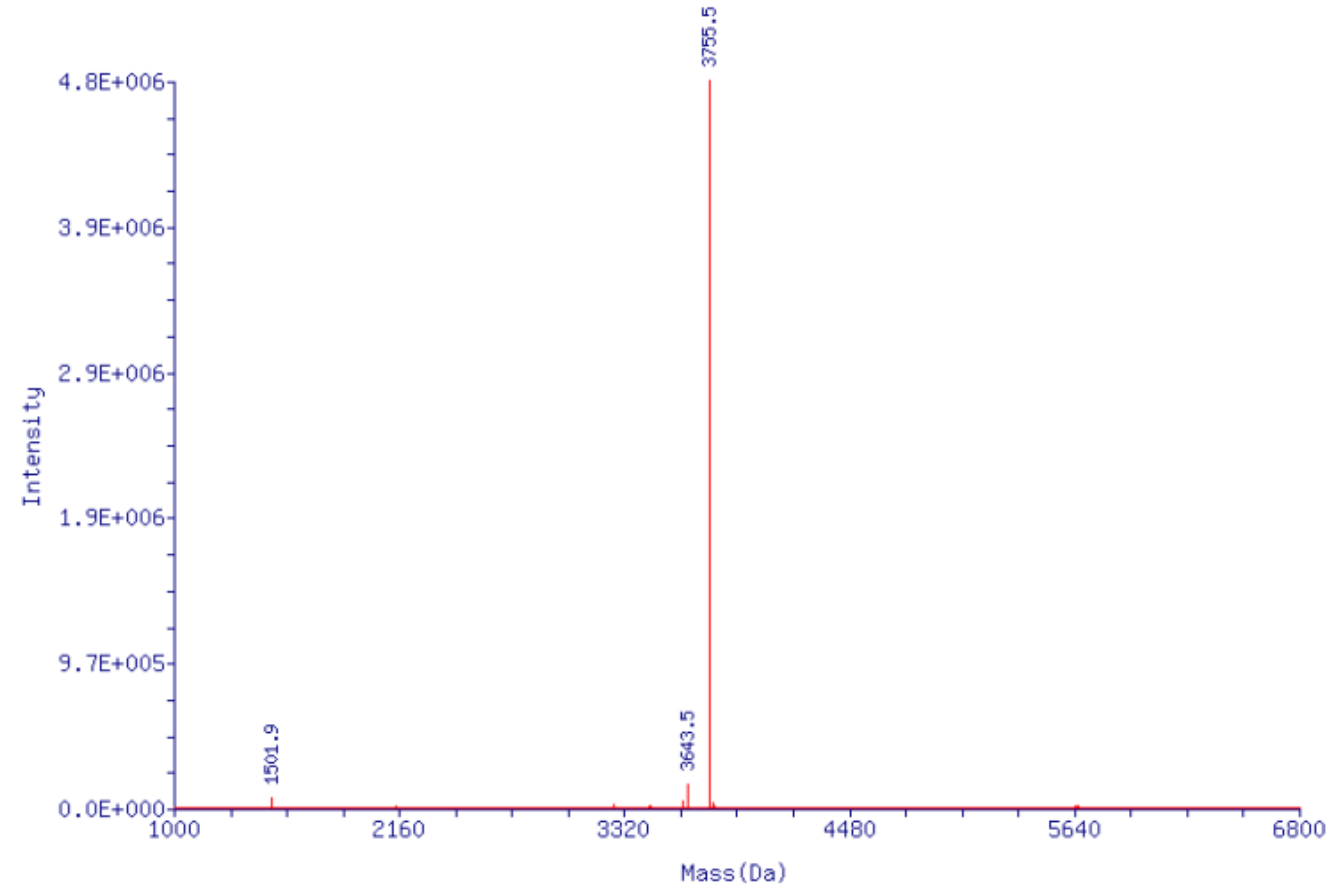




\section{Oligonucleotide 3}

Deconvoluted Mass Spectrum of A_2749863_3, RT = $0.376 \mathrm{~min}$ :

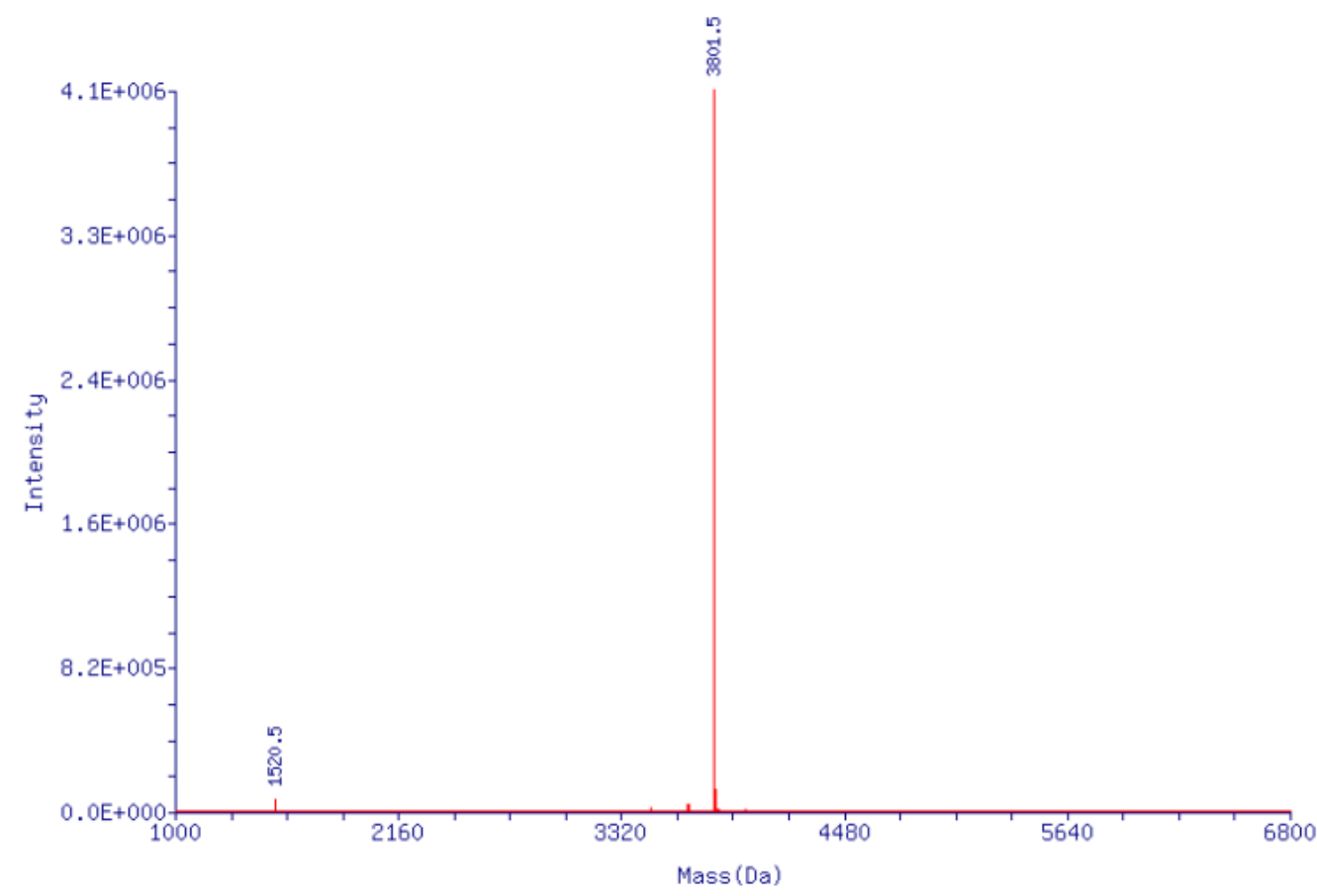

\section{Oligonucleotide 4}

Deconvoluted Mass Spectrum of A_2749865_3, RT = $0.375 \mathrm{~min}$ :

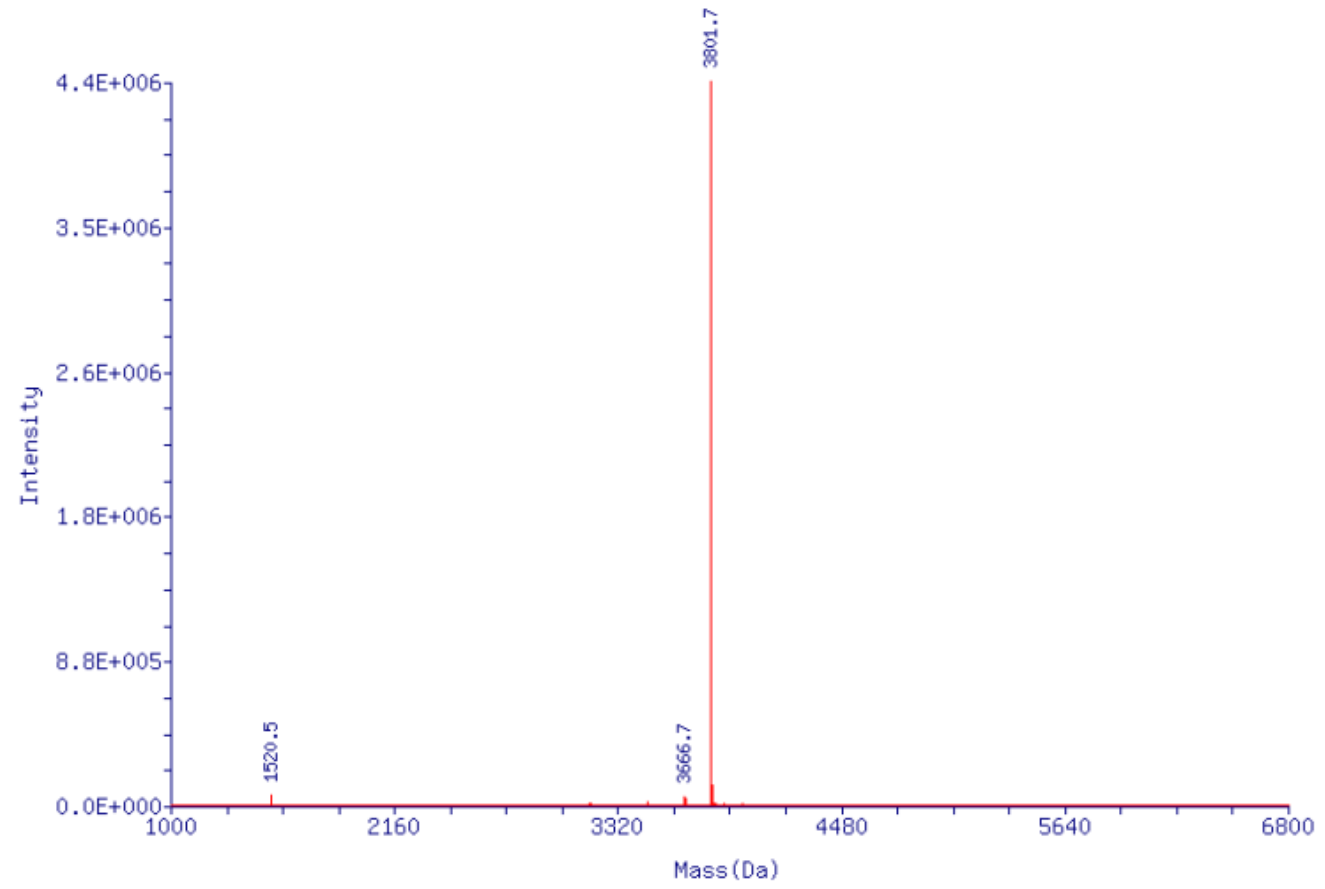




\section{Oligonucleotide 5}

Deconvoluted Mass Spectrum of A_2817182_1, RT = $0.376 \mathrm{~min}$ :

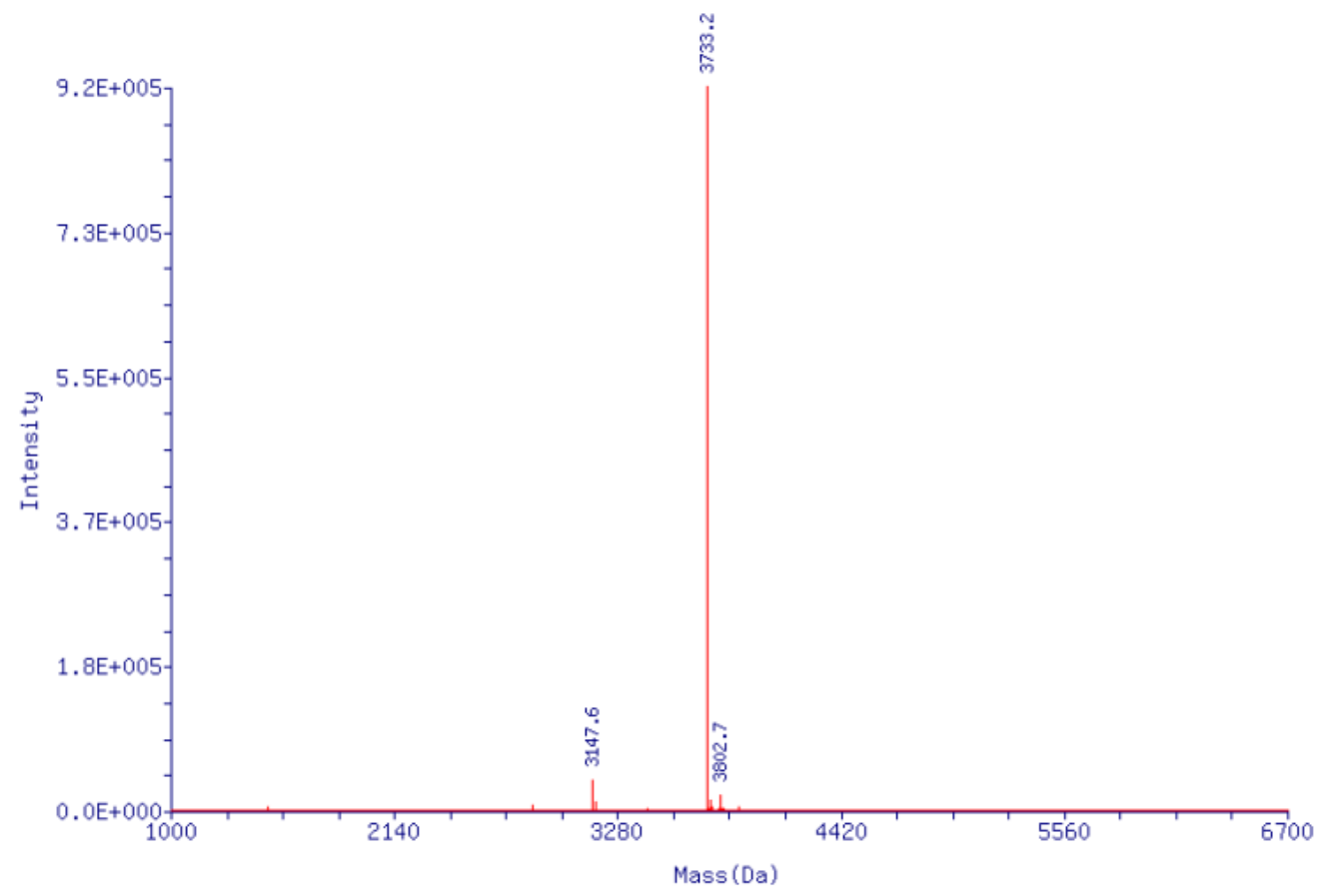

\section{Oligonucleotide 6}

Deconvoluted Mass Spectrum of A_2817183_1, RT = $0.360 \mathrm{~min}$ :

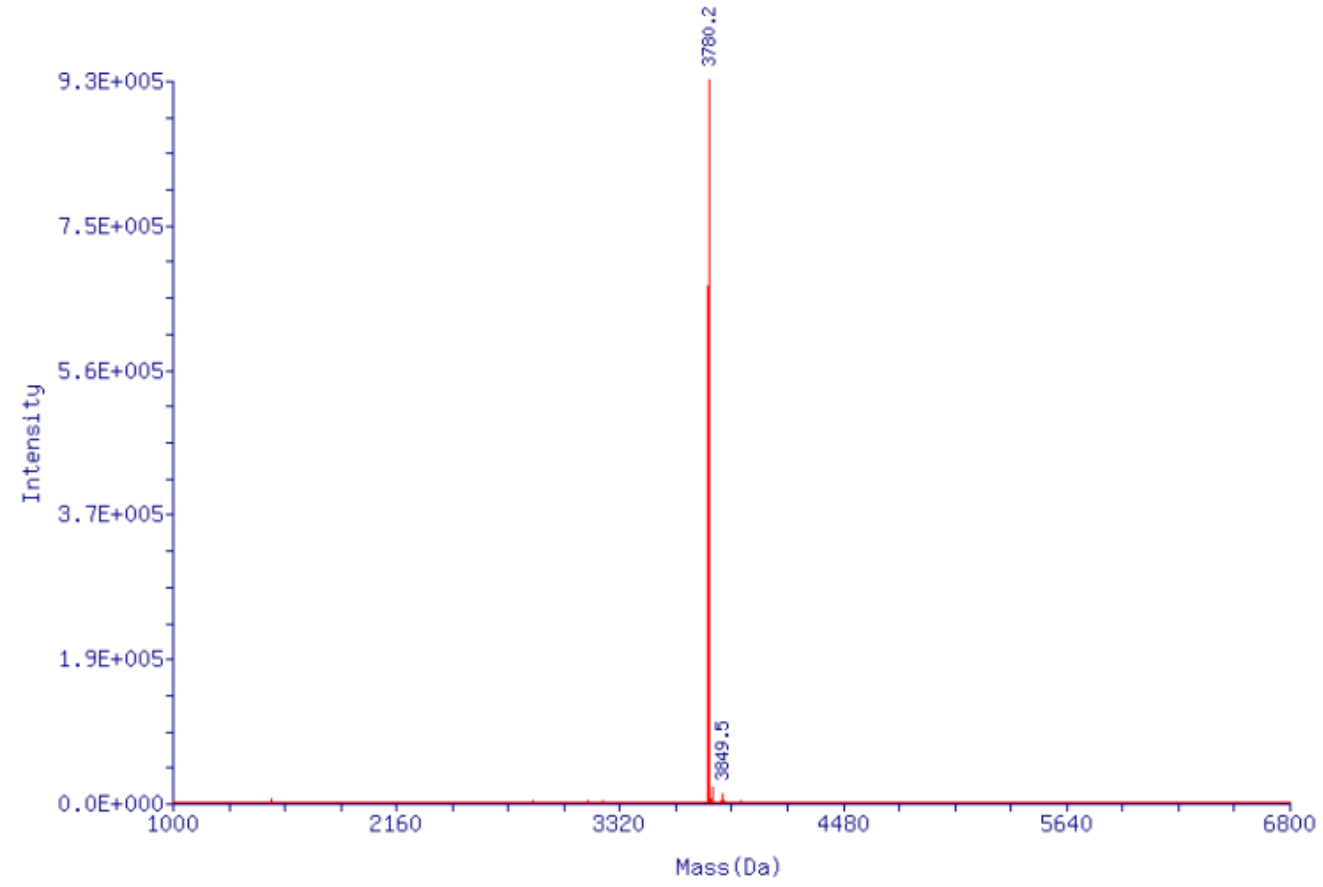




\section{Thermal melting studies}

UV melting curves were recorded using a Beckman Coulter DU® 800 Spectrophotometer. The concentration of duplex was $2 \mu \mathrm{M}$, and samples were prepared in PBS. Samples were annealed by heating to $95^{\circ} \mathrm{C}$ and then slowly cooled to $15^{\circ} \mathrm{C}$. Samples were then heated to $95{ }^{\circ} \mathrm{C}$ at a gradient of $1{ }^{\circ} \mathrm{C} / \mathrm{min}$, and the change in UV absorbance at $260 \mathrm{~nm}$ was recorded. The melting temperature was calculated from the first derivative of the melting curve.

\section{$T_{\mathrm{m}}$ profiles of the duplexes}

Unmodified RNA/RNA duplex

$5 '$-r(UACAGUCUAUGU)

3'-r(AUGUCAGAUACA)

Sample ID: 126502/126503

$\operatorname{Tm}=51 \cdot 3^{\circ} \mathrm{C}$

Reverse $\mathrm{Tm}=52.0{ }^{\circ} \mathrm{C}$

Solvent : 1X PBS

Salt Conc : 1.0000

DNA Length : 12

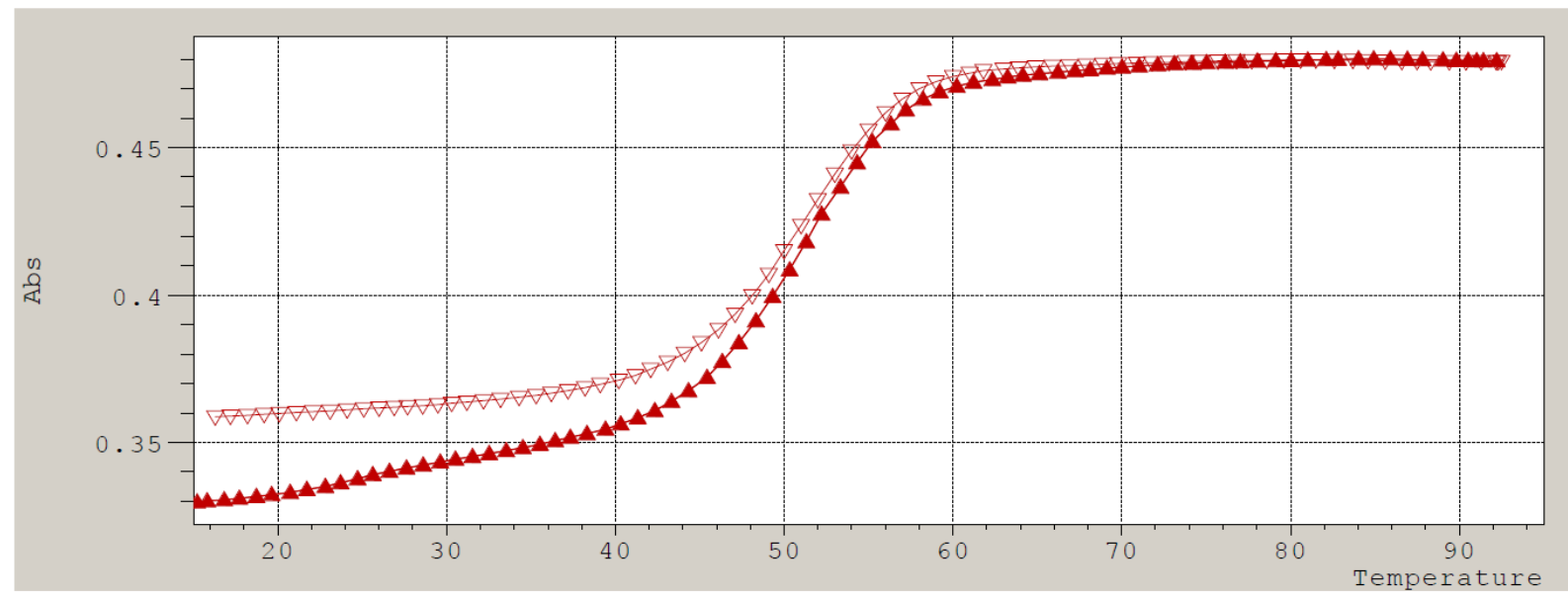




\section{Duplex1}

5'-r(UACAGUCUAUGU)

3'-r(AUGUCAGAUACA)

Sample ID: 2749866/126503

$\mathrm{Tm}=46.3^{\circ} \mathrm{C}$

Reverse $\mathrm{Tm}=46.1{ }^{\circ} \mathrm{C}$

Solvent : $1 \mathrm{X}$ PBS

Salt Conc : 1.0000

DNA Length : 12

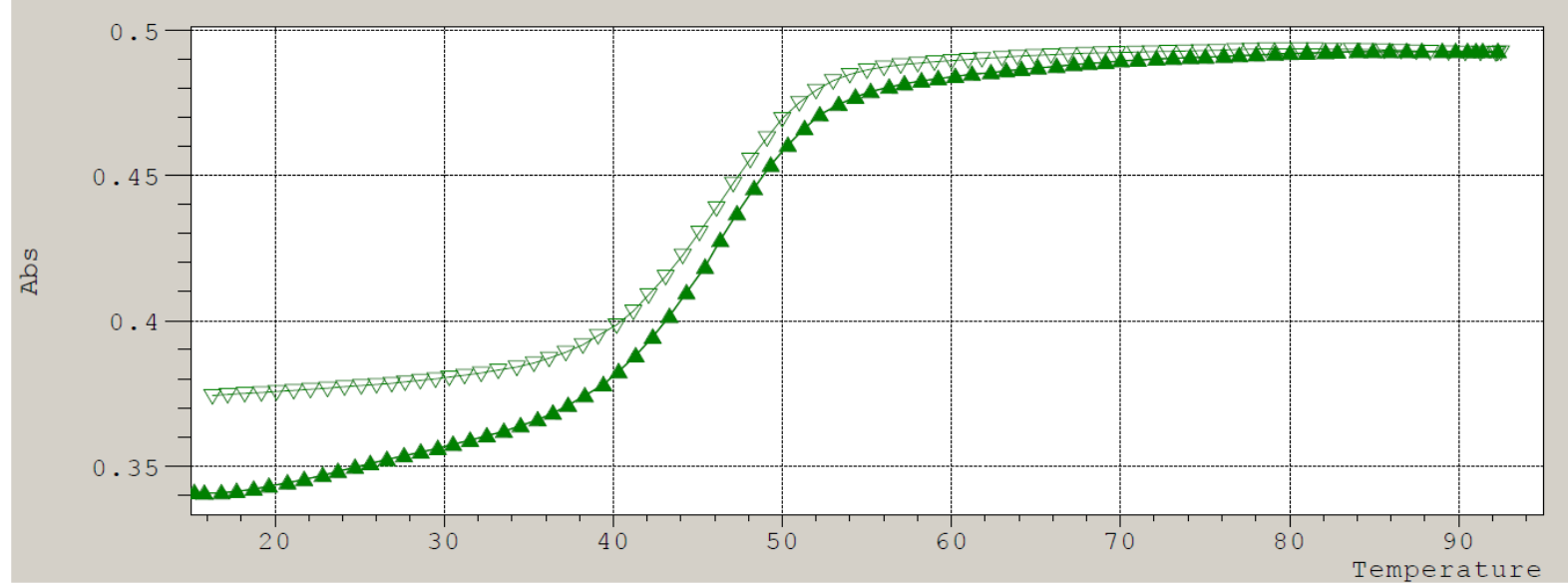

\section{Duplex 2}

5'-r(UACAGUCUAUGU)

3'-r(AUGUCAGAUACA)

Sample ID: 274964/126503

$\mathrm{Tm}=46.3^{\circ} \mathrm{C}$

Reverse $\mathrm{Tm}=46.1{ }^{\circ} \mathrm{C}$

Solvent : 1X PBS

Salt Conc : 1.0000

DNA Length : 12

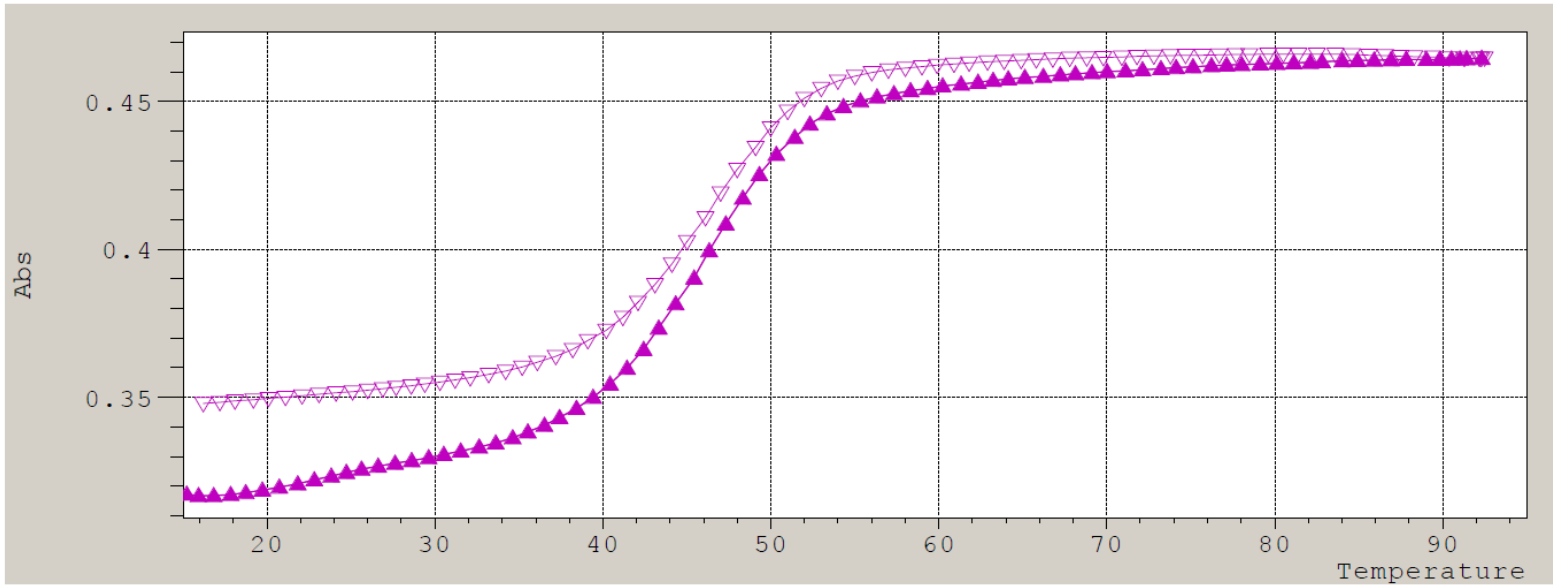


Duplex 3

5'-r(UACAGUCUAUGU)

3'-r(AUGUCAGAUACA)

Sample ID: 126502/2749863

$\mathrm{Tm}=47.4{ }^{\circ} \mathrm{C}$

Reverse $\mathrm{Tm}=48.0{ }^{\circ} \mathrm{C}$

Solvent : 1X PBS

Salt Conc : 1.0000

DNA Length : 12

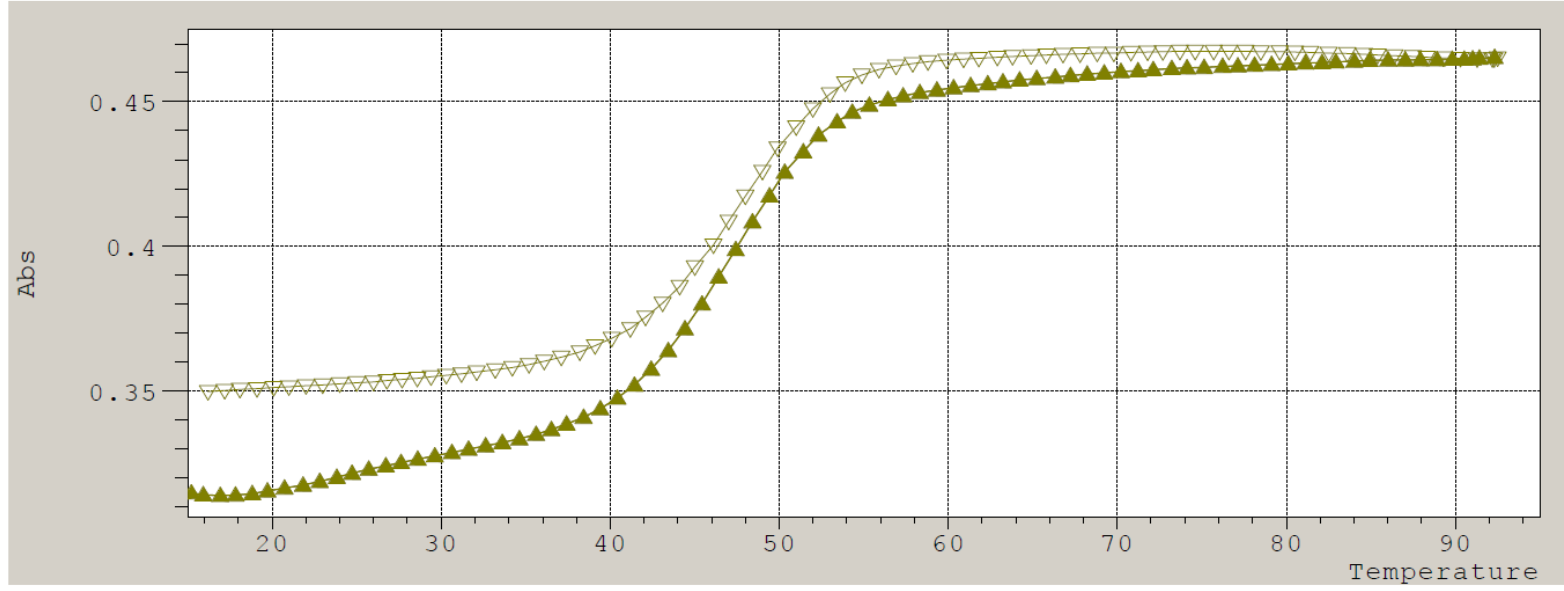

Duplex 4

5'-r(UACAGUCUAUGU)

3'-r(AUGUCAGAUACA)

Sample ID: 126502/2749865

$\mathrm{Tm}=46.4^{\circ} \mathrm{C}$

Reverse $\mathrm{Tm}=47.0^{\circ} \mathrm{C}$

Solvent : 1X PBS

Salt Conc : 1.0000

DNA Length : 12

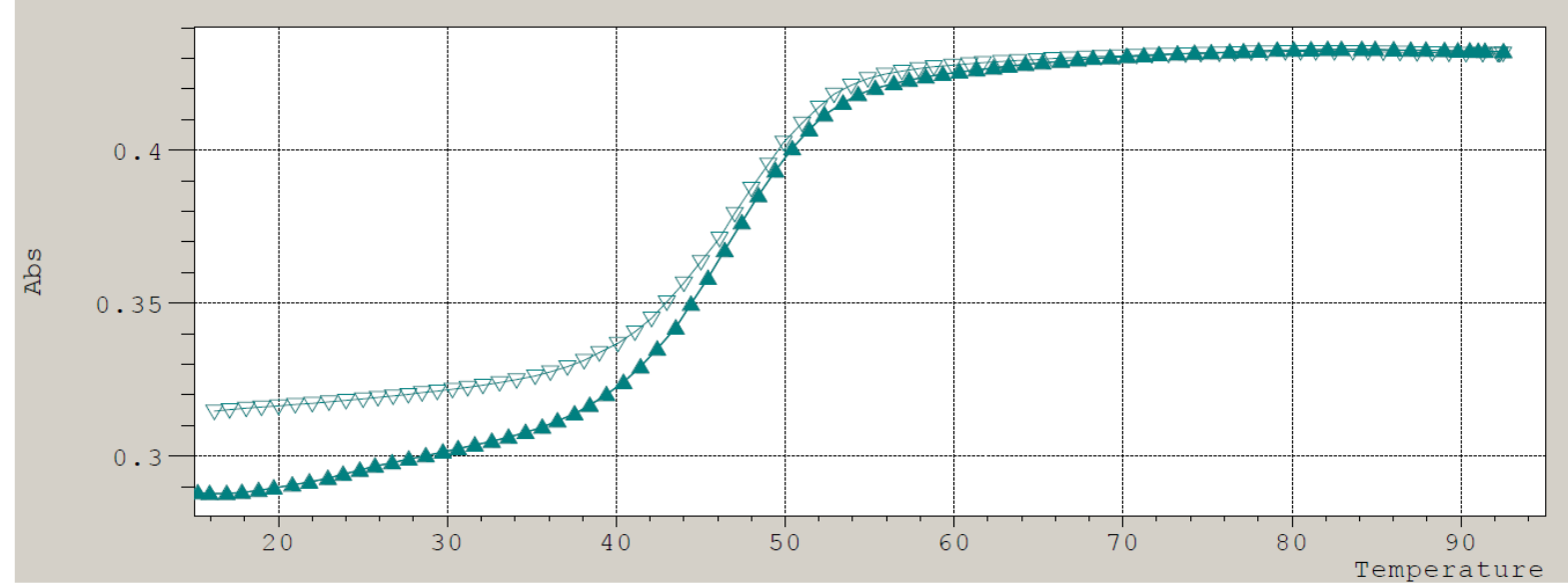


Duplex 5

5'-r(UACAGUCUAUGU)

3'-r(AUGUCAGAUACA)

Sample ID: 2817182/126503

Solvent : $1 \mathrm{X}$ PBS

Salt Conc : 1.0000

DNA Length : 12

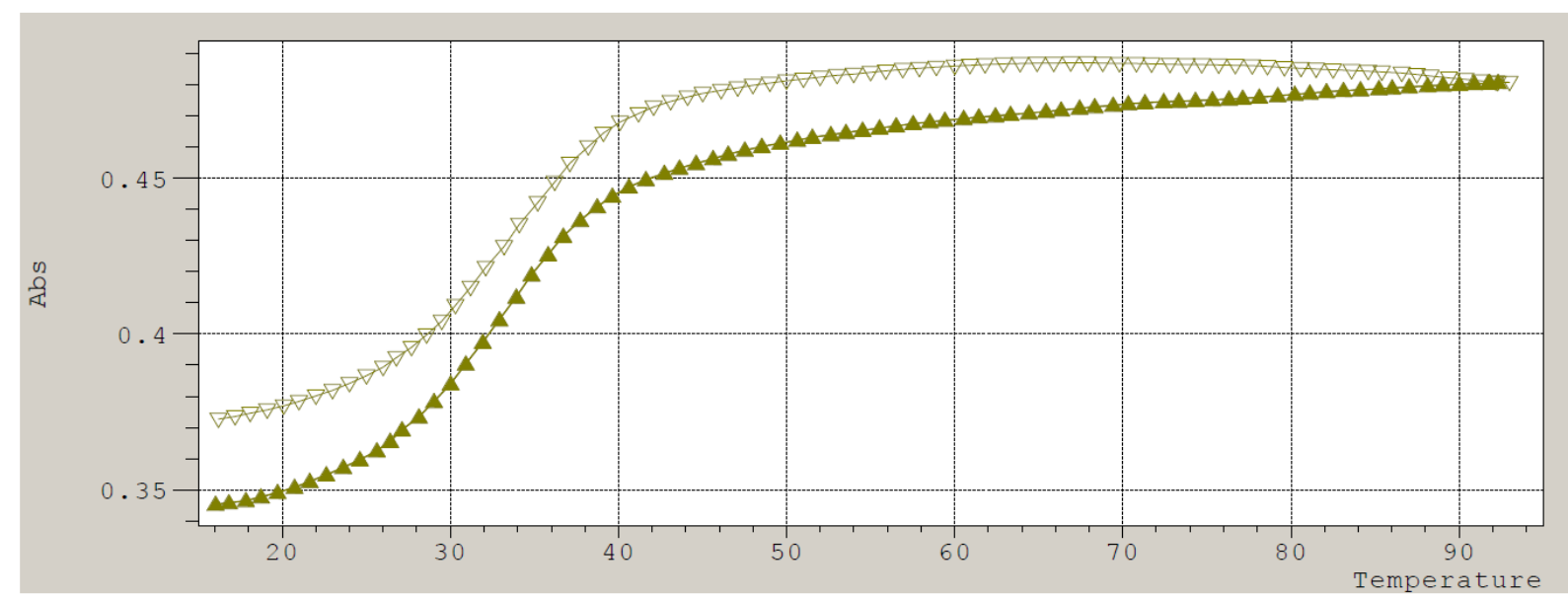

Duplex 6

5'-r(UACAGUCUAUGU)

3'-r(AUGUCAGAUACA)

Sample ID: $2817182 / 2817183$

Solvent : $1 \mathrm{X}$ PBS

$\operatorname{Tm}=32.9^{\circ} \mathrm{C}$

Reverse $\mathrm{Tm}=34.1{ }^{\circ} \mathrm{C}$

Salt Conc : 1.0000

DNA Length : 12

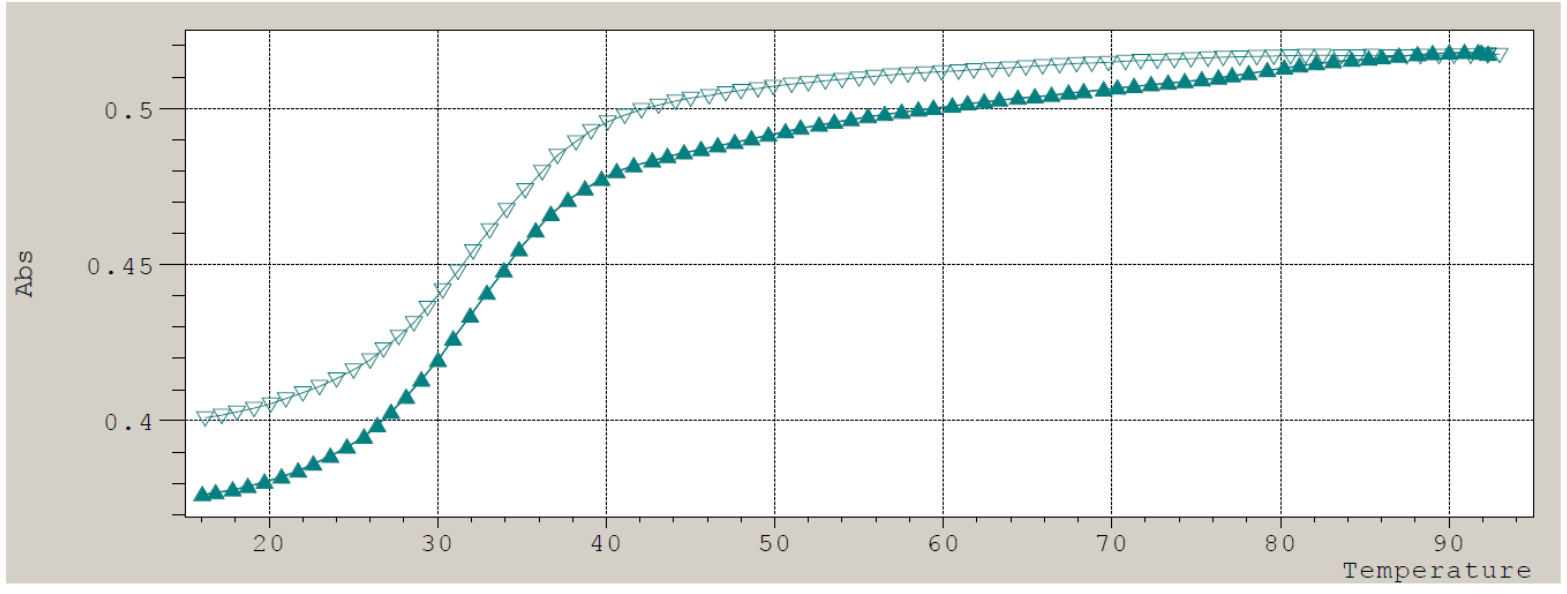


Duplex 7

5'-r(UACAGUCUAUGU)

3'-r(AUGUCAGAUACA)

Sample ID: 2817182/2817183

$\operatorname{Tm}=19.7{ }^{\circ} \mathrm{C}$

Reverse $\mathrm{Tm}=47.1{ }^{\circ} \mathrm{C}$

Solvent : 1X PBS

Salt Conc : 1.0000

DNA Length : 12

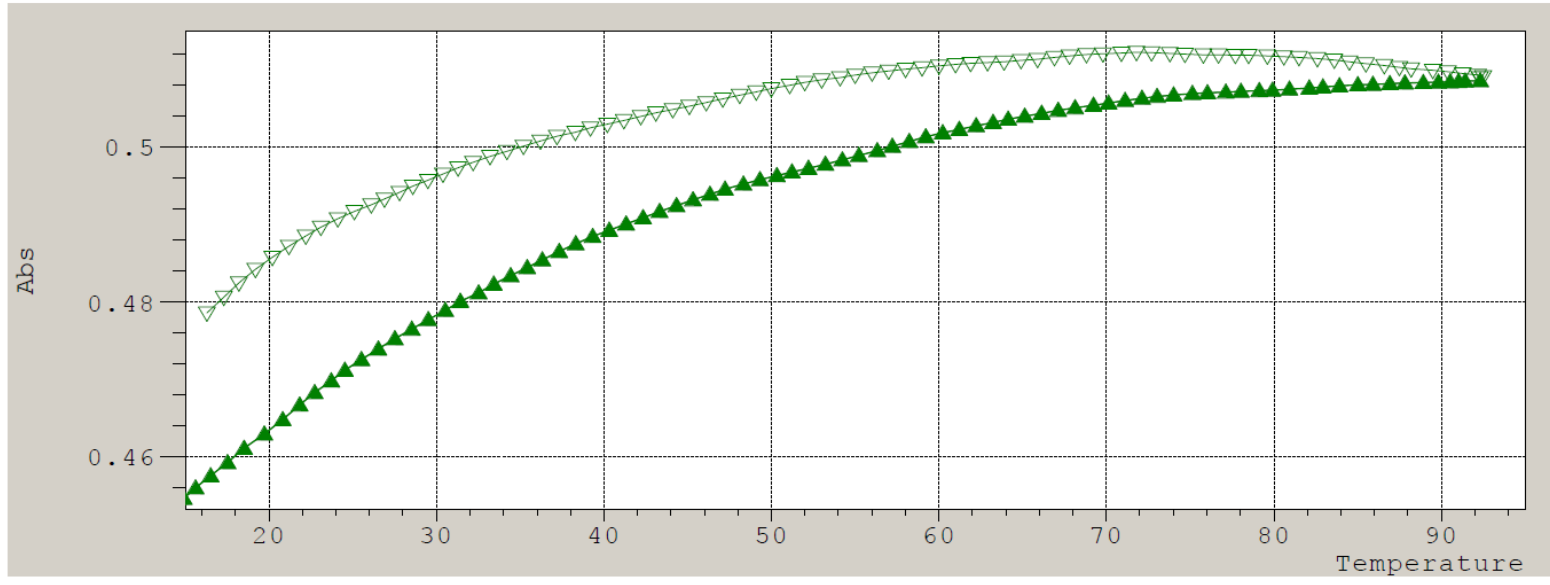


Unmodified RNA/DNA 1

5'-r(UACAGUCUAUGU)

3'-d(ATGTCAGATACA)
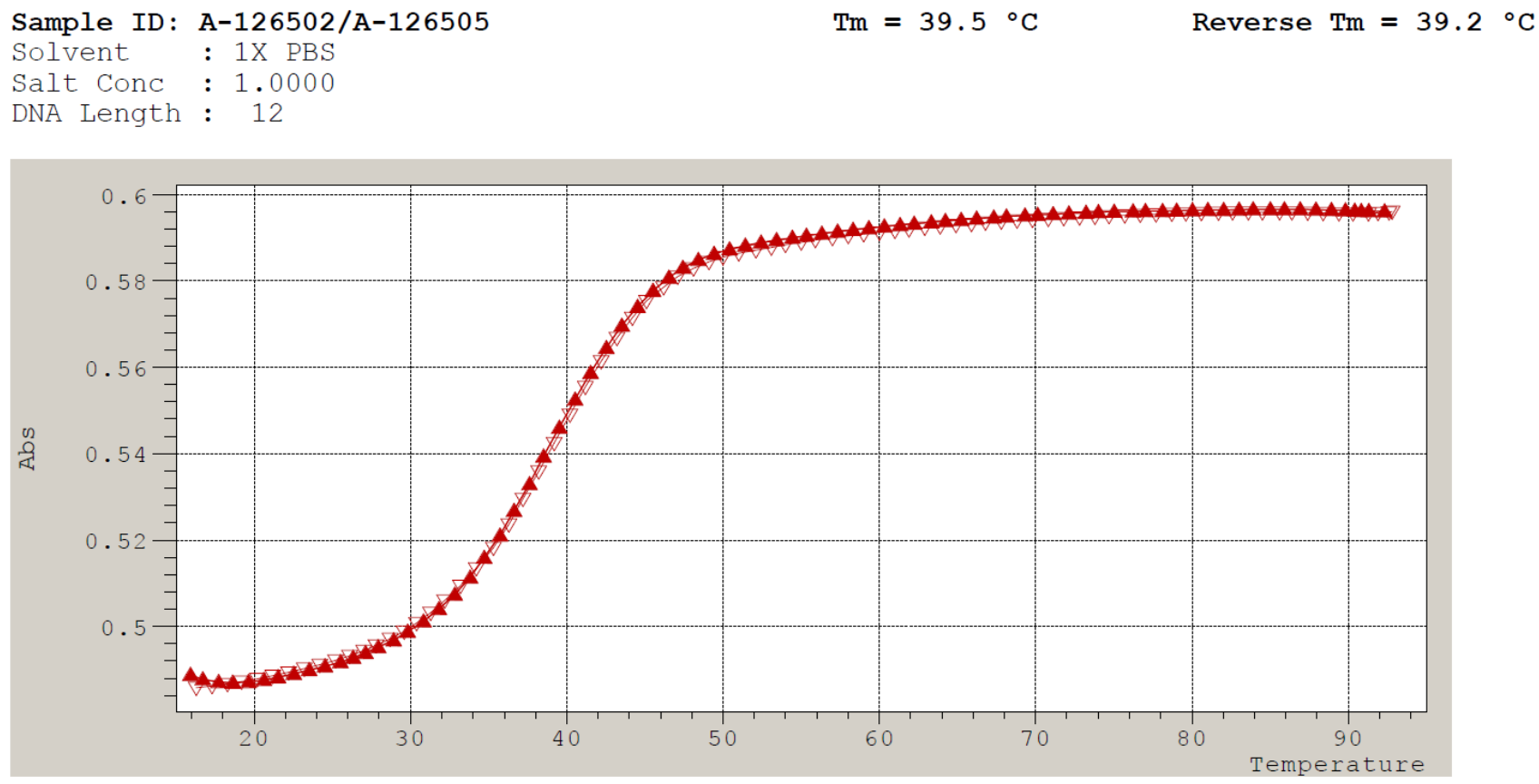

Duplex 8

5'-r(UACAGUCUAUGU) 3'-d(ATGTCAGATACA)

Sample ID: A-2749866/A-126505

Solvent

: $1 \mathrm{X}$ PBS

Salt Conc : 1.0000

DNA Length : 12

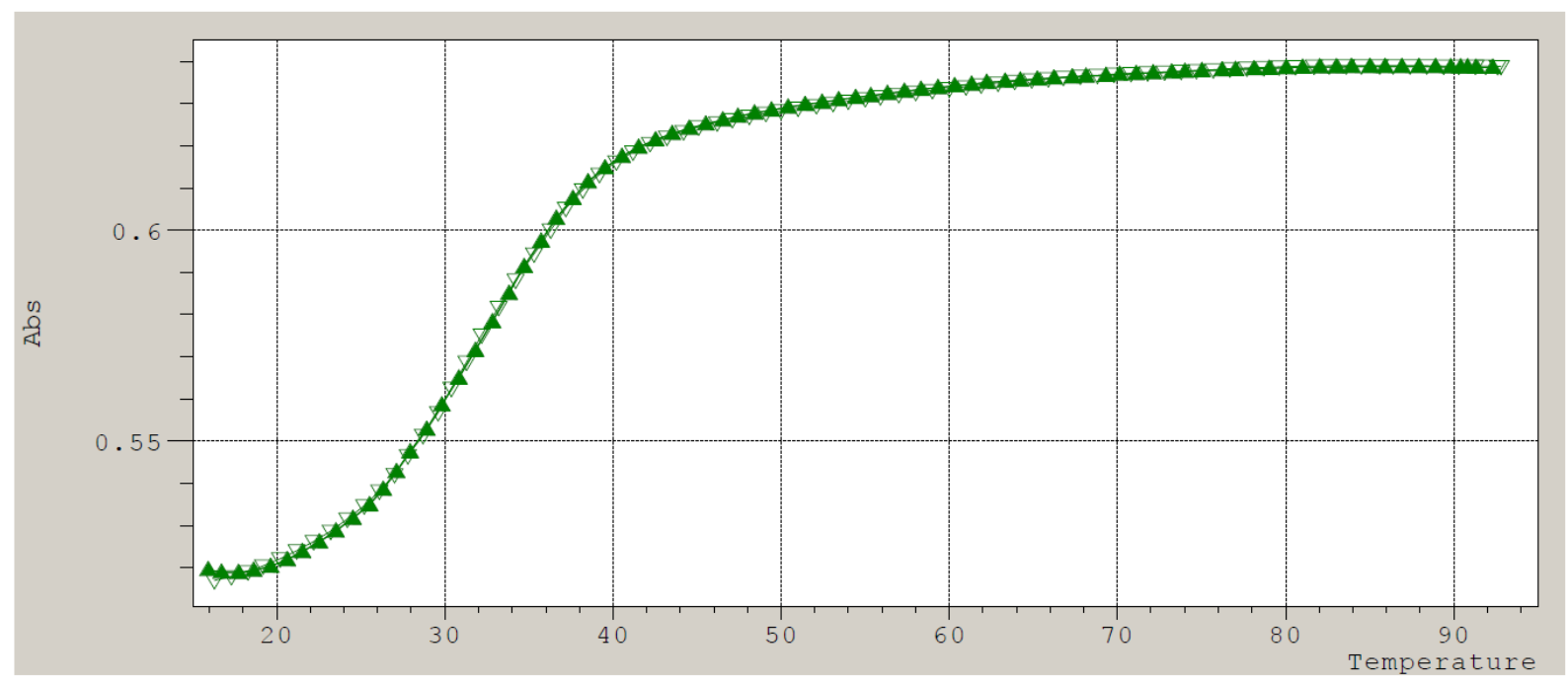


Duplex 9

5'-r(UACAGUCUAUGU)

3'-d(ATGTCAGATACA)

Sample ID: A-2749864/A-126505

$\mathrm{Tm}=30.8^{\circ} \mathrm{C}$

Reverse $\mathrm{Tm}=32.1{ }^{\circ} \mathrm{C}$

Solvent : 1X PBS

Salt Conc : 1.0000

DNA Length : 12

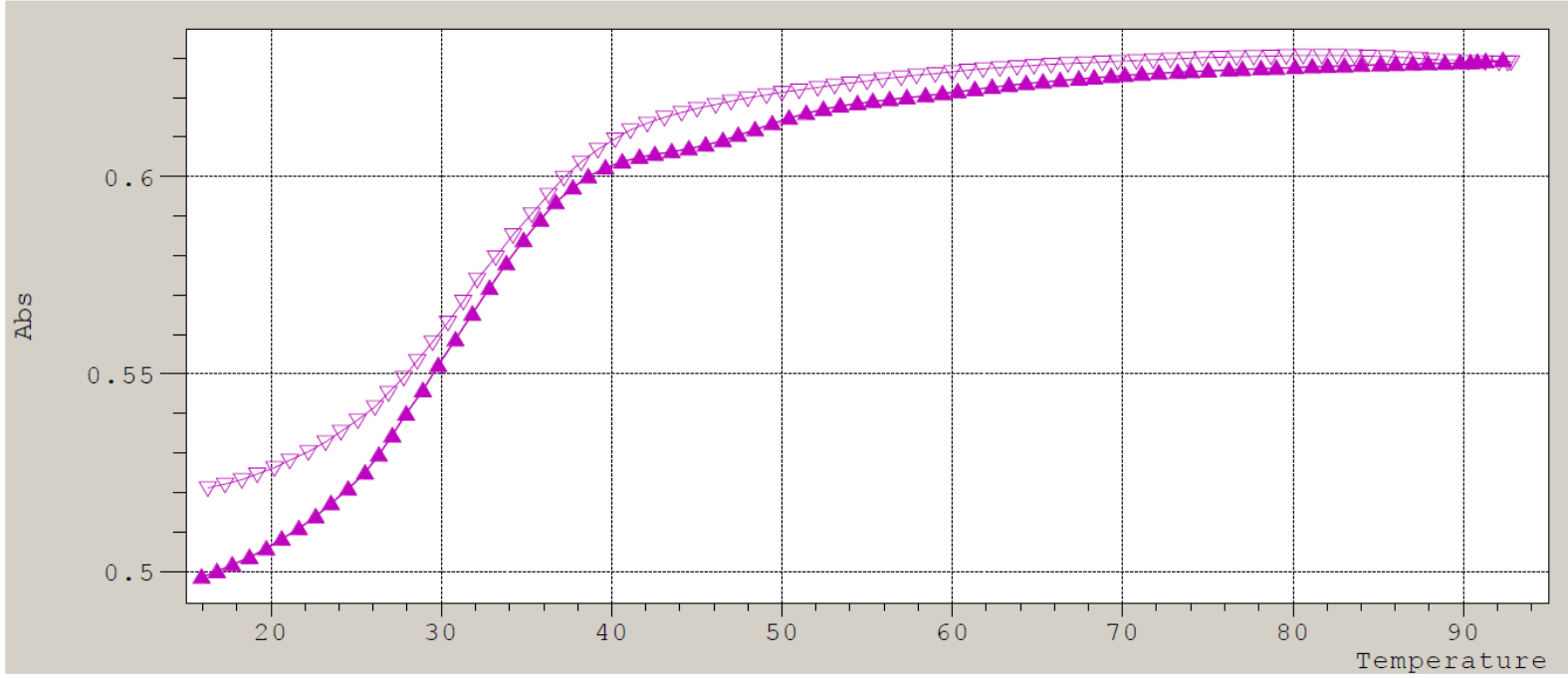

Duplex 12

5'-r(UACAGUCUAUGU)

3'-d(ATGTCAGATACA)

Sample ID: A-2817182/A-126505

$\mathrm{Tm}=57.5^{\circ} \mathrm{C}$

Reverse $\mathrm{Tm}=55.9{ }^{\circ} \mathrm{C}$

Solvent : $1 \mathrm{X}$ PBS

Salt Conc : 1.0000

DNA Length : 12

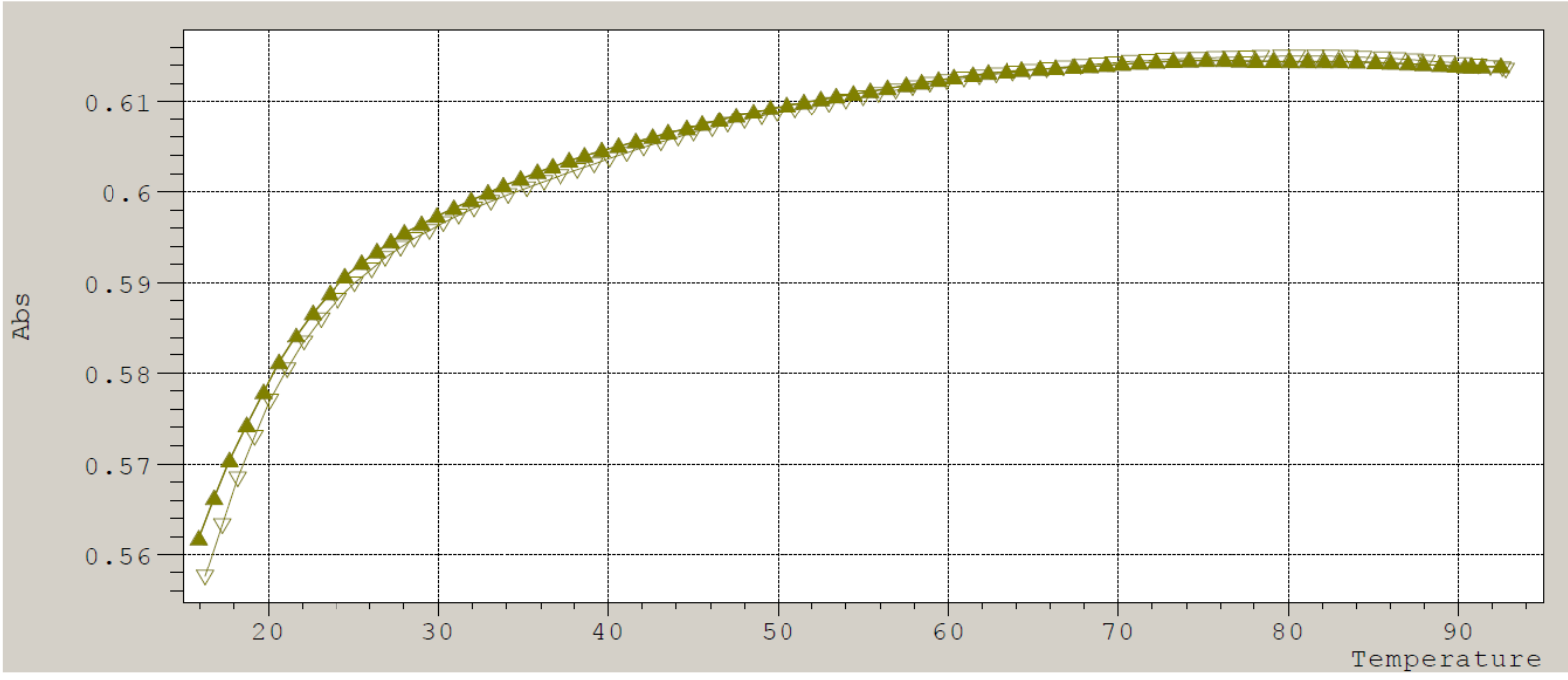


Unmodified RNA/DNA 2

5'-d(TACAGTCTATGT)

3'-r(AUGUCAGAUACA)

Sample ID: A-126504/A-126503

$\operatorname{Tm}=40.6^{\circ} \mathrm{C}$

Reverse $\mathrm{Tm}=41.2{ }^{\circ} \mathrm{C}$

solvent : 1X PBS

Salt conc : 1.0000

DNA Length : 12

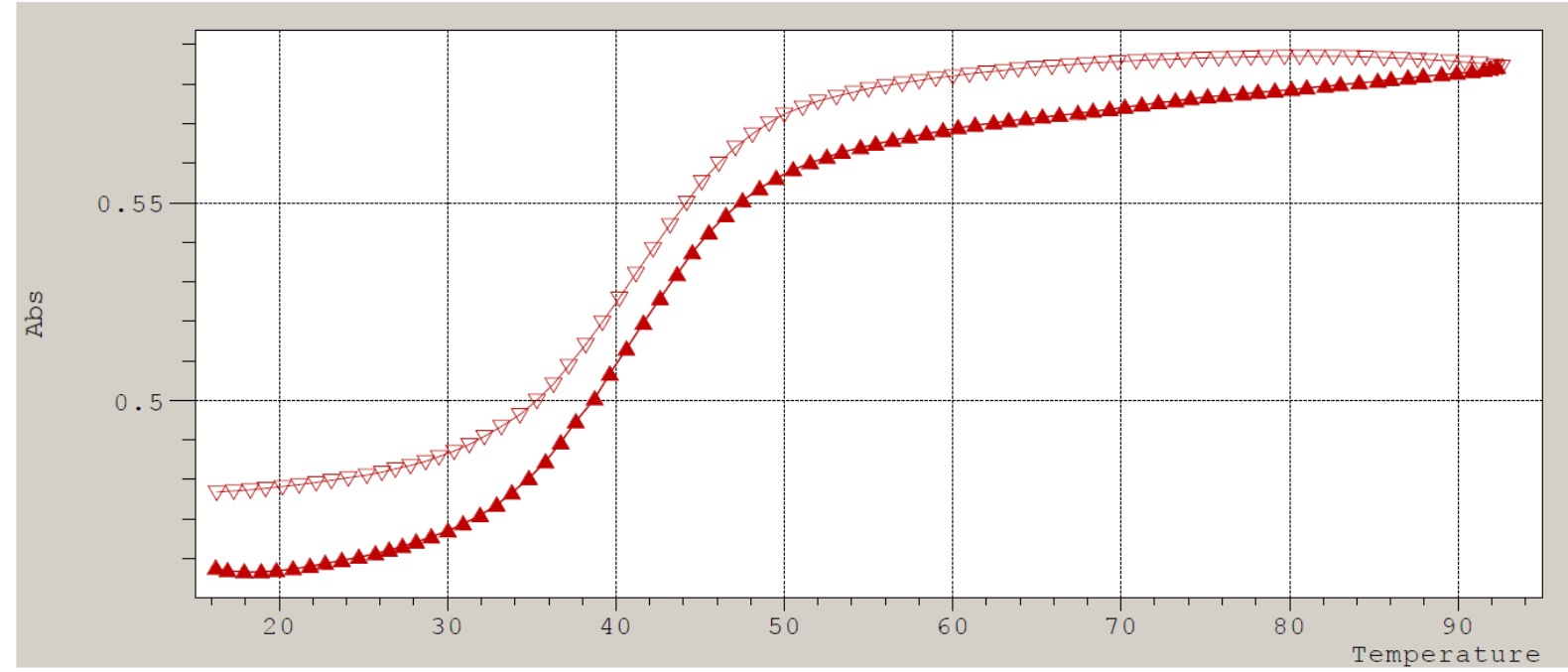

Duplex 10

5'-d(TACAGTCTATGT)

3'-r(AUGUCAGAUACA)

Sample ID: A-126504/A-2749863

$\operatorname{Tm}=36.7^{\circ} \mathrm{C}$

Reverse $\mathrm{Tm}=36.3{ }^{\circ} \mathrm{C}$

Solvent : 1X PBS

Salt Conc : 1.0000

DNA Length : 12

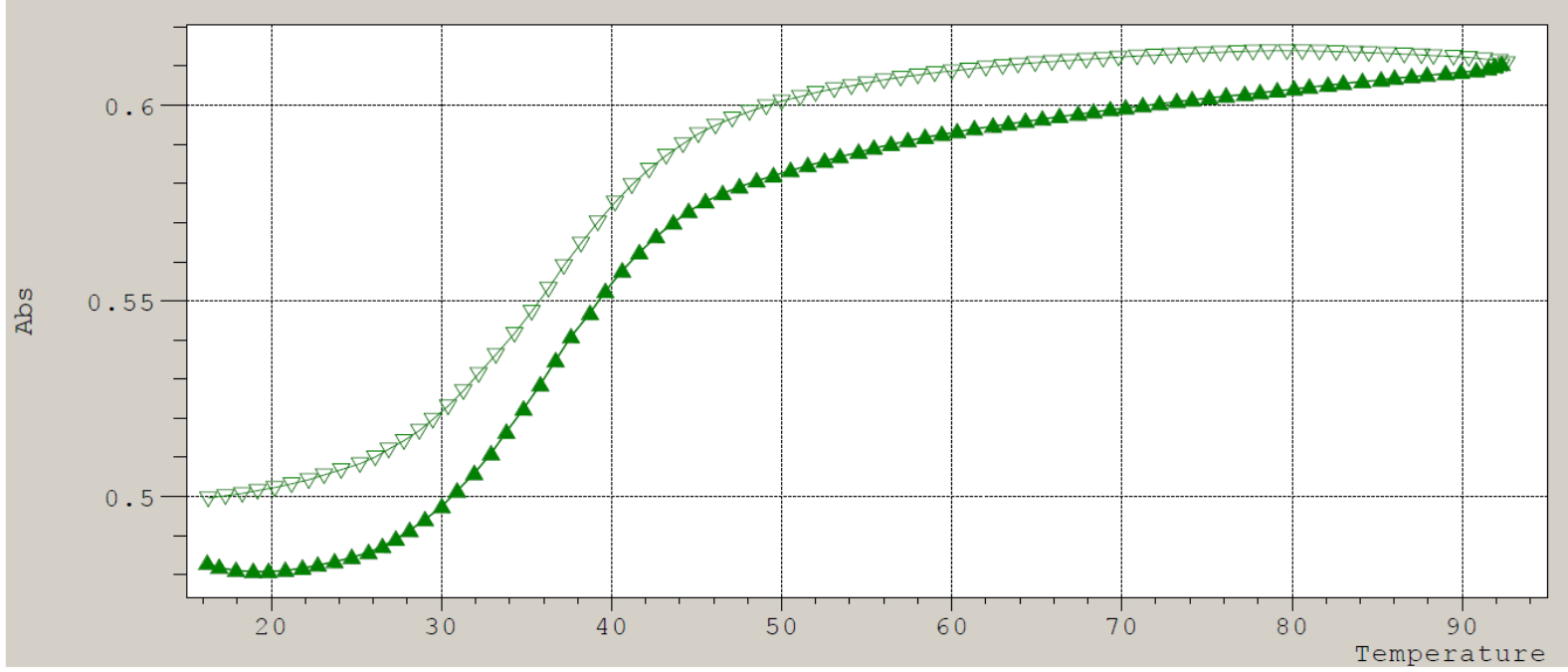


Duplex 11

5'-d(TACAGTCTATGT)

3'-r(AUGUCAGAUACA)

Sample ID: A-126504/A-2749865

Solvent : 1X PBS

Salt Conc : 1.0000

DNA Length : 12

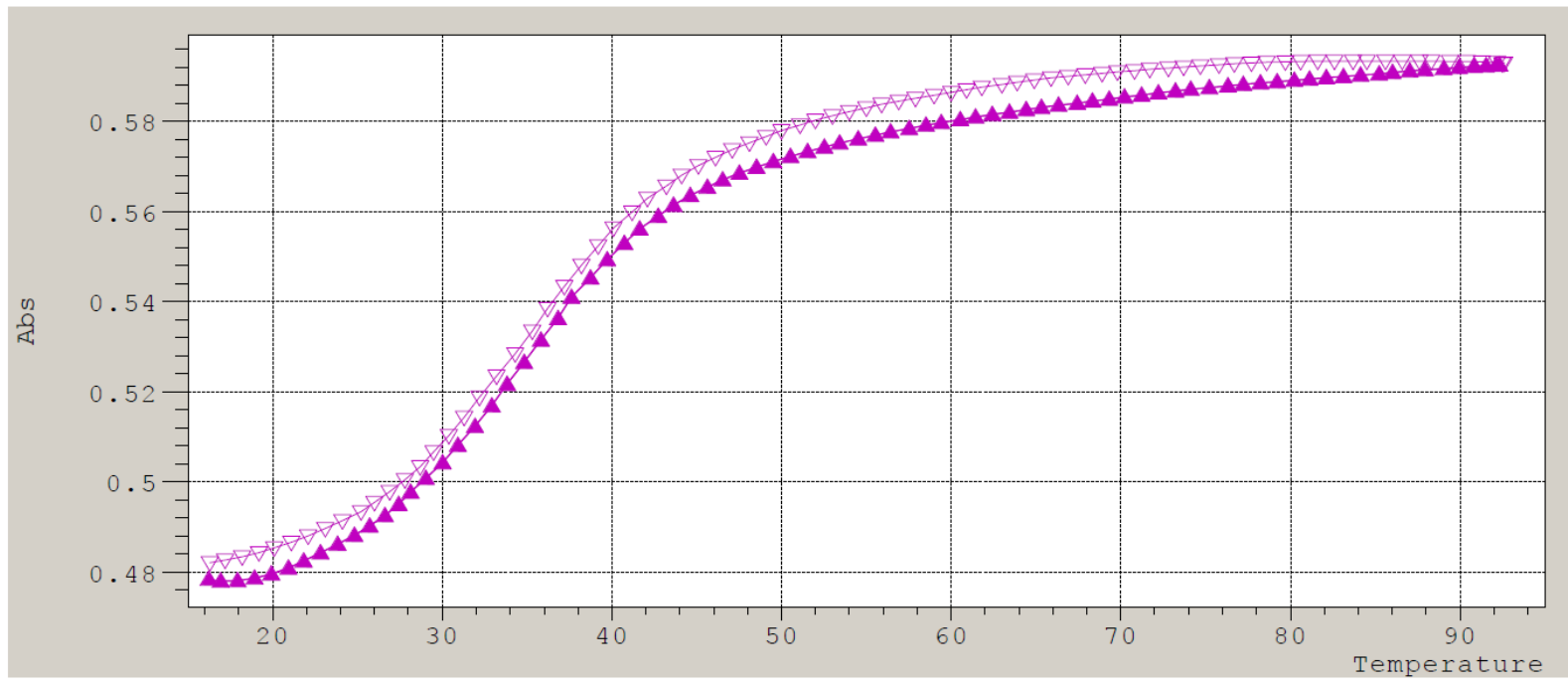

Duplex 13

5'-d(TACAGTCTATGT)

3'-r(AUGUCAGAUACA)

Sample ID: A-126504/A-2817183

Solvent : $1 \mathrm{X}$ PBS

Salt Conc : 1.0000

DNA Length : 12

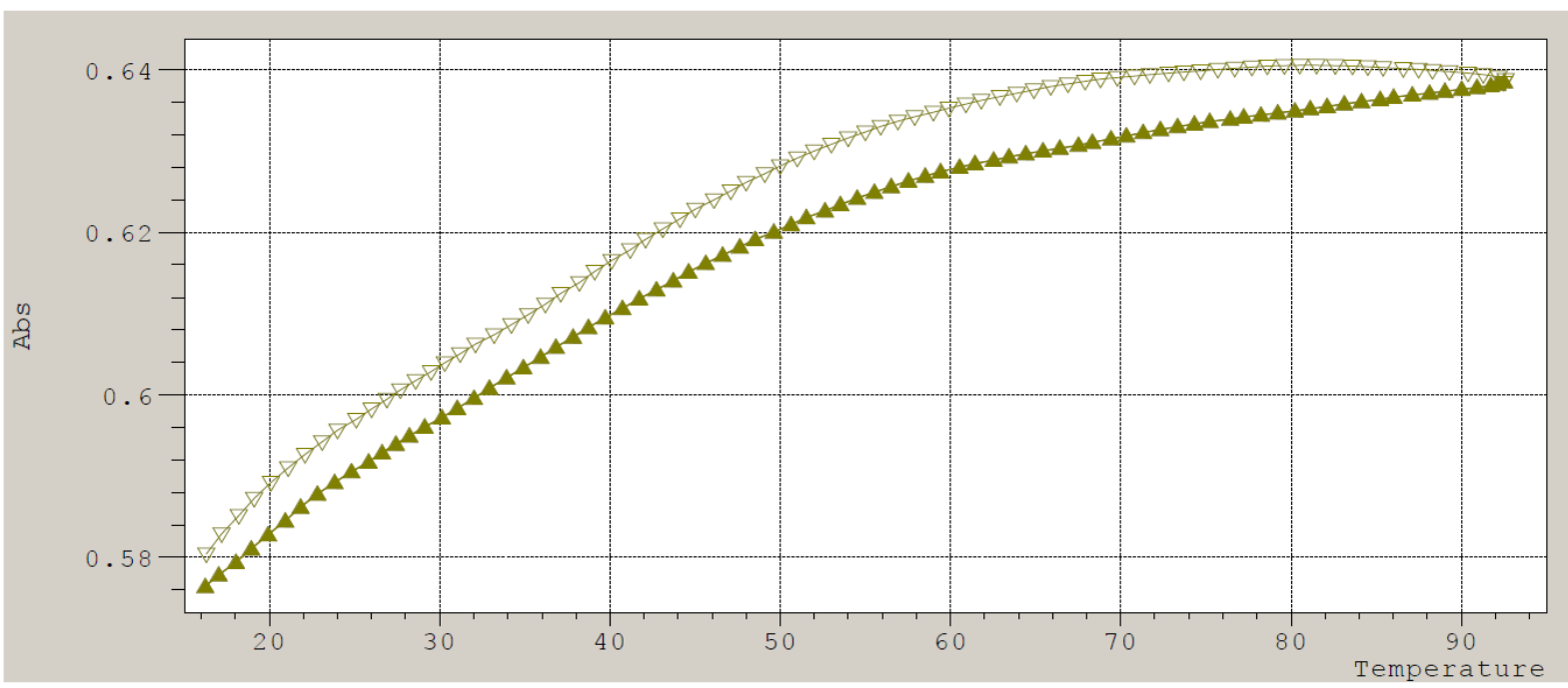




\section{Circular dichroism (CD) spectroscopy}

The CD spectra were obtained on a Jasco J-815 spectropolarimeter equipped with a Julaba F25 circulating bath. The sample was allowed to equilibrate at $15^{\circ} \mathrm{C}$ in $\mathrm{PBS}$ at a final concentration of $2.0 \mu \mathrm{M}$ duplex. The spectrum was an average of 5 scans. Spectra were collected at a rate of 100 $\mathrm{nm} / \mathrm{min}$, with a bandwidth of $1 \mathrm{~nm}$ and sampling wavelength of $0.2 \mathrm{~nm}$ using fused quartz cells (Starna 29-Q-10). The CD spectra were recorded from 350 to $200 \mathrm{~nm}$ at $15^{\circ} \mathrm{C}$. The molar ellipticity was calculated from the equation $[\theta]=\theta / 10 \mathrm{Cl}$, where $\theta$ is the ellipticity (mdeg), $\mathrm{C}$ is the molar concentration of oligonucleotides $(\mathrm{M})$, and 1 is the path length of the cell $(\mathrm{cm})$. The data were processed on a PC computer using Windows ${ }^{\mathrm{TM}}$ based software supplied by the manufacturer (JASCO, Inc.) and transferred into Microsoft Excel ${ }^{\mathrm{TM}}$ for presentation.

A

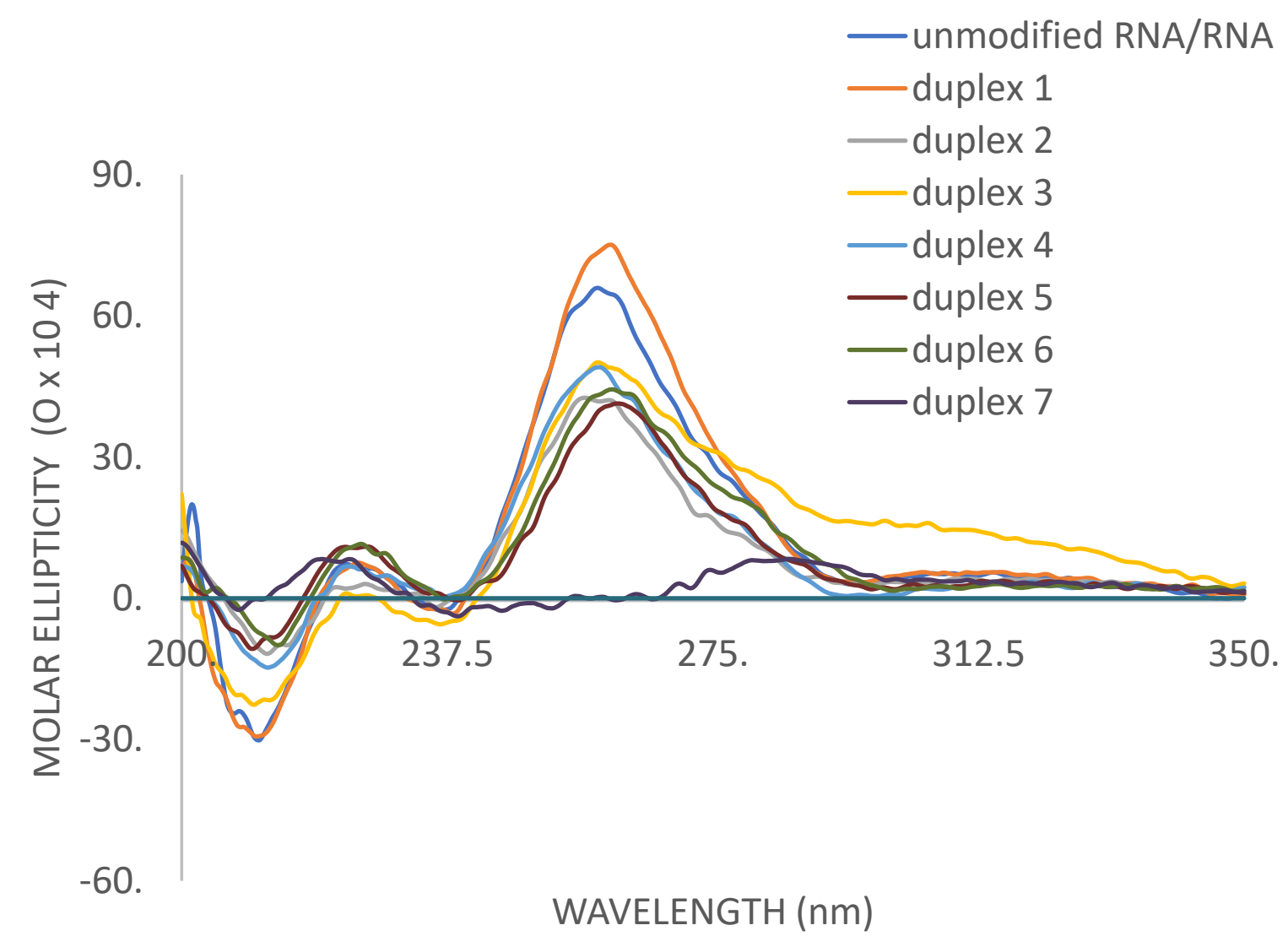




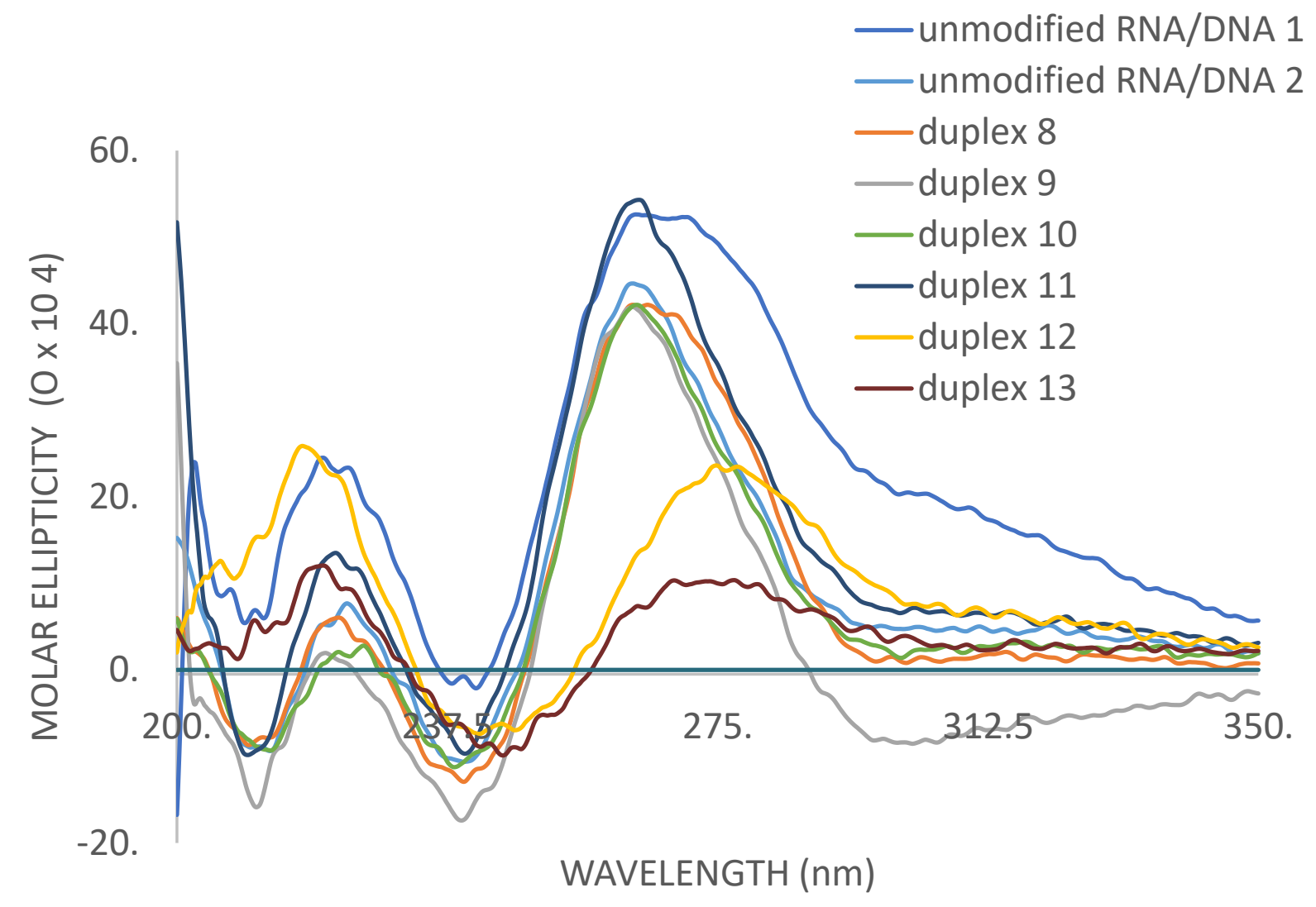

Figure S1. CD spectra of unmodified RNA duplex and (A) RNA duplexes 1-7 and (B) RNA/DNA duplexes 8-13. Sequences of unmodified RNA/DNA duplexes 1 and 2 are 5'r(UACAGUCUAUGU)-3': $3^{\prime}$-d(ATGTCAGATACA)-5' and 5'-d(TACAGTCTATGT)-3':3'r(AUGUCAGAUACA)-5', respectively. Spectra are the averages of 5 scans and were recorded at $15{ }^{\circ} \mathrm{C}$. 


\section{References}

(1) Weising, S.; Dekiert, P.; Schols, D.; Neyts, J.; Meier, C., Synthesis of Enantiomerically Pure 1',2'-cis-dideoxy, -dideoxydi-dehydro, -ribo and -deoxy Carbocyclic Nucleoside Analogues. Synthesis 2018, 50, 2266-2280.

(2) Rassu, G.; Auzzas, L.; Pinna, L.; Zambrano, V.; Zanardi, F.; Battistini, L.; Marzocchi, L.; Acquotti, D.; Casiraghi, G., Variable Strategy toward Carbasugars and Relatives. 4.1 Viable Access to (4a-Carbapentofuranosyl)amines, (5a-Carbahexopyranosyl)amines, and Amino Acids Thereof. J. Org. Chem. 2002, 67, 5338-5342.

(3) Singh, U. S.; Mishra, R. C.; Shankar, R.; Chu, C. K., Stereoselective Synthesis of 2'-Fluoro6'-methylene Carbocyclic Adenosine via Vince Lactam. J. Org. Chem. 2014, 79, 3917-3923.

(4) Krause, L.; Herbst-Irmer, R.; Sheldrick, G. M.; Stalke, D., Comparison of silver and molybdenum microfocus X-ray sources for single-crystal structure determination. J. Appl. Cryst. 2015, 48, 3-10.

(5) Sheldrick, G., SHELXT - Integrated space-group and crystal-structure determination. Act. Cryst. A 2015, 71, 3-8.

(6) Sheldrick, G., Crystal structure refinement with SHELXL. Act. Cryst. C 2015, 71, 3-8.

(7) Parsons, S.; Flack, H. D.; Wagner, T., Use of intensity quotients and differences in absolute structure refinement. Act. Cryst. B 2013, 69, 249-259.

(8) Hubschle, C. B.; Sheldrick, G. M.; Dittrich, B., ShelXle: a Qt graphical user interface for SHELXL. J. Appl. Cryst. 2011, 44, 1281-1284. 